\title{
Detection of developmental toxicity using differentiating embryonic stem cells : a transcriptomic approach
}

Citation for published version (APA):

van Dartel, D. A. (2011). Detection of developmental toxicity using differentiating embryonic stem cells : a transcriptomic approach. [Doctoral Thesis, Maastricht University]. Universiteit Maastricht. https://doi.org/10.26481/dis.20111014dd

Document status and date:

Published: 01/01/2011

DOI:

10.26481/dis.20111014dd

Document Version:

Publisher's PDF, also known as Version of record

\section{Please check the document version of this publication:}

- A submitted manuscript is the version of the article upon submission and before peer-review. There can be important differences between the submitted version and the official published version of record.

People interested in the research are advised to contact the author for the final version of the publication, or visit the DOI to the publisher's website.

- The final author version and the galley proof are versions of the publication after peer review.

- The final published version features the final layout of the paper including the volume, issue and page numbers.

Link to publication

\footnotetext{
General rights rights.

- You may freely distribute the URL identifying the publication in the public portal. please follow below link for the End User Agreement:

www.umlib.nl/taverne-license

Take down policy

If you believe that this document breaches copyright please contact us at:

repository@maastrichtuniversity.nl

providing details and we will investigate your claim.
}

Copyright and moral rights for the publications made accessible in the public portal are retained by the authors and/or other copyright owners and it is a condition of accessing publications that users recognise and abide by the legal requirements associated with these

- Users may download and print one copy of any publication from the public portal for the purpose of private study or research.

- You may not further distribute the material or use it for any profit-making activity or commercial gain

If the publication is distributed under the terms of Article $25 \mathrm{fa}$ of the Dutch Copyright Act, indicated by the "Taverne" license above, 


\section{Detection of developmental toxicity}

using differentiating embryonic stem cells

A transcriptomic approach

Dorien van Dartel 
ISBN: 978-94-6169-045-6

Layout and printing: Optima Grafische Communicatie, Rotterdam, The Netherlands 


\title{
Detection of developmental toxicity using differentiating embryonic stem cells A transcriptomic approach
}

\author{
Proefschrift \\ ter verkrijging van de graad van doctor aan de Universiteit Maastricht, \\ op gezag van de Rector Magnificus, prof. mr. G.P.M.F. Mols \\ volgens het besluit van het College van Decanen, \\ in het openbaar te verdedigen \\ op vrijdag 14 oktober 2011 om 14.00 uur \\ door \\ Dorien Anna Maria van Dartel \\ geboren te Schijndel op 3 februari 1983
}




\section{Promotores}

Prof. dr. J.C.S. Kleinjans

Prof. dr. A.H. Piersma (Universiteit Utrecht, RIVM)

\section{Copromotor}

Dr. J.L.A. Pennings (RIVM)

\section{Beoordelingscommissie}

Prof. dr. J.P.M. Geraedts (voorzitter)

Prof. dr. M. van den Berg (Universiteit Utrecht)

Prof. dr. W.H. Lamers

Prof. dr. H. van Loveren

Prof. dr. C.L. Mummery (Leids Universitair Medisch Centrum)

This research was financially supported by the Dutch Technology Foundation STW (MFA6809).

Financial support of the printing costs of this thesis by the National Institute for Public Health and the Environment (RIVM), the foundation Stimuleringsfonds Alternatieven voor Proefdieren, the Dierenbescherming, and the foundation PETA US is gratefully acknowledged. 
Abbreviations

Chapter 1 General introduction 11

Chapter 2 Disentangling cellular proliferation and differentiation in the Embryonic Stem cell Test, and its impact on the experimental protocol

Chapter 3 Early gene expression changes during embryonic stem cell differentiation into cardiomyocytes and their modulation by monobutyl phthalate

Chapter 4 Transcriptomics-based identification of developmental toxicants 65 through their interference with cardiomyocyte differentiation of embryonic stem cells

Chapter 5 Monitoring developmental toxicity in the Embryonic Stem cell 83 Test using differential gene expression of differentiation-related genes

Chapter 6 Evaluation of developmental toxicant identification using gene expression profiling in embryonic stem cell differentiation cultures

Chapter 7 Concentration-dependent gene expression responses to 123 flusilazole in embryonic stem cell differentiation cultures

Chapter 8 Discriminating classes of developmental toxicants using gene expression profiling in the Embryonic Stem cell Test

Chapter 9 Gene set assembly for quantitative prediction of developmental toxicity in the Embryonic Stem cell Test

Chapter 10 Identification by gene co-regulation mapping of novel genes involved in embryonic stem cell differentiation

Chapter 11 Summary and general discussion

Nederlandse samenvatting

References

Dankwoord

List of publications

Curriculum Vitae 261

Color figures 
Abbreviations 



\begin{tabular}{|c|c|c|c|}
\hline 5-FU & 5-fluorouracil & MBP & Monobutyl phthalate \\
\hline 6-AN & 6-aminonicotinamide & $\mathrm{MeHg}$ & Methylmercury chloride \\
\hline AUC & Area Under the Curve & MEHP & Monoethylhexyl phthalate \\
\hline BrdU & 5-bromo-2'-deoxyuridine & MMP & Monomethyl phthalate \\
\hline CBZ & Carbamazepine & MTX & Methotrexate \\
\hline CDF & Chip Description File & $\mathrm{NCBI}$ & National Center for \\
\hline CON & Control group & & Biotechnology Information \\
\hline \multirow[t]{2}{*}{ CTD } & Comparative Toxicogenomics & NIF & Nitrofen \\
\hline & Database & NRC & National Research Council \\
\hline \multirow[t]{2}{*}{ DMEM } & Dulbecco's Modified Eagle & OECD & Organisation for Economic \\
\hline & Medium & & Co-operation and Development \\
\hline DMSO & Dimethyl sulfoxide & PBS & Phosphate-buffered saline \\
\hline EB & Embryoid body & PC & Principal component \\
\hline \multirow[t]{3}{*}{ ECVAM } & European Centre for the & PCA & Principal component analysis \\
\hline & Validation of Alternative & PenG & Penicillin G \\
\hline & Methods & RA & Retinoic acid \\
\hline ESC & Embryonic stem cells & $\mathrm{REACH}$ & Registration Authorization and \\
\hline EST & Embryonic Stem cell Test & & Evaluation of Chemicals \\
\hline FDR & False Discovery Rate & RIN & RNA Integrity Number \\
\hline FLU & Flusilazole & RMA & Robust Multichip Average \\
\hline FR & Fold ratio & $\mathrm{ROC}$ & Receiver Operating \\
\hline GO & Gene Ontology & & Characteristic \\
\hline GOID & Gene Ontology identifiers & RT-PCR & Real-time polymerase chain \\
\hline GSEA & Gene Set Enrichment Analysis & & reaction \\
\hline HEX & Hexaconazole & SACC & Saccharin \\
\hline LIF & Leukemia inhibitory factor & TDI & Triadimefon \\
\hline LOOCV & Leave-one-out cross-validation & VPA & Valproic acid \\
\hline MAA & Methoxyacetic acid & WARF & Warfarin \\
\hline
\end{tabular}


Dorien A.M. van Dartel

In part published in Comprehensive Toxicology: Developmental Toxicology (2010) 293-307 
CHAPTER 1

General introduction 



\section{Alternative methods in developmental toxicology}

Current developmental toxicity testing is via in vivo experimentation

The importance of testing for developmental toxicity became evident in the early 1960s due to the discovery of the teratogen thalidomide. Prescribed as a mild sedative to pregnant women, thalidomide exposure resulted in severe congenital malformations in more than 10,000 children [1]. This disaster illustrated the potential impact of prenatal chemical exposures and emphasized the importance of hazard identification of these compounds. In the 1980s, toxicity test guidelines established at the Organisation for Economic Co-operation and Development (OECD) have been implemented in order to improve the detection of the developmental toxic properties of compounds. These OECD guidelines cover the critical stages of reproduction and development, i.e. maturation, mating and gestation, and are based on animal experimentation. Specifically regarding the assessment of developmental toxicity, at least two generations of animals are needed. Consequently, these studies are costly, time-consuming, and require considerable numbers of laboratory animals. Because the potential impact of developmentally toxic effects of a compound is significant, studies evaluating developmental toxicity are of great importance. However, the continued use of relatively large numbers of experimental animals for toxicity testing raises ethical issues and is inconsistent with growing emphasis on reduction, replacement and refinement of animal use [2]. Moreover, the number of experimental animals that are needed for the assessment of potential toxicity is expected to increase because of the new EU regulation for Registration Authorization and Evaluation of Chemicals (REACH) [3]. Altogether, this motivates the design of alternative methods that could predict developmental toxicity at an early stage of compound development while requiring only a small volume of compound and a minimal number of experimental animals.

The Embryonic Stem cell Test as alternative testing model

Blastocyst-derived murine pluripotent embryonic stem cells (ESC) can be induced to differentiate in culture into a wide variety of cell types, including cardiomyocytes. This differentiation pathway is the basis of the Embryonic Stem cell Test (EST), which was first described by Spielmann et al. [4]. Cardiomyocytes have been selected as target cells due to the easy visual identification of contracting cells in differentiated ESC cultures. In addition, the in vitro differentiation into cardiomyocytes has been extensively investigated and standardized. Using the hanging drop culture technique [5], ESC form multicellular aggregates, named embryoid bodies (EB). EB resemble the egg-cylinder stage of a 5-day-old embryo. Within the EB, the three germ layers endo-, ecto- and mesoderm can develop [6]. Further differentiation into cardiomyocytes can be induced by cultivation in suspension culture for two days followed by plating onto tissue culture plates. This 
specific culture method results in EB with foci of contracting cardiomyocytes (Figure 1). Inhibition of differentiation is determined by microscopical inspection of contracting cardiomyocytes at day ten of differentiation. The effect of compound exposure on differentiation of ESC into cardiomyocytes is regarded as a measure of the embryotoxicity of compounds. A standard protocol for the EST is available from the European centre for the validation of alternative methods (ECVAM) website [7].

Stem cell research dates back to the early 1970s, when pluripotent embryocarcinoma cells (ECC), the stem cells derived from teratocarcinomas, were established as cell lines $[8,9]$. ECC can be induced to differentiate in culture into a wide variety of tissues. At first, these cells served as a model system to study early differentiation in the mammalian

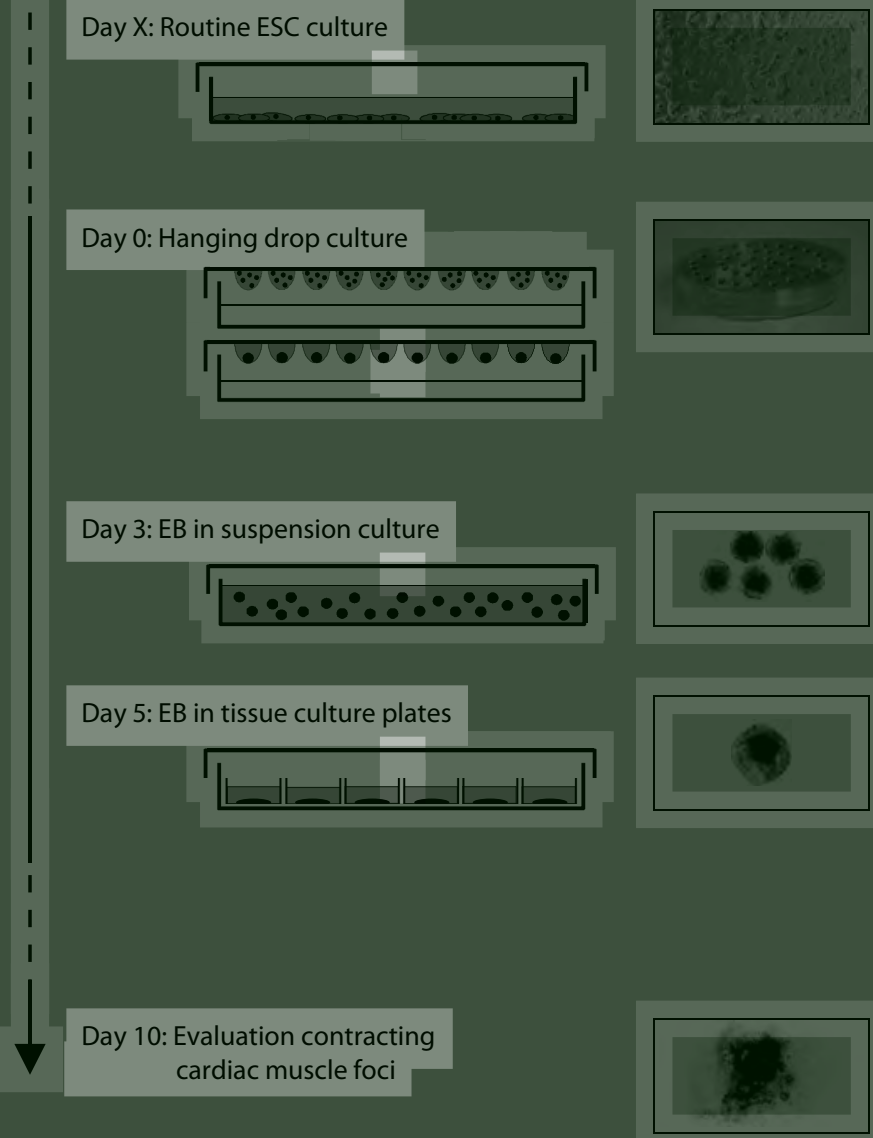

Figure 1. Differentiation protocol for embryonic stem cell (ESC) differentiation. Undifferentiated ESC are cultured as hanging drops. Single cell suspensions aggregate and form embryoid bodies (EB). EB are further cultured in suspension culture. At day 5, EB are plated on tissue culture plates. The morphology of routine culture and untreated $E B$ at day 3,5 and 10 is shown. The classical end point is microscopic evaluation of contracting foci. 
embryo. Later, the interference of teratogenic compounds with ECC differentiation was also studied [10]. ECC, however, may have chromosomal aberrations, may lose their ability to differentiate, or may differentiate in vitro only under specialized conditions and with chemical inducers [11]. After the isolation of the ESC from mouse embryos $[12,13]$ these problems could gradually be overcome and ESC have become the standard for studying cell differentiation in culture. Mouse ESC were initially grown under comparable conditions as ECC, in medium containing bovine serum on a layer of mouse embryonic fibroblasts (called 'feeder cells') to keep ESC pluripotent. The addition of the cytokine leukemia inhibitory factor (LIF) to serum-containing medium allows mouse ESC to proliferate without differentiation, the so-called self-renewal, in the absence of feeder cells [14]. Differentiation of ESC into all cell types of the three primary germ layers can be induced by withdrawal of LIF combined with specific culture conditions sometimes combined with addition of specific growth factors [15].

The EST is unique in that in a relatively simple cell-line-based assay it incorporates the entire differentiation pathways from pluripotent embryonic stem cell to differentiated cells including cardiac muscle cells. The variation of cell types in the assay at the end of culture, including ectoderm-, endoderm- and mesoderm-derived cells, stipulates that cell-cell interactions may play a crucial, though as yet undisclosed, role in the formation of cardiac cells in the EST [6]. Effects of compounds at any stage of the differentiation process can be studied. Furthermore, the test is devoid of experimental animal use and is based on mammalian cells. Recent research has shown that the test may be reproduced with human embryonic stem cell lines $[16,17]$. On the other hand, the current single-lineage readout of the EST may confer a limitation to the assay. The assay by nature provides a reductionistic approach in view of the interaction among regulatory mechanisms and the complexity of pattern formation in embryogenesis which may reduce the applicability domain of the EST.

The EST in Chemical Evaluation

An interlaboratory study was carried out to assess the predictive capacity of the EST. A selection of 20 test compounds, assigned to three classes as non, weakly and strongly developmental toxicants, on the basis of an in vivo database [18] were tested under blind conditions in four different laboratories from governmental institutions and industry $[19,20]$. An algorithm-based mathematical prediction model was developed to convert the in vitro results into a prediction of in vivo developmental toxicity. The EST achieved an overall accuracy of $78 \%$. All strongly developmental toxicants were correctly identified, but the separation of non from weakly developmental toxicants was not convincing. The selected strongly developmental toxicants represented a limited number of mechanisms of toxicity, most of them affecting cell proliferation. 
Additional testing of developmental toxicants with other toxicological mechanisms could improve the reliability of EST for a broader range of compounds. The predictivity of the was also studied for in-house and marketed pharmaceuticals [21, 22]. Although receptor-mediated compounds were excluded from the validation set, the overall accuracy for marketed pharmaceuticals showed to be $85 \%$ but for in-house compounds only $53 \%$, mainly due to bad separation of non from weakly developmentally toxic pharmaceuticals. Although the EST is a formally validated alternative test method, additional investigations are needed to further define the relevance of the EST [23]. An improved definition of the applicability domain, which is defined as the chemical classes and/or ranges of test method end points for which the model makes reliable predictions [23], is of importance to increase the mechanistic understanding of the EST. This is essential for defining the optimal use of the EST in a test strategy. In addition, the predictive capacity associated with the EST needs to be assessed to ensure reliable toxicity identification.

Trends in improving the EST

To improve the subjective end point evaluation of the EST, it has been suggested to replace the subjective morphological end point evaluation at day ten of differentiation by a reporter gene assay [24]. In this assay green fluorescent protein (GFP) is expressed under control of a-actin, a cardiac-specific promoter. By measuring the change in GFP expression, the effects of compounds on cardiac differentiation can be measured quickly and quantitatively. This method is therefore more suitable for high-throughput screening. Cardiomyocyte differentiation can be objectively quantified with flow cytometry using the cardiac-specific marker proteins Myosin and Actinin [25]. This illustrates the possibility to introduce molecular end points into the EST.

As an alternative to the late cardiomyocyte protein-marker evaluation, gene expression analysis could be used to detect early responses shortly after exposure, which may speed up the testing procedure. By implementation of transcriptome assays into the differentiation assay of the EST, developmentally-important pathways can be revealed that are activated during different phases of differentiation [26-29]. Changes on the gene expression level upon exposure to embryotoxic compounds could show inhibition of differentiation via effects on these specific pathways. In the EST, so far only changes in gene expression induced by compound exposure have been studied in a small selected group of genes [30,31]. Whole genome microarrays have been used in other ESC-based models to identify valproic-acid-induced gene-expression changes [32]. These studies suggest that inclusion of selected target gene-expression markers allows prediction of developmental toxicity. Whole genome microarray analysis could lead to the identification of predictive gene subsets, which can be utilized for routine screening applications. 


\section{The use of toxicogenomics for predictive toxicology}

Technical developments in molecular biology have resulted in tools that are capable of measuring thousands of end points in one single assay, such as transcriptomics that evaluates genome-wide gene expression. Transcriptomic approaches are currently widely employed in research in physiology, pathology and toxicology, and because this technique provides a specific evaluation of gene expression it extends the scale and depth of understanding biological processes. Transcriptomic profiling can be used for the identification of biomarkers that may improve the prediction of specific toxic effects. In addition, identified biomarkers may be used for improved discrimination of compound classes, because exposures that affect similar biological processes will likely share comparable gene expression changes. The application of genomics within toxicology is commonly referred to as toxicogenomics.

Most studies that used toxicogenomics to study prediction of toxicity have been performed in the field of carcinogenesis [33-37]. Many investigations aimed at discriminating carcinogens from non-carcinogens based on their gene expression profile and at identifying biomarker genes for the prediction of carcinogenicity, mostly using in vivo models. Among the first studies in this field, Kramer et al. showed that gene expression profiles of rat livers exposed for five days to carcinogens could be discriminated from those of non-carcinogens [33]. This finding, supported by many others, showed the principle that gene expression profiling may be useful for the identification of carcinogenicity. Other studies proved via cross-validation that small gene sets could successfully be used in the discrimination of carcinogens from non-carcinogens [34, 35]. Later, the great robustness of the use of gene signatures for the prediction of carcinogens was shown by the independent validation of identified gene sets $[36,37]$. Besides the use of toxicogenomics for carcinogenicity prediction, this approach has also shown to be useful in predicting other toxic responses, including endocrine disruption [38], immune toxicity $[39,40]$, and developmental toxicity $[32,41]$.

To date, studies that use in vitro predictive toxicogenomics have been less frequently reported as compared to in vivo studies. It has been shown that gene expression patterns of in vivo and in vitro models upon toxicant exposure were comparable, although differential gene expression levels were less extensive in in vitro cultured cells [42-44]. Also in in vitro toxicity prediction, the focus has been on prediction of carcinogenicity and hepatotoxicity. The ability to distinguish compounds with different mechanisms of action was demonstrated by Burczynski et al. using transcriptomic profiling on exposed HepG2 human hepatoma cells [45]. Later, successful attempts to predict potential toxic effects using transcriptomics have been described for hepatotoxicants [46], (hepato) carcinogenic compounds [47] and sensitizing agents [48]. 
Within the field of developmental toxicology, toxicogenomics has contributed to the understanding of developmental processes and mechanisms of action of a variety of developmental toxicants, such as compounds with estrogenic activity [49, 50], triazoles [51], valproic acid [52] and metals [53, 54]. However, limited studies have been published that addressed the prediction of potential developmental toxicity of existing and novel compounds using in vitro models. In a study of Robinson et al., gene expression profiling in compound-exposed whole embryo cultures showed distinct and relevant alterations in gene expression that precede developmentally toxic responses [55], indicating that transcriptomics-based alternative methods may also be successful to predict developmental toxicity.

Analysis tools for transcriptomic data

Since transcriptomic studies generate large data sets, a challenging task is the interpretation of the gene expression responses that are induced by compound exposure [56]. Normally, first differentially expressed genes are identified, since these genes reflect the biological processes changed by the experimental condition. These genes of interest can be selected by defining threshold values, such as $p$-value or false discovery rate (FDR) sometimes in combination with fold change. The genes differentially expressed above the threshold(s) are then selected for further biological interpretation. Different methods such as cluster analysis [57], principal component analysis (PCA) [58] and selforganizing maps [59] can be useful tools to identify similar gene expression patterns of experimental groups or genes [60]. Because genes reacting similarly to a specific stimulus are expected to be functionally related, further study on biological functions of these genes will be a logical next step in data interpretation.

Information on the biology of individual genes can be derived from manual data-queries in scientific literature and databases, such as Gene Ontology (GO) [61, 62], National Center for Biotechnology Information (NCBI) [63] and the Comparative Toxicogenomics Database (CTD) $[64,65]$. These approaches are often used, but have the drawback that they are time-consuming and depend to a large extent on the researcher's preferences and knowledge of biological mechanisms.

The biological functionality of the regulated genes can also come from analysis programs that calculate overrepresentation of genes annotated to biological processes or molecular function, such as DAVID $[66,67]$ or ErmineJ $[68,69]$. Although these analyses result in lists of genes that share biology, function or location within the cell, they lack the level of biological information that provides the interrelation between genes, the 'ordered assemblies of functions' that collaborate to produce a biological function [70]. Other approaches are available to describe interconnection between genes and proteins in pathway format and to implement these into subsequent analyses. Examples of pathway analysis tools are the publicly available GenMAPP [71] and KEGG [72], and the 
commercial MetaCore [73] and Ingenuity Pathway Analysis Tool [74]. Pathway analysis tools have shown to be useful in numerous studies [75-77], however ESC-related processes such as self-renewal and differentiation are not yet sufficiently covered within these pathway tools. To overcome this limitation, 20 research groups of academia and industry combined their expertise in the 'Functional genomics in embryonic stem cells' (FunGenES) Consortium, resulting in the FunGenES database [78, 79]. This interactive database is based on data of 11 diverse settings representing 67 experimental conditions, and organized with a number of novel features and search tools to promote studies in the biological properties of ESC. So far, several studies have taken advantage of the FunGenES database as a comprehensive resource for studies into ESC biology [80, 81].Another strategy to study functional enrichment of genes is studying group-wise effects on gene sets, for example using Gene Set Enrichment Analysis (GSEA) [82] or T-profiler [83]. These methods include the complete gene expression data to test the expression changes of genes in the context of a functional set. This approach can target gene sets whose constituents show subtle but coordinated expression changes that might not be detected by the individual gene analysis. Although this approach lacks the level of biological information, which provides the interrelation between the genes, this method allows the identification of regulated gene sets of which the individual genes may not meet significance thresholds.

\section{Objective and outline of this thesis}

Objective of the thesis

There is a high demand for in vitro alternatives in the field of reproductive and developmental toxicity. Although the EST has been formally validated, several aspects of the assay need improvement to allow successful implementation of the EST as alternative testing method in regulatory toxicity testing. These aspects include the long culture duration and subjective end point scoring as well as the undefined applicability domain and undefined predictability of the EST model. Genomic technologies have incredibly enhanced the number of end points that can be evaluated simultaneously. The value of genomics within toxicology has been shown by identification of gene expression profiles reflective of compound-induced toxicity, such as those characteristic for carcinogenic compounds. It is expected that the implementation of genomics into the EST will provide a more detailed end point evaluation as compared to the classical morphological scoring of differentiation cultures. Therefore, genomics may contribute to the definition of the applicability domain of the EST leading to an improved predictive ability of the assay. Furthermore, the use of toxicogenomics may shorten the culture duration and enable objective end point evaluation. Therefore, the aim of this research is to optimize 
the EST for identifying potential developmental toxicity of compounds using transcriptomics. An overview of the experiments described in this thesis is provided in Figure 2. This transcriptomics-based EST may ultimately contribute to reduction and replacement of animal experimentation within developmental toxicity testing.

Outline of the thesis

A more detailed description of processes active during ESC differentiation may contribute to an improved definition of the relevance of the EST. In this regard, the intertwined relation of proliferation and differentiation in ESC differentiation has been extensively studied in chapter 2 . The results were used to optimize the experimental procedures of the EST leading to a more specific evaluation of compound-induced effects on ESC differentiation, which is considered more specific for developmental toxicants.

To characterize ESC differentiation on the gene expression level, differential gene expression changes were identified by transcriptomics using cultures sampled at 6,12 , and $24 \mathrm{~h}$ after the initiation of ESC differentiation as described in chapter 3 . All genes up-regulated during this phase of ESC differentiation were combined in a gene set. Furthermore, gene expression analysis was performed using ESC differentiation cultures exposed for 6,12 , or $24 \mathrm{~h}$ to the developmental toxicant monobutyl phthalate (MBP) to provide a proof-of-principle that developmental toxicants can be identified via their altered gene expression profile in ESC differentiation cultures.

Chapter 4 describes the gene expression of differentiating ESC 0, 24, 48, 72 and 96h after initiation of differentiation to further characterize ESC differentiation. A novel PCAbased approach, the 'differentiation track' approach, was introduced to describe normal ESC differentiation in the EST. Furthermore, gene expression profiles of ESC differentiation cultures exposed to the developmental toxicants MBP and 6-aminonicotinamide were analyzed by transcriptomics to study the possibility of identification of compoundinduced differentiation-modulating activities by deviation from the 'differentiation track'.

The topic of chapter $\mathbf{5}$ is the optimization of the 'differentiation track' approach for the identification of developmental toxicants. Within this study, the transcription effects of six compounds were tested in the EST. The gene expression data was used to identify a novel biomarker gene set for developmental toxicity prediction using the'differentiation track' method. Leave-One-Out cross validation was applied to validate this optimized approach.

In chapter 6, the identification of potential developmental toxicants using the 'differentiation track' approach in the EST with predefined gene sets, described in chapter 3 and 5, was evaluated. For this evaluation, ESC differentiation modulation of compounds with diverse modes of action and teratogenic effects were tested at single effective concentrations. Furthermore, the data revealed a correlation between ESC differentiation inhibition and identification of developmental toxicants. Since ESC differentiation 
Chapter 2
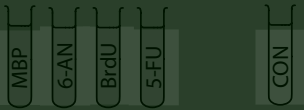

Evaluation proliferation and differentiation

Chapter 3

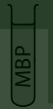

$6,12,24 \mathrm{~h}$

Chapter 4

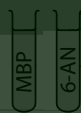

$24,96 h$

Chapter 5

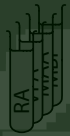

$24 \mathrm{~h}$

Chapter 6

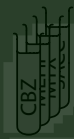

$24 \mathrm{~h}$

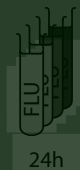

$24 \mathrm{~h}$

Chapter 7

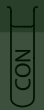

$6,12,24 \mathrm{~h}$

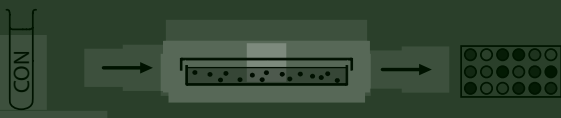

$0,24,48,72,96 \mathrm{~h}$

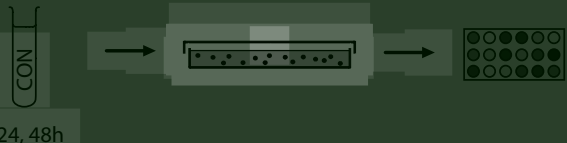

$0,24,48 \mathrm{~h}$

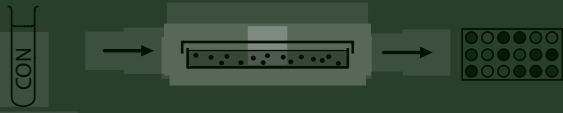

$0,24,48 \mathrm{~h}$

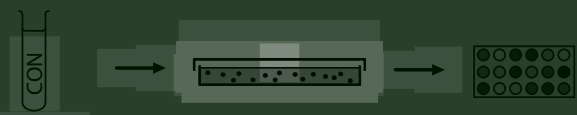

$0,24,48 \mathrm{~h}$

Chapter 8
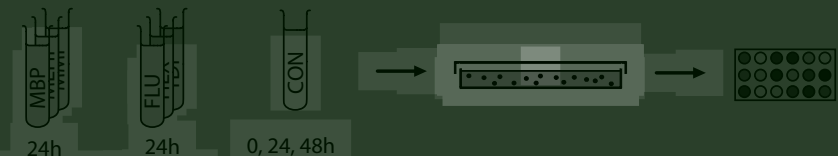

Phthalates Triazoles

$0,24,48 \mathrm{~h}$

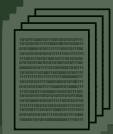

Data chapter 5, 6, 7
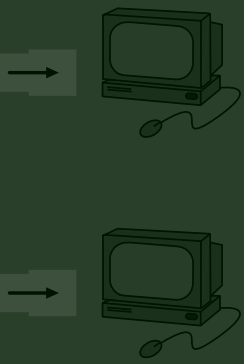

Data literature + database

Figure 2. Overview of experiments described within this thesis. 
inhibition is determined by compound concentration, it was expected that identification of developmental toxicants will be improved by analyzing transcriptomic responses of compounds in a concentration-response design.

Concentration-related gene expression regulation is the subject of chapter 7 . In this chapter, differentiation modulation of the developmental toxicant flusilazole was studied by analyzing concentration-dependent transcriptional responses in differentiating ESC.

The use of gene expression profiling to discriminate compound classes with distinct modes of action was described in chapter 8. Phthalates and triazoles were used in this proof of principle study. Three approaches were used to study class-characteristic gene regulation that may be useful for discrimination of compound classes. In addition, data of the phthalate compounds was used to study the identification of the developmentally toxic phthalates using a category approach.

All data described in chapter 5, 6 and 7 are combined in a new integrated analysis that is described in chapter 9 . This study aimed at identifying a gene set that allows for improved prediction of developmental toxicity based on all available data. Random gene sets were sampled and evaluated to identify the best performing genes for prediction of developmental toxicity using the 'differentiation track' approach.

Chapter $\mathbf{1 0}$ explores a new approach that takes gene sets from literature as input and uses gene co-occurrence for mapping a co-regulation network. Using this approach, novel genes, previously not associated with stem cell or organ development, were identified. The time-series described in chapter 4 were used to visualize the differentiation process.

Chapter 11 contains a summary of the results and the general discussion on the use of the EST combined with toxicogenomics for the identification of developmental toxicants. Furthermore, additional improvements necessary to bring the EST assay to the next level that will allow regulatory acceptation of the test were discussed. 

Dorien A.M. van Dartel, Nicole J.L. Zeijen, Liset J.J. de la Fonteyne, Frederik J. van Schooten, Aldert H. Piersma

Reproductive Toxicology (2009) 28:254-61 
CHAPTER 2

Disentangling cellular proliferation and differentiation in the Embryonic Stem cell Test, and its impact on the experimental protocol 


\begin{abstract}
The mouse Embryonic Stem cell Test (EST) was designed to predict embryotoxicity based on the inhibition of the differentiation of embryonic stem cells (ESC) into beating cardiomyocytes in combination with cytotoxicity data in monolayer ESC cultures and 3 T3 cells. In the present study, we have tested a diverse group of chemicals in EST, applying different exposure durations, in an attempt to discriminate between effects on proliferation and differentiation within the EST protocol. Chemicals tested were monobutyl phthalate (MBP), 6-aminonicotinamide (6-AN), 5-fluorouracil (5-FU) and 5-bromo2'-deoxyuridine (BrdU). We showed that 5-FU and BrdU behaved principally different from MBP and 6-AN. 5-FU and BrdU specifically affected cell proliferation during the first three days of the EST protocol, as shown by EB size, protein concentration and cell cycle stage analysis. In addition, we studied the differentiation state of cells in the EST protocol with time to elucidate the transition of pluripotent ESC to more differentiated cell types. Analysis by flow cytometry of the pluripotency marker SSEA-1 in EST showed that although total SSEA-1 positive cells remained unchanged up to and including day 5 , the signal intensity already decreased from day 3 onwards. Furthermore, RT-PCR data showed an up-regulation of the mesodermal marker $T$ at day 3, whereas the cardiac muscle marker Myh6 was up-regulated from day 5 onwards. These findings confirm that proliferation and differentiation of ESC in the EST are highly intertwined processes. Based on these findings we suggest an amended EST protocol which could more clearly discriminate between proliferation and differentiation effects of chemicals within the same EST differentiation protocol. This proposal includes a cytotoxicity assessment in EB at day 3 of the EST after day 0-3 exposure, and cardiac muscle foci counts after exposure from day 3-10 in the EST.
\end{abstract}




\section{Introduction}

In the field of developmental toxicology, a range of in vitro methods has been developed with the aim to predict in vivo embryotoxicity whilst reducing animal experimentation. Among the spectrum of these tests are zebrafish embryo, rat whole embryo and organ cultures, and cell line based tests [19, 84-86]. Animal-free cell line based assays include the European centre for the validation of alternative methods (ECVAM) validated Embryonic Stem cell Test (EST) which is probably the most extensively studied test in its class [19]. This method uses murine embryonic stem cells (ESC) which can be induced to differentiate into cardiomyocytes via formation of embryoid bodies (EB). EB bear similarities with the inner cell mass of egg-cylinder-stage embryos and develop cells of meso-, ecto-, and endodermal origin upon continued in vitro culture $[6,87]$. This EB-based differentiation culture is therefore a powerful starting point for studying in vitro inhibition of embryonic cell differentiation, that may be used for the prediction of embryotoxicity of chemicals. In the EST, the extent of cardiomyocyte differentiation is microscopically evaluated after 10 days culture. In the standardized assay, cytotoxicity and differentiation inhibition data of the selected compound are combined to predict the degree of embryotoxicity using a mathematical prediction model. The interpretation of EST results is therefore highly dependent on the relative potency of a chemical as regards its cytotoxicity versus its differentiation inhibition. It is known that during differentiation of ESC, proliferation gradually decreases and concomitantly differentiation increases $[88,89]$. Consequently, when specific effects on differentiation are of interest, exposure should perhaps start later in the protocol. This would avoid inhibition of proliferation in the EB formation stage during the first three days of the protocol and may provide a more specific differentiation-mediated response, less influenced by effects on cell proliferation, and possibly improving prediction.

To test this hypothesis, we selected four compounds based on the ECVAM test compound list for validation of embryotoxicity tests [90], and exposed the cells during different exposure periods. These compounds were selected on the basis of their in vivo and in vitro potency and because they do not need metabolic activation. Monobutyl phthalate (MBP) [91-93] and 6-aminonicotinamide (6-AN) [94-96] were selected as specific embryotoxicants, 5-fluorouracil (5-FU) $[97,98]$ and 5-bromo-2'-deoxyuridine (BrdU) [99-101] were selected as known proliferation inhibitors. We demonstrate that in EST 5-FU and BrdU behave principally different from MBP and 6-AN in terms of cytotoxicity versus differentiation inhibitory potencies. In addition, we show new data indicating that differentiation in the EST is a gradual process, detectable as early as day 3 in the protocol, with beating muscle foci detectable at day 7 of culture. We discuss the possible consequences of these findings for the EST exposure protocol. 


\section{Materials and methods}

Pluripotent embryonic stem cell culture

Pluripotent mouse D3 embryonic stem cells (ESC; ATCC, Rockville, MD) were routinely subcultured every 2-3 days and grown as a monolayer in complete medium, consisting of Dulbecco's Modified Eagle Medium (DMEM; Gibco BRL, Gaithersburg, MD) supplemented with $20 \%$ fetal bovine serum (Hyclone, Logan, UT), $1 \%$ non-essential amino acids (Gibco BRL, Gaithersburg, MD), 1\% penicillin/ streptomycin (Gibco BRL, Gaithersburg, MD), 2 mM L-glutamine (Gibco BRL, Gaithersburg, MD) and $0.1 \mathrm{mM} \beta$-mercapto-ethanol (Sigma-Aldrich, Zwijndrecht, The Netherlands). Leukemia inhibitory factor (LIF; Chemicon, Temecula, CA) was added directly to the culture disk in a final concentration of 1000 units $/ \mathrm{ml}$. The cells were maintained in a humidified atmosphere at $37^{\circ} \mathrm{C}$ and $5 \% \mathrm{CO}_{2}$.

Cardiomyocyte differentiation culture

Embryoid body (EB) formation was used as for the initial step for ESC differentiation cultures. EB were obtained via hanging drop culture in complete medium without LIF, carried out according to De Smedt et al. [102]. In brief, stem cell suspensions $\left(3.75 \cdot 10^{4}\right.$ cells $/ \mathrm{ml}$ ) were placed on ice before the set up of the culture (Figure 1). Drops $(20 \mu \mathrm{l})$ containing 750 cells were placed onto the inner side of the lid of a Petri dish filled with phosphate buffered saline (PBS; Gibco BRL, Gaithersburg, MD) and incubated at $37^{\circ} \mathrm{C}$, $90 \%$ relative humidity and $5 \% \mathrm{CO}_{2}$. After 3 days of hanging drop culture $\mathrm{EB}$ had formed and these were subsequently transferred to bacterial Petri dishes (Greiner Bio-one, Frickenhausen, Germany). On day 5, 24 EB were plated one per well into 24-well tissue culture plates (TPP, Trasadingen, Switzerland). Differentiation was determined microscopically at day 10 of differentiation by inspection of EB outgrowths into contracting myocardial cells. EB were considered as cardiomyocyte positive if at least one contracting focus was present. The number of positive EB was expressed as fraction of total EB examined.

Derivation of concentration-response curves and determination of $\mathrm{ID}_{50}$ concentrations

Differentiation cultures were exposed to monobutyl phthalate (MBP; TCl Europe, Zwijndrecht, Belgium; CAS No. 131-70-4), 6-aminonicotinamide (6-AN; Fluka, Buchs, Switzerland; CAS No. 329-89-5), 5-fluorouracil (5-FU; Sigma-Aldrich, Zwijndrecht, The Netherlands; CAS No. 51-21-8), or 5-Bromo-2'-deoxyuridine (BrdU; Sigma-Aldrich, Zwijndrecht, The Netherlands; CAS No. 59-14-3). In addition, appropriate solvent concentrations were tested in control differentiation cultures. The different exposures started at day $0,3,4,5$ or 7 and continued until the microscopical evaluation of the differentiation into contracting myocardial cells on day 10 (Figure 1). The incubations with MBP ranged from 0.045-4.5 mM diluted in dimethyl sulfoxide (DMSO) (Merck, Darmstadt, Germany), incubations with 6-AN ranged from 0.15-15 $\mu \mathrm{M}$ diluted in Dulbecco's Modified Eagle 


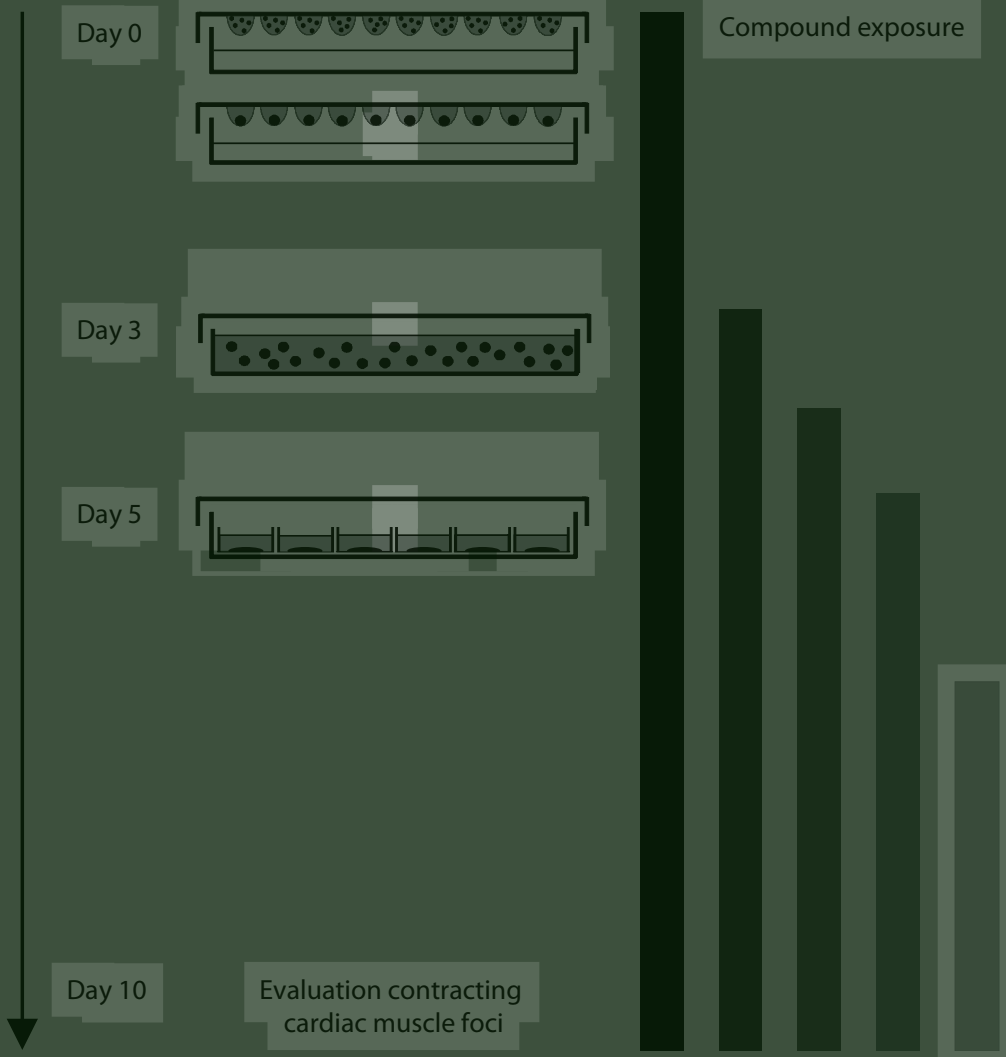

Figure 1. Schematic overview of experimental procedures. Hanging drops, containing single cel suspensions, were set up at day 0 . The single cells form embryoid bodies (EB), which are transferred to suspension culture at day 3. At day 5, EB are plated out in a 24 wells plate. Microscopical evaluation for the presence of contracting foci is performed on day 10.

Medium (DMEM), incubations with BrdU ranged from 0.01-330 $\mu \mathrm{M}$ diluted in DMSO, and incubations with 5-FU ranged from 0.023-2.3 $\mu \mathrm{M}$ diluted in DMEM. Representative concentration-response curves were fitted using the log-logistic model, with PROAST software [103]. Differences of the concentration-response curves between cultures exposed from day 0 compared with exposure from day 3 onwards were assessed using all data regarding these exposure durations. We calculated the critical difference in log-likelihood values of the fit with PROAST software, and curves were considered to be significantly different when this difference exceeded the respective critical value at a $5 \%$-significance level.

The $I D_{50}$ concentration, the concentration at which the number of beating $E B$ was reduced to $50 \%$ of the control, was calculated based on the fit of the curve. Confidence intervals of the $\mathrm{ID}_{50}$ values were obtained by bootstrapping the data 1000 times using PROAST software. 
Resazurin cell proliferation assay

Hanging drops were set up as described above and exposed to a concentration range of MBP, 6-AN, 5-FU or BrdU. After 3 days, 50 EB were harvested per compound concentration, the EB were allowed to precipitate and supernatant was removed. $E B$ were dissociated using non-enzymatic cell dissociation buffer (Gibco BRL, Gaithersburg, MD). Cells were resuspended in $700 \mu \mathrm{l}$ complete medium, and $100 \mu \mathrm{l}$ of this single cell suspension was pipetted per well (in sextuplicate) in a 96 wells plate and resazurin (Promega, Madison, WI) was added to each well. Resazurin dye reduction is a measure for the number of viable cells per well, and was measured after 4 hours of incubation at $37^{\circ} \mathrm{C}$ in the incubator at $530 \mathrm{~nm}$ (excitation) and $590 \mathrm{~nm}$ (emission) using FLUOstar Galaxy microplate reader (BMG Lab Technologies, Offenburg, Germany). After autofluorescence correction of measurements, the resazurin reduction of exposed cells relative to control measurement was calculated and plotted against the test concentrations using PROAST software [103]. For each compound a minimum of five concentrations were tested in three independent runs. The test concentration at which $50 \%$ of the cells were viable as compared to control $\left(\mathrm{IC}_{50}\right)$ was calculated for each compound on basis of the fit of the curve and conficence intervals were obtained by bootstrapping the data 1000 times using PROAST software.

EB diameter measurements

Hanging drops were set up as described above and cells were exposed to the $\mathrm{ID}_{50}$ concentration of MBP, 6-AN, BrdU or 5-FU determined from concentration-response curves of differentiation cultures exposed from day 0 onwards. Appropriate solvent controls were included and penicillin G sodium salt (PenG; Sigma-Aldrich, Zwijndrecht, The Netherlands; CAS No. 69-57-8) was used as negative control. The EB were harvested at the end of the hanging drop culture and photographed using the Olympus IX51 microscope with the UC30 colorview camera (Olympus, Zoeterwoude, The Netherlands). The diameter of $24 \mathrm{~EB}$ per group was measured and difference between the means of exposed EB and control was determined using a one-sided Student's $t$-test and presented as mean $\pm \mathrm{sd}$.

Protein concentration determination

EB exposed to the $I D_{50}$ concentrations of the selected compounds or solvent control were harvested at day 3 for the determination of protein concentration. Per group 100 EB were used, EB were allowed to precipitate and supernatant was removed. EB were dissociated using non-enzymatic cell dissociation buffer (Gibco BRL, Gaithersburg, MD) and washed twice with PBS. Cells were permeabilized using 1\% Triton-X100 (Merck, Darmstadt, Germany), protein concentration was determined using the micro BCA protocol according to the manufacturer's instructions (Perbio Science, Etten-Leur, The 
Netherlands). In short, cells were incubated with BCA working reagent for 30 minutes and absorbance was measured using SpectraMax 190 (Molecular Devices, Sunnyvale, (A) at $562 \mathrm{~nm}$. Differences between the means of exposed cells and control were determined using a one-sided Student's $t$-test and presented as mean $\pm \mathrm{sd}$.

Flow cytometric analysis of DNA content

EB were harvested at day 3 and 400 EB were pooled per group. EB were washed with PBS and dissociated into a single cell suspension by treatment with a non-enzymatic cell dissociation buffer. PBS was added to the single cell suspension and after centrifugation the cells were fixed by addition of $70 \%$ ethanol. After rehydration with PBS the cells were treated for 40 minutes with $0.1 \mathrm{mg} / \mathrm{ml}$ RNase A (Fluka, Buchs, Switzerland) and $50 \mu \mathrm{g} /$ $\mathrm{ml}$ propidium iodide (PI; Sigma-Aldrich, Zwijndrecht, The Netherlands). After centrifugation, cells were resuspended in FACSFlow (Becton Dickinson, Heidelberg, Germany). Fluorescence intensity was determined by flow cytometry on the FACSCalibur (Becton Dickinson, Heidelberg, Germany) in the Fl-2 channel. Non-viable cells and doublets were excluded by appropriate gating and each plot was made up of at least 10,000 viable cells. Data acquisition was performed with CellQuest software (Becton Dickinson, Heidelberg, Germany), and the percentages of G1-, S-, and G2-phase cells were calculated with the ModFit LT 3.0 software program.

Flow cytometric analysis of SSEA-1

The percentage SSEA-1 positive cells was determined in duplicate in pluripotent ESC and at day 3, 4, 5, 7 and 10 of differentiation. Cells were dissociated and $5 \times 10^{5}$ cells were suspended and washed twice with PBS/1 mM EDTA (EDTA: Sigma-Aldrich, Zwijndrecht, The Netherlands). Next, the cells were incubated for 30 minutes with 1:100 mouseanti-SSEA-1 monoclonal antibody (Millipore, Ettenleur, The Netherlands) followed by a 30 minutes incubation with the second antibody, 1:100 goat anti-mouse-FITC (Sigma-Aldrich, Zwijndrecht, The Netherlands). The cells were the then washed twice in PBS/0.5\% BSA/5mM EDTA (BSA, Sigma-Aldrich, Zwijndrecht, The Netherlands). FACSFlow was added for FACS analysis on the FACSCalibur. The FITC-fluorochrome was detected in the Fl-1 channel. Non-viable cells were excluded by appropriate gating and each plot was made up of 10,000 viable cells. ESC, cells lacking primary antibodies, and the fibrosarcoma L929 cell line (ATCC, Rockville, MD) were used as controls. Data acquisition was performed with CellQuest software (Becton Dickinson, Heidelberg, Germany). We distinguished 3 intensities of SSEA-1 positivity as visualized in Figure 6A. Weakly positive staining corresponds with an intenstity of $10^{1}-10^{2}$, medium positive staining with an intensity of $10^{2}-10^{3}$, and strongly positive staining with an intensity of $10^{3}-10^{4}$ detected in the Fl-1 channel. Statistical significance of the results was assessed using the Student's $t$-test. 


\section{Real-time PCR}

ESC were induced to differentiate into beating cardiomyocytes as described above. Biological duplicates of nondifferentiated ESC and cells at days 3, 4, 5, 7 and 10 of differentiation were collected in RNA protect (Qiagen, Venlo, The Netherlands) to stabilize RNA, and total RNA was isolated from the cell cultures using RNeasy protect cell mini kit (Qiagen, Venlo, The Netherlands) with an additional DNase treatment (RNase-Free DNase Set; Qiagen, Venlo, The Netherlands) according to the manufacturer's instructions. The quantity of RNA in each sample was measured using a NanoDrop spectrophotometer (ND1000; NanoDrop technologies, Wilmington, DE) and RNA integrity was determined by automated gel electrophoresis (Bioanalyzer 2100; Agilent technologies, Amstelveen, The Netherlands). cDNA was generated from RNA by using the high-capacity cDNA archive kit containing random hexamer primers (Applied Biosystems, Foster City, CA). mRNA presence was measured with Taqman gene expression assays (Applied Biosystems) on a 7500 Fast Real-Time PCR System, with a two-step PCR procedure according to the manufacturer's protocol. The thermal cycling conditions were: $95^{\circ} \mathrm{C}$ for $20 \mathrm{~s}$ for the first cycle, followed by 40 cycles of $95^{\circ} \mathrm{C}$ for $3 \mathrm{~s}$ and $60^{\circ} \mathrm{C}$ for $30 \mathrm{~s}$. The mRNA markers we used were brachyury ( $T$; Mm01318252_m1), which is one the earliest marker of mesodermal differentiation [104, 105], alpha myosin heavy chain 6 (Myh6; Mm00440359_m1), a marker for cardiac myosine [106], and the housekeeping gene hypoxanthine guanine phosphoribosyl transferase 1 (Hprt1). Hprt1 was selected as housekeeping gene, since its expression was constant under the differentiation process and under compound exposure.

The custom made primers used for Hprt1 were as follows:

$$
\text { 5'-GCC GAG GAT TTG GAA AAA GTG TTT A-3' }
$$

5'-TTC ATG ACA TCT CGA GCA AGT CTT T-3'.

The mRNA expression was normalized to the value of $H p r t 1$ for each reaction according to the comparative Ct method [107] and expressed relative to the expression level in ESC.

\section{Results}

Exposure duration related inhibition of cardiomyocyte differentiation

The EST protocol was carried out with exposure to MBP, 6-AN, 5-FU or BrdU during different time windows. The exposures were started at day $0,3,4,5$ or 7 of the EST protocol and continued until the evaluation of the differentiation assay on day 10 (Figure 1). Concentration-response curves are given in Figure 2. These experiments showed that in most cases a later start of exposure of the differentiating cells to the 4 selected compounds resulted in a decreased inhibition of differentiation towards cardiomyocytes. This included the concentration-response curves of day 0-10 versus day 3-10 exposures 

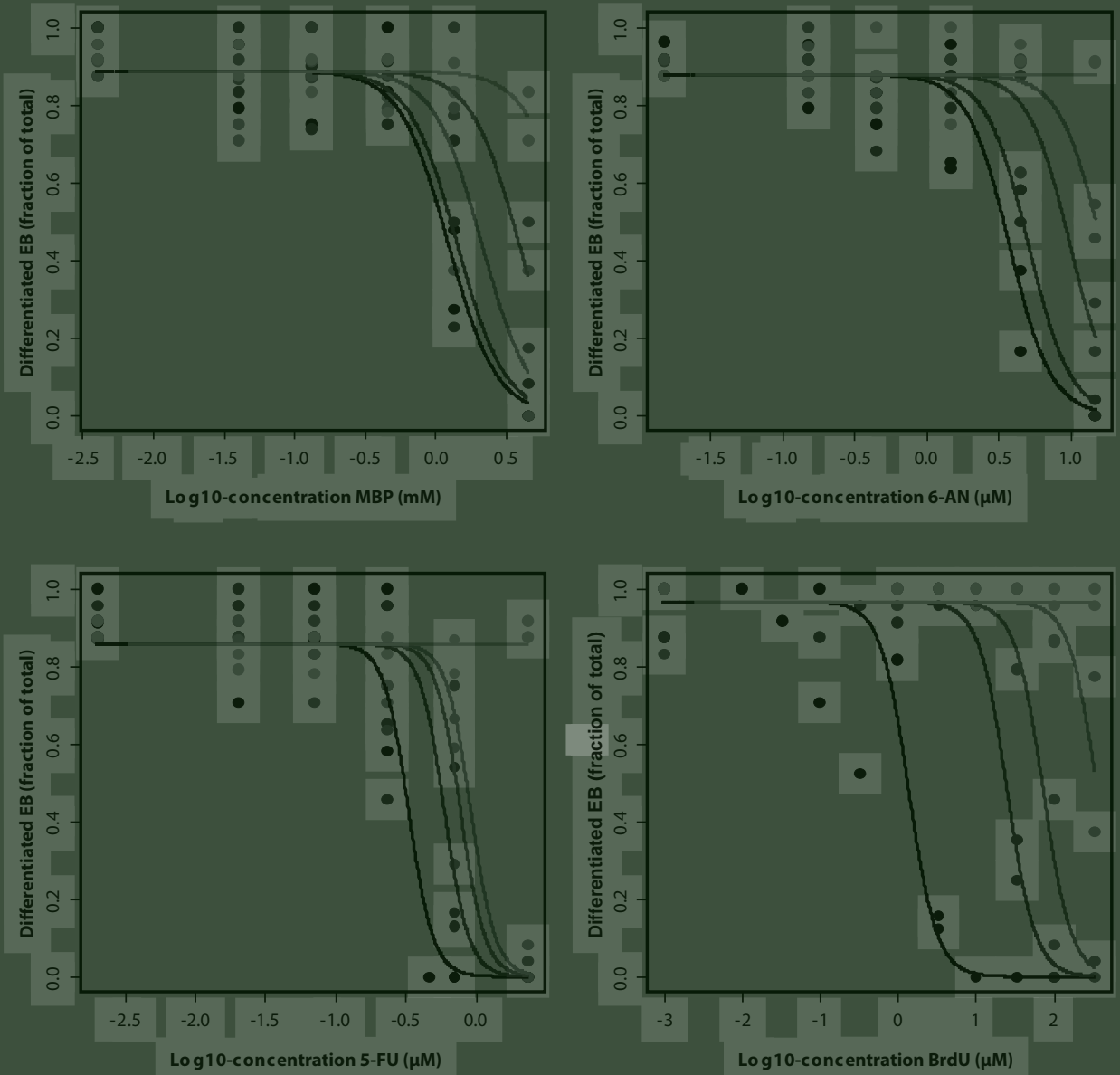

\footnotetext{
Exposure duration

- day 0-10

- day 3-10

- day 4-10

- day 5-10

- day $7-10$
}

Figure 2. Concentration-response curves of inhibition of cardiomyocyte differentiation by monobutyl phthalate (MBP), 6-aminonicotinamide (6-AN), 5-fluorouracil (5-FU) and 5-bromo-2'-deoxyuridine (BrdU) fitted with the log-logistic model on basis of data of at least two independent experiments (solvent controls on the left). Different exposures started at day 0, 3, 4, 5 or 7 and continued up to day 10 (see schematic overview of experimental procedures in Figure 1).

for the cytostatic compounds 5-FU and BrdU, which were significantly different ( $p$ value $<0.05$ ). However, most remarkably, the day 0-10 and day 3-10 exposure curves of the embryotoxicants MBP and 6-AN were not significantly different. This difference in behaviour between the two compound groups suggests a difference in cytotoxic effect in the early EB formation stage of the protocol. 

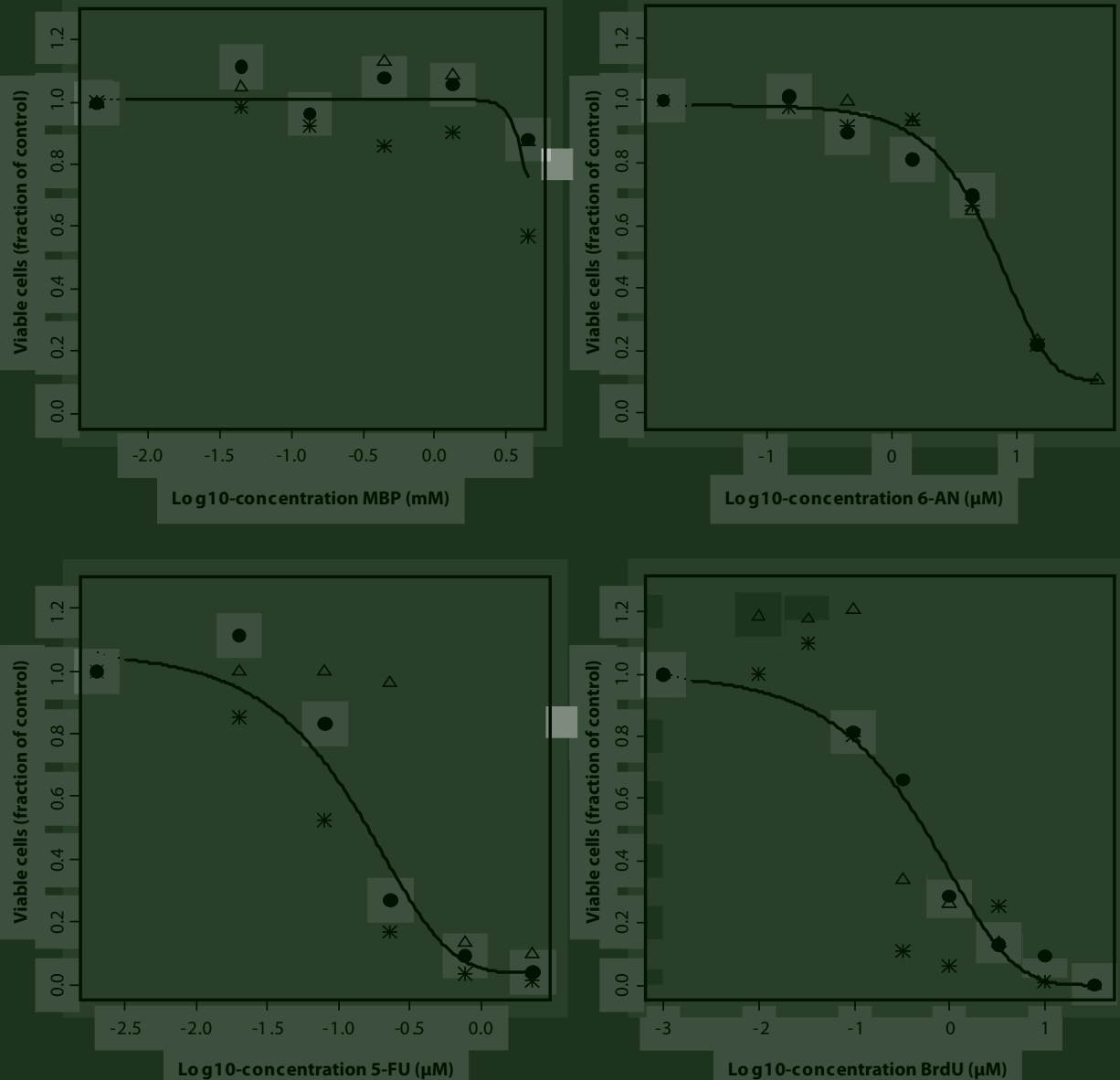

Figure 3. Concentration-response curves of inhibition of cell viability by monobutyl phthalate (MBP), 6-aminonicotinamide (6-AN), 5-fluorouracil (5-FU) and 5-bromo-2'-deoxyuridine (BrdU) (EST protocol, exposure day 0-3) fitted with the log-logistic model on basis of data of three independent experiments with a six technical replicates per experiment. The mean fraction of viable cells is shown per experiment.

Proliferation inhibition during EB formation

We directly investigated inhibition of proliferation of the compounds in the EB formation stage (day 0-3) of the EST protocol (Figure 3). The $\mathrm{IC}_{50}$ concentrations of the proliferation inhibitors 5-FU and BrdU were significantly lower than the $\mathrm{ID}_{50}$ concentration of continuously exposed cells (Table 1), indicating that in the day 0-10 EST protocol, inhibition of proliferation plays a major role in the cardiac differentiation inhibition effects of these compounds. In contrast, the $\mathrm{IC}_{50}$ concentrations of MBP and 6-AN were significantly higher compared with the $I_{50}$ concentrations of continuously exposed cells, suggesting a more specific differentiation-inhibitory effect for these compounds. 
Table 1. Summary of $\mathrm{IC}_{50}$ (EST protocol, exposure day $0-3, \mathrm{n}=3$ ) and $\mathrm{ID}_{50}$ concentrations (EST protocol, exposure day $0-10, n \geq 2$ ) of monobutyl phthalate (MBP), 6-aminonicotinamide (6-AN), 5-fluorouracil (5-FU) and 5-bromo-2'-deoxyuridine (BrdU). Data is represented as mean (95\% Cl).

\begin{tabular}{ccc}
\hline Compounds & $\mathbf{I C}_{\mathbf{5 0}}(\mathbf{9 5} \% \mathbf{C l})$ & $\left.\mathbf{I D}_{\mathbf{5 0}} \mathbf{9 5} \% \mathbf{C l}\right)$ \\
\hline MBP & $>4.5 \mathrm{mM}$ & $1.3 \mathrm{mM}(1.3-1.4)$ \\
$6-\mathrm{AN}$ & $7.1 \mu \mathrm{M}(6.9-7.4)$ & $4.1 \mu \mathrm{M}(3.4-5.0)$ \\
$5-\mathrm{FU}$ & $0.15 \mu \mathrm{M}(0.09-0.21)$ & $0.30 \mu \mathrm{M}(0.25-0.35)$ \\
$\mathrm{BrdU}$ & $0.27 \mu \mathrm{M}(0.15-0.47)$ & $0.97 \mu \mathrm{M}(0.65-1.42)$ \\
\hline
\end{tabular}

To further describe these effects, the diameters of $E B$, exposed during hanging drop culture, were measured at day 3 (Figure $4 \mathrm{~A}$ and $4 \mathrm{~B}$ ). The diameters of PenG, MBP and 6-AN exposed cells were not affected. On the other hand, the BrdU exposed cultures showed a non-significant decrease in EB size, and the diameter of 5-FU exposed EB was significantly decreased compared to control ( $p$-value $<0.05$ ). Attempts to perform single cell counts of dissociated EB at day 3 were not informative due to large data variations. However, whereas protein concentrations of EB on day 3 after exposure to PenG, MBP

A
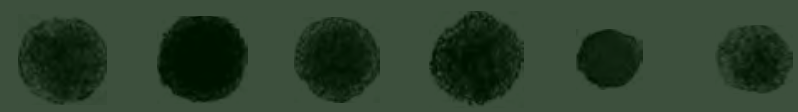

B

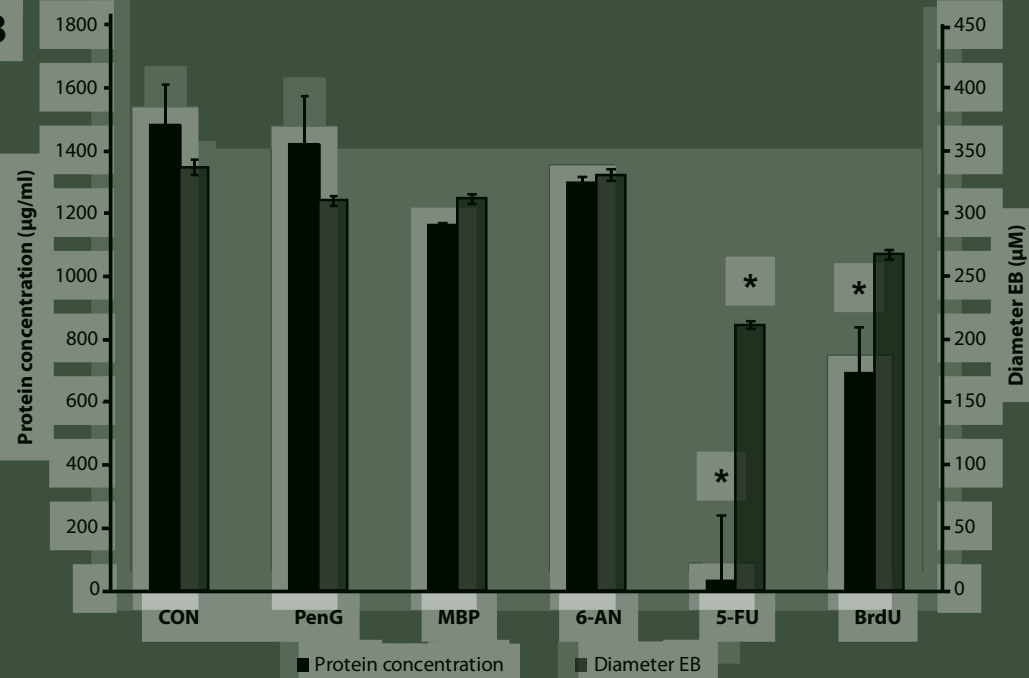

Figure 4. Representive pictures of control and exposed EB at day 3 of the culture (magnification 20x) (A). Protein concentrations (black) and diameter (grey) of non-exposed EB (CON), and EB exposed to Penicillin G (PenG), monobutyl phthalate (MBP), 6-aminonicotinamide (6-AN), 5-fluorouracil (5-FU) or 5-bromo-2'deoxyuridine (BrdU) (B). Bar graphs represent mean $\pm \mathrm{sd}, \mathrm{n}=2$ for EB protein concentration measurements, $\mathrm{n}=\mathbf{2 4}$ for EB diameter measurements. Asterisks indicate statistical significant changes between exposed and control cells ( $p$-value<0.05). 


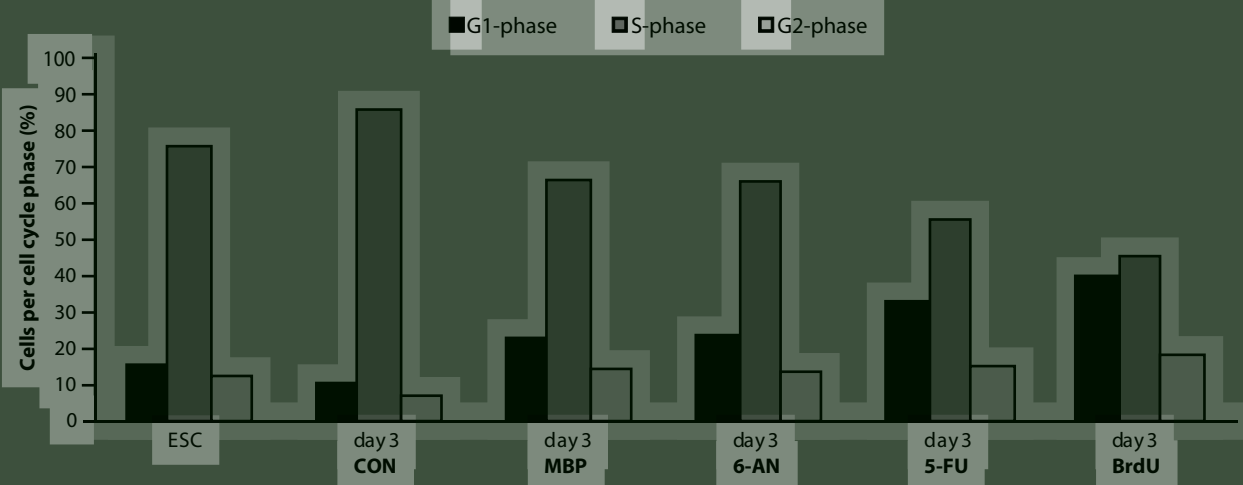

Figure 5. Cell cycle distribution of embryonic stem cells (ESC) and cells in embryoid body (EB) formation (day 3). During EB formation, cells were were exposed to monobutyl phthalate (MBP), 6-aminonicotinamide (6-AN), 5-fluorouracil (5-FU) or 5-bromo-2'-deoxyuridine (BrdU) or non-exposed (CON). For each analysis 400 EB were pooled.

or 6-AN were not significantly different from control, the protein concentration was significantly lower when cells were exposed to BrdU or 5-FU ( $p$-value $<0.05$ ), as expected (Figure 4B). These findings support the proliferation-inhibitory effects of 5-FU and BrdU during EB formation in the EST protocol and confirm the absence of such an effect of the remaining compounds tested.

Flow cytometric analysis of EB cell cycle structure

As the cell cycle structure is another indicator of proliferation rate, flow cytometry profiles of PI-stained cell samples were compared. The cell cycle structure of cells in standard pluripotent ESC culture appeared to be similar with that of day 3 EB (Figure 5). Furthermore, the cell cycle structure of exposed cells were compared with non-exposed cells at day 3. We observed a slight decrease in the percentage of cells in S-phase after exposure to MBP and 6-AN. However, 5-FU and BrdU exposed cells showed a clear Sphase to G1-phase transition. A decrease of cells in S-phase with a factor 1.5 and 1.9 compared to control was observed after exposure with 5-FU and BrdU, respectively, whilst the fraction of cells G1 phase increased with a factor 2.1 and 2.8 compared to control, respectively. These findings further support a proliferation-inhibitory effect of 5-FU and BrdU, whereas MBP and 6-AN had marginal effects at most.

Transition of pluripotent ESC to differentiated cells

The pluripotent state of the cells in the EST protocol was monitored with time by flow cytometry using a fluorescence-labelled antibody to SSEA-1, a pluripotency marker. The total percentage of SSEA-1 positive cells was decreased at days 7 and 10 ( $p$-value $<0.001$, Figure $6 \mathrm{~B}$ ). To further detail the analysis, we divided the SSEA-1 signal into three categories of intensity, corresponding to orders of magnitude (Figure $6 \mathrm{~A}$ ). In doing so, we ob- 
A

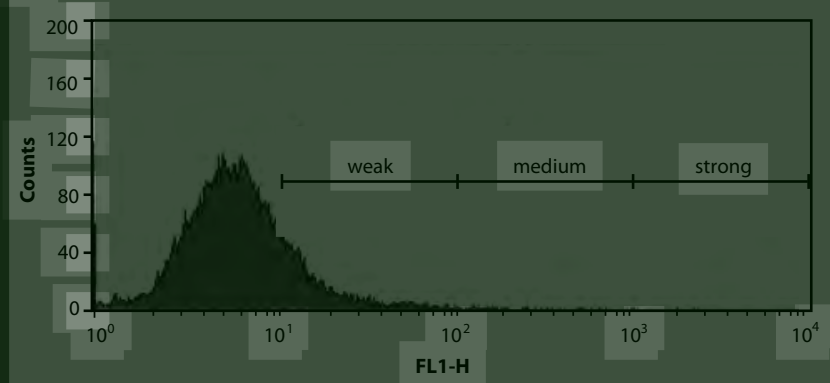

C

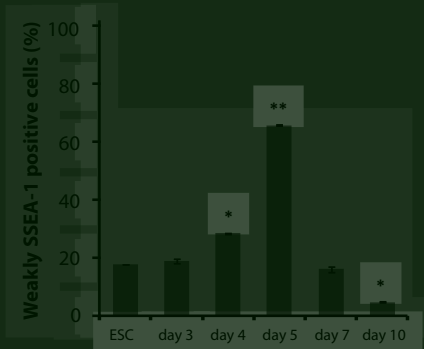

D

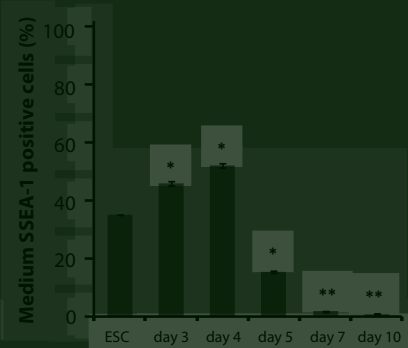

B

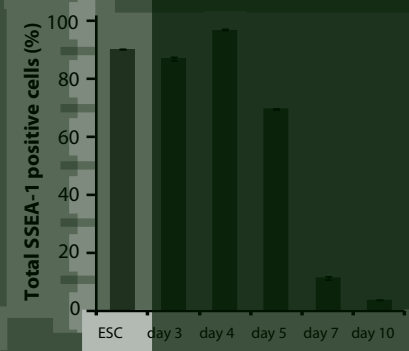

E

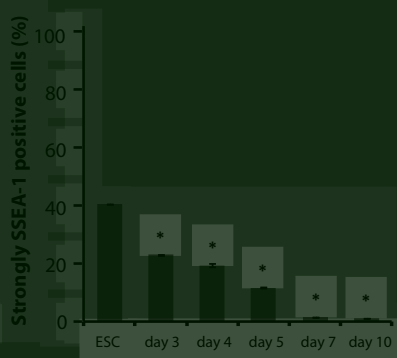

Figure 6. A: Signal-intensity histogram for aspecific binding of FITC-labelled SSEA-1 antibody measured in the FI-1 channel. Signal intensities were divided into three categories: weak: $10^{1}-10^{2}$; medium: $10^{2}-10^{3}$; and strong: $10^{3}-10^{4}$. B-E: Percentage SSEA-1 positive cells per culture day $(n=2)$ (B: all categories; C: weakly positive; D: medium positive; E: strongly positive). Bars represent mean $\pm \mathrm{sd}$. Statistical significance compared with embryonic stem cells $(\mathrm{ESC}){ }^{*}=p$-value $<0.01,{ }^{* *}=p$-value $<0.001$.

served that the SSEA-1 signal per cell already diminished at day 3, as shown by a gradual decrease of the strongly positive cells ( $p$-value $<0.01$, Figure $6 \mathrm{E}$ ). As a consequence the percentages of medium positive cells at day 3 and 4 increased ( $p$-value $<0.01$, Figure 6D), and a high percentage of weak positive SSEA- 1 cells was present at day 5 ( $p$-value $<0.001$, Figure $6 C$ ). At day 7 and 10 almost no SSEA-1 positive cells with a medium or strong signal were detected ( $p$-value $<0.001)$, and only a small population with a weak signal remained.

In view of the above, we wondered whether and how the early decrease in SSEA-1 signal would be accompanied with the occurrence of early and late differentiation markers, respectively. RT-PCR analysis showed that the mRNA of one of the earliest markers for mesodermal differentiation, $T$, was highly expressed at day 3 as compared to its expression in pluripotent ESC. Subsequently, this early marker decreased rapidly with time, with only $23.6 \%$ of the peak value left at day 4 and decreasing further at later time points. In contrast, Myh6, an mRNA marker for cardiac myosin, showed up-regulation from day 5 onwards, with its peak expression at day 7 of cardiac differentiation (Figure 7). At day 10, Myh6 expression was decreased to $76.7 \%$ as compared to day 7 . These 


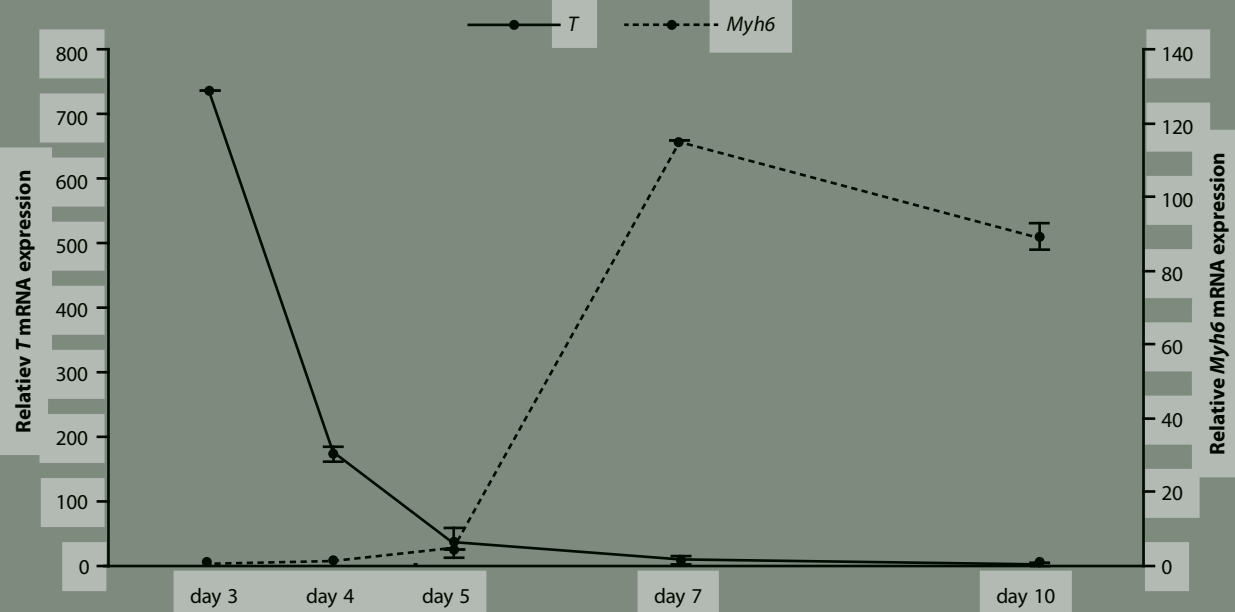

Figure 7. Temporal expression pattern of the mesodermal marker $T$ and the cardiac marker Myh6. Expression level is normalized against Hprt1 and compared with the expression level of ESC. Data is expressed as mean $\pm \mathrm{sd}(\mathrm{n}=2)$.

observations show that as early as day 3 of the EST protocol cells show markers of differentiation, supporting the notion that in the EST proliferation and differentiation are highly intertwined.

\section{Discussion}

The EST was designed to determine the developmental toxicity of chemicals in an animal-free in vitro assay [4]. During an interlaboratory validation programme moderated by ECVAM, the assay was validated by four independent laboratories using 20 chemicals in a blinded study design. This study resulted in a prediction model, defined by independent statisticians. In this model the $\mathrm{ID}_{50^{\prime}}$ the concentration inhibiting beating muscle foci formation by $50 \%$, and two $I C_{50}$ values, the concentrations inhibiting growth by $50 \%$ in pluripotent embryonic stem cell monolayer culture and in the 3 T3 fibroblastoid tumor cell line, were incorporated as parameters. This approach was successful in that it provided a $79 \%$ accuracy among the 20 chemicals tested [19]. Subsequent testing with additional compounds has not delivered the same success rate [21, 22]. In the prediction model of the EST the two most important biological parameters were included, namely cytotoxicity and inhibition of differentiation of ESC. However, in the EST the inhibition of differentiation could be obscured by effects on proliferation of the test compound, since proliferation and differentiation processes both occur in the EST. More specific testing of inhibition of cardiomyocyte differentiation could possibly improve the embryotoxicity prediction. 
In this study, we exposed cells during different exposure windows of the EST protocol and studied inhibition of cardiomyocyte differentiation. We show that the sensitivity of the EST decreases with delay of the onset of exposure, which in itself is not surprising. The remarkable finding of our study is that the difference between day 0-10 and day 3-10 exposure appears to be chemical dependent. Whereas 5-FU and BrdU clearly show additional effects with the 0-10 day exposure protocol, the chemicals MBP and 6-AN do not show significantly different concentration-response curves with both exposure durations. Since 5-FU and BrdU are known cytostatic agents [97-101], and ESC in EB show rapid cell division, inhibition of cell proliferation could explain these differences. Therefore, $I C_{50}$ concentrations of the test compounds were determined in cells exposed during the first 3 days of the EST. For $5-\mathrm{FU}$ and $\mathrm{BrdU} I \mathrm{C}_{50}$ values were significantly lower than their ID $D_{50}$ values, whereas for MBP and 6-AN the IC $C_{50}$ values were significantly higher than their $\mathrm{ID}_{50}$ values. This indicates that BrdU and 5-FU mediated inhibition of cell proliferation observed at day 3 occurs at lower concentrations than inhibition of cardiomyocyte differentiation monitored at day 10. As a consequence, in the enhanced inhibition of cardiomyocyte differentiation when cells were exposed to 5-FU or BrdU from day 0-10 as compared to day 3-10, inhibition of cell proliferation probably plays a major role.

We have further substantiated the day 3 effects by determining EB size, protein content and cell cycle characteristics. Both 5-FU and BrdU caused decreases in EB size and protein content, indicating effects on EB growth. A high proliferation rate is a wellknown characteristic of ESC together with an unusual cell cycle structure composed of a rather large S-phase and a truncated G1-phase [108, 109]. As ESC differentiate, their cell cycle structure changes dramatically and the length of the $\mathrm{G} 1$ phase increases substantially [89, 110]. DNA content analysis showed that the cell cycle profile of day $3 \mathrm{~EB}$ was similar to that of ESC, which suggests the presence of a pure pluripotent cell population at the end of EB formation. Furthermore, the data of DNA content analysis showed that both 5-FU and BrdU exposed cells displayed an S- to G1-phase transition. This effect could be expected as a result of proliferation inhibition. These results confirm our hypothesis that 5-FU and BrdU affect cell proliferation during the first 3 days of cardiomyocyte differentiation. This inhibition of proliferation explains the differences in the concentration-response curves of differentiation cultures exposed to these cytostatic agents from day 0-10 versus day 3-10.

When evaluating the proliferation and differentiation processes in the EST we showed that the percentage of cells positive for SSEA-1 was not decreased until day 7. This supports the hypothesis that the cells start differentiating after completion of EB formation. However, although the total number of SSEA-1 positive cells remain constant up to day 5 , the signal intensity per cell decreased from day 3 onwards (Figure 6E). Analyses of earlier time points were not possible in view of limited cell numbers available in these 
cultures at day 1 and 2. In combination with the RT-PCR results in which the expression of $T$ is observed at day 3 , this implies that the transition from rapidly proliferating pluripotent cells to differentiating cells is a gradual process which seems to start at or even before day 3 of the differentiation culture. The transcription factor $T$ is one of the earliest markers of cardiomyocyte differentiation [104, 105, 111], it is briefly and transiently up-regulated when mesodermal differentiation starts [112]. The first indication of differentiation of cardiac muscle cells appears at day 5, as observed by Myh6 expression. This marker is not found at day 3 and 4 in the EST, confirming that this differentiation event occurs well after EB establishment. In addition, we observed that exposure of cells in the EST from day 7-10 hardly if at all affected the fraction of EB containing contracting cardiomyocyte foci, indicating that cardiomyocyte differentiation is accomplished by day 7. This was confirmed by our RT-PCR results which showed the highest expression level of the cardiac marker Myh6 at day 7. The decrease of the Myh6 expression level at day 10 may be explained by preferential growth of other cell types in the culture, which was also shown by Seiler et al.[25]

It is well known that the formation of cardiac muscle cells is preceded by several differentiation steps, such as endoderm formation and mesoderm induction in an area where ectoderm and endoderm are adjacent $[6,113]$. Our finding that the mesodermal marker $T$ is already expressed at day 3 shows that critical steps in differentiation occur early in the EST. On the other hand, as cardiac myocytes do not begin to appear until day 5 , this allows day 3 as a possible day to start compound exposure in search for specific effects on final cardiac muscle formation that are less influenced by effects on proliferation during EB formation. Our present results with MBP and 6-AN as opposed to 5-FU and BrdU support this notion.

The nature of $\mathrm{IC}_{50}$ determination in the prediction model is a returning discussion

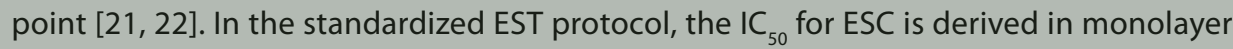
culture, which may have a different sensitivity to chemicals as compared to EB which are used in EST for ESC differentiation. The other cytotoxicity end point in EST, the IC $\mathrm{C}_{50}$ for 3T3 includes a completely different cell line, which may also have a different sensitivity that may or may not be relevant for the prediction of embryotoxicity. To avoid the principal and practical complications of the incorporation of additional culture systems, we have approached the issue of cytotoxicity by studying it within the EST protocol. We show that this approach is able to discriminate among different mechanisms of inhibition of cardiac muscle differentiation.

This study confirms that the proliferation and differentiation of ESC in the EST are co-occurring processes that are not simply separated in time. In vivo these processes are also coupled to coordinate growth and differentiation. The first example of this occurs in the early embryo, which grows at a rapid rate until a critical embryonic mass is generated at the time when gastrulation normally occurs $[114,115]$. Thus, both effects 
on proliferation as well as direct effects on differentiation, can affect embryonic development. When translating this knowledge to the design of the EST, both proliferation and differentiation inhibition are important mechanisms of developmental toxicity and therefore both need consideration as relevant parameters for inclusion in the prediction model. In conclusion, this means that for studying a more specific differentiationmediated response, cells in the EST protocol should be exposed from day 3 onwards. Furthermore, it is worthwile to consider replacing the two $\mathrm{IC}_{50}$ measures currently used in the prediction model for a single $\mathrm{IC}_{50}$ (EB day3) based on the resazurin assay or a similar assay. This would at least provide a comparison between EB inhibition of proliferation and inhibition of cardiac muscle differentiation within the same culture system. These biologically relevant parameters could then be used to predict embryotoxicity and could possibly improve the accuracy of the model. Our recent work relating in vitro $\mathrm{ID}_{50}$ values to in vivo benchmark doses shows promising correlations [116, 117]. On the other hand, any in vitro-in vivo translation will also need to consider differences in compound kinetics in both systems [118]. Future prediction models should probably additionally include such information to further improve prediction of in vivo effects on the basis of in vitro findings.

\section{Acknowledgements}

This study was supported by grant MFA6809 from the Dutch technology society STW. 
Dorien A.M. van Dartel, Jeroen L.A. Pennings, Peter J.M. Hendriksen, Frederik J. van Schooten, Aldert H. Piersma

Reproductive Toxicology (2009) 27:93-102 
CHAPTER 3

Early gene expression changes during embryonic stem cell differentiation into cardiomyocytes and their modulation by monobutyl phthalate 


\section{Abstract}

The Embryonic Stem cell Test (EST) is an in vitro alternative test designed for the prediction of embryotoxicity. The end point of the test is the interference with mesodermderived cardiac muscle differentiation observed under the microscope as beating muscle foci. The relative subjectivity of this end point, as well as the applicability domain and related predictivity need further to be defined to facilitate implementation of the EST into regulatory strategies. The use of transcriptomic techniques to monitor differentiation-related gene expression changes in the EST might improve the EST in each of these aspects. Therefore, we studied the gene expression profile in embryonic stem cells (ESC) in the early phase of differentiation and its modulation by exposure to the well known embryotoxicant monobutyl phthalate (MBP). Cells were exposed from the early embryoid body stage onwards and RNA was collected after 6, 12 and 24h of exposure. Samples were hybridized to spotted microarrays, containing 21,997-mer oligonucleotides. Differential gene expression patterns were analyzed. A total number of 43 genes that were found to be up-regulated in this study as a consequence of induction of cardiomyocyte differentiation were combined in a gene set, named 'Van_Dartel_heartdiff_24h'. Gene set enrichment analysis (GSEA) comparative analysis using multiple gene set collections clearly showed that temporal changes in gene expression were functionally related to cardiomyocyte differentiation. Furthermore, exposure of embryoid bodies to MBP increased expression of pluripotency-, proliferation- and nonmesodermal differentiation-related gene sets, which indicates inhibition of mesodermal differentiation. The inhibition of mesoderm-derived cardiomyocyte differentiation by MBP exposure was most obvious through the down-regulation of our novel gene set identified in this study, 'Van_Dartel_heartdiff_24h', which specifically describes the niche of early cardiomyocyte differentiation. The gene set defined in this study might serve as a starting point for defining a dedicated gene set for early detection of embryotoxicity in the EST. Such a gene set may serve as an improved end point in the EST as compared to morphology, and will allow a more detailed definition of the applicability domain and predictivity of EST. 


\section{Introduction}

Toxicological risk assessment of chemicals is based primarily on animal testing according to internationally agreed protocols. Animal use for hazard identification is especially high in reproductive toxicity testing. Estimations show that under the new European chemicals legislation Registration Authorization and Evaluation of Chemicals (REACH) more than $60 \%$ of experimental animals will be used to assess reproductive toxicity [119]. The need for alternative methods reducing animal use, cost and time is likewise high. In the area of developmental toxicology many alternative tests have been developed during recent decades, none of which has yet acquired regulatory acceptance. The European centre for the validation of alternative methods (ECVAM) validation study of three alternatives [20] has been the most advanced achievement toward regulatory acceptance, and standard protocols are available on the ECVAM web site. However, the relevance and predictivity of these assays are still not sufficiently addressed to allow regulatory implementation.

Of the three validated alternatives for developmental toxicity, the Embryonic Stem cell Test (EST) is the only one which is completely animal-free. Briefly, it employs a continuous murine embryonic stem cell line which is cultured under conditions stimulating differentiation to beating cardiomyocyte foci. The extent of differentiation is scored under the microscope after 10 days culture. Compounds inhibiting differentiation are considered as potential developmental toxicants. Disadvantages of the current protocol include the long duration of culture and the scoring by eye of cardiomyocyte differentiation. Furthermore, the applicability domain in terms of developmental processes incorporated is not well-described in the assay. In addition the limited number of cardiomyocytes at the end of culture is hampering the interpretation of results since other cell types are present and may be affected themselves and/or play a role in inducing cardiomyocyte differentiation. Mesoderm-derived cardiomyocytes are among the first cells acquiring functionality in the embryo.

The mesodermal germ layer develops by induction between the ectoderm and endoderm germ layers. Understanding this complex sequence of steps in the differentiation process requires a comprehensive assessment of the underlying molecular events. Therefore the application of molecular approaches may improve the EST in each of the aspects mentioned above. Seiler et al. have shown that cardiomyocytes can be quantified with flow cytometry on day 7 of culture using specific protein markers [25]. Analysis of gene expression changes using transcriptomics would in principle allow an even earlier detection of differentiation events [120] and in addition may reveal cell type specific effects within the various lineages of embryonic cell differentiation, which may be used to further define the applicability domain and increase the predictivity of the test. 
In this study we exposed the differentiating cells to monobutyl phthalate (MBP), which is the embryotoxic metabolite of butylbenzyl phthalate (BBP) and dibutyl phthalate (DBP). These and other phthalate esters are produced in high volume as plasticizers and are used in vinyl floors, food wraps, cosmetics, medical products, and toys. Consequently, much research has focused on the health effects of these widely distributed chemicals. The most severe adverse effects observed appear to be effects on reproduction and development. Results from a series of animal studies suggest that the monoester metabolite of the parent compound is responsible for adverse reproductive and developmental effects of phthalates [121]. The developmental effects of MBP and its parent compounds depend on the embryonic age at the time of exposure and exposure duration. The effects are mainly on mesodermal structures and include cleft palate, cardiovascular, urogenital, and skeletal malformations [91-93, 122]. Other observed effects are neural tube closure defects such as exencephaly. Since structures affected by MBP are mainly of mesodermal origin, inhibition of (mesodermal) cardiac differentiation by MBP exposure might be demonstrable on the gene expression level. Because the class of phthalates contains both embryotoxic and non-embryotoxic structures, these phthalates are interesting in search for specific gene expression profile changes predictive of embryotoxicity.

Cardiomyocyte differentiation of embryonic stem cells (ESC) was induced by specific culture conditions. The cells were exposed to MBP from the embryoid body (EB) stage onwards. At this stage the EB are in suspension culture and resemble the egg-cylinder stage of a 5-day old embryo [6]. The cells were exposed for 6, 12 or 24 h to study early compound-induced gene expression changes [75, 120]. We demonstrate that early differentiation of ESC can successfully be monitored by transcriptomics. Furthermore, inhibition of cardiomyocyte differentiation by MBP could be demonstrated already after $24 \mathrm{~h}$ of differentiation using a defined gene set specifically related to embryonic development. These findings offer possibilities for further application of the transcriptomic approach to improve the assessment of the applicability domain and the predictivity of the EST, whilst reducing the exposure duration from 10 days to $24 \mathrm{~h}$.

\section{Materials and methods}

Embryonic stem cell culture

Mouse D3 ESC (ATCC, Rockville, MD) were routinely subcultured every 2-3 days and grown as a monolayer in Dulbecco's Modified Eagle Medium (DMEM) medium (Gibco BRL, Gaithersburg, MD) supplemented with 20\% fetal bovine serum (Hyclone, Logan, UT), 1\% non-essential amino acids (Gibco BRL, Gaithersburg, MD), 1\% penicillin/ streptomycin (Gibco BRL, Gaithersburg, MD), 2 mM L-glutamine (Gibco BRL, Gaithersburg, 
MD) and $0.1 \mathrm{mM} \beta$-mercapto-ethanol (Sigma-Aldrich, Zwijndrecht, the Netherlands). Leukemia inhibiting factor (LIF) (Chemicon, Temecula, CA) was added directly to the culture disk in a final concentration of 1000 units $/ \mathrm{ml}$. The cells were maintained in a humidified atmosphere at $37^{\circ} \mathrm{C}$ and $5 \% \mathrm{CO}_{2}$.

Cardiomyocyte differentiation culture

Differentiation of ESC was carried out in hanging drops according to De Smedt et al. [102]. In brief, stem cell suspensions $\left(3.75 \cdot 10^{4}\right.$ cells $\left./ \mathrm{ml}\right)$ were placed on ice before the set up of the culture (Figure 1). Drops $(20 \mu \mathrm{l})$ containing 750 cells were placed onto the inner side of the lid of a Petri dish filled with phosphate buffered saline (PBS) (Gibco $\mathrm{BRL}$, Gaithersburg, MD) and incubated at $37^{\circ} \mathrm{C}, 90 \%$ relative humidity and $5 \% \mathrm{CO} 2$. After 3 days of hanging drop culture embryoid bodies (EB) had formed and these were subsequently transferred to bacterial Petri dishes (Greiner Bio-one, Frickenhausen, Germany). On day 5, EB were plated one per well into 24-well tissue culture plates (TPP,

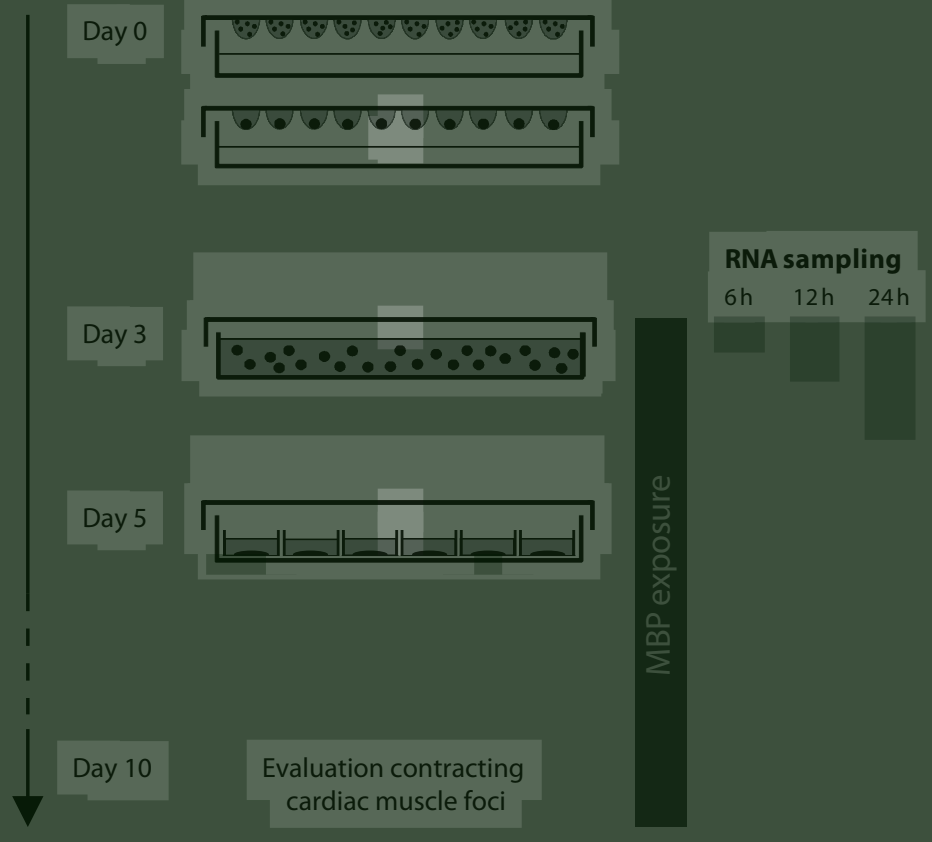

Figure 1. Schematic overview of experimental procedures. Hanging drops, containing single cell suspensions, were set up at day 0 . The single cells form embryoid bodies (EB), which are transferred to suspension culture at day 3. At day 5, one EB is plated per well in a 24-wells plate. At day 10 each EB is microscopically evaluated for the presence of contracting foci. Differentiating cells were exposed from suspension culture onwards. For transcriptomic analysis, samples were isolated after 6, 12 and 24 hours of monobutyl phthalate (MBP) exposure. 
Trasadingen, Switzerland). Differentiation was determined by microscopical inspection of EB outgrowths into contracting myocardial cells at day 10 of differentiation.

Determination of $I D_{50}$ concentration MBP

Differentiation cultures were exposed to monobutyl phthalate (MBP) (CAS No.131-70-4) or $0.25 \%$ dimethyl sulfoxide (DMSO) from suspension culture onwards to day 10 (Figure 1) and differentiation into contracting myocardial cells was microscopically determined. The incubations with MBP ( $\mathrm{TCl}$ Europe, Zwijndrecht, Belgium) ranged from 0.045-4.5 $\mathrm{mM}$ diluted in DMSO. A representative concentration-response curve of MBP was fitted using the log-logistic model, with PROAST software [103]. The $\mathrm{ID}_{50}$ concentration, the concentration which corresponds with the concentration at which $50 \%$ of the EB were inhibited to differentiate towards contracting cardiomyocytes, was calculated based on the fit of the curve.

Resazurin cell viability assay

Hanging drops were set up as described in the section cardiomyocyte differentiation. After 3 days, EB were harvested, dissociated en resuspended in complete medium. Single cells were divided in the wells of a 96 wells plate and resazurin (Promega, Madison, WI) was added to each well. After $4 \mathrm{~h}$ of incubation at $37^{\circ} \mathrm{C}$ in the incubator, resazurin dye reduction was measured at $530 \mathrm{~nm}$ (excitation) and $590 \mathrm{~nm}$ (emission) using FLUOstar Galaxy microplate reader (BMG Lab Technologies, Offenburg, Germany). After autofluorescence correction of measurements, significance of differences in reduction of exposed cells relative to the control measurement was analyzed by Student's T-test, using data of two independent runs.

RNA isolation and processing

For microarray analysis, cells were exposed to the ID50 concentration of MBP (1.35 mM) or $0.25 \%$ DMSO at day 3. EB were harvested 6, 12 and $24 \mathrm{~h}$ later (Figure 1). The cells were directly collected in RNA protect (Qiagen, Venlo, the Netherlands) to stabilize RNA, and total RNA was isolated from the cell cultures using RNeasy protect cell mini kit (Qiagen, Venlo, the Netherlands) with an additional DNase treatment (RNase-Free DNase Set; Qiagen, Venlo, the Netherlands) according to the manufacturer's instructions. The quantity of RNA in each sample was measured using a NanoDrop spectrophotometer (ND1000; NanoDrop technologies, Wilmington, DE) and RNA integrity was determined by automated gel electrophoresis (Bioanalyzer 2100; Agilent technologies, Amstelveen, the Netherlands). 
Transcriptomic profiling

Microarray analysis experiments were performed as described previously by Janssen et al. [123]. In brief, total RNA samples were processed in randomized order using one RNA sample per array and with a mix of all samples as common reference. Of the corresponding RNA samples (7-8 replicates per group) $1 \mu \mathrm{g}$ was amplified using the Amino Allyl MessageAmp II aRNA kit (Ambion, Austin, TX). Next, $5 \mu \mathrm{g}$ of control or exposed sample aRNA was labeled with Cy3, and equal amounts of reference pool with Cy5 (CyDye Post Labeling Reactive Dye Pack; Amersham Biosciences, Buckinghamshire, UK) according to the manufacturer's instructions. Volumes of the labeled and purified aRNA samples, containing $150 \mathrm{pmol}$ of the respective dye, were reduced to $9 \mu$ by vacuum drying. Thereafter, the aRNA was fragmented (RNA Fragmention Reagents; Ambion, Austin, TX), combined with an aliquot of reference pool aRNA and total volume was adjusted to 25 $\mu$ with milliQ water.

Microarray slides were spotted at the Microarray Department of the University of Amsterdam. The slides contain 21,997 65-mer oligonucleotides from the Sigma-Compugen Mouse nucleotide library, as well as appropriate controls and blank spots. The slides were prehybridized at $42^{\circ} \mathrm{C}$ for at least $30 \mathrm{~min}$ in $5 \times \mathrm{SSC}$ (Invitrogen, Breda, the Netherlands), 0.1\% SDS (Ambion, Austin, TX), and 1\% BSA (Sigma-Aldrich, Zwijndrecht, the Netherlands) dissolved in milliQ and sterilized by filtering through a $0.22 \mu \mathrm{m}$ filter (Millipore, Amsterdam, The Netherlands). Subsequently, the slides were washed and dried by centrifugation. Next, hybridization mixture, containing $50 \%$ formamide (Sigma-Aldrich, Zwijndrecht, the Netherlands), 10× SSC, and 0.2\% SDS, was added to the combined sample and reference pool. This mixture was pipetted onto a slide and spread evenly by application of a cover slip. After $16 \mathrm{~h}$ incubation at $42^{\circ} \mathrm{C}$ in the dark in humid hybridization chambers, the slides were washed. Immediately after centrifugation, the microarray slides were scanned using a ScanArray 4000XL microarray scanner (Perkin-Elmer, Waltham, MA) with appropriate laser and photomultiplier tube settings.

Data analysis and statistics

Array Vision software (Imaging Research, St. Catherines, Ontario, Canada) was used to determine median Cy3 and Cy5 signal intensities for each separate spot and background signal. Quality control was performed on raw data by means of a scatter plot and MAplot as well as a normal probability plot to assess signal distribution. The control and blank spots present on the slide were used for quality control, but excluded in the further analysis.

Data normalisation and statistical analysis were carried out using the $\mathrm{R}$ statistical software environment (www.r-project.org). Raw microarray signal data were normalized using a three-step approach as described by Janssen et al.[123]. Genes that were differentially expressed between any of the experimental groups were identified by a 
one-way ANOVA on the normalized data. $P$-values were corrected for multiple testing by calculating the false discovery rate (FDR) according to Benjamini and Hochberg [124]. An FDR significance threshold of 0.20 was used to select differentially expressed genes. Gene expression patterns were visualized by hierarchical clustering (based on Euclidian Distance and Ward linkage) using GeneMaths (Applied Maths, St-Martens-Latem, Belgium). Functional annotation and Gene Ontology (GO) term enrichment were examined with tools on the DAVID website (http://david.abcc.ncifcrf.gov) [67].

Gene set enrichment analysis (GSEA) was performed to discover the differential expression of biologically relevant sets of genes that share common biological function or regulation [82]. Gene sets were considered being significantly affected when the $p$ value was below 0.05 and the FDR below 0.15 . GSEA was followed by molecular concept analysis, in which the gene sets are visualized within a network based on their overlap in genes [125]. The overlap among the gene sets were calculated on the genes that were responsible for a gene set being significant. For this, either the top- $20 \%$ of the ranked gene list for up-regulated genes or the bottom-20\% of the ranked gene list for downregulated genes was used. The significance of overlap between gene sets was calculated based on the binomial distribution using the program Venn-Mapper [126]. Genes that showed significant overlap at $p<0.05$ (Z-value $>1.96$ ) were considered connected in a network that was subsequently visualized using Cytoscape [127].

For the GSEA we used five gene set collections. Two general gene set collections, C2 and GO, were available from open sources. The curated gene sets C2 were from the Molecular Signature Database of the GSEA developers (http://www.broad.mit.edu/ gsea/msigdb/msigdb_index.html), and gene sets for GO (mouse gene symbols) were downloaded from the GO Consortium (http://www.geneontology.org/). In addition, a Tissue Specific gene set collection was created using expression microarray data of tissues [128]. Genes in sets of this collection were considered to be tissue specific when its expression was at least 100-fold higher than the median expression in all tissues. Furthermore, a gene set collection Cell Cycle was included, which contains genes that are specifically up-regulated during a cell cycle stage. These gene sets were taken from the supplementary data of two studies $[129,130]$. The fifth gene set collection, named ESC_Cardiac, contains gene sets derived from microarray studies on gene expression changes during the differentiation of ESC to cardiomyocytes [27, 28, 131-135] or vascular tissues [136]. Gene sets affected by Hmga 1 in ES cells as described by Martinez-Hoyos et al. [137] were also included in the ESC_Cardiac gene set collection. Furthermore, gene sets related to 'ES cells and heart development' or 'ES cells and fetal heart' derived from literature using the text-mining tool Anni 2.0 [138] were included. Finally, the genes that were found to be up-regulated in this study as a consequence of cardiomyocyte differentiation were combined in a gene set, named 'Van_Dartel_heartdiff_24h'. 


\section{Results}

Inhibition of cardiomyocyte differentiation by MBP

A concentration-response curve for MBP in the differentiation assay of the EST is given in Figure 2. The $I D_{50}$ concentration of MBP derived from the curve was rounded to $1.35 \mathrm{mM}$ and this concentration was used in the transcriptomic experiment. At this $\mathrm{ID}_{50}$ viability of exposed cells was $90.7 \pm 3.4 \%$ ( $p$-value<0.05) compared to control as determined using resazurin reduction, suggesting minimal cytotoxicity at the tested concentration.

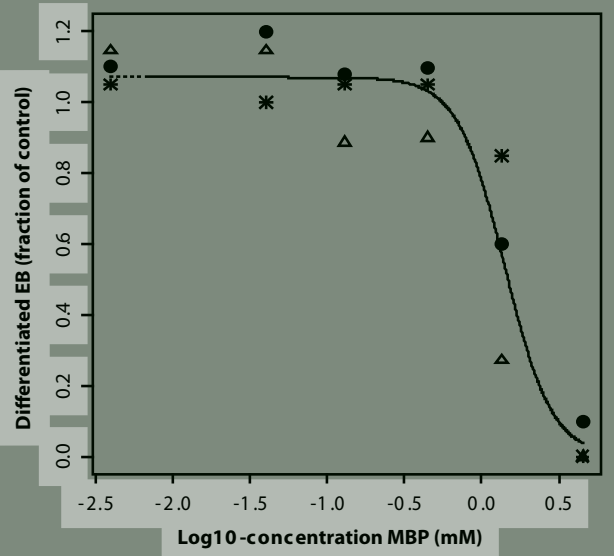

Figure 2. Concentration-response curve of monobutyl phthalate (MBP), fitted with the log-logistic model on the basis of data from three independent experiments. The $I D_{50}$ is calculated on the basis of the fit of the curve.

ESC differentiation related gene expression changes

Gene expression data of all samples were compared by a one-way ANOVA. An FDR of $<0.20$ was applied to select genes that were differentially expressed in at least one of the experimental groups. This yielded 48 genes which were differentially expressed after $24 \mathrm{~h}$ of suspension culture compared to the samples isolated after 6 and $12 \mathrm{~h}$, in the combined control and MBP-exposed samples (Figure 3). In total, 43 genes were upregulated, and 5 genes were down-regulated. Analysis of GO term enrichment showed that these genes are enriched for 'development' ( $p$-value 5.1E-5), 'embryonic development' ( $p$-value 4.0E-3) and 'morphogenesis' ( $p$-value 1.5E-2). All up-regulated genes were included in the new gene set 'Van_Dartel_heartdiff_24h'.

Regulation of gene sets by differentiation of ESC

Data were further analyzed using the GSEA tool testing the following five gene set collections, C2, GO, Tissue Specific, Cell Cycle and ESC_Cardiac and the gene set 'Van_Dartel_heartdiff_24h'. The samples isolated after 6 and $12 \mathrm{~h}$ were combined in this analysis 


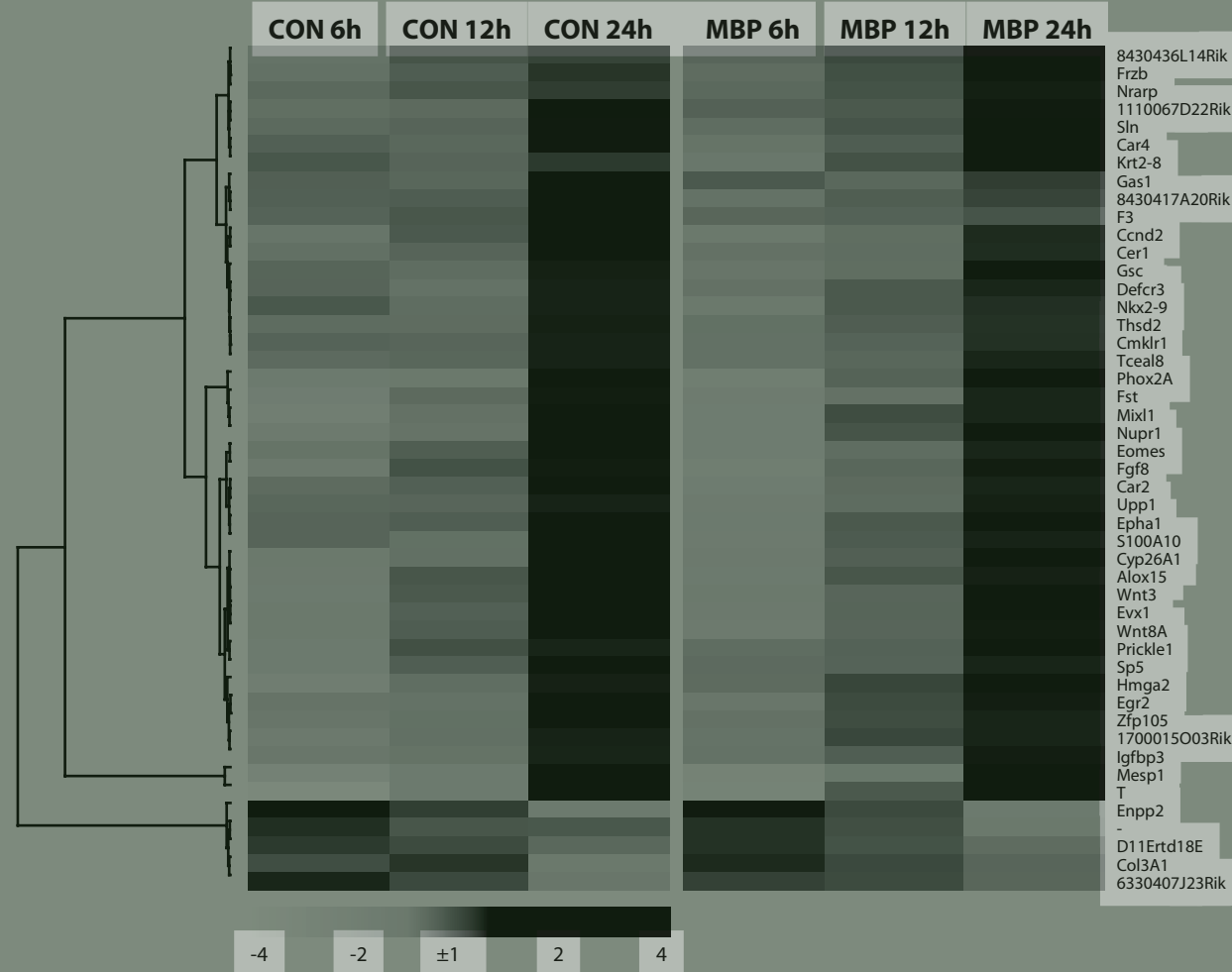

Figure 3. Clustering of differentially expressed genes after $24 \mathrm{~h}$ of suspension culture. Cells were exposed for 6,12 or $24 \mathrm{~h}$ to monobutyl phthalate (MBP) or solvent control (CON). Per group the mean regulation of 7-8 replicates is shown. Gene expression changes are indicated by the color bar, where white represents down-regulation and black up-regulation. For color figure, see Color figures section.

since minimal gene expression changes were observed between these groups (Figure 3). In total, 24 gene sets were found to be regulated by the differentiation process of ESC, as analyzed by comparing the samples after $24 \mathrm{~h}$ of suspension culture to the control samples after 6 and $12 \mathrm{~h}$. These significantly regulated gene sets are presented in Table 1. None of the gene sets from C2, Tissue Specific and Cell Cycle gene set collections were regulated as a consequence of differentiation. Two gene sets of the $\mathrm{GO}$ collection were down-regulated. Furthermore, five GO gene sets were up-regulated, including 'development' and 'somitogenesis'. The majority of the regulated gene sets is from the ESC_Cardiac collection (16/24).

As expected the gene set 'Van_Dartel_heartdiff_24h' is significantly up-regulated, the respective GSEA calculated $p$-value is $<1 \mathrm{E}-3$ and the FDR is $<1 \mathrm{E}-3$ (Table 1 ), and based on a paired t-test, $p$-value $2.9 \mathrm{E}-4$.

The significance of overlap among regulated gene sets in gene content, shown as connections, is visualized in Figure 4. In this molecular concept analysis the high degree of overlap between these up-regulated gene sets from the ESC_Cardiac gene set collection is shown, 
Table 1. Regulated gene sets in ESC cardiomyocyte differentiation cultures.

\begin{tabular}{|c|c|c|c|}
\hline Gene Ontology gene set collection & \# genes/ set & NOM $p$-val & FDR q-val \\
\hline \multicolumn{4}{|l|}{ Downregulated } \\
\hline Glutathione_transferase_activity & 21 & $<1.00 \mathrm{E}-04$ & 7.53E-02 \\
\hline Response_to_unfolded_protein & 37 & $3.96 \mathrm{E}-03$ & $1.21 \mathrm{E}-01$ \\
\hline \multicolumn{4}{|l|}{ Upregulated } \\
\hline Development & 328 & $<1.00 \mathrm{E}-04$ & $1.43 \mathrm{E}-01$ \\
\hline Ribosome & 138 & $<1.00 \mathrm{E}-04$ & $1.16 \mathrm{E}-01$ \\
\hline Structural_constituent_of_ribosome & 160 & $<1.00 \mathrm{E}-04$ & $8.34 \mathrm{E}-02$ \\
\hline Regulation_of_progression_through_cell_cycle & 119 & $1.92 \mathrm{E}-03$ & $1.46 \mathrm{E}-01$ \\
\hline Somitogenesis & 21 & 3.84E-03 & 4.49E-02 \\
\hline \multicolumn{4}{|l|}{ ESC Cardiac gene set collection } \\
\hline \multicolumn{4}{|l|}{ Downregulated } \\
\hline Behfar_pluripotent_markers & 243 & $<1.00 \mathrm{E}-04$ & $<1.00 \mathrm{E}-04$ \\
\hline Behfar_heartdiff_day7 & 264 & $<1.00 \mathrm{E}-04$ & 7.64E-02 \\
\hline \multicolumn{4}{|l|}{ Upregulated } \\
\hline Alexandrovich_early_heartdiff & 28 & $<1.00 \mathrm{E}-04$ & $9.46 \mathrm{E}-03$ \\
\hline Anni_heart_development_and_ES_cell & 117 & $1.95 \mathrm{E}-03$ & 1.37E-01 \\
\hline Anni_heart_development_and_fetal_heart & 64 & $<1.00 \mathrm{E}-04$ & 3.33E-02 \\
\hline Beqqali_heartdiff_day3 & 81 & $<1.00 \mathrm{E}-04$ & $4.34 \mathrm{E}-04$ \\
\hline Beqqali_heartdiff_day3_with_T & 26 & $<1.00 \mathrm{E}-04$ & 3.47E-04 \\
\hline Beqqali_mid_heartdiff & 199 & $<1.00 \mathrm{E}-04$ & $5.26 \mathrm{E}-02$ \\
\hline Beqqali_early_heartdiff & 426 & $1.96 \mathrm{E}-03$ & $9.35 \mathrm{E}-02$ \\
\hline Christoforou_heartdiff_day7-8 & 64 & $<1.00 \mathrm{E}-04$ & $2.84 \mathrm{E}-04$ \\
\hline Faustino_heart/vascular_diff & 65 & $<1.00 \mathrm{E}-04$ & 5.03E-03 \\
\hline Martinez_Hmga1_Ko & 28 & $<1.00 \mathrm{E}-04$ & $9.03 E-04$ \\
\hline TFs_regulating_ESC_lit & 61 & $<1.00 \mathrm{E}-04$ & $3.60 \mathrm{E}-02$ \\
\hline Van_Dartel_heartdiff_24h & 42 & $<1.00 \mathrm{E}-04$ & $<1.00 \mathrm{E}-04$ \\
\hline Wang_Flk1Pos_day8_ & 34 & $<1.00 \mathrm{E}-04$ & 4.43E-04 \\
\hline Wang_Flk1Pos_84h_vs_8d_up & 237 & $<1.00 \mathrm{E}-04$ & $8.52 \mathrm{E}-03$ \\
\hline Wang_Flk1Pos_95h_vs_8d_up & 244 & $<1.00 \mathrm{E}-04$ & $1.68 \mathrm{E}-03$ \\
\hline \multicolumn{4}{|l|}{ Novel gene set (this study) } \\
\hline \multicolumn{4}{|l|}{ Upregulated } \\
\hline Van_Dartel_heartdiff_24h & 42 & $<1.00 \mathrm{E}-04$ & $<1.00 \mathrm{E}-04$ \\
\hline
\end{tabular}

thereby illustrating that these particular gene sets play a role in related biological processes. In addition, the gene set derived from this study showed to have the highest overlap with other up-regulated gene sets. Also, the two down-regulated gene sets from the study of Behfar et al., included in the ESC_Cardiac gene set collection, have a significant overlap with each other. The overlap between the regulated GO gene sets with other sets was minimal. 
REGULATION_OF_PROGRESSION_THROUGH_CELL_CYCLE

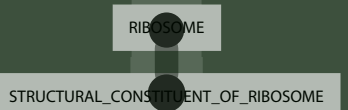

GLUTATHIONE_TRANSFERASE_ACTIVITY

RESPONSE_TO_UNFOLDED_PROTEIN

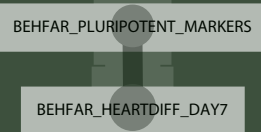

Cardiac gene set; upregulated

Pluripotency gene set; downregulated

Undefined gene set; upregulated

Undefined gene set; upregulated

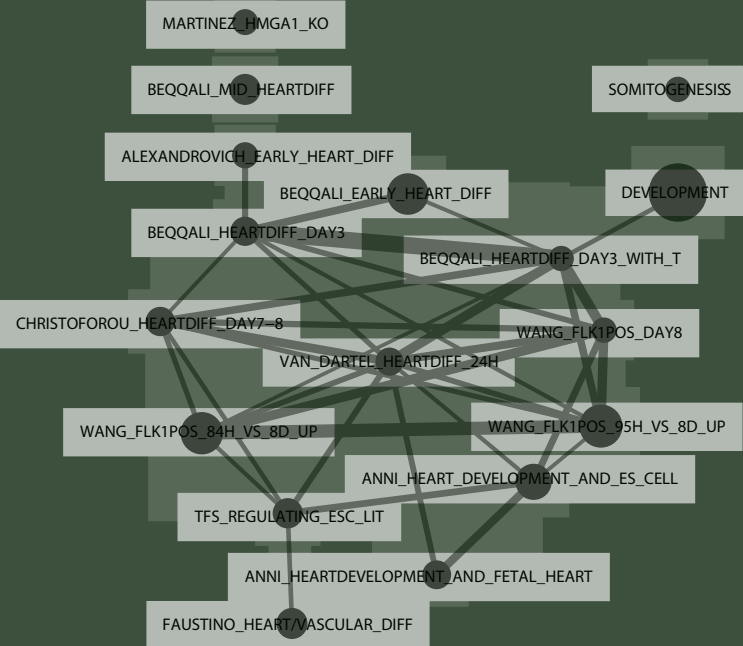

Figure 4. Molecular concept analysis of regulated gene sets in differentiation. Per set the top 20\% highest affected genes were used. The size of the gene set corresponds with the size of the node, and the boldness of the connecting lines indicates the degree of overlap between the respective gene sets. For color figure, see Color figures section.

MBP-induced gene expression changes in differentiating ESC

The gene expression profiles of the MBP exposed samples were not significantly different from their time-matched controls based on the analysis of changes in the expression of individual genes. However, an overall trend towards inhibition of differentiation by MBP seemed present as can be observed in Figure 3.

GSEA analysis showed that 30 gene sets were regulated by MBP treatment after $24 \mathrm{~h}$ of exposure (Table 2). No gene sets from C2 and Tissue Specific gene set collection were regulated. The regulated gene sets were from the $\mathrm{GO}$ gene set collection $(n=11)$ and the Cell Cycle gene set collection $(n=1)$, but most regulated gene sets were from the ESC_Cardiac gene set collection $(n=16)$. All these gene sets were up-regulated, whereas our own gene set, 'Van_Dartel_heartdiff_24h' was uniquely down-regulated. Among the regulated gene sets from the $\mathrm{GO}$ collection was the up-regulated set 'telomere_maintenance'. In addition the cell cycle related gene sets 'DNA_replication' and 'condensed_chromosome' were up-regulated, as well as 'Bar-Joseph_fibroblasts_G1_S' from Cell Cycle gene set collection. Two sets from ESC_Cardiac gene set collection, 'Behfar_pluripotent_markers' and 'Behfar_heartdiff_day7' were up-regulated after MBP exposure. Furthermore, the sets, 'Wang_Flk1_84h_vs_8d_up' and 'Wang_Flk1_95h_ vs_8d_up' were also up-regulated by MBP exposure.

These ESC_Cardiac sets have overlap in gene content as shown in Figure 5. The gene sets from the study of Zhou and co-workers showed the highest similarity. None of the 
Table 2. Regulated gene sets in monobutyl phthalate exposed ESC differentiation cultures.

\begin{tabular}{|c|c|c|c|}
\hline Gene Ontology gene set collection & \# genes/ set & NOM p-val & FDR q-val \\
\hline \multicolumn{4}{|l|}{ Upregulated } \\
\hline Dna_replication & 93 & $<1.00 \mathrm{E}-04$ & $1.36 \mathrm{E}-01$ \\
\hline Symporter_activity & 74 & $2.04 \mathrm{E}-03$ & $1.35 \mathrm{E}-01$ \\
\hline Neural_crest_cell_migration & 16 & 4.23E-03 & $1.22 \mathrm{E}-01$ \\
\hline Protein_heterodimerization_activity & 59 & 4.33E-03 & $1.28 \mathrm{E}-01$ \\
\hline Telomere_maintenance & 19 & $4.35 \mathrm{E}-03$ & $1.35 \mathrm{E}-01$ \\
\hline Response_to_unfolded_protein & 37 & $5.98 \mathrm{E}-03$ & $1.38 \mathrm{E}-01$ \\
\hline Early_endosome & 23 & $1.02 \mathrm{E}-02$ & $1.31 \mathrm{E}-01$ \\
\hline GABA-a_receptor_activity & 29 & $1.28 \mathrm{E}-02$ & $1.30 \mathrm{E}-01$ \\
\hline Protein_ubiquitination & 25 & $1.64 \mathrm{E}-02$ & 1.19E-01 \\
\hline Condensed_chromosome & 17 & $1.86 \mathrm{E}-02$ & $1.39 \mathrm{E}-01$ \\
\hline Germ_cell_development & 19 & $2.07 \mathrm{E}-02$ & $1.41 \mathrm{E}-01$ \\
\hline \multicolumn{4}{|l|}{ Cell Cycle gene set collection } \\
\hline \multicolumn{4}{|l|}{ Upregulated } \\
\hline Bar-Joseph_fibroblasts_G1_S & 133 & $1.00 \mathrm{E}-03$ & $1.34 \mathrm{E}-01$ \\
\hline \multicolumn{4}{|l|}{ Esc_Cardiac gene set collection } \\
\hline \multicolumn{4}{|l|}{ Upregulated } \\
\hline Alexandrovich_EC_cells_day4_Gata6 & 57 & 2.27E-03 & $1.32 \mathrm{E}-01$ \\
\hline Behfar_pluripotent markers & 243 & $<1.00 \mathrm{E}-04$ & $1.43 \mathrm{E}-01$ \\
\hline Behfar_heartdiff_day7 & 264 & $<1.00 \mathrm{E}-04$ & $1.25 \mathrm{E}-01$ \\
\hline Behfar_slow diff & 124 & $2.18 \mathrm{E}-03$ & 1.47E-01 \\
\hline Christoforou_low in cardiomyocytes & 67 & $4.28 \mathrm{E}-03$ & $1.34 \mathrm{E}-01$ \\
\hline Wang_Flk1Neg_95h & 107 & $<1.00 \mathrm{E}-04$ & $3.42 \mathrm{E}-02$ \\
\hline Wang_Flk1Pos_84h_vs_8d_up & 239 & $<1.00 \mathrm{E}-04$ & $1.34 \mathrm{E}-01$ \\
\hline Wang_Flk1Pos_95h_vs_8d_up & 244 & $<1.00 \mathrm{E}-04$ & $1.46 \mathrm{E}-01$ \\
\hline Wang_Flk1Pos_84h_vs_95_up & 56 & $6.48 \mathrm{E}-03$ & $1.29 \mathrm{E}-01$ \\
\hline Zhou_Oct4_Sox2_Nanog_network & 334 & $<1.00 \mathrm{E}-04$ & 1.17E-01 \\
\hline Zhou_Oct4_activation_Pou5F1 subset & 219 & $<1.00 \mathrm{E}-04$ & $8.12 \mathrm{E}-02$ \\
\hline Zhou_target_Nanog & 410 & $<1.00 \mathrm{E}-04$ & $1.38 \mathrm{E}-01$ \\
\hline Zhou_target_Oct4 & 195 & $<1.00 \mathrm{E}-04$ & $1.36 \mathrm{E}-01$ \\
\hline Zhou_Oct4_activation_5-8TF subset & 43 & $2.08 \mathrm{E}-03$ & $1.39 \mathrm{E}-01$ \\
\hline Zhou_Oct4_activation_Nanog subset & 96 & $2.20 \mathrm{E}-03$ & $1.29 \mathrm{E}-01$ \\
\hline Zhou_Oct4_activation_Esrrb subset & 60 & $8.40 \mathrm{E}-03$ & $1.09 \mathrm{E}-01$ \\
\hline Zhou_Oct4_activation_Stat3 subset & 59 & $1.02 \mathrm{E}-02$ & 1.27E-01 \\
\hline \multicolumn{4}{|l|}{ Novel gene set (this study) } \\
\hline \multicolumn{4}{|l|}{ Downregulated } \\
\hline Van_Dartel_heartdiff_24h & 42 & $<1.00 \mathrm{E}-04$ & $1.68 \mathrm{E}-03$ \\
\hline
\end{tabular}


regulated gene sets from the GO or Cell Cycle collections had overlap with any of the other regulated sets (Figure 5). Since the gene set 'Van_Dartel_heartdiff_24h' was the only gene set down-regulated by MBP treatment, there was no overlap between this gene set and the other regulated gene sets.
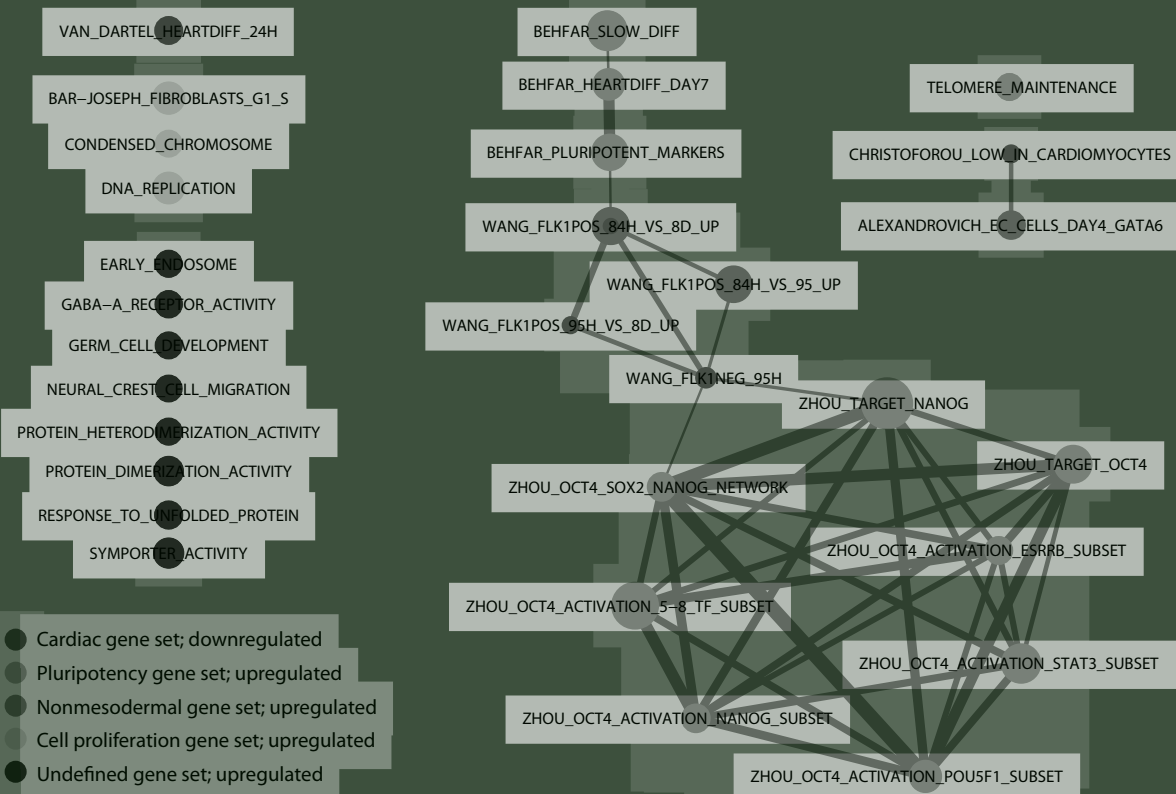

Figure 5. Molecular concept analysis of regulated gene sets in monobutyl phthalate exposed differentiation cultures. Per set the top $20 \%$ highest affected genes were used. The size of the gene set corresponds with the size of the node, and the boldness of the connecting lines indicates the degree of overlap between the respective gene sets. For color figure, see Color figures section.

\section{Discussion}

ESC differentiation and gene expression changes

Gene expression analysis of differentially expressed genes showed that based on 48 genes we could discriminate the samples isolated after 24h in EST differentiation cultures from the samples isolated earlier. This gene set showed to be enriched for 'development, 'embryonic development' and 'morphogenesis' as analyzed with GO enrichment analysis. This enrichment confirms that our gene set enables the detection of early embryonic differentiation in ESC differentiation culture within a 24 hour culture period, in the absence of cytotoxic effects. This assessment after $24 \mathrm{~h}$ exposure precludes the need for 10 day differentiation culture and subsequent time-consuming beating cardiomyocyte scoring. 
For the detection of more subtle effects at the level of gene sets we analyzed our data with GSEA [82]. This analysis works without initial filtering of the dataset to select for significantly differentially expressed genes. GSEA first ranks all genes based on fold changes in expression between two groups of samples. Subsequently, by using pre-defined sets of associated genes based on prior biological knowledge, GSEA calculates whether sets as a whole are enriched at the top or bottom of the fold change-based ranking list, or are randomly distributed. This enables the detection of significantly affected gene sets, while the fold change of expression of the individual genes can be relatively modest.

We used two collections from open sources, C2 and GO, that are commonly used for GSEA analysis in the literature. Two additional gene set collections were included, namely Tissue Specific and Cell Cycle gene set collection to allow for detection of tissue differentiation or effects on cellular proliferation. These collections were not specific for the cardiomyocyte differentiation of ESC. Therefore, we designed a fifth collection, the ESC_Cardiac gene set collection, in which gene sets derived from transcriptomic studies during cardiomyocyte or vascular differentiation of ESC were included. Using GSEA, testing these five gene set collections and the set 'Van_Dartel_heartdiff_24h', temporal changes in regulation of gene sets were revealed. None of the gene sets of the C2, Cell Cycle and Tissue Specific collections were affected by differentiation. Furthermore, only seven gene sets from the GO collection were up-regulated, of which 5 gene sets play a role in general processes, such as protein synthesis ('ribosome' and 'structural constituent of ribosome') or protein response ('response to unfolded protein'). Just 2 gene sets related to differentiation were regulated, namely 'development' and 'somitogenesis'. Up-regulation of these differentiation-related GO sets shows that using universal gene set collections it is possible to demonstrate effects on differentiation. However, these collections contain few gene sets for specific differentiation routes. Studying effects on specific differentiation processes using universal gene set collections does not give insight into the specific niche of our interest. For this reason these gene set collections are not the most suitable ones for studying cardiomyocyte differentiation-related processes.

GSEA with the ESC_Cardiac gene set collections showed regulation of 16 gene sets. This indicates that dedicated gene sets allow for detection of cardiomyocyte differentiation related effects. The majority of these gene sets were up-regulated and are related to heart differentiation. The gene sets 'Anni_heart_development_and_ES_cell' and 'Anni_ heart_development_and_fetal_heart' were made using the Anni 2.0 text-mining tool [138]. These gene sets comprise genes which are most significantly co-published with the concept 'heart development' and respectively 'ESC and 'fetal heart'. The up-regulation of these text-mining based heart development-related gene sets indicate that the ESC differentiate towards cardiomyocytes. Beqqali et al. studied cardiomyocyte differentiation of human ESC. They identified and validated time-dependent gene expression patterns [28]. The gene set'Beqqali_early_heartdiff' contains genes up-regulated in day 3 isolated 
differentiation cultures compared to the reference pool, which contains samples of day $1,3,6,9$ and 12 cardiomyocyte differentiation and human fetal heart as positive control. The gene sets 'Beqqali_heartdiff_day3_with_T' and 'Beqqali_heartdiff_day3' comprises genes which are up-regulated after 3 days of differentiation culture, respectively with and without co-expression of the mesodermal marker $\mathrm{T}$, compared to the reference pool. These three gene sets are characteristic for the first phase of cardiomyocyte differentiation, which is similar to the differentiation period we describe in this study.

The up-regulation of the sets 'Wang_Flk1pos_day8', 'Wang_Flk1pos_84h_vs_8d_up' and 'Wang_Flk1pos_95h_vs_8d_up' in the control samples isolated after $24 \mathrm{~h}$ is caused by the genes associated with the cardiomyocyte lineage present in these sets. The strongest regulated genes in this set by cardiomyocyte differentiation are the mesodermal markers T and Mesp1, furthermore other mesodermal and cardiac differentiation related genes contributed to the significance of these gene sets.

As shown in the molecular concept analysis (Figure 4), there is a high overlap between the gene sets related to cardiac differentiation. Because our gene set 'Van_Dartel_heartdiff_24h' includes genes that mark early cardiomyocyte differentiation, it has a high overlap with other gene sets characteristic for cardiac differentiation.

Besides up-regulation of cardiac differentiation-related gene sets, two gene sets were down-regulated. These gene sets include genes characteristic for the pluripotent state of stem cells. The down-regulation of the pluripotency-associated gene sets after $24 \mathrm{~h}$ of suspension culture indicates loss of the pluripotent state of the cell because of initiation of cardiomyocyte differentiation. The down-regulated set 'Behfar_pluripotent_markers' contains genes which were down-regulated after induction of differentiation by adding a differentiation inducing cocktail compared to cultured cells without differentiation induction. The down-regulated set 'Behfar_heartdiff_day7' contains genes which are down-regulated at day 7 of cardiomyocyte differentiation compared with day 12 [132]. Most likely, these genes were first down-regulated when differentiation was initiated, and become up-regulated at the latest phase of the differentiation protocol. This effect is also shown in the transcriptomic study of Bruce et al.[29]. They showed a large group (>1000 genes) of stem cell genes to be down-regulated from day 3 onwards, when the cells loose their pluripotency, and become up-regulated again from day 12 onward. Nanog, one of the transcription factors of the pluripotent marker trio Pou5f1Sox2-Nanog, was significantly down-regulated after initiation of differentiation and was up-regulated again from day 6 onwards [29]. Nanog is also present in the 'Behfar_heartdiff_day7' gene set. Furthermore the genes in this gene set showed overlap with the 'Behfar_pluripotent_markers' gene set, which indicates that the 'Behfar_heartdiff_day7' gene set contains genes which are normally highly expressed in pluripotent stem cells.

In summary, GSEA shows temporal up-regulation of gene sets related to cardiac differentiation, including our novel gene set 'Van_Dartel_heartdiff_24h', whereas gene sets 
related to pluripotency are down-regulated. These findings are mechanistically concordant and indicate that early cardiac differentiation of ESC in culture can effectively be monitored within the $24 \mathrm{~h}$ period studied.

MBP related gene expression changes in differentiating ESC

At the individual gene level, no significant MBP-related gene expression changes were found. This is most likely due to the presence of different cell types in the EB, which considerably decreases the power of the analysis. Seiler et al. observed that at day 7 of the differentiation protocol the highest percentage of cardiomyocytes was present in the culture, namely $17 \%$ [25]. Likewise, in our study the detection of subtle changes on the level of single gene expression specific for such a small subset of cells appeared difficult. However, an overall trend towards inhibition of differentiation by MBP seemed present (Figure 3). For detection of subtle gene expression changes induced by MBP we performed GSEA pathway level analysis using five gene set collections as well as the set 'Van_Dartel_heartdiff_24h'. No significantly regulated gene sets were found in the C2 and Tissue Specific gene set collection. In the GO gene set collection, 11 gene sets were found to be regulated as a consequence of a 24h MBP treatment of EB in suspension culture. Regulation of most gene sets could not be explained from a biological point of view by inhibition of cardiomyocyte differentiation by MBP. However, the up-regulation of the set 'telomere_maintenance' is indicative of inhibition of early cardiomyocyte differentiation, because normally telomerase activity becomes down-regulated during differentiation [139]. The higher expression of this gene set in the MBP-treated samples points towards inhibition of differentiation. Furthermore, two gene sets, 'DNA_replication' and 'condensed_chromosome', were up-regulated. This up-regulation implies an increased proliferation rate in MBP exposed samples. Doss et al. previously showed down-regulation of proliferation-related gene sets, under which these two sets, in 15day old cardiomyocyte differentiation cultures compared with undifferentiated ESC [26]. From this study it remains unclear in which phase of differentiation the proliferation rate decreases. It was shown recently that down-regulation of Nanog, which occurs when cells start to differentiate, impaired cell proliferation [140]. Since we know from literature that Nanog is repressed from initiation of differentiation onwards [29], we expect the cells to mainly proliferate in the early phase of the differentiation protocol, and that the proliferation rate decreases when the cells become more specialized. Additionally, the Cell Cycle set, 'Bar-Joseph_fibroblasts_G1_S', was up-regulated in these samples, which also points towards an increased proliferation rate in these MBP exposed samples. A higher proliferation rate in the MBP exposed differentiation culture is therefore an indication for inhibition of differentiation. 
The gene sets 'Behfar_pluripotent_markers' and 'Behfar_heartdiff_day7' were upregulated by MBP exposure. As discussed, these sets were down-regulated during differentiation and contained pluripotent-associated genes.

The molecular concept analysis shows the high overlap between the different gene sets originally described in the study of Zhou et al. [131]. These gene sets contain genes that are targets of specific transcription factors known to play a role in the molecular circuitry of pluripotency. These transcription factors do not necessarily up-regulate pluripotency associated genes, but can also inactivate or repress differentiation related genes. From literature it is known that genes that are activated by a combination of transcription factors present in ESC are expressed in the pluripotent state and become repressed upon differentiation [141]. The high interaction of the regulated gene sets of Zhou et al. indicates that these transcription factors coregulate the expression of these target genes. This means that the top $20 \%$ of the regulated genes in these sets are expected to be expressed in pluripotent cells. The higher expression of these pluripotency associated gene sets from the study of Zhou et al. in the MBP-exposed samples confirms the inhibition of differentiation by the treatment.

Besides the higher pluripotent state of the MBP treated cells, also more nonmesodermal differentiation was observed. The genes in the regulated gene sets that are derived from the study of Wang et al. are genes which are higher expressed in Flk1+ cells after both 84 and $95 \mathrm{~h}$ of differentiation than in Flk1- cells. Flk1+ cells were used for endothelial differentiation in the study of Wang et al., however these Flk1+ cells can also differentiate towards the hematopoietic and cardiac lineage [136]. The genes in these sets contain genes specific for endothelial, hematopoietic and cardiac differentiation, but furthermore genes not specific for mesodermal differentiation were present in these sets. The up-regulation of these gene sets as a consequence of MBP treatment, is explained by the higher expression of nonmesodermal Flk1+ related genes. Up-regulation of nonmesodermal genes in MBP exposed samples also causes up-regulation of the gene sets 'Christoforou_low_in cardiomyocytes' and 'Alexandrovich_EC_cells_day4_Gata6'.

The inhibition of cardiomyocyte differentiation by MBP-exposure was most obvious through the down-regulation of our novel gene set, 'Van_Dartel_heartdiff_24h', which specifically describes the niche of early cardiomyocyte differentiation. This cardiomyocyte differentiation related gene set was down-regulated after MBP exposure, with a GSEA calculated $p$-value of $<0.001$ and an FDR of 0.002 .

In summary, the MBP induced inhibition of beating cardiomyocytes scored at day 10 of culture was parallelled by gene expression changes observed as early as $24 \mathrm{~h}$ after exposure onset. These changes included increased expression of pluripotency-, proliferation- and nonmesodermal differentiation related gene sets and most significantly, the unique down-regulation of our novel gene set identified in this study, which specifically 


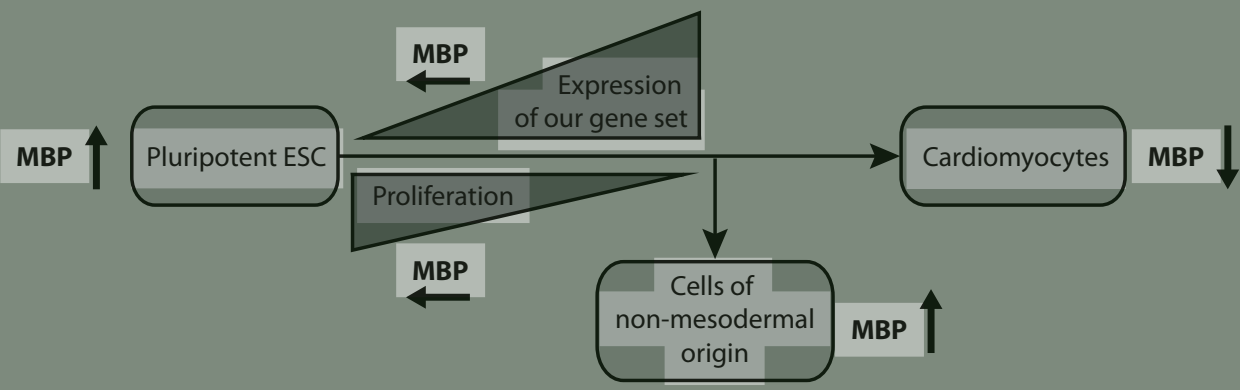

Figure 6. Summary scheme of monobutyl phthalate regulated inhibition (MBP) of ESC cardiomyocyte differentiation as observed in this study.

describes the niche of early cardiomyocyte differentiation. The combination of these findings show a consistent pattern of cardiomyocyte differentiation inhibition (Figure 6).

We showed that, besides our novel gene set 'Van_Dartel_heartdiff 24h', other gene sets showed temporal regulation (Table 1) functionally related to cardiomyocyte differentiation. However, these sets were not down-regulated after MBP exposure, in spite of the overlap of genes present in these sets with the 'Van_Dartel_heartdiff_24h' gene set (Figure4). Our novel identified gene set contained well known genes involved in mesodermal and early cardiomyocyte differentiation, such as T, Fgf8, Mesp1 and Mix/1. These genes were only present in some of the gene sets that had significant overlap with 'Van_Dartel_heartdiff_24h'. In addition, the set 'Van_Dartel_heartdiff_24h' contained several genes involved in mesodermal and early cardiomyocyte differentiation that were not present in any of the temporally regulated gene sets that showed overlap with 'Van_Dartel_heartdiff_24h'. Furthermore, among the MBP-regulated genes of the set 'Van_Dartel_heartdiff_24h', genes are present which are related to inhibition of cell proliferation. The unique combination of the genes present in the 'Van_Dartel_heartdiff_24h' gene set was essential for identification of cardiomyocyte inhibition by MBP at this early stage of differentiation.

In conclusion, we demonstrated cardiomyocyte differentiation of ESC at the level of gene expression at an early stage in the differentiation process. With GSEA, using gene sets that specifically apply to the system studied, including our novel gene set'Van_Dartel_heartdiff_24h', the sensitivity of the analysis was greatly improved. MBP induced changes in gene expression profile were demonstrated mainly as an up-regulation of gene sets after $24 \mathrm{~h}$ of suspension culture relative to the nonexposed timed control. These gene sets were related to pluripotency, cell proliferation and nonmesodermal differentiation, respectively. Direct evidence for inhibition of cardiomyocyte differentiation was given by the down-regulation of (mesoderm-derived) cardiac differentiation genes observed with our gene set ('Van_Dartel_heartdiff_24h'). It is known from in vivo studies that MBP causes mainly malformations of tissues of mesodermal origin [91-93, 122]. 
MBP induced inhibition of mesoderm-derived cardiac differentiation as demonstrated on the gene expression level is therefore in line with in vivo embryotoxicity. The gene set defined in this study might serve as a starting point for defining a dedicated gene set for detecting embryotoxicity in the EST. More specifically, comparison with other phthalates could lead to a gene set for the prediction of embryotoxicity of the chemical class of phthalates. In addition, these findings offer possibilities for further application of the transcriptomic approach to define the applicability domain and the predictivity of the EST.

\section{Acknowledgements}

This study was supported by grant MFA6809 from the Dutch technology society foundation STW. The authors thank Abdelaziz Beqqali (Hubrecht Laboratory, Utrecht, The Netherlands) for providing the microarray results of their experiment. 

Dorien A.M. van Dartel, Jeroen L.A. Pennings,

Frederik J. van Schooten, Aldert H. Piersma

Toxicology and Applied Pharmacology (2010) 243:420-8 
CHAPTER 4

Transcriptomics-based identification of developmental toxicants through their interference with cardiomyocyte differentiation of embryonic stem cells 


\begin{abstract}
The Embryonic Stem cell Test (EST) predicts developmental toxicity based on the inhibition of cardiomyocyte differentiation of embryonic stem cells (ESC). The subjective end point, the long culture duration together with the undefined applicability domain and related predictivity need further improvement to facilitate implementation of the EST into regulatory strategies. These aspects may be improved by studying gene expression changes in the ESC differentiation cultures and their modulation by compound exposure using transcriptomics. Here, we tested the developmental toxicants monobutyl phthalate and 6-aminonicotinamide. ESC were allowed to differentiated, and cardiomyocyte differentiation was assessed after 10 days of culture. RNA of solvent controls was collected after $0,24,48,72$ and $96 \mathrm{~h}$ of exposure, and RNA of developmental-toxicantexposed cultures was collected after 24 and $96 \mathrm{~h}$. Samples were hybridized to DNA microarrays, and 1355 genes were found differentially expressed among the unexposed experimental groups. These regulated genes were involved in differentiation-related processes, and Principal Component Analysis (PCA) based on these genes showed that the unexposed experimental groups appeared in chronological order in the PCA, which can therefore be regarded as a continuous representation of the differentiation track. The developmental-toxicant-exposed cultures appeared to deviate significantly from this differentiation track, which confirms the compound-modulating effects on the differentiation process. The incorporation of transcriptomics in the EST is expected to provide a more informative and improved end point in the EST as compared with morphology, allowing early detection of differentiation modulation. Furthermore, this approach may improve the definition of the applicability domain and predictivity of the EST.
\end{abstract}




\section{Introduction}

Alternative methods for hazard identification in developmental toxicology have been the subject of extensive research over the last three decades. This research has been driven partly by the relatively high animal use in developmental toxicity testing and the call for reduction, replacement and refinement of animal testing. This has stimulated the design of alternative methods that could predict developmental toxicity at an early stage of compound development while requiring only a small volume of compound and a minimal number of experimental animals. The designed alternative tests vary from whole embryo cultures of rat or zebrafish, and organ cultures to continuous cell-linebased tests [19, 84-86].

Cell-line-based tests are completely animal-free, and include the Embryonic Stem cell Test (EST), which was validated by the European Centre for the Validation of Alternative Methods (ECVAM) [19]. The EST uses a continuous murine embryonic stem cell (ESC) line, which can be induced to differentiate into contracting cardiomyocytes, via the formation of embryoid bodies (EB). Within EB, cells of ecto-, meso-, and endodermal origin develop upon continued in vitro culture $[6,87]$. This resembles the in vivo situation in which the mesodermal germ layer develops between the ectoderm and endoderm germ layers [113], and stipulates that cell-cell interactions may play a crucial role in the formation of cardiac cells in the EST.

In the EST protocol the extent of differentiation is evaluated microscopically after 10 days of culture. Compound induced inhibition of cardiomyocyte differentiation combined with cytotoxicity data is then used for the prediction of embryotoxicity. Disadvantages of this protocol are the subjective scoring of contracting cardiomyocyte outgrowths, the duration of culture and the limited basis of the existing prediction model in terms of compounds tested [22]. In addition, the current model misses the opportunity to study effects on different developmental processes which accompany and may be mediating the development of beating cardiomyocytes. Information about these processes could help to define the applicability domain of the EST and could lead to improved predictivity.

A series of studies have revealed important developmental pathways, which are activated during different phases of cardiomyocyte differentiation [26-28, 111, 132-135, 142-145]. However, most of these studies describe genes involved in late stages of differentiation. Recently, we identified a set of genes ('Van_Dartel_heartdiff_24h') that was regulated during the first $24 \mathrm{~h}$ of cardiomyocyte differentiation [111]. We showed that this gene set was down-regulated when differentiation cultures were exposed to the developmental toxicant monobutyl phthalate (MBP). Chemical embryotoxicity in ESC differentiation cultures monitored by gene expression have been studied before, but only with small selected groups of genes [30, 31]. 
The application of gene expression analysis to detect effects on the differentiation process has shown promising results $[111,120,146]$ and could be used to improve the model in each of the aspects mentioned above. In the present study, differentiation cultures were exposed to MBP or 6-aminonicotinamide (6-AN). Both compounds mainly affect structures of mesodermal origin. For MBP these effects include cleft palate, cardiovascular, urogenital, and skeletal malformations [91-93, 122], and 6-AN has been shown to induce cleft palate and skeletal malformations [147-149]. Given that 6-AN is a much more potent developmental toxicant than MBP and likely induces malformations via a different mechanism, it is interesting to study if inhibition of cardiomyocyte differentiation in the EST by both compounds can be detected using a common set of genes.

Cardiomyocyte differentiation of ESC was induced through specific culture conditions, based on the ECVAM EST protocol [7]. RNA from control cultures was isolated after $0,24,48,72$ and $96 \mathrm{~h}$ from the EB-stage onward. We identified 1355 genes that were significantly regulated among the time points, which collectively describe the differentiation track' in a Principal Component Analysis (PCA). Differentiation cultures exposed to MBP or 6-AN from the EB-stage onward for 24 or $96 \mathrm{~h}$ showed RNA expression patterns statistically significantly deviating from the differentiation track. We show that the identified gene set, which is regulated during ESC differentiation, can be used to detect differentiation-disrupting effects of the two diverse developmental toxicants tested. The implications for further development of the assay are discussed.

\section{Materials and methods}

Pluripotent embryonic stem cell culture

Pluripotent murine D3 embryonic stem cells (ESC; ATCC, Rockville, MD) were routinely subcultured every 2-3 days and grown as a monolayer in complete medium, consisting of Dulbecco's Modified Eagle Medium (Gibco BRL, Gaithersburg, MD) supplemented with $20 \%$ heat-inactivated fetal bovine serum (Hyclone, Logan, UT), 1\% non-essential amino acids (Gibco BRL, Gaithersburg, MD), 1\% penicillin/ streptomycin (Gibco BRL, Gaithersburg, MD), 2 mM L-glutamine (Gibco BRL, Gaithersburg, MD) and $0.1 \mathrm{mM}$ $\beta$-mercapto-ethanol (Sigma-Aldrich, Zwijndrecht, The Netherlands). Leukemia inhibitory factor (LIF; Chemicon, Temecula, CA) was added directly to the culture disk in a final concentration of 1000 units $/ \mathrm{ml}$. The cells were maintained in a humidified atmosphere at $37^{\circ} \mathrm{C}$ and $5 \% \mathrm{CO}_{2}$.

Cardiomyocyte differentiation culture

Embryoid body (EB) formation was used as the initial step for ESC differentiation cultures (Figure 1). EB were obtained via hanging drop culture in complete medium without 
Day 0
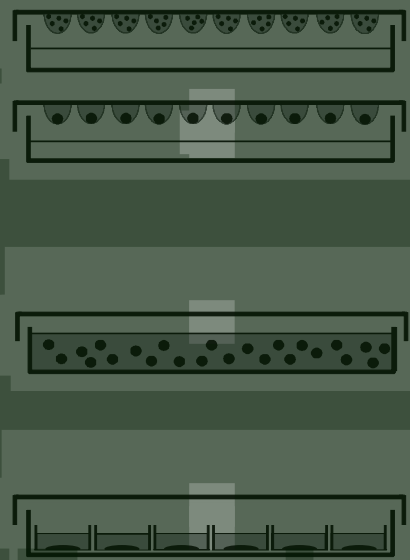

Day 10

Evaluation contracting cardiac muscle foci

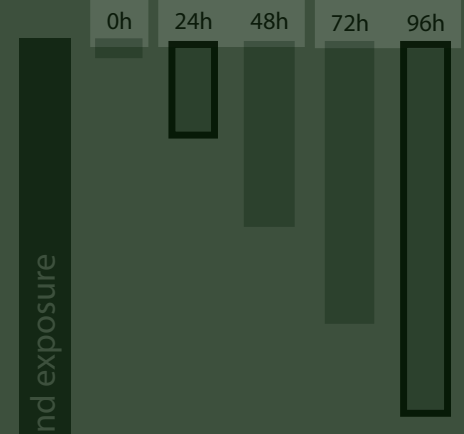

Sampling solvent controls

Sampling exposed cells

Figure 1. Hanging drops, containing single cell suspensions, were set up at day 0 . The single cells formed embryoid bodies (EB), which were transferred to suspension culture at day 3. At day 5, one EB was plated per well in a 24 wells plate. At day 10, each EB was microscopically evaluated for the presence of contracting foci. Cultures were exposed from day 3 onwards to monobutyl phthalate (MBP) or 6-aminonicotinamide (6-AN). RNA was collected at selected exposure durations as indicated.

LIF as described earlier [7, 102]. In brief, stem cell suspensions $\left(3.75 \cdot 10^{4}\right.$ cells $\left./ \mathrm{ml}\right)$ were placed on ice before the set up of the culture. Drops $(20 \mu \mathrm{l})$ were placed onto the inner side of the lid of a Petri dish filled with phosphate buffered saline (PBS; Gibco BRL, Gaithersburg, MD) and incubated in a humidified atmosphere at $37^{\circ} \mathrm{C}$ and $5 \% \mathrm{CO}_{2}$. After 3 days of hanging drop culture, EB had formed and these were subsequently transferred to bacterial Petri dishes (Greiner Bio-one, Frickenhausen, Germany). On day 5, EB were plated one per well into 24-well tissue culture plates (TPP, Trasadingen, Switzerland). Differentiation was determined by microscopical inspection of EB outgrowths into 
contracting myocardial cells after 10 days of culture. EB were considered cardiomyocyte positive if at least one contracting focus was present.

Test compounds and inhibition of cardiomyocyte differentiation

ESC differentiation cultures were exposed from the EB stage at day 3 onwards to monobutyl phthalate (MBP; TCI Europe, Zwijndrecht, Belgium; CAS No. 131-70-4), 6-aminonicotinamide (6-AN; Fluka, Buchs, Switzerland; CAS No. 329-89-5) or vehicle $(0.20 \%$ dimethyl sulfoxide (DMSO)). MBP and 6-AN were tested at their $\mathrm{ID}_{50}$ concentration, the concentration at which the number of EB containing contracting foci was reduced to $50 \%$ of the control. We determined these noncytotoxic $I D_{50}$ concentrations previously [150], and tested MBP at $1.4 \mathrm{mM}$, and 6-AN at $4.4 \mu \mathrm{M}$.

To confirm the $\mathrm{ID}_{50}$ of MBP and 6-AN, parallel exposed cultures were microscopically evaluated at day 10 of the culture and others were used for gene expression analysis at various time points as described below.

RNA isolation and gene expression profiling

MBP and 6-AN exposed cultures were sampled after 24h (culture day 4) and 96h (culture day 7) after the start of exposure. Vehicle control differentiation cultures were harvested after 0, 24, 48, 72 and 96h (culture days 3, 4, 5, 6 and 7) from the EB-stage at day 3 (Figure 1). Cells were directly collected in RNA protect (Qiagen, Venlo, The Netherlands) to stabilize RNA, and total RNA was purified using the RNeasy mini kit (Qiagen, Venlo, The Netherlands) with an additional DNase treatment (RNase-Free DNase Set; Qiagen, Venlo, The Netherlands) according to the manufacturer's instructions. The quantity of RNA in each sample was measured using a NanoDrop Spectrophotometer (NanoDrop technologies, Wilmington, DE). RNA integrity was assessed on the 2100 Bioanalyzer (Agilent Technologies, Amstelveen, The Netherlands), and good quality RNA was used for gene expression analysis (RNA Integrity Number (RIN) >8).

Microarray analysis experiments were performed as described previously with minor changes [75, 111]. In brief, as it was not practically feasible to process all samples simultaneously, RNA samples were randomized and processed over several experimental days, using one RNA sample per array and with a mix of all samples as common reference. All samples were run within a 2-week time period. Of the RNA samples (7-8 independent biological replicates per group), $1.5 \mu \mathrm{g}$ was amplified using the Amino Allyl MessageAmp II aRNA kit (Ambion, Austin, TX). Next, $10 \mu \mathrm{g}$ of control or exposed sample aRNA was labeled with Cy3, and equal amounts of reference pool with Cy5 (CyDye Post Labeling Reactive Dye Pack; Amersham Biosciences, Buckinghamshire, UK). The labeled and purified aRNA samples were fragmented and combined with reference pool. The samples were hybridized to microarray slides which were spotted at the Microarray Department of the University of Amsterdam. The slides contain 21,997 65-mer oligonucleotides from 
the Sigma-Compugen Mouse nucleotide library, as well as positive and negative controls. The microarray slides were scanned using a ScanArray 4000XL microarray scanner (Perkin-Elmer, Waltham, MA) with appropriate laser and photomultiplier tube settings. Array Vision software (Imaging Research, St. Catherines, Ontario, Canada) was used to determine median Cy3 and Cy5 signal intensities for each separate spot and background signal. Quality control was performed on raw data by means of a scatter plot and MAplot as well as a normal probability plot to assess signal distribution. The positive and negative controls present on the slide were used for quality control, but excluded from further analysis.

Data analysis and statistics

Data normalisation and statistical analysis were carried out using the R statistical software environment [151]. Raw microarray signal data were normalized using a three-step approach as described previously [123]. For each gene maximal fold ratios (FR) in gene expression between the experimental groups were determined by comparing the average normalized signal values per group and were calculated as the maximum/minimum ratio. Genes that were differentially expressed between any of the experimental groups were identified by a one-way ANOVA on the normalized data. A p-significance threshold of 0.001 and FR>1.5 was used to select differentially expressed genes. Arrangement of differentially expressed genes in expression group clusters was achieved by hierarchical clustering based on Euclidian distance and Ward linkage, using GeneMaths (Applied Maths, Sint-Martens-Latem, Belgium).

To explore matching concept profiles of these significantly regulated gene clusters with concept profiles of biological processes in the Gene Ontology Database, we used the text-mining tool Anni 2.0 [138]. This tool calculates the overall matching score, the cohesion score, between each gene of the gene cluster with the concept profiles of GO biological processes. The concept with the highest sum of cohesion scores is defined as the predominant gene cluster function.

Analysis of gene set regulation using GSEA

Gene Set Enrichment Analysis (GSEA) was performed to detect differential expression of biologically relevant sets of genes that share common biological function or regulation $[82,152]$. For the GSEA analysis, we used the default parameters and 1000 permutations. Gene sets were considered being significantly affected when the $p$-value was $<0.01$ and the False Discover Rate (FDR) $<0.05$. GSEA was followed by molecular concept analysis, in which overlap of genes between gene are visualized within a network [125]. The overlap between the gene sets was calculated as we described previously [111]

For the GSEA we used the gene set collection, named ESC_Diff, which we created ourselves. As basis for this gene set collection we used the gene set collection ESC_Car- 
diac, described earlier [111], which contains 103 gene sets. We extended this gene set collection with 145 gene sets involved in differentiation presented in the literature. Gene sets were included using data on ESC cardiomyocyte differentiation and cardiac development [26, 27, 133, 142-145]. Also gene sets specific for different stages of embryonic development and stem cells were included [153]. Furthermore, gene sets that are related to mesodermal differentiation were included. These sets were derived from a transcriptomic study in which the expression profile of $B M P 2^{+}$EB was compared with control EB and ESC [154]. Furthermore, gene sets were included from studies in which target genes of pluripotency-related transcription factors, such as Nanog, Pou5f1 and Sox2, were identified [140, 141, 155-157]. More gene sets related to the pluripotent cell state were included from a meta-analysis study [158] and a common expression analysis of 6 ESC lines, and of 5 ESC and 2 embryonal germ cell lines [159, 160]. Gene sets involved in pluripotency and differentiation were obtained from studies in which gene expression patterns of ESC were compared with DMSO and retinoic acid-induced differentiated ESC and differentiation of ESC by LIF-removal [161-164]. Also gene sets related to ESC differentiation derived from literature using the test-mining tool Anni 2.0 were included [138]. In addition, also gene sets were included from transcriptomic studies that describe non-cardiomyocyte differentiation, including neural, pancreatic and renal differentiation $[29,165,166]$.

Definition of the differentiation track

To visualize the differences between the experimental groups, we applied a novel approach based on Principal Component Analysis (PCA) [58]. PCA is a mathematical algorithm that describes the data on the basis of their (dis)similarity, so that a greater distance corresponds with a greater dissimilarity. In this analysis, a principal component is defined as a mathematically derived combination of genes and their expression characteristics that can be used to describe part of the process observed. A number of principal components that are mutually independent can be derived which in combination describe the process under study. Here, we first performed PCA using the genes that were identified to be differentially expressed when ESC differentiate. Secondly, the different time points of the differentiation process in culture could be connected with a curve in the PCA-plot obtained, which is therefore a continuous representation of ESC differentiation based on the dynamics of gene expression. This representation will be further referred to as the 'differentiation track'.

Coordinates along the first and second principal component, after PCA transformation, were calculated for each sample. Deviation of compound exposed cultures from the PCA-derived 'differentiation track' was analyzed by a $t$-test on the first and second principal components coordinates compared to these of the time-matched control cultures. Cultures were considered to deviate statistically significant from the track, if $p<0.05$. 


\section{Results}

Inhibition of cardiomyocyte differentiation

The presence of contracting cardiomyocyte foci in EB after exposure to either MBP or 6-AN at their $\mathrm{ID}_{50}$ was assessed at day 10 of the culture. We confirmed the ID50 concentration of MBP and 6-AN as previously determined [150]. Exposure showed that the number of MBP or 6-AN exposed EB containing contracting cardiomyocytes was decreased with $44 \%$ and $40 \%$, respectively, compared to control (data not shown). Parallel cultures were used for RNA transcript analysis, as described below.

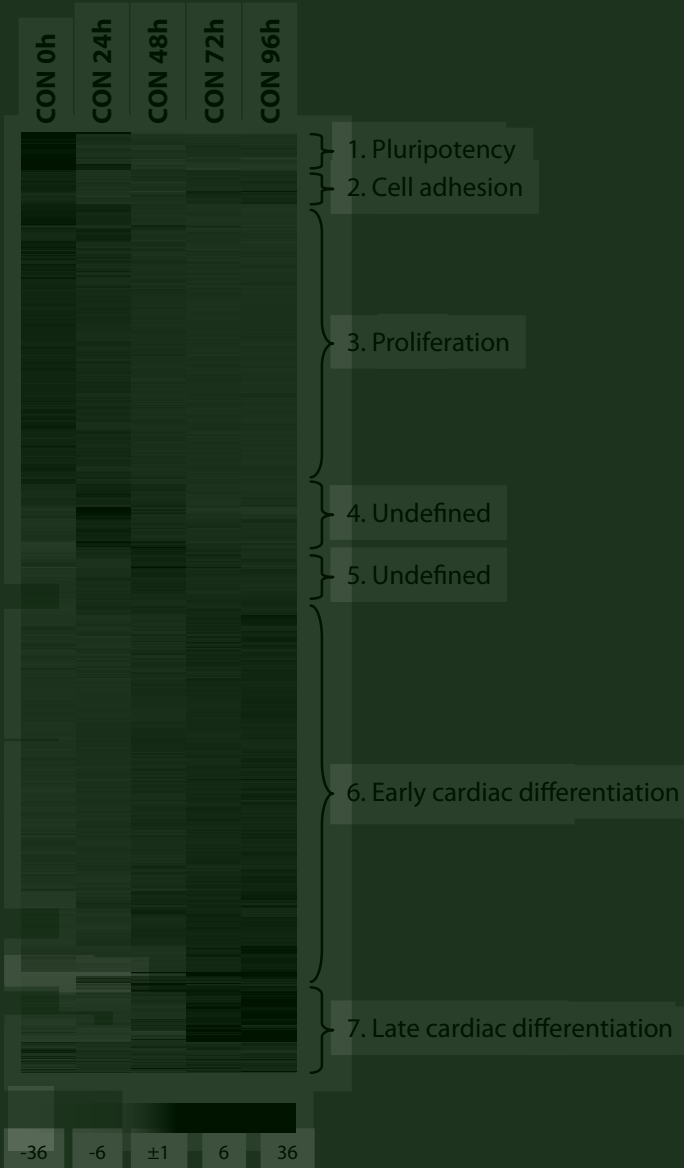

Figure 2. Heatmap of all 1355 significantly regulated genes (fold ratio $>1.5$ and $p<0.001$ ) transcriptionally responsive to cardiac differentiation. Each row represents the relative expression of a single gene throughout the differentiation. Seven clusters are discriminated based on temporal gene expression of these regulated genes. The predominant function of each cluster is given. For color figure, see Color figures section. 
ESC differentiation related gene expression changes

Gene expression data of control samples were compared by a one-way ANOVA. Using the cut-off criteria of a $p$-value of $<0.001$ and a FR>1.5, 1355 regulated genes were differentially expressed among the chemically unexposed experimental groups. The clustering of these genes is presented in Figure 2. Broadly seven clusters of gene expression profile can be distinguished on the basis of their expression dynamics with time (Figure 2, clusters 1-7 and Supplemental Table 1).

Table 1. Significance of deviation from the 'differentiation track' on the first (PC1) and second (PC2) principal component of cultures exposed for 24 or $96 \mathrm{~h}$ to monobutyl phthalate (MBP) or 6-aminonicotinamide (6-AN). ${ }^{*} p<0.05$.

\begin{tabular}{cccc}
\hline Compound & Exposure duration & $\boldsymbol{p}$-value PC 1 & $\boldsymbol{p}$-value PC 2 \\
\hline MBP & $24 \mathrm{~h}$ & $0.041^{*}$ & 0.096 \\
MBP & $96 \mathrm{~h}$ & 0.059 & 0.493 \\
6-AN & $24 \mathrm{~h}$ & 0.164 & $0.031^{*}$ \\
6-AN & $96 \mathrm{~h}$ & 0.587 & $0.017^{*}$ \\
\hline
\end{tabular}

The predominant gene cluster functions of the seven clusters as determined by Anni 2.0 text-mining analysis are presented in Figure 2. For two gene clusters, cluster 4 and 5 , no predominant gene cluster function could be identified. For cluster 4, several concepts with equal sums of the cohesion scores were identified, while for cluster 5 the sum of the cohesion scores relative to the number of genes present in this cluster was low (< $0.0065)$, this indicates that no enriched biological process could be identified.

GSEA was performed to further study the progress of differentiation towards the time points at which exposed cultures were sampled ( 24 and 96 h). After $24 \mathrm{~h}$ of suspension culture, 51 gene sets were up-regulated and 91 gene sets were down-regulated compared with the samples isolated after Oh of suspension culture (Supplemental Table 2). The significance of overlap among up-regulated gene sets in gene content, shown as connections, is visualized in Figure 3. All up-regulated gene sets were connected in one network. These gene sets are enriched in genes involved in differentiation processes, including cardiac differentiation, and includes cluster 4 ('Cl4_24h_up'). Of the downregulated gene sets, 87/91 were connected in one network. These sets comprise genes which are up-regulated in pluripotent ESC and at the early stage of ESC differentiation, including the gene set 'Van_Dartel_heartdiff_24h' (Figure 3A).

GSEA of gene sets regulated between $96 \mathrm{~h}$ and $0 \mathrm{~h}$ in unexposed cultures revealed upregulation of 72 gene sets and down-regulation of 73 gene sets. Of all up-regulated gene sets, 71/72 have significant overlap in gene content with at least one other up-regulated gene set (data not shown). These gene sets are characteristic for differentiation, and in particular cardiac differentiation. Most of these gene sets were already up-regulated after $24 \mathrm{~h}$ 


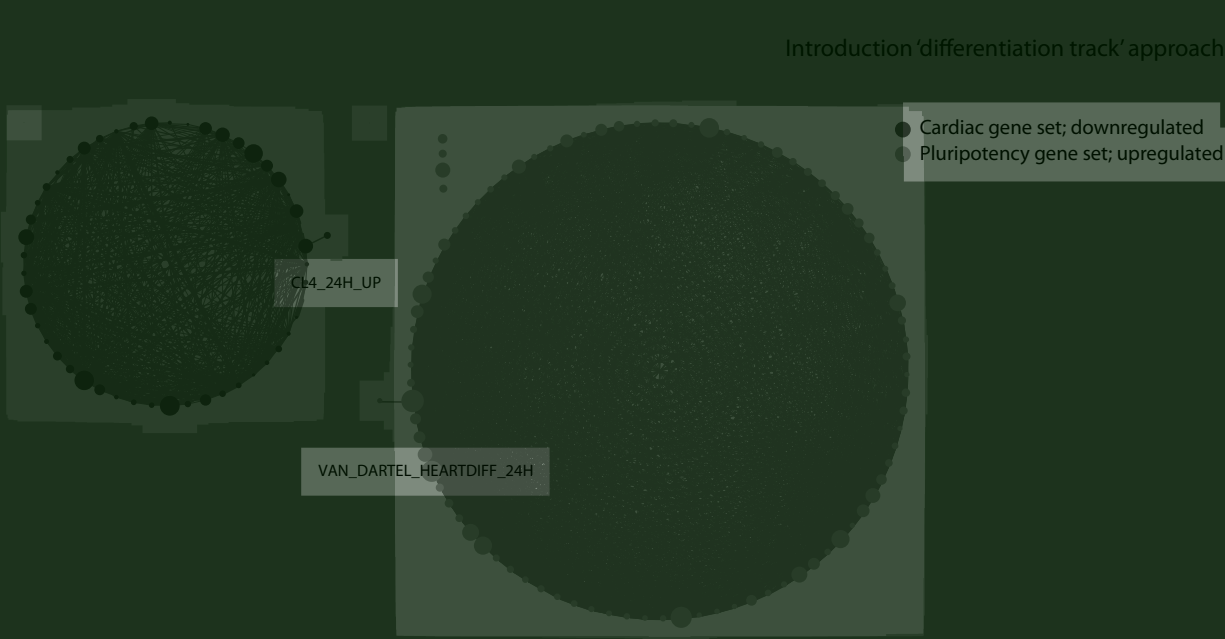

Figure 3. Molecular concept analysis of regulated gene sets after $24 \mathrm{~h}$ of differentiation. Per set the top $20 \%$ highest affected genes were used. The size of the gene set corresponds with the size of the node, and the width of the connecting lines indicates the degree of overlap between the respective gene sets. For color figure, see Color figures section.

(45/72). The majority of the additional up-regulated gene sets are described to be related to later stages of cardiomyocyte differentiation. All down-regulated gene sets showed significant overlap in genes responsible for the significance of the gene set regulation, and together form one network (data not shown). These gene sets describe pluripotency and early differentiation stages. A majority of these gene sets were already significantly down-regulated after $24 \mathrm{~h}(68 / 73)$. The additional down-regulated gene sets include sets comprising genes described to be down-regulated at later stages of differentiation, a gene set containing targets of transcription factors that maintain pluripotency, and another pluripotency-related set. In general, these GSEA results show that as early as $24 \mathrm{~h}$ after plating EB, ESC differentiation can be detected by up-regulation of differentiation gene sets and down-regulation of pluripotency-related gene sets. The $96-\mathrm{h}$ time point only adds the up-regulation of gene sets describing later stages of cardiomyocyte differentiation.

\section{ESC cardiac differentiation track}

Principal Component Analysis (PCA), on the basis of all 1355 significantly regulated genes, showed that $93.8 \%$ of all variance between experimental groups could be described using the first and second principal components of the PCA analysis. The remaining principal components had minor contributions to total gene expression variance and produced no significant shifts between experimental groups. The two-dimensional PCA-plot showed that replicates within different experimental groups clustered together and different groups appeared in chronological order in the PCA (Figure 4). The arc connecting these experimental groups can therefore be regarded as a representative description of the 'differentiation track' by gene expression changes. 


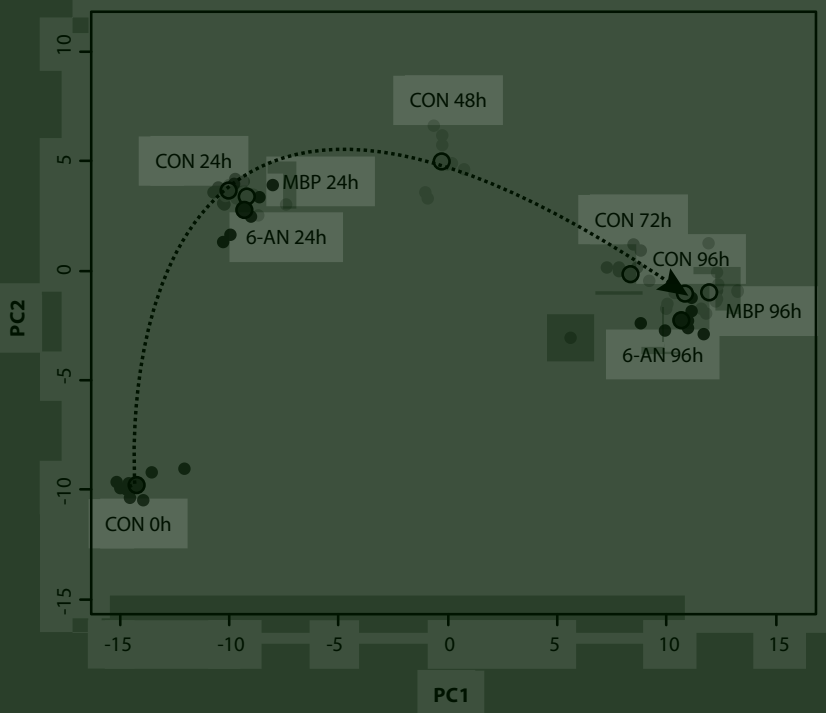

Figure 4. Principal Component Analysis (PCA) of the control cultures after $0,24,48,72$ and $96 \mathrm{~h}$ of differentiation, and cultures exposed to monobutyl phthalate (MBP) or 6-aminonicotinamide (6-AN) for 24 or $96 \mathrm{~h}$. The PCA is based on the 1355 regulated genes. The mean values per experimental group are indicated by the large circles and the 'differentiation track' is shown by the dashed line. For color figure, see Color figures section.

A
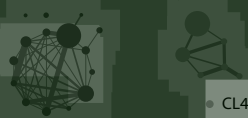

- CL4_24H_UP

Cardiac gene set; downregulated

Pluripotency gene set; upregulated
B

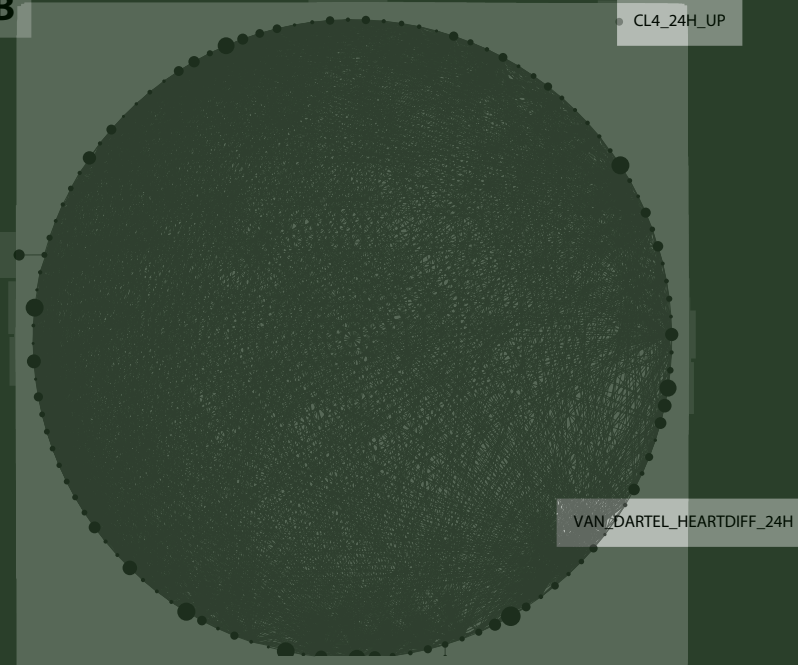

Figure 5. Molecular concept analysis of regulated gene sets (A) after $24 \mathrm{~h}$ monobutyl phthalate exposure in differentiation cultures and (B) after $24 \mathrm{~h} 6$-aminonicotinamide exposure in differentiation cultures. Per set the top $20 \%$ highest affected genes were used. The size of the gene set corresponds with the size of the node, and the width of the connecting lines indicates the degree of overlap between the respective gene sets. For color figure, see Color figures section. 
Compound-induced gene expression changes in differentiating ESC

Comparison of gene expression profiles of differentiation cultures exposed to MBP or 6-AN with their time-matched controls revealed no significant changes in the expression of individual genes. However, GSEA showed that exposure of differentiation cultures to MBP or 6-AN resulted in significant effects at the level of gene set regulation. MBP exposure induced the up-regulation of 14 gene sets that comprise genes involved in pluripotency and differentiation (Figure 5A and Supplemental Table 2). Further analysis using Anni 2.0 revealed that genes that contributed to the significance of this gene set were enriched for processes involved in adhesion, stress, and apoptosis. The seven down-regulated gene sets were mainly enriched for genes involved in early differentiation. Cluster 4 ('Cl4_24h_up', Figure 2), including genes up-regulated after 24h in unexposed differentiation cultures, was significantly down-regulated by 24-h MBP treatment ( $p$-value $<0.001$ and $\mathrm{FDR}<0.001$ ).

6-AN exposure of differentiation cultures resulted in up-regulation of 115 gene sets after $24 \mathrm{~h}$ in comparison to unexposed cultures (Supplemental Table 2). Molecular concept analysis revealed that these sets have a significant overlap in gene content as shown in Figure 5B. These gene sets were predominantly involved in either pluripotency or early differentiation. The only gene set down-regulated in cultures exposed to 6-AN for $24 \mathrm{~h}$ was cluster 4 ('Cl4_24h_up', Figure 2; $p$-value $<0.001$ and FDR $<0.001$ ).

GSEA results of the 96-h exposed cultures were not used for the interpretation of exposure effects because the 'differentiation track' is less dynamic at that time point than at the $24 \mathrm{~h}$ point, as illustrated by the relatively small changes in expression pattern between 72 and $96 \mathrm{~h}$ (Figures 2 and 4).

Compound exposed differentiation cultures deviate from differentiation track Inhibition of cardiomyocyte differentiation was further studied by analyzing the compound-induced deviation from the 'differentiation track' defined by PCA. Both compounds showed statistically significant deviations from the differentiation track. The differentiation cultures exposed for $24 \mathrm{~h}$ to MBP deviated statistically significantly from the first principal component of the 'differentiation track' ( $p$-value<0.04), however after $96 \mathrm{~h}$ of exposure the deviation was not significant anymore ( $p$-value $<0.06)$. Alternatively, the 6-AN exposed differentiation cultures at both time points resulted in a statistically significant deviation from the second principal component of the 'differentiation track' (24 h: $p$-value <0.03, $96 \mathrm{~h}$ : $p$-value $<0.02$; Figure 4 and Table 1). These results show that using the 'differentiation track' of ESC differentiation cultures in the PCA-plot can be used to identify differentiation-modulating effects of two diverse developmental toxicants. 


\section{Discussion}

ESC differentiation and gene expression changes

Gene expression analysis during a 96-h period of unexposed ESC differentiation revealed 1355 statistically significantly regulated genes, which could be divided into seven clusters dependent on their gene expression profile in time. We identified the predominant function of these gene clusters in relation to ESC differentiation as described earlier $[27,109,131,167]$. Analysis of gene function generally indicated loss of pluripotency and gain of cardiac differentiation. This supports the notion that in our gene expression analysis we are clearly monitoring cardiac differentiation.

To our knowledge, this is the most comprehensive analysis to date of gene expression in the early phase of cardiomyocyte differentiation, represented by the 24 and $48 \mathrm{~h}$ time points. This period of the differentiation trajectory appeared to be highly dynamic, as both at 24- and at 48-h (clusters 4 and 5 respectively in Figure 2 ) transiently up-regulated genes were identified. Although genes known to be involved in development were present in cluster 4, such as Hoxb6, Hand1, and BMP4, various groups of genes with the same abundance but with alternative functional characteristics were identified on the basis of the current literature. This indicates that cluster 4 includes genes involved in several biological processes, apparently related to development, which have not been previously described in literature. For cluster 5 no predominant function could be identified, indicating that we have encountered a cluster with a novel functionality for the genes involved.

In a previous study of early cardiac differentiation in ESC, we identified a gene set, 'Van_Dartel_heartdiff_24h', containing 43 genes up-regulated after $24 \mathrm{~h}$ from suspension culture onwards as compared with the 6- and 12-h differentiation time points [111]. In the present study we found that 22 of these 43 genes were already statistically significantly down-regulated at the individual gene level at the 24-h compared to the 0 -h time point. This shows that the differentiation process in the present study was further advanced as compared to our previous study [111]. The discrepancy between both studies can be explained by the rapid, brief and transient up-regulation of the 'Van_Dartel_heartdiff_24h' gene set, similar to the dynamics of cluster 4 ('Cl4_24h_up') at $24 \mathrm{~h}$ of differentiation in the present study. No less than $79 \%$ of the genes in the 'Van_Dartel_heartdiff_24h' gene set were also found statistically significantly regulated in the current study. Further studies will enable the selection of the most reliable genes in terms of reproducible expression changes as a robust set of differentiation markers. Together, these findings show that monitoring differentiation in this model that is characterized by rapidly changing gene expression should not rely on just a single gene cluster defined by time-dependent regulation only. Rather, improved description of differentiation process should be based on a combination of gene clusters with different 
expression dynamics in time, giving a more informative picture of the differentiation stage of the cell population in the assay.

The gene set identified in this study contains 1355 genes that were expressed at different stages of cardiomyocyte differentiation. From literature we identified 83 gene sets in which regulation of genes related to cardiomyocyte differentiation were described [26-28, 111, 132-135, 142-145]. The overlap in gene content with our 1355 genes was relatively low, only 3 gene sets contained $>50 \%$ overlap with our gene set. These sets include the set 'Van_Dartel_heartdiff_24h' [111] and two sets from a study of Masino et al. [142] with respectively, $79 \%, 55 \%$ and $50 \%$ of the genes also present in our current set. This relatively high overlap in gene content, appears to be caused by overlap with a specific cluster within our gene set (cluster 1, 7, and 6 respectively). The majority of all gene sets contained genes that correspond with the gene clusters up-regulated after 72 and $96 \mathrm{~h}$ of differentiation (culture days 6 and 7) in this study. Besides the gene sets identified in this study, no other gene set was found that contained genes regulated after 0,24 or 48 h of differentiation (culture days 3, 4, or 5). Combination of gene sets derived from the same article still does not result in a gene set that describes these stages of differentiation. Therefore, our gene set is currently the most complete one available to study the ESC differentiation dynamics.

We studied the ESC differentiation dynamics by performing PCA on the dataset using the 1355 regulated genes, which showed a continuous PCA trajectory that defines the differentiation track. Using these genes not only the classical end point of cardiomyocyte differentiation is included, but also other biological processes which proceed and/or accompany cardiomyocyte differentiation are incorporated in this differentiation track. Interference with these additional identified biological processes may finally lead to disturbed cardiomyocyte differentiation, and therefore inclusion of genes involved in these other processes may improve the developmental toxicity prediction. This 'differentiation track' was subsequently used as a differentiation fingerprint for the discrimination of developmental toxicant-exposed cultures. This method was also used in other studies to discriminate experimental groups. Van de Waterbeemd et al. [168] used this PCA-method to study the optimal harvest point in bacterial vaccine production, and Liu et al. [169] described the detection of endogenous signalling activation pathways by an oncogenic stimulus.

Compound-induced gene expression changes in differentiating ESC

Studying regulation of individual genes induced by developmental toxicant exposure has shown to be insufficient in developmental models. Developmental modulation restricted to specific cell types may not necessarily lead to statistically significant single gene expression changes because of the dilution of effects occurring in a single cell type within the whole sample that contains a variety of cell types. Kultima et al. [146] 
found only nine genes regulated in day 8 p.c. embryos after exposure to cadmium. In our previous study on differentiation modulation, no individual genes were regulated after MBP exposure [111], and in the present study again no individual genes were regulated after MBP or 6-AN exposure. Therefore, we studied the modulation of the cardiomyocyte differentiation process by analyzing the deviation of MBP or 6-AN exposed cultures from the 'differentiation track' defined by PCA. The PCA approach combines all gene expression data and therefore greatly increases the statistical power of the analysis. When the exposed differentiation cultures were projected onto the two-dimensional expression space delineated by the 1355 differentiation-related genes, it appeared that both MBP and 6-AN deviated significantly from the 'differentiation track' after $24 \mathrm{~h}$ of exposure, confirming the modulating effects of these developmental toxicants on the cardiomyocyte differentiation process. In the early phase of cardiomyocyte differentiation, the differentiation-induced gene expression regulation appeared to be more dynamic as compared to the later phase, which results in a better detection of developmental toxicants after the relatively short exposure duration of $24 \mathrm{~h}$, compared to $96 \mathrm{~h}$.

The GSEA analysis reveals effects on differentiation after $24 \mathrm{~h}$ of compound exposure, using the time-matched cluster 4 ('Cl4_24h_up') defined in the present study. This gene set was strongly down-regulated by both MBP and 6-AN. The gene set 'Van_Dartel_heartdiff_24h', down-regulated after 24h of differentiation in our previous study, was not down-regulated in cultures exposed for $24 \mathrm{~h}$ to MBP and was even up-regulated after $24 \mathrm{~h}$ of exposure to 6-AN (Figure $5 \mathrm{~A}$ and $5 \mathrm{~B}$ ). This can be explained by the fact that differentiation stage of the cultures between experiments can vary, as discussed above [111]. Since GSEA relies on a comparison of only two groups, only time-matched exposed and control groups can be compared to study exposure effects. This cross-section approach is therefore less suitable to identify effects on a dynamic differentiation model. In addition, the genes responsible for significant regulation of the gene set do not per definition have to be involved in the biological process for which the gene set was identified. This was observed in some of the up-regulated gene sets after $24 \mathrm{~h}$ of exposure to MBP compared to control. These gene sets were derived from studies in which gene sets involved in cardiac differentiation were described [133, 145]. Further analysis of the genes within the set responsible for the significant regulation revealed that genes involved in processes as adhesion, stress, and apoptosis contributed to the significance of gene set up-regulation in the MBP exposed cultures. However, in this analysis cardiomyocyte-specific genes within the set, such as Pln, Myl3, and Creld1, did not contribute to the significance of the gene set regulation. Taken together, GSEA does support the rationale that differentiation-related gene sets are affected by compound exposure.

The differentiation track, defined by PCA, described in this study is a more powerful starting point to detect differentiation-related gene expression changes. A significant 
improvement of this 'differentiation track' approach is that genes are included that are regulated at different stages during the entire $96 \mathrm{~h}$ of monitored differentiation. This removes the necessity of using time-matched gene sets for separate experiments. In addition, this makes the model more flexible, as variation in differentiation progression between experiments will not hamper the interpretation of results. Furthermore, when deviation from the 'differentiation track' is studied, effects on all biological processes represented in the assay are included in the analysis. This is a wider assessment than that of the 'classical' EST, which is focusses exclusively on inhibition of cardiomyocyte differentiation, which is only a single end point of the developmental cascade. Thus, the 'differentiation track' approach may lead to an improved applicability domain and increased sensitivity of the EST.

In conclusion, the PCA-derived 'differentiation track' approach appeared to be a suitable method to describe the dynamics of the cardiomyocyte differentiation. Furthermore, this approach provides an important proof-of-principle by showing that the effects of both MBP and 6-AN in the EST could be described by a statistically significant deviation from the 'differentiation track' defined by gene expression changes. It is known from in vivo studies that both MBP and 6-AN cause effects on tissues of mesodermal origin, probably via a different mechanism. The deviation of the compound-exposed cultures from the 'differentiation track' is therefore in line with the in vivo embryotoxicity. Additional testing of non-embryotoxic and embryotoxic compounds that affect tissues of nonmesodermal origin might lead to refinement of the gene set for the prediction of embryotoxicity. Further refinement might be achieved by focussing on the early and highly dynamic phase of the differentiation track.

\section{Acknowledgements}

This study was supported by grant MFA6809 from the Dutch technology society foundation STW.

\section{Supplementary data}

Supplementary data associated with this article can be found at doi:10.1016/j. taap.2009.12.021. 
Dorien A.M. van Dartel, Jeroen L.A. Pennings, Liset J.J. de la Fonteyne, Marcel H. van Herwijnen, Joost H. van Delft, Frederik J. van Schooten, Aldert H. Piersma

Toxicological Sciences (2010) 116:130-9 
CHAPTER 5

Monitoring developmental toxicity in the Embryonic Stem cell Test using differential gene expression of differentiation-related genes 


\begin{abstract}
The Embryonic Stem cell Test (EST) has been designed to predict developmental toxicity based upon compound-induced inhibition of embryonic stem cell (ESC) differentiation. The end point scoring, the test duration, and the definition of the predictivity and the applicability domain require improvements to facilitate implementation of the EST into regulatory testing strategies. The use of transcriptomics to study compoundinduced differentiation modulation may improve the EST in each of these aspects. ESC differentiation was induced and cell samples were collected after 0,24 and $48 \mathrm{~h}$ of differentiation. Additionally, samples were collected that were $24 \mathrm{~h}$-exposed to one of five developmentally-toxic compounds or a non-developmentally-toxic compound. All samples were hybridized to Affymetrix GeneChips, and analyses revealed that 26 genes were significantly regulated both during ESC differentiation and by exposure to each of the developmentally-toxic compounds tested. Using Principal Component Analysis we defined a 'differentiation track' on the basis of this gene list, which represents ESC differentiation. We showed that significant deviation from the 'differentiation track' was in line with the developmental-toxic properties of the compounds. The significance of deviation was analyzed using the Leave-One-Out cross validation, which showed a favorable prediction of toxicity in the system. Our findings show that gene-expression signatures can be used to identify developmental-toxicant induced differentiation modulation. In addition, studying compound-induced effects at an early stage of differentiation combined with transcriptomics leads to increased objectivity in determining differentiation inhibition and to a reduction of the test duration. Furthermore, this approach may improve the predictivity and applicability domain of the EST.
\end{abstract}




\section{Introduction}

The implementation of the new EU regulation for Registration, Evaluation and Authorization of Chemicals (REACH) in 2007 will bring about the assessment of the potential toxicity of thousands of chemicals within the coming years. The current regulatory guidelines for the toxicological hazard identification require large numbers of experimental animals. As the highest number of experimental animals are currently being used in regulatory reproductive and developmental toxicology [119], the demand for in vitro alternative test methods is particularly high in that field. Various alternative testing methods for the prediction of developmental toxicity have already been developed. These tests vary from whole embryo cultures of rat or zebrafish, and organ cultures, to continuous cell-line-based tests, such as the Embryonic Stem cell Test (EST) [19, 84-86].

To effectively reduce the demand for experimental animals, in vitro alternative test methods need regulatory acceptance. The validation by the European Centre for Validation of Alternative Methods (ECVAM) of the EST, the rat Whole Embryo Culture and the MicroMass as alternative testing methods has been an important exercise towards this regulatory acceptance so far. Of these three tests, the EST is the only one that is completely animal-free. However, while the EST is considered a promising alternative assay for developmental toxicity, it has several weaknesses as recently discussed in an ECVAM Workshop [170]. Aspects that need optimization to allow successful regulatory implementation include culture duration, end point scoring, and the definition of its predictivity and its applicability domain.

The EST predicts developmental toxicity based on the inhibition of the differentiation of embryonic stem cells (ESC) into cardiomyocytes. However, besides these mesodermal-derived cardiomyocytes, cells derived from both the ecto- and endodermal germ layers also develop within these differentiation cultures $[6,87]$. This resembles the in vivo situation in which cells from each of these three germ layers are present in the developing embryo [171]. The prediction of developmental toxicity by the EST could possibly be optimized if compound-induced interference with each of the cell types present in the EB would be taken into account.

The use of molecular techniques in toxicology has enabled the early detection of changes in gene and protein expression $[172,173]$. In addition, these methods can help in our understanding of the mechanisms of action of toxicity [174, 175]. Small-scale molecular techniques, such as real-time polymerase chain reaction (RT-PCR), can be used to study modulations of the expression of specific genes by a toxicant expected on the basis of knowledge of the mode of action. More advanced techniques, including transcriptomics, can be used to study gene expression alterations in the whole genome, not necessarily requiring prior mechanistic knowledge. The transcriptomic technique has shown promising results in toxicology. Waring et al. [176] revealed a correlation 
between gene expression profiles of hepatoxins and mechanisms of toxicity. In the field of developmental toxicology, Robinson et al. [54] and Jergil et al. [52] showed that compound-induced gene expression changes in in vivo and in vitro systems, respectively, were related to teratogenic effects observed in vivo.

The implementation of transcriptomics in the EST may optimize this test as a successful in vitro model for predicting developmental toxicity. Analyses of gene-expression changes have shown to be feasible within an abbreviated experimental protocol [111, 177]. Furthermore, ESC differentiation-related genes have been identified in these studies, which could help us to define the applicability domain and improve the predictivity of the EST.

In this study, we investigated the possibility of predicting developmental toxicity by the implementation of transcriptomics into the EST. We applied Principal Component Analysis (PCA) of gene expression dynamics to define the 'differentiation track' in differentiating ESC in the EST protocol as previously described [177]. In the present study, we show that five developmental toxicants caused significant deviation from the differentiation track, whereas a non-developmental-toxic negative-control compound did not deviate from the differentiation track. We identified a set of 26 differentiationregulated genes of which the expression was changed both during ESC differentiation and after exposure to each of the five developmental toxicants but not necessarily after exposure to the non-developmentally-toxic compound. Moreover, application of the Leave-One-Out method showed a favorable prediction of toxicity in the system. These findings show that gene expression signatures can be used to describe and possibly predict developmental toxic responses in the EST.

\section{Materials and methods}

Test compounds

Test compounds used in this study were monobutyl phthalate (MBP; CAS No. 13170-4, TCI Europe, Zwijndrecht, Belgium), methoxyacetic acid (MAA; CAS No. 625-45-6, Sigma-Aldrich, Zwijndrecht, The Netherlands), valproic acid (VPA; CAS No. 1069-66-5, Sigma-Aldrich), and retinoic acid (RA; CAS No. 302-79-4, Fluka, Buchs, Switzerland), which were dissolved in dimethyl sulfoxide (DMSO), and 5-fluorouracil (5-FU; CAS No. 51-21-8, Sigma-Aldrich), and Penicillin G (PenG; CAS No. 69-57-8, Sigma-Aldrich), which were dissolved in phosphate-buffered saline (PBS).

Resazurin cell proliferation assay

Compound-induced effects on cell number were determined using resazurin dye reduction, which is a measure for the number of viable cells per well [178], as described previ- 
ously [150]. In short, hanging drop cultures were set up using pluripotent murine D3 embryonic stem cells (ESC; ATCC, Rockville, MD) and exposed to a concentration range of the selected test compounds. After 3 days, embryoid bodies (EB) were harvested per compound concentration and resazurin (Promega, Madison, $\mathrm{WI}$ ) was added to the cells. The resazurin reduction by the cells was measured after incubation at $37^{\circ} \mathrm{C}$ at $530 \mathrm{~nm}$ (excitation) and $590 \mathrm{~nm}$ (emission) using a FLUOstar Galaxy microplate reader (BMG Lab Technologies, Offenburg, Germany). The resazurin reduction of exposed cells relative to control measurement was calculated and plotted against the test concentrations using PROAST software [103]. For each compound a minimum of five concentrations were tested in three independent runs. The cell survival at the concentration used in the transcriptomic experiment was calculated for each compound on basis of the curve fit.

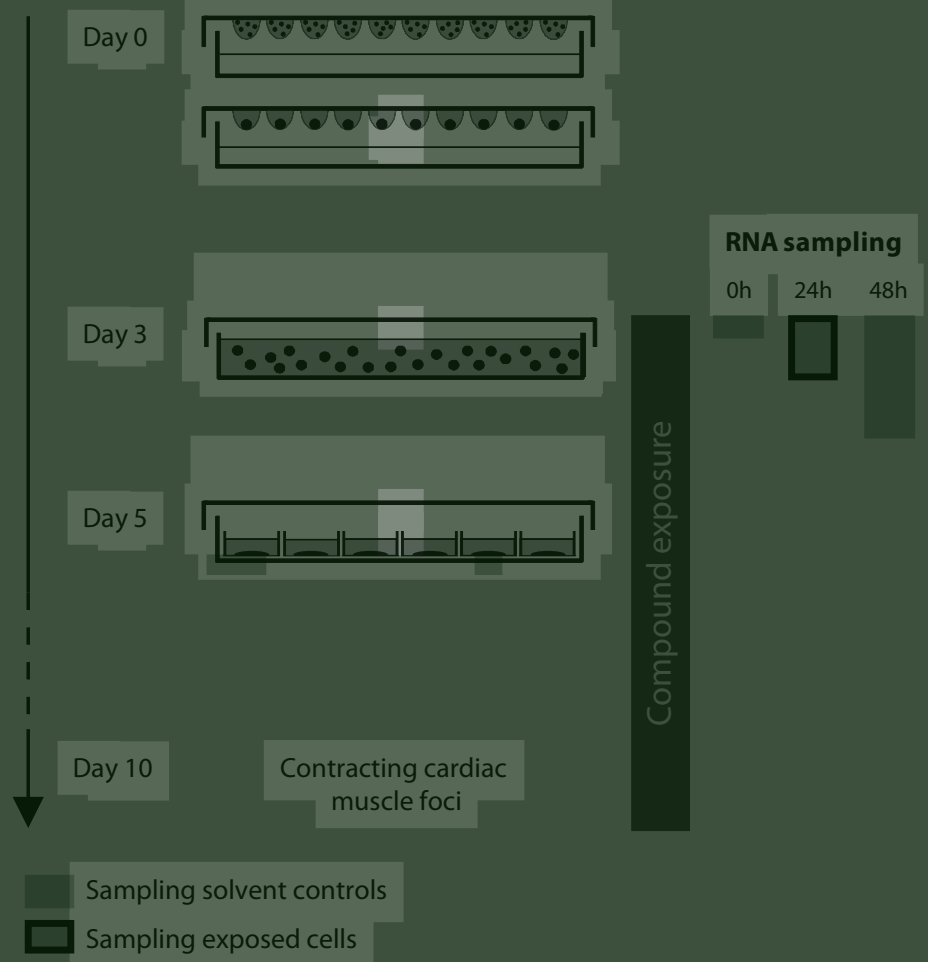

Figure 1. Schematic overview of experimental procedures of cardiomyocyte differentiation. Hanging drop cultures containing a suspension of single embryonic stem cells were set up at day 0 . The dissociated cells formed embryoid bodies (EB), which were transferred to suspension culture plates at day 3. At day 5, EB were plated one per well into 24-well tissue culture plates. At day 10, each EB was microscopically evaluated for the presence of contracting cardiomyocyte foci. Differentiating cells were exposed to test compounds from day 3 onwards. For transcriptomic analysis, non-exposed control samples were isolated after 0,24 , and $48 \mathrm{~h}$ of exposure, and compound-exposed cultures were sampled after $24 \mathrm{~h}$ of exposure. 
Pluripotent embryonic stem cell culture and cardiomyocyte differentiation

D3 ESC were cultured in a monolayer in complete medium supplemented with leukemia inhibitory factor (LIF; Chemicon, Temecula, CA), as described previously [7, 177]. Embryoid bodies (EB) were obtained via hanging drop culture in complete medium without LIF $[7,102,177]$ as is visualized in Figure 1 . These EB cultures were used as the primary step for ESC differentiation. The cardiomyocyte differentiation was considered successful if in at least 21 out of the 24 evaluated control EB, at least one contracting focus was present.

Inhibition of cardiomyocyte differentiation by compound exposure

ESC differentiation cultures were exposed from the EB-stage at day three onwards to concentrations that were calculated (on the basis of historic data) to inhibit cardiomyocyte differentiation with $50 \%$ compared to control cultures. All cultures were exposed to $0.2 \%$ DMSO for comparability reasons. Cultures were microscopically evaluated at day 10 of the culture to assess the inhibition of cardiomyocyte differentiation (Figure 1), while parallel cultures were used for gene expression analysis at earlier time points as explained below.

RNA isolation and whole genome gene expression profiling

RNA of compound-exposed cultures was isolated $24 \mathrm{~h}$ after the start of exposure, which corresponds with culture day 4 (6-8 replicates per group). dimethyl sulfoxide (DMSO)exposed control differentiation cultures were harvested after 0,24 and $48 \mathrm{~h}$ from the EBstage at day 3, which corresponds with culture day 3, 4, and 5 (12 replicates per group) as is visualized in Figure 1. Cells were directly collected in RNA protect (Qiagen, Venlo, The Netherlands) to stabilize RNA, and total RNA was purified using the RNeasy mini kit (Qiagen, Venlo, The Netherlands) with an additional DNase treatment (RNase-Free DNase Set; Qiagen, Venlo, The Netherlands) according to the manufacturer's instructions. The quantity of RNA in each sample was measured using a NanoDrop Spectrophotometer (NanoDrop technologies, Wilmington, DE) and RNA integrity was assessed on the 2100 Bioanalyzer (Agilent Technologies, Amstelveen, The Netherlands) using the RNA 6000 Nano Chip Kit (Agilent Technologies) and good quality RNA was used for gene expression analysis (RNA Integrity Number (RIN) >8). Of the corresponding RNA samples, 250 ng was amplified and fragmentated using the Affymetrix GeneChip ${ }^{\circledR}$ 3'IVT Express Kit (Affymetrix, Santa Clara, CA) according to manufacturer's instructions on the Xiril Neon 150 robotic system (GC-Biotech, Alphen a/d Rijn, The Netherlands). Next, $250 \mu \mathrm{l}$ hybridization cocktail was added to $12.5 \mu \mathrm{g}$ fragmented aRNA and $200 \mu \mathrm{l}$ of this cocktail was applied to the Mouse Genome 4302.0 arrays (Affymetrix) and hybridized for $16 \mathrm{~h}$ at $45^{\circ} \mathrm{C}$ in a Genechip Hybridization Oven 640 (Affymetrix). After hybridization the arrays were washed and stained with a Genechip Fluidics Station 450 (Affymetrix) using the Genechip hybridization wash and stain kit. Thereafter, the arrays were scanned using 
the Genechip scanner 3000 (Affymetrix). Quality controls were performed according to the manufacturer's instructions and showed to be within acceptable limits for all arrays, except for one RA-exposed sample that was excluded for further analysis.

Data analysis and statistics

Affymetrix CEL files were normalized using the Robust Multichip Average (RMA) algorithm [179] using RMAexpress [180]. For probe to gene mapping, a custom Chip Description File (CDF) was used according to the recent assembly by de Leeuw et al. (http://mad-db.science.uva.nl/ wdeleeuw/HybridAnnot/version6.html) [181]. Of the hybrid probe-set definitions included in the custom annotation, 16,331 probe sets defined by the Brainarray custom CDF version 11 (http://brainarray.mbni.med.umich. edu/Brainarray/Database/CustomCDF) [182] and 4,648 additional probe sets defined by Affymetrix chip annotation 26 were used in further analyses, giving a total of 20,979 probe sets. Probe sets for Affymetrix internal controls or probe sets that did not correspond to an Entrez Gene ID were not used in further analyses. Raw and normalized data are deposited at ArrayExpress (http://www.ebi.ac.uk/arrayexpress) under accession number E-TABM-903.

Statistical analyses were carried out using the R statistical software environment [151] using log-transformed values. For each gene, maximal fold ratios (FR) in gene expression between the experimental groups were determined by comparing the average normalized signal values per group and were calculated as the maximum $/$ minimum ratio. Genes that were differentially expressed between any of the experimental groups were identified by a one-way ANOVA on the normalized data. A p-significance threshold of 0.001 and $F R>1.5$ was used to select genes that were significantly differentially expressed when ESC differentiate. A $p<0.001$ without FR restriction was applied for the identification of significantly differentially expressed genes caused by compound exposure.

Arrangement of the genes that were significantly differentially expressed in the ESC differentiation cultures in expression clusters was achieved by hierarchical clustering based on Euclidian distance and Ward linkage, using GeneMaths XT (Applied Maths, Sint-Martens-Latem, Belgium). Functional annotation and enrichment for Gene Ontology (GO) biological processes were studied using the tools on the DAVID website [183]. Only genes with FR>1.5 were used to study this enrichment due to DAVID's limitation on the length of the gene-list input. In order to perform functional annotation of individual genes, we used the Entrez gene database on the NCBI website (http://www.ncbi.nlm. nih.gov/gene/).

Definition of the differentiation track

Using the genes significantly differentially expressed in ESC differentiation, we defined the 'differentiation track' of normal ESC differentiation as we described previously [177]. 
This approach is based on Principal Component Analysis (PCA) [58], which is a mathematical algorithm that describes the data on the basis of their (dis)similarity, so that a greater distance corresponds with a greater dissimilarity. In this analysis, a principal component is defined as a mathematically derived combination of genes and their expression characteristics that can be used to describe part of the process observed. A number of principal components that are mutually independent can be derived which in combination describe the process under study. At first a gene list was defined, which comprised the genes that were identified to be significantly differentially expressed among the ESC differentiation groups sampled at different stages of ESC differentiation. Next, PCA analysis was performed using this gene list, using R. We connected a line between the average array gene expression at 0,24 and 48h on our PCA plot to display the continuous representation of the ESC differentiation based on the dynamics of gene expression. This curve was defined as the 'differentiation track'. Later, the number of genes on this list was reduced by selecting the genes that were both regulated in ESC differentiation and by compound exposure. Finally, this refined gene list was used to describe the ESC differentiation by means of the PCA-derived differentiation track. Coordinates along the first and second principal component were calculated for each sample. Deviation of compound exposed cultures from the 'differentiation track' was analyzed by applying a Hotelling T-test to these coordinates compared to those of timematched control cultures. Significant deviation from the 'differentiation track' $(p<0.05)$ was considered characteristic for developmental toxicants.

To cross-validate the classification by the obtained gene list using the 'differentiation track' approach, we used the Leave-One-Out validation test. Expression data of compound-exposed experimental groups were left out one-by-one to obtain the genes that were regulated in ESC differentiation as well as after exposure to all other developmental toxicants. These gene lists were used to define the 'differentiation track' and for each compound-exposed experimental group the statistical significance of deviation from these differentiation tracks was calculated.

\section{Results}

Compound-induced effects on cell proliferation and differentiation

Differentiating ESC were exposed to single test compound concentrations, selected on the basis of historic data from our laboratory. Effects on proliferation and differentiation were scored in cultures parallel to those analyzed for gene expression. The effects on proliferation at day three of hanging drop culture and on cardiac foci scoring at termination (day 10) of EST culture are given in Figure 2. PenG, VPA and MBP did not affect cell survival, whereas RA, MAA and 5-FU reduced cell numbers in varying degrees. All 


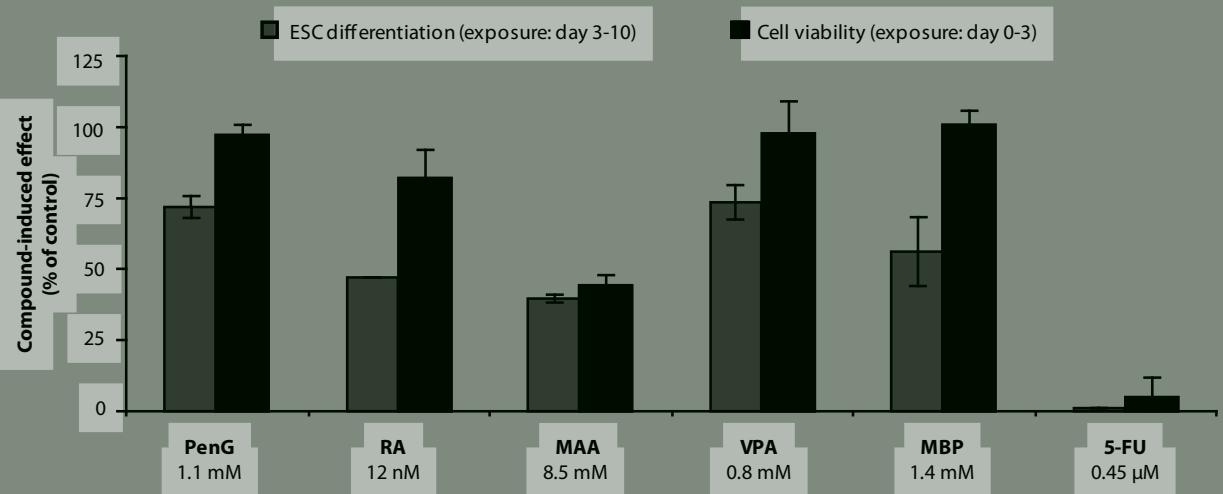

Figure 2. Effect of compound exposure on cell survival $(n=3)$ and beating cardiomyocyte differentiation $(n=2)$ at the concentrations tested in the transcriptomic experiment (mean $\pm s d)$.

compounds affected differentiation, with PenG and VPA as the least effective and 5-FU as the most effective compound tested. The remaining three compounds showed differentiation inhibition of around $50 \%$.

Differentiation-related gene expression changes

The gene expression profiles of the samples of unexposed differentiation cultures at 0,24 , and $48 \mathrm{~h}$ were compared by means of a one-way ANOVA analysis, using cut-off criteria of $p<0.001$ (corresponding False Discovery Rate $=0.0017$ ) and FR $>1.5$. We identified 3579 genes showing statistically significant differential expression with time. This gene expression pattern showed the dynamics of early cardiomyocyte differentiation as shown in Figure 3A. Broadly, three main gene clusters were observed based on the gene expression dynamics of the differentiating ESC, representing the highest differential gene expression values among the time points studied. These gene clusters were enriched for developmental-related terms, including cell differentiation $(0 \mathrm{~h})$, somitogenesis ( $24 \mathrm{~h}$ ), developmental process ( $24 \mathrm{~h}$ and $48 \mathrm{~h}$ ), and tube development ( $48 \mathrm{~h}$ ). The gene cluster up-regulated at $\mathrm{Oh}$ was also enriched for cell proliferation and metabolism. Furthermore, the term extracellular matrix was found to be significantly related to the identified gene cluster at 48h (Figure 3A).

Principal Component Analysis (PCA), using the 3579 transcriptionally responsive genes, showed that $94.0 \%$ of all variance between the unexposed experimental groups could be described using only two principal components of the PCA (Figure 3B). Additional principal components had a minor contribution to total gene expression variance and produced no significant shifts between these experimental groups. The PCA plot clearly shows the differentiation dynamics, and indicates that the variance within experimental groups is relatively very small as compared to the variance between time points in ESC differentiation (Figure $3 \mathrm{~B}$ ). In addition, the experimental time points appeared 


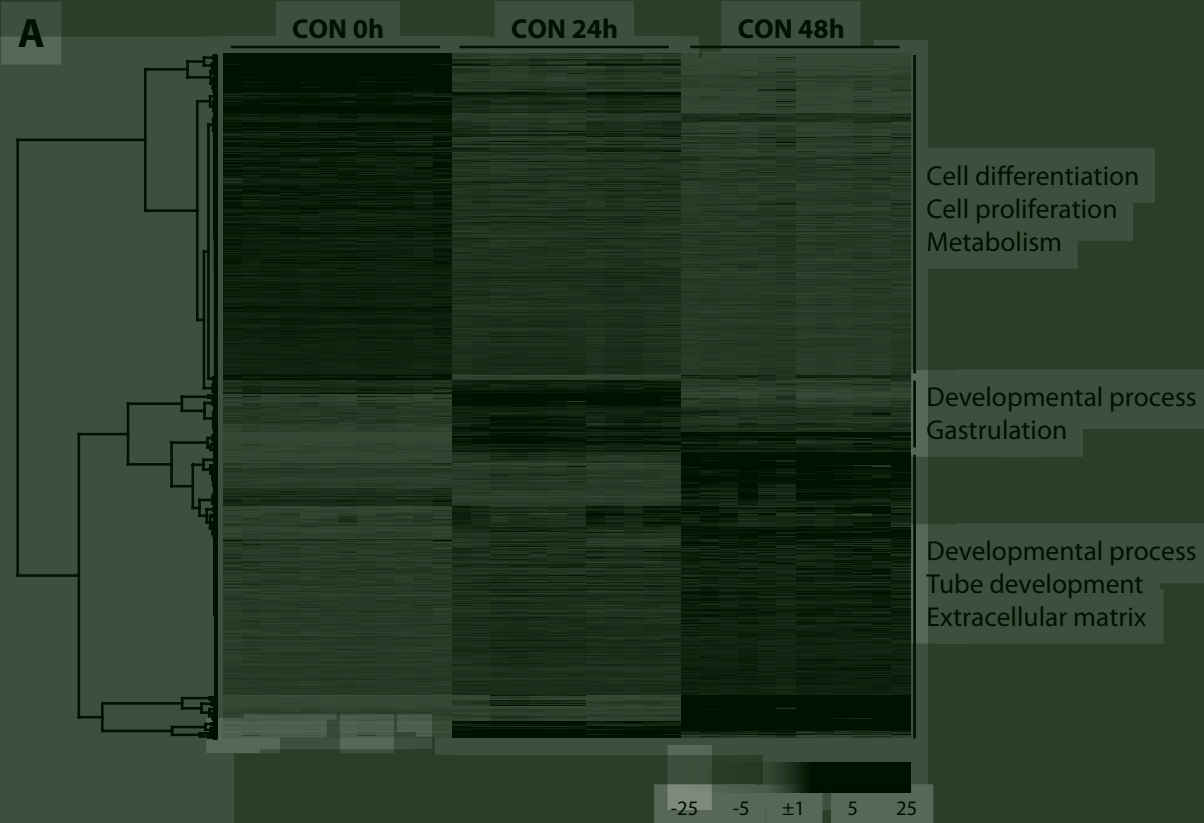

B

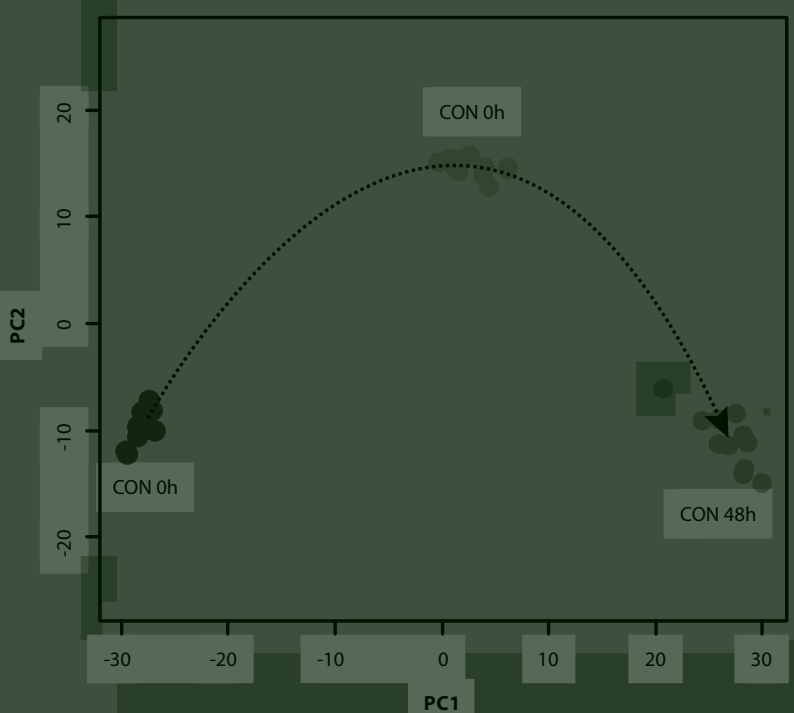

Figure 3. (A) Clustering of the 3579 genes transcriptionally responsive to ESC differentiation between 0 , 24 and $48 \mathrm{~h}$. Each row represents the relative expression of a single gene throughout differentiation. (B) Principal Component Analysis of unexposed control cultures (CON) after 0, 24 and 48h of differentiation using the 3579 differentiation-related genes. The arrow connecting these experimental groups represents the differentiation track. For color figure, see Color figures section. 


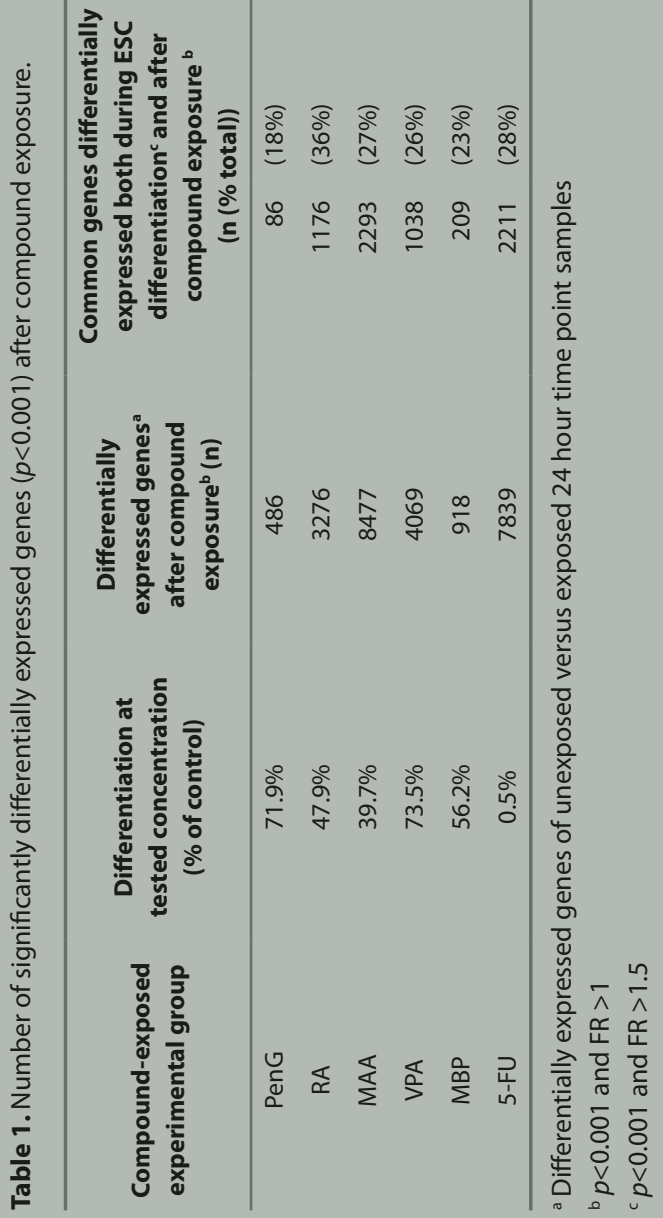




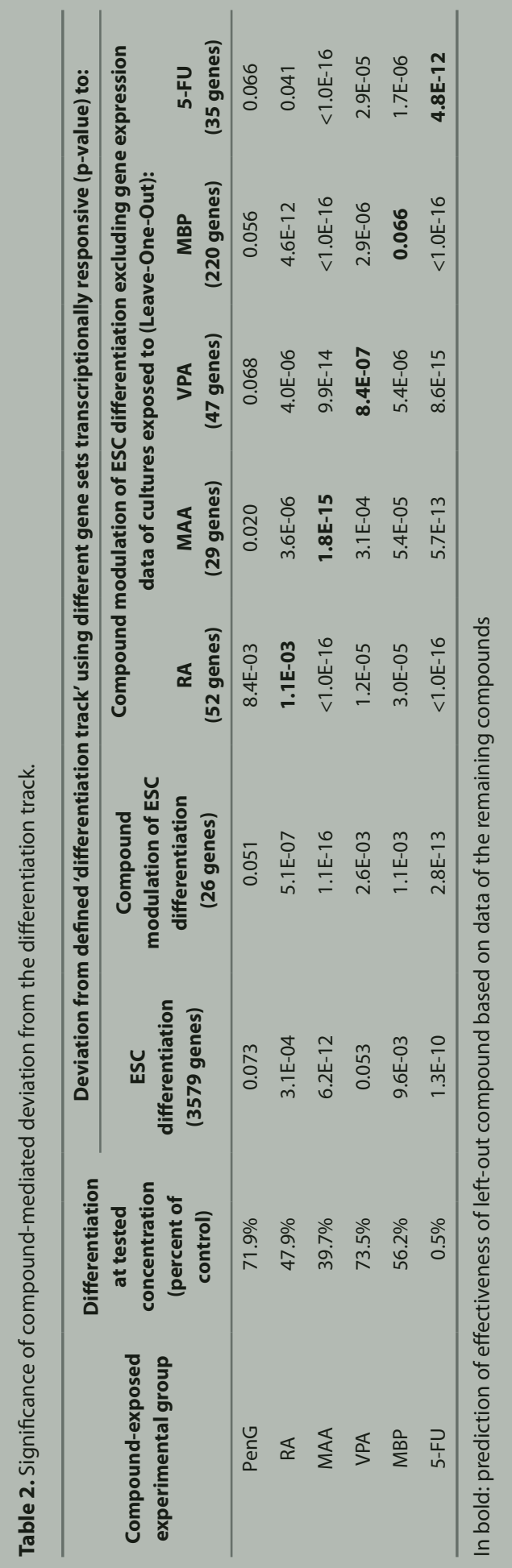


in chronological order in the PCA plot and a curve connecting these points showed a continuous PCA trajectory which can be considered as the differentiation track.

Compound-induced gene expression changes

Comparison of the gene expression profiles of the compound-exposed experimental groups with the time-matched control group revealed the significantly differentially expressed genes per treatment $(p<0.001$; Table 1$)$. The highest numbers of genes were affected by exposure to MAA (8477 genes) and 5-FU (7839 genes). Furthermore, the expression of a relatively high number of genes was changed after exposure to VPA (4069 genes), RA (3276 genes), and MBP (918 genes), while the non-developmental toxicant PenG exposure altered the regulation of only 486 genes.

$\mathrm{GO}$ enrichment revealed down-regulation of developmental-related processes for all exposures, except for cultures exposed to MBP and to the negative control PenG. Besides this down-regulation, developmental-related processes were also up-regulated in RA-and VPA-exposed cultures, such as pattern specification process (RA), regionalization (RA), and neurite development (VPA), which is clearly indicative for these compoundinduced differentiation disturbances. Among the down-regulated genes in MAA- and 5-FU-exposed cultures we observed a significant enrichment in proliferation-related genes, which is in line with their observed decrease in cell number after exposure.

For all developmental-toxicant exposed groups a significant proportion (23-36\%) of the regulated genes showed overlap with the 3579 genes that were found to be differentially expressed in ESC differentiation (Table 1), whereas this percentage of common genes was limited to $18 \%$ in PenG-exposed cultures. Evaluating the common genes regulated by compound exposure and by differentiation using only the more extensively regulated genes (FR>1.5) supported these observations. At least $50 \%$ of the genes regulated by developmental toxicants shared overlap with the genes regulated in differentiation. In contrast, in the PenG-exposed cultures no single gene was regulated with FR $>1.5$, and subsequently no overlap could be calculated (data not shown). Moreover, significant overlap was found between the significantly differentially expressed genes of any two compounds tested (Table 1).

Compound-induced deviation from differentiation track

Differentiation modulation by compound exposure was further studied using the 'differentiation track' approach. First, the deviation from time-matched non-exposed cultures was calculated for all exposed groups (Table 2). The significance levels of the $p$-values correlated with the level of differentiation inhibition observed via morphological scoring. The cardiomyocyte differentiation of cultures exposed to VPA was only decreased to $73.5 \%$, this was mimicked by the borderline non-significant deviation of this experimental group from the 'differentiation track' $(p=0.053)$. On the other hand, the 
Table 3. Overview of the 26 genes transcriptionally responsive to both ESC differentiation and developmental toxicant exposure.

\begin{tabular}{|c|c|c|c|}
\hline $\begin{array}{l}\text { Entrez } \\
\text { Gene ID }\end{array}$ & Gene Symbol & Gene Name & Function \\
\hline 109857 & Cbr3 & Carbonyl reductase 3 & Xenobiotic metabolism \\
\hline 664968 & $2210411 K 11 R I K$ & RIKEN cDNA $2210411 \mathrm{~K} 11$ gene & Unknown \\
\hline 211896 & Depdc7 & DEP domain containing 7 & Unknown \\
\hline 107515 & Lgr4 & $\begin{array}{l}\text { Leucine-rich repeat-containing G protein- } \\
\text { coupled receptor } 4\end{array}$ & Development \\
\hline 66995 & Zcchc18 & Zinc finger, CCHC domain containing 18 & Unknown \\
\hline 19301 & Pxmp3 & Peroxisomal membrane protein 2 & Unknown \\
\hline 114714 & Rad51c & RAD51 homolog c (S. cerevisiae) & DNA repair \\
\hline 19183 & Psmc3ip & $\begin{array}{l}\text { Proteasome (prosome, macropain) } 26 \mathrm{~S} \\
\text { subunit, ATPase } 3 \text {, interacting protein }\end{array}$ & Unknown \\
\hline 66953 & Cdca 7 & Cell division cycle associated 7 & Cell division \\
\hline 18045 & Nfyb & Nuclear transcription factor-Y beta & Transcription \\
\hline 72672 & Zfp518 & Zinc finger protein 518 & Transcription \\
\hline 22249 & Unc13b & Unc-13 homolog B (C. elegans) & Apoptosis \\
\hline 53868 & Rab25 & RAB25, member RAS oncogene family & Unknown \\
\hline 17349 & Mlf1 & Myeloid leukemia factor 1 & Cell differentiation, proliferation \\
\hline 329727 & Dennd2c & DENN/MADD domain containing $2 \mathrm{C}$ & Unknown \\
\hline 16173 & 1118 & Interleukin18 & Immune response \\
\hline 69635 & Dapk1 & Death associated protein kinase 1 & Development, apoptosis \\
\hline 193796 & $K d m 4 b$ & lysine (K)-specific demethylase 4B & Unknown \\
\hline 56786 & Tmem9b & TMEM9 domain family, member B & Unknown \\
\hline 13823 & Epb4.113 & Erythrocyte protein band 4.1-like 3 & Cytoskeleton \\
\hline 20913 & Stxbp4 & Syntaxin binding protein 4 & Metabolism \\
\hline 242362 & Manea & Mannosidase, endo-alpha & Protein glycosylation \\
\hline 14958 & HifO & $\mathrm{H} 1$ histone family, member 0 & Histone \\
\hline 74134 & Cyp2s1 & $\begin{array}{l}\text { Cytochrome } \mathrm{P} 450 \text {, family } 2 \text {, subfamily s, } \\
\text { polypeptide } 1\end{array}$ & Xenobiotic metabolism \\
\hline 213819 & Casd1 & CAS1 domain containing 1 & Unknown \\
\hline 53860 & Sept9 & Septin9 & Cell division \\
\hline
\end{tabular}

high significance values for 5-FU and MAA also correlated with their strong inhibition of the differentiation in parallel cultures scored for morphology at day 10.

Secondly, we defined a minimal subset of genes regulated in both ESC differentiation and by exposure to each of the developmental toxicants. Using these criteria we defined a subset of 26 genes out of the 3579 that were changed during differentiation (Table 3). Gene functions in the gene set include development, transcription and cellular proliferation. For 10 of the 26 genes no information about gene function was available. 


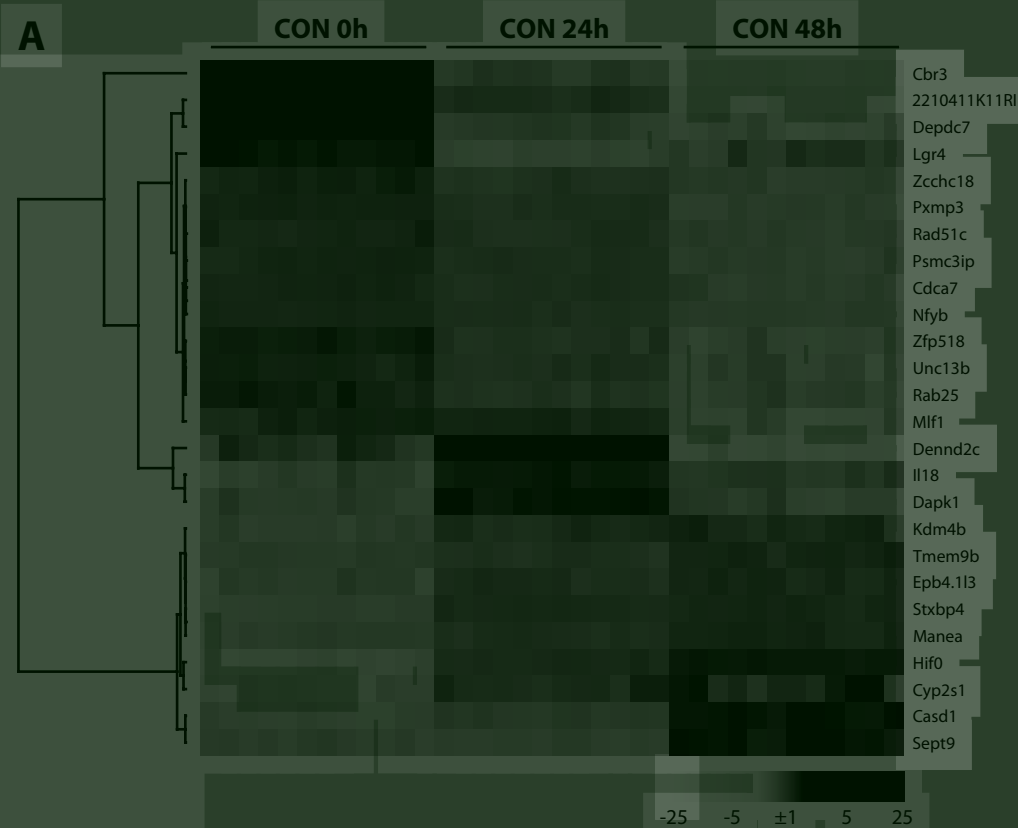

B

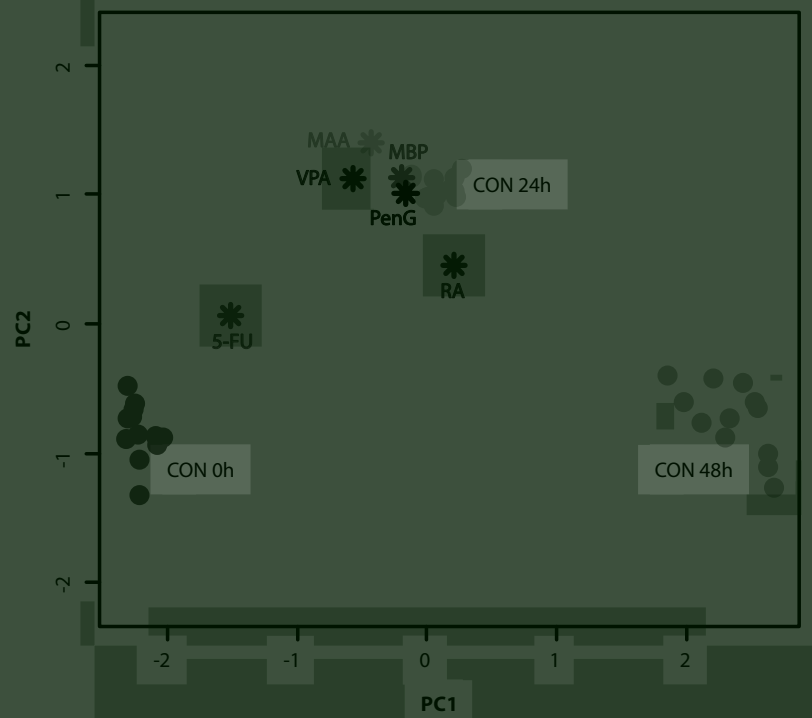

Figure 4. (A) Clustering of the 26 genes that are both transcriptionally responsive to ESC differentiation with time and are also regulated by exposure to all developmental-toxic compounds tested. (B) Principal Component Analysis of the non-exposed cultures (CON) (individual samples, circles) at different stages of differentiation and compound-exposed cultures (mean of $n=8$ samples, asterisks) using the 26 biomarker genes. For color figure, see Color figures section. 
Gene expression dynamics of differentiating ESC could effectively be visualized in a PCA plot using the subset of 26 genes (Figure $4 \mathrm{~A}$ and B). The deviation of the compoundexposed differentiation cultures from the 'differentiation track' based on these 26 genes was calculated and visualized (Table 2, Figure 4B). Using the subset of 26 genes, all developmental toxicants deviated significantly from their time-matched controls on the differentiation track. Furthermore, the negative developmental toxicant, PenG, showed no statistically significant deviation from the $24 \mathrm{~h}$-controls on the differentiation track.

Leave-0ne-Out prediction of developmental toxicity

We analyzed the predictivity of this gene expression dataset for the compounds tested by a Leave-One-Out cross validation (Table 2). Gene subsets were derived composed of the genes that were both regulated in ESC differentiation and by all compounds except the one left out. This resulted in gene subsets of 29 (MAA left out) to 220 (MBP left out) commonly affected genes. Testing the left-out compound on the basis of the gene set generated with the remaining compounds showed that all developmental toxicants studied were scored as significantly deviating from the time-matched controls on the 'differentiation track' (Table 2, numbers in bold) except for MBP ( $p=0.066)$. The significance of differentiation-track deviation of the remaining 25 experimental groups were in line with their expected developmental toxicity except for two of the predictions related to the non-developmental toxicant PenG, with $p$-values of $8.4 \mathrm{E}-03$ and 0.020 , respectively.

\section{Discussion}

In the present study we have applied transcriptomics as a tool to study ESC differentiation and its modulation by a variety of developmental toxicants. We hypothesized that differential gene expression would objectivate effect assessment in the EST. In addition, predictivity might potentially be improved and the applicability domain expanded as gene expression changes include all cell lineages present in the culture. Finally, with this approach culture duration could be abbreviated. Since transcriptomics has been shown to be an attractive approach to study the toxicity of compounds $[52,54,176,177]$, we further studied the implementation of this technique in the EST to improve the model in the above-mentioned aspects.

Genes transcriptionally responsive to ESC differentiation

In the present study we uncovered more than 3500 genes that were regulated in response to ESC differentiation, and this expands the findings of previous microarray studies $[28,111,132,135,142,177]$. The enrichment of this group of genes for 
developmental-related processes, including cell differentiation, gastrulation and tube development, confirms that our model shares important characteristics with in vivo development. These genes may thus provide a good basis for developing a gene set that could be used as a biomarker for developmental toxicity prediction.

Furthermore, despite fundamental differences in experimental design with our previous studies [111, 177], a large fraction ( $84.2 \%$ and $70.5 \%$, respectively) of the genes regulated in early ESC differentiation in those studies were also present in the set of 3579 genes identified in the present study. In addition, a similar pattern of ESC-expression dynamics in time and GO enrichment was observed in the current study as compared to our previous studies, which illustrates the robustness of this differentiation model.

\section{Compound-induced gene expression changes}

All compounds tested in the present study induced alterations in gene expression, however major differences in the number of the regulated genes and the extent of regulation were present. The negative control PenG caused the regulation of 486 genes, however the induction of those genes was modest, i.e. no genes were regulated with a $F R>1.5$. In contrast, the developmental-toxic exposures caused a high number of genes to be regulated and at a higher induction magnitude.

5-FU and MAA, which were demonstrated to affect cell survival and proliferation at the tested concentration, caused large alterations in individual gene expression. Analyses of the significantly regulated genes revealed that the down-regulated genes were enriched for proliferation-related processes. This confirmed that 5-FU and MAA affected cell survival and/or proliferation as is known from literature [97, 98, 184, 185]. In addition, 5-FU and MAA exposure also affected developmental-related processes, which confirms their modulating effects on the ESC differentiation process. Although 5-FU and MAA may indirectly affect differentiation via inhibition of cell proliferation, the detection of such compounds as developmental toxicants is of great relevance.

RA, VPA, and MBP caused the regulation of a relatively high number of genes, which was associated with their differentiation modulating activities as observed via microscopical evaluation of ESC differentiation cultures. GO enrichment analyses using these regulated genes revealed that both up- and down-regulated genes in RA- and VPA-exposed cultures were significantly enriched for developmental-related processes. This clearly illustrates the differentiation-modulating properties of these compounds as described earlier [186-188]. In contrast, MBP-exposed cultures did not reveal disruption of developmental-related processes using these enrichment analyses. We showed previously that incomplete functional annotation of differentiation-related genes may hamper detection of gene function enrichment using standard GO-based tools, including DAVID [111]. Therefore, in that study, we defined a novel defined gene set involved in early differentiation to successfully identify the MBP-induced differentiation-modula- 
tion. This present study also shows regulation of a large portion of ESC differentiation related genes by MBP (23\%). This confirms that customized gene sets have a significant added value in revealing differentiation-modulation using genes that are subtly, but significantly regulated.

Detection of developmental toxicity using the differentiation track

Although developmental toxicants may cover a wide range of possible mechanisms of action, several studies have shown that many developmental toxicants tested shared the ability to modulate cardiomyocyte differentiation in the EST [19, 21, 22]. Therefore, to identify developmental toxicants, we focused on the compound-induced regulation of differentiation-related genes in the EST. In our previous study, we showed that developmental toxicants could be identified using differential gene expression [177]. We employed the PCA-derived 'differentiation track' method to identify developmental toxicants using genes that were transcriptionally responsive to cardiomyocyte differentiation of ESC. In that study, genes were used that were regulated during the first $96 \mathrm{~h}$ of ESC differentiation, however additional analyses showed that the later time points were less informative for the identification of compound-induced differentiation modulation. Using the genes regulated during the first $48 \mathrm{~h}$ of differentiation only improved the significance of deviation from the 'differentiation track' of the developmental toxicants studied. Therefore, in the current study gene expression during the first $48 \mathrm{~h}$ of differentiation only was investigated. Here, the 3579 ESC-differentiation-responsive genes were used to derive the PCA-defined differentiation track. Developmental-toxicant-exposed groups appeared to deviate significantly from the time-matched controls on this track, confirming the modulations of the differentiation process.

Selection of those genes that were both transcriptionally responsive in ESC differentiation and to exposure to each of the developmental toxicants studied resulted in a set of only 26 genes. Importantly, these genes described a similar 'differentiation track' as compared to the 3579 genes during the 48-h period of ESC differentiation studied. Using the refined set of 26 genes, the significance of deviation from the time-matched controls on the differentiation-track appeared to be in line with the developmental-toxic properties of the tested compounds, and for all compounds tested the significance of deviation was increased as compared to the analysis based on all 3579 developmental genes. Further studies are needed to establish whether this descriptive gene set is indeed predictive for other developmental-toxic compounds.

Leave-0ne-0ut cross validation

We applied the commonly used cross-validation according to the Leave-One-Out principle to get a first impression of the predictivity of our gene expression approach. This validation showed that each compound, when its data was not included in refining the 
gene set, deviated significantly from the time-matched controls on the differentiation track, indicating that it was correctly predicted as a developmental toxicant on the basis of the remainder of the data. As the single exception, the deviation of MBP-exposed cultures from its time-matched controls was borderline nonsignificant $(p=0.066)$. This MBP analysis was performed using 220 genes, 194 genes more as compared to the 26 genes of the refined gene list. The relatively mild effects of MBP on the additional genes caused the borderline non-significant deviation of this experimental group. In addition, the significance values of other compounds tested against these Leave-One-Out gene sets showed to be in line with their developmental-toxic properties, with only two exceptions. Altogether, the Leave-One-Out cross validation resulted in correct significance values for track deviations in 27 out of 30 cases, thereby demonstrating the robustness of the approach.

A significant improvement of the 'differentiation track' approach compared to the 'classical EST' is that effects on genes of various differentiation-related processes are included in the analysis, while the 'classical' EST focuses exclusively on inhibition of cardiomyocyte differentiation. In our previous work we showed that MBP and 6-aminonicotinamide, both mainly affecting structures of mesodermal origin, could be identified using the'differentiation track' approach [177]. In the current study, we studied the wider applicability of this approach using compounds that do not only specifically affect mesodermal-derived tissues. RA and VPA mainly affect ectodermal-derived tissues $[186,187]$. Furthermore, also compounds that induce developmental toxic effects via effects on cell survival and proliferation, 5-FU and MAA [97, 98, 184, 185], were included, as well as a negative control (PenG). The identification of differentiation modulation of these diverse developmental toxicants shows that the applicability domain of EST with our new approach is not limited to compounds specifically affecting mesodermalderived tissues.

This study as well as other studies show that gene expression changes are a function of compound concentration $[51,189]$. In the present study developmental toxicants were tested at single concentrations inhibiting cardiomyocyte differentiation. Also in other studies single concentrations were selected that are known to induce effects on classical end points [190, 191]. Auerbach et al. [190] used two concentrations for assessment of carcinogenic activity when evaluating chemicals with unknown carcinogenic potency, which improved the assessment of potential carcinogenicity. Concentrationresponse analysis will be important in future studies to refine our model and to improve the identification of developmental toxicants.

In conclusion, these findings are promising in terms of the prospect of using gene expression profiling in combination with ESC differentiation models in order to predict developmental toxicity. The PCA-derived 'differentiation track' proved to be a suitable concept for use in the dynamic ESC differentiation system, as modulation of gene ex- 
pression correlating with ESC differentiation by developmental toxicants was robustly detected. Further development, standardization and validation of this model are needed via testing of gene-expression modulation of additional compounds in a concentrationresponse design. Additional key genes could be identified in subsequent studies, which may further improve the prediction of developmental toxicity in the model. Enhancing predictivity, throughput and applicability domain are key aspects that may be improved through this approach, facilitating its incorporation as a test system for developmental toxicity in regulatory toxicology.

\section{Acknowledgements}

This work was supported by the Dutch technology society foundation STW [grant MFA6809]. 

Dorien A.M. van Dartel, Jeroen L.A. Pennings,

Liset J.J. de la Fonteyne, Karen J.J. Brauer, Sandra Claessen, Joost H. van Delft, Jos C.S. Kleinjans, Aldert H. Piersma

Toxicological Sciences (2011) 119:126-134 
CHAPTER 6

Evaluation of developmental toxicant identification using gene expression profiling in embryonic stem cell differentiation cultures 


\begin{abstract}
The murine Embryonic Stem cell Test (EST) is an alternative testing method designed to assess potential developmental toxicity of compounds. The implementation of transcriptomic in the EST has been shown to reduce the culture duration and improve end point evaluation, and is expected to result in an enhanced predictability and definition of the applicability domain. We evaluated the identification of developmental toxicity in the EST using two gene sets ('Van_Dartel_heartdiff_24h' and 'EST biomarker genes'), defined in our earlier studies. Non-exposed ESC differentiation cultures were sampled 0,24 , and $48 \mathrm{~h}$ after initiation of differentiation. Additionally, cultures exposed to twelve diverse well-characterized positive and negative developmental toxicants were isolated $24 \mathrm{~h}$ after the onset of exposure. Inhibition of ESC differentiation was evaluated in parallel by morphological scoring on culture day 10. Transcriptomic analysis was conducted using the Affymetrix GeneChips platform. We applied Principal Component Analysis on the basis of the two predefined gene sets to define the 'differentiation track' that represents ESC differentiation. The significance of derivations in the gene expression based 'differentiation track' due to compound exposures were evaluated to determine developmental toxicity of tested compounds. We successfully predicted developmental toxicity using transcriptomics for $83 \%(10 / 12)$ and $67 \%(8 / 12)$ of the compounds, respectively using the two predefined gene sets ('Van_Dartel_heartdiff_24h' and 'EST biomarker genes'). Our study suggests that the application of transcriptomics may improve the applicability of the EST for the prediction of the developmental toxicity of chemicals.
\end{abstract}




\section{Introduction}

Currently, regulatory hazard identification of chemicals is mainly based on animal studies. However, these studies require high numbers of experimental animals, significant amounts of test chemical and have a long duration, which all together make animal studies extremely costly. The need for new approaches has been advocated in a new testing paradigm in toxicology by the US National Research Council (NRC) [192]. Their vision is driven by the need for more efficient tests preferably based on human models, to predict chemical-induced pathway disturbance leading to human disease. The development of cell-line-based models is being encouraged, since such tests can be efficiently designed, which enables high-throughput screening.

One of the best studied cell-line-based tests within developmental toxicology is the Embryonic Stem cell Test (EST) [19], which is therefore of particular interest as model to predict potential developmental toxicity, in line with the view of the NRC. The EST uses murine embryonic stem cells (ESC) that can be induced to differentiate towards contracting cardiomyocytes. Inhibition of this differentiation process by compound exposure can be microscopically observed and is used to evaluate the toxicity of the tested compound. Currently this test is mainly used for prioritization of compounds for further testing in pharmaceutical industry, however, when optimized, the EST may also be used in regulatory settings to predict potential developmental toxicity. Transfer of the assay to a human cell line is a future option.

The aspects of the EST that will need improvement to allow implementation include characterization of the predictability, which is defined by the accuracy of the test model as determined by the percentage of false positives and false negatives. Secondly, the applicability domain of the EST, which pertains to its biological end points as well as the chemical categories for which the test is suitable in terms of prediction [23] has not yet been defined. Finally, also the subjective end point evaluation and the relatively long culture duration are weaknesses of the EST. Together, these aspects are expected to improve by implementation of molecular techniques into the EST.

The first studies that implemented molecular end points into the EST focused on single markers for the prediction of developmental toxicity [25, 30]. Subsequently, Chapin et al. illustrated the usefulness of studying compound-induced regulation of multiple genes and of pathway modulation to identify developmental toxicants [21, 193] as was also suggested by the NRC [192].

For the identification of developmental toxicity using ESC differentiation cultures, we confirmed that gene set-based analyses are highly valuable [111]. We identified 38 genes that were up-regulated in unexposed 24h ESC differentiation cultures, which were combined in a gene set and named 'Van_Dartel_heartdiff_24h'. This novel gene set was uniquely down-regulated in ESC differentiation cultures exposed to the develop- 
mental toxicant monobutyl phthalate, showing its potential in developmental toxicity evaluation. More recently, we developed an alternative tool for developmental toxicant identification in EST by using a continuous representation of ESC differentiation in time using principal component analysis (PCA), defined as the "differentiation track" [177]. A differentiation-track derived gene set was identified, consisting of 26 differentiation-related genes responsive to a group of tested developmental toxicants, which was named 'EST biomarker genes' [194]. In that study, applying a differentiation-track algorithm using ESC differentiation-related gene sets, we successfully identified developmentally toxic compounds.

In the present investigation, we have studied the performance of our gene sets for the identification of developmental toxicity by testing the modulation of ESC differentiation by additional compounds using both the 'Van_Dartel_heartdiff_24h' and the 'EST biomarker genes' gene sets and applying the 'differentiation track' approach. Our analyses show that, with a new and independent data set of compounds tested at single concentrations, the in vitro developmentally toxicity prediction corresponded in $83 \%$ and $67 \%$ with the in vivo data for the 'Van_Dartel_heartdiff_24h' and 'EST biomarker genes' gene sets respectively. These findings suggest that our predictive gene signature approach may be useful in assessing potential developmental toxicity.

\section{Materials and methods}

Test compounds

Test compounds used in this study were monobutyl phthalate (MBP; CAS No. 131-70-4, TCI Europe, Zwijndrecht, Belgium), monomethyl phthalate (MMP; Cas No. 4376-18-5, TCI Europe), monoethylhexyl phthalate (MEHP; CAS No. 4376-20-9, TCI Europe), flusilazole (FLU; Cas No. 85509-19-9, Sigma-Aldrich, Zwijndrecht, The Netherlands), hexaconazole (HEX; CAS No. 79983-71-4, Sigma-Aldrich), triadimefon (TDI; CAS No. 43121-43-3, SigmaAldrich), carbamazepine (CBZ; CAS No. 298-46-4, Sigma-Aldrich), methylmercury chloride (MeHg; CAS No. 115-09-3, Sigma-Aldrich), warfarin (WARF; CAS No. 81-81-2, Fluka, Buchs, Switzerland), nitrofen (NIF; CAS No. 1836-75-5, Fluka), methotrexate (MTX; CAS No. 133073-73-1, Sigma-Aldrich), which were dissolved in dimethyl sulfoxide (DMSO), and saccharine (SACC; CAS No. 82385-42-0, Sigma-Aldrich), which was dissolved in phosphate buffered saline (PBS).

A summary of in vivo developmental effects of the studied compounds is presented in Table 1. 
Table 1. In vivo developmental effects of selected compounds caused by prenatal exposure.

\begin{tabular}{|c|c|c|c|c|}
\hline Compound & LEL & $\begin{array}{l}\text { Target } \\
\text { Species }\end{array}$ & Developmental Toxicity & Literature \\
\hline $\begin{array}{l}\text { Monobutyl } \\
\text { phthalate }\end{array}$ & $250 \mathrm{mg} / \mathrm{kg} \mathrm{bw}$ & Rats & $\begin{array}{l}\text { Male reproductive tract malformations, } \\
\text { cleft palate, cardiovascular, urogenital, } \\
\text { skeletal malformations }\end{array}$ & {$[91,92,198]$} \\
\hline $\begin{array}{l}\text { Monomethyl } \\
\text { phthalate }\end{array}$ & $\geq 1000 \mathrm{mg} / \mathrm{kg} \mathrm{bw}$ & - & - & [121] \\
\hline \multirow[t]{2}{*}{$\begin{array}{l}\text { Monoethylhexyl } \\
\text { phthalate }\end{array}$} & $0.5 \mathrm{mg} / \mathrm{kg} \mathrm{bw}$ & Mice & $\begin{array}{l}\text { Anterior neural tube defects, skeletal } \\
\text { abnormalities }\end{array}$ & [199] \\
\hline & $100 \mathrm{mg} / \mathrm{kg} \mathrm{bw}$ & Rats & $\begin{array}{l}\text { Neural tube defects, vascular } \\
\text { malformations }\end{array}$ & {$[200]$} \\
\hline Flusilazole & $0.4 \mathrm{mg} / \mathrm{kg} \mathrm{bw}$ & Rats & Urogenital malformations & {$[201]$} \\
\hline Hexaconazole & $2.5 \mathrm{mg} / \mathrm{kg} \mathrm{bw}$ & Rats & Skeletal malformations & [201] \\
\hline Triadimefon & $50 \mathrm{mg} / \mathrm{kg}$ bw & Rats & Axial skeletal malformations & [201] \\
\hline \multirow[t]{2}{*}{ Carbamazepine } & $\geq 1000 \mathrm{mg} / \mathrm{kg} \mathrm{bw}$ & Mice & - & {$[202]$} \\
\hline & NR & Human & $\begin{array}{l}\text { Small head circumference, mild facial } \\
\text { dysmorphia, developmental delay }\end{array}$ & {$[202,203]$} \\
\hline $\begin{array}{l}\text { Methylmercury } \\
\text { chloride }\end{array}$ & $6 \mathrm{mg} / \mathrm{kg} \mathrm{bw}$ & Rats & $\begin{array}{l}\text { Delayed vaginal opening, hydrocephalus, } \\
\text { impaired behavioral response }\end{array}$ & {$[204,205]$} \\
\hline \multirow[t]{2}{*}{ Warfarin } & $0.3 \mathrm{mg} / \mathrm{kg} \mathrm{bw}$ & Human & $\begin{array}{l}\text { Nasal hypoplasia, central nervous } \\
\text { disorders }\end{array}$ & [18] \\
\hline & $3 \mathrm{mg} / \mathrm{kg} \mathrm{bw}$ & Rats & Hemorrhage, hydrocephaly & [206] \\
\hline Nitrofen & $0.15 \mathrm{mg} / \mathrm{kg} \mathrm{bw}$ & Rats & $\begin{array}{l}\text { Diaphragmatic hernia, abnormal } \\
\text { development of heart, kidney, lung }\end{array}$ & {$[207]$} \\
\hline Methotrexate & $0.3 \mathrm{mg} / \mathrm{kg} \mathrm{bw}$ & Rats & $\begin{array}{l}\text { Embryolethal, cleft palate, skull defects, } \\
\text { and severe fore and hindlimb dysplasias }\end{array}$ & [208] \\
\hline Saccharine & $\geq 1000 \mathrm{mg} / \mathrm{kg} \mathrm{bw}$ & - & - & {$[90]$} \\
\hline
\end{tabular}

LEL= Lowest Effect Level, NR=Not Reported

Resazurin cell viability assay

The effect of compound exposure on cell number was determined using resazurin dye reduction, which is a measure for the number of viable cells per well [178], as we described previously [150]. For each compound a minimum of five concentrations were tested in three independent experiments. Cultures were exposed to a maximum DMSO concentration of $0.2 \%$, which did not affect cell viability. The resazurin reduction of exposed cells relative to control measurement was calculated and plotted against the test concentrations using PROAST software [103]. The cell survival at the concentrations tested in the transcriptomic experiment was calculated on the basis of the curve fit. 
Pluripotent embryonic stem cell culture and cardiomyocyte differentiation

Pluripotent murine D3 embryonic stem cells (ESC; ATCC, Rockville, MD) were cultured in a monolayer in Dulbecco's Modified Eagle Medium (DMEM) based (Gibco BRL, Gaithersburg, MD) medium supplemented with leukemia inhibitory factor (LIF; Chemicon, Temecula, CA), as described previously [7, 102]. In short, hanging drop cultures were set up to obtain embryoid bodies (EB) using single ESC suspensions in complete medium without LIF. [7, 102, 177]. These EB cultures were used as the primary step for differentiation of ESC. Inhibition of cardiomyocyte differentiation was evaluated by calculating the mean scores of 24 differentiation cultures relative to the mean scores of control cultures. In total four classes of differentiation were defined: Strongly beating differentiation cultures (multiple contracting foci and $>40 \%$ of the cells beating) received three points, moderately beating cultures (one or more contracting foci and $10-40 \%$ of the cells beating) was given a score of two, weakly beating differentiation cultures (one contracting focus, $<10 \%$ of the cells beating) received only one point. Differentiation cultures without presence of contracting cardiomyocyte foci were scored negative and received 0 points.

Inhibition of cardiomyocyte differentiation by compound exposure

ESC differentiation cultures were exposed from the EB-stage at day three onwards to effective concentrations of the selected test compounds, that were calculated (on the basis of historic data) to inhibit ESC differentiation by $50 \%\left(\mathrm{ID}_{50}\right)$ using PROAST curve fitting [103]. All cultures, including control non-exposed cultures, were exposed to noncytotoxic $0.2 \%$ DMSO concentrations for comparability reasons. Cultures were microscopically evaluated at day 10 of the culture $(n=2)$ to assess the inhibition of cardiomyocyte differentiation, while parallel cultures were used for gene expression analysis at earlier time points as explained below.

RNA isolation and whole genome gene expression profiling

RNA of compound-exposed cultures was isolated $24 \mathrm{~h}$ after the onset of exposure, which corresponds with culture day 4. DMSO-exposed control differentiation cultures were harvested after 0, 24 and $48 \mathrm{~h}$ from the EB-stage at day 3, which corresponds with culture day 3, 4, and 5. Each experimental group consisted of 8 replicates of which the cells were directly collected in RNA protect (Qiagen, Venlo, The Netherlands) to stabilize RNA. Total RNA was purified using the RNeasy plus mini kit (Qiagen, Venlo, The Netherlands) according to the manufacturer's instructions. The quantity of RNA in each sample was measured using a NanoDrop Spectrophotometer (NanoDrop technologies, Wilmington, DE) and RNA integrity was assessed on the 2100 Bioanalyzer (Agilent Technologies, Amstelveen, The Netherlands) using the RNA 6000 Nano Chip Kit (Agilent Technologies). Preparation of the RNA samples for gene expression analysis using the Mouse Genome 
4302.0 arrays (Affymetrix) was done as described previously [194]. Quality controls were performed according to the manufacturer's instructions and were within acceptable limits for all arrays.

Data analysis and statistics

Robust Multichip Average (RMA) normalization of Affymetrix CEL files and probe to gene mapping were performed as we described previously [194]. Of the probe-set definitions included in the custom annotation, 16,331 probe sets defined by the Brainarray custom Chip Description File (CDF) version 11 (http://brainarray.mbni.med.umich.edu/Brainarray/Database/CustomCDF) [182] and 4648 additional probe sets defined by Affymetrix chip annotation 26 were used in further analyses, giving a total of 20,979 probe sets. Probe sets for Affymetrix internal controls or probe sets that did not correspond to an Entrez Gene ID were not used in further analyses. Raw and normalized data are available from ArrayExpress (http://www.ebi.ac.uk/arrayexpress/) under accession number E-MTAB-300.

Statistical analyses were carried out using the $\mathrm{R}$ statistical software environment (http://www.r-project.org) using log-transformed values. Maximal fold ratios (FR) in individual gene expression between the experimental groups were determined by comparing the average normalized signal values per group and were calculated as the maximum/minimum ratio. Genes that were significantly differentially expressed between any of the non-exposed experimental groups were identified by a one-way ANOVA on the normalized data, using the cut-off criteria of a $p<0.001$ and $F R>1.5$. The percentage of commonly regulated genes was calculated relative to the limiting gene set.

Definition of the differentiation track

To visualize normal ESC differentiation, we defined the 'differentiation track' of these cultures as we described previously [177]. In short, PCA analysis was performed with R using defined gene lists that visualize the continuous differentiation of ESC on the basis of gene expression dynamics that represent the differentiation track. Coordinates along the first and second principal component were calculated for each sample and deviation of compound exposed cultures from the 'differentiation track' was analyzed by applying a Hotelling T-test to these coordinates compared to those of time-matched control cultures. Significant deviation from the 'differentiation track' $(p<0.05)$ was considered characteristic for developmental toxicants.

To study the correlation between the degree of differentiation inhibition and the significance of 'differentiation track' deviation, Spearman's rank correlation coefficient was calculated. 

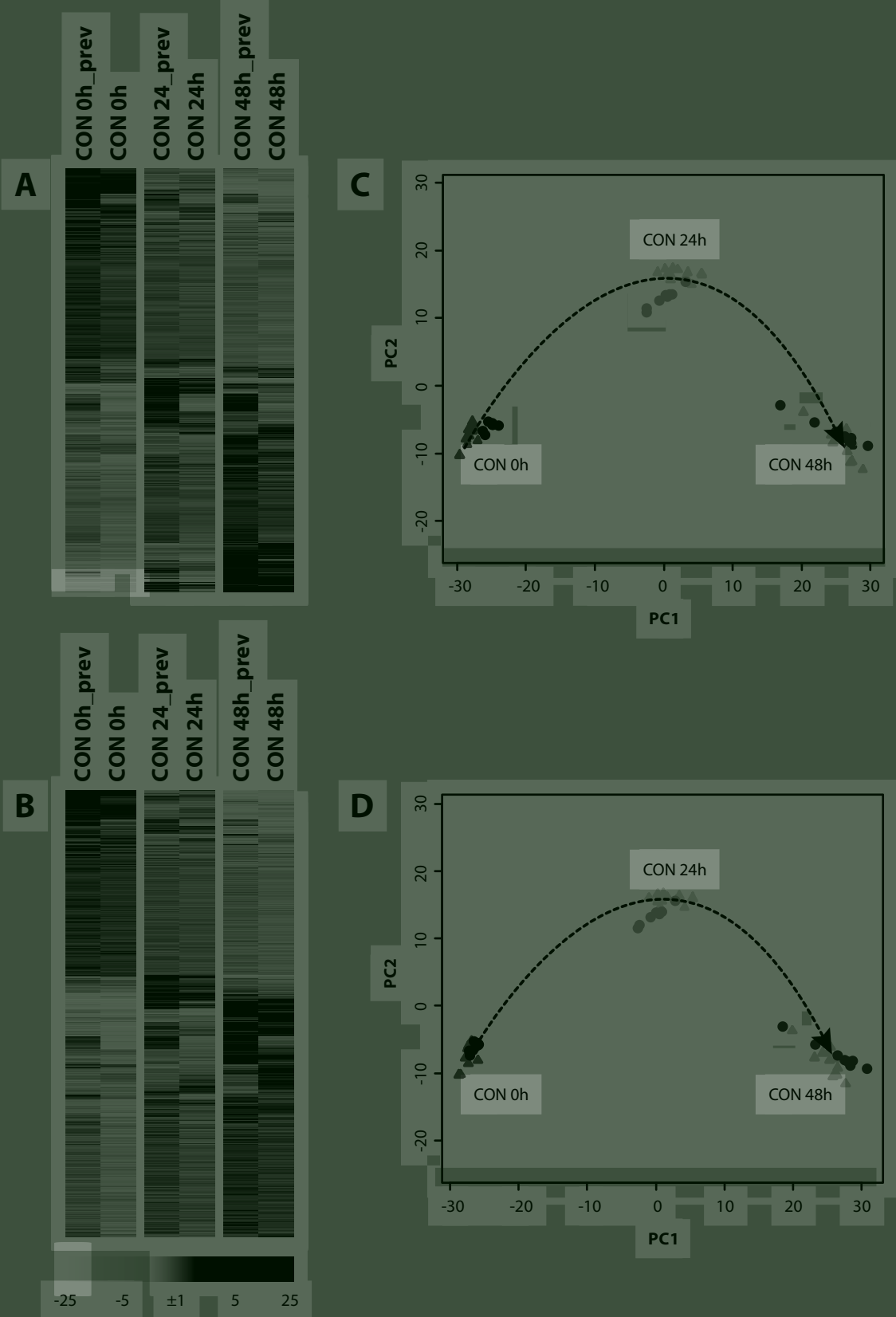

Figure 1. Heatmap of the mean gene expression values of the unexposed control samples at 0, 24, and $48 \mathrm{~h}$ of differentiation in our previous (_prev; van Dartel et al., 2010a) and present study using the differentiated-related genes identified in our previous study (3579 genes; $A$ ) and in the present study (3780 genes, B). PCA plots based on ESC differention-related genes in our previous study (C) and the present study (D). Unexposed ESC differentiation samples of the previous study (circles) and the current study (triangles) are presented. For color figure, see Color figures section. 
Table 2. Commonly regulated genes in ESC differentiation studies.

\begin{tabular}{lll}
\hline & $\begin{array}{l}\text { Present study FR>1.5 } \\
(\mathbf{n = 3 7 8 0})\end{array}$ & $\begin{array}{l}\text { Present study FR>1 } \\
(\mathbf{n = 9 7 7 4 )}\end{array}$ \\
\hline Previous study $^{\mathrm{a}} \mathrm{FR}>1.5(\mathrm{n}=3579)$ & $2700(71.4 \%)$ & $3278(91.5 \%)$ \\
Previous study $^{\mathrm{a}} \mathrm{FR}>1(\mathrm{n}=12409)$ & $3658(96.8 \%)$ & $8613(88.1 \%)$ \\
\hline
\end{tabular}

a van Dartel et al., 2010a

\section{Results}

ESC differentiation-related gene regulation

The gene expression profiles of the unexposed ESC differentiation samples at 0, 24 and $48 \mathrm{~h}$ were compared revealing 3780 genes that were transcriptionally responsive to ESC differentiation, using $p<0.001$ (corresponding false discovery rate $=0.0021$ ) and $F R>1.5$. We compared these genes to the 3579 genes that we identified previously as ESC differentiation-related genes, using the same experimental design [177]. In total, a large number of genes ( 2700 genes, $75.4 \%$ ) were identified to be commonly regulated in both studies and showed similar differentiation dynamics (Figure 1). Using $p<0.001$ and $F R>1.0$ for the present study, this overlap between differentiation-regulated genes was $91.5 \%$, indicating that most of the non-overlapping genes were still significantly regulated in the present study, but to a lesser extent (Table 2).

The unexposed experimental samples of this study and of our previous study were plotted using Principal Component Analysis (PCA) using the genes that were identified as differentiation-related genes in the previous study (3579 genes, Figure 1C) and in the present study (3780 genes, Figure 1D). Replicates within experimental groups clustered together, as well as experimental groups isolated after the same culture duration in the two different studies. Furthermore, the PCA-plot showed that experimental groups appeared in chronological order and continuous ESC differentiation could therefore be represented by the curve connecting these samples, which we named 'the differentiation track'. The position of the time-matched experimental groups of our present study (triangles) compared to our previous study (circles) showed some variation, which was most likely caused by slightly different temporal progression of ESC differentiation between experiments.

Compound-induced effect on ESC differentiation and cellular viability

ESC differentiation cultures were exposed to single test concentrations, selected on the basis of historic data. Effects of the test compounds on ESC differentiation and cell viability were scored in cultures parallel to those analyzed for gene expression in the present study, and results are presented in Figure 2. SACC and MMP did not affect ESC differentiation at high concentrations tested, which is in line with their absence of ef- 


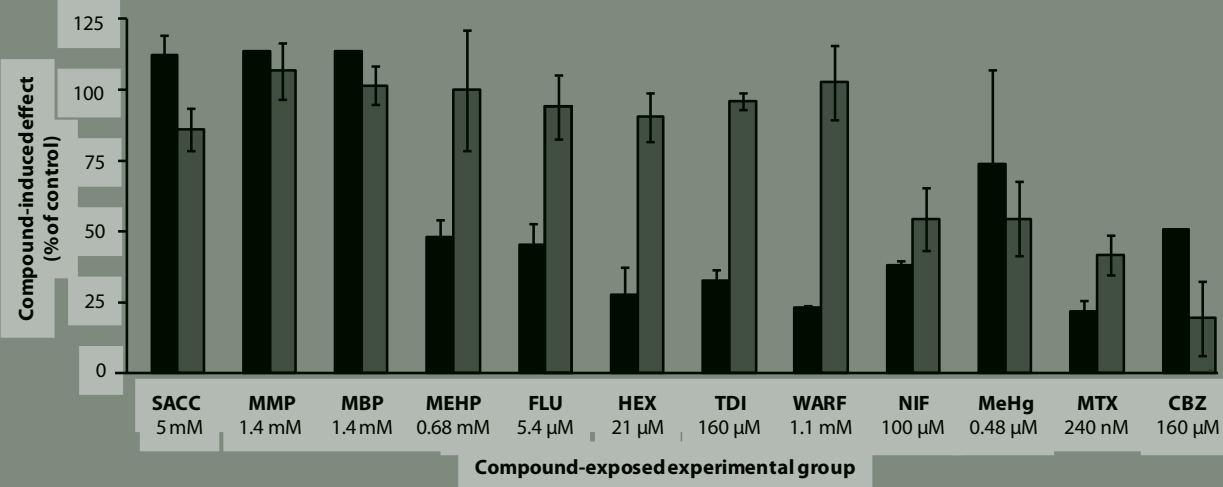

Figure 2. Average ESC differentiation ( $n=2$, error bars indicate upper and lower values) and average cell viability ( $n=3$, error bars indicate standard deviation) as observed in the compound-exposed cultures that were cultured in parallel to those tested for gene expression.

fects on embryonic development. Only mild effects on cell viability were observed in SACC-exposed cultures ( $85 \%$ cell viability). No effects on cell viability and, remarkably, also no effects on ESC differentiation were observed in the cultures exposed to the developmentally toxic compound MBP. On the other hand, the in vivo more potent phthalate MEHP, and also FLU, HEX, TDI and WARF affected ESC differentiation effectively in absence of cytotoxicity or proliferation inhibition ( $>90 \%$ cell viability). Cultures exposed to NIF, MeHg, MTX and CBZ also inhibited ESC differentiation, but these compounds also induced a moderate to strong reduction of cell number, ranging from 19\% in CBZexposed cultures to $54 \%$ in MeHg-exposed cultures.

Compound-induced deviation from the differentiation track

To study inhibition of ESC differentiation by compound exposure, we applied the 'differentiation track' algorithm using the predefined differentiation-related gene sets 'Van_Dartel_Heartdiff_24h' and 'EST biomarker genes'. The majority of the genes within these sets were also differentially expressed among ESC differentiation cultures across time in the present study, namely 32/38 of the 'Van_Dartel_heartdiff_24h' and 22/26 of the 'EST biomarker genes' gene set (Figure 3A and B). These predefined gene sets represent slightly different ESC differentiation dynamics as reflected by the differences in the projections of the differentiation tracks in the PCA plots. Both gene sets could successfully be used to describe the 'differentiation track' as shown in Figure 3C and D.

The modulation of ESC differentiation was analyzed by calculating the significance of deviation of exposed cultures from control ESC differentiation using the two predefined gene sets 'Van_Dartel_heartdiff_24h' and 'EST biomarker genes' (Table 3A). The nonsignificant deviation of SACC using the 'Van_Dartel_heartdiff_24h' gene set and the 

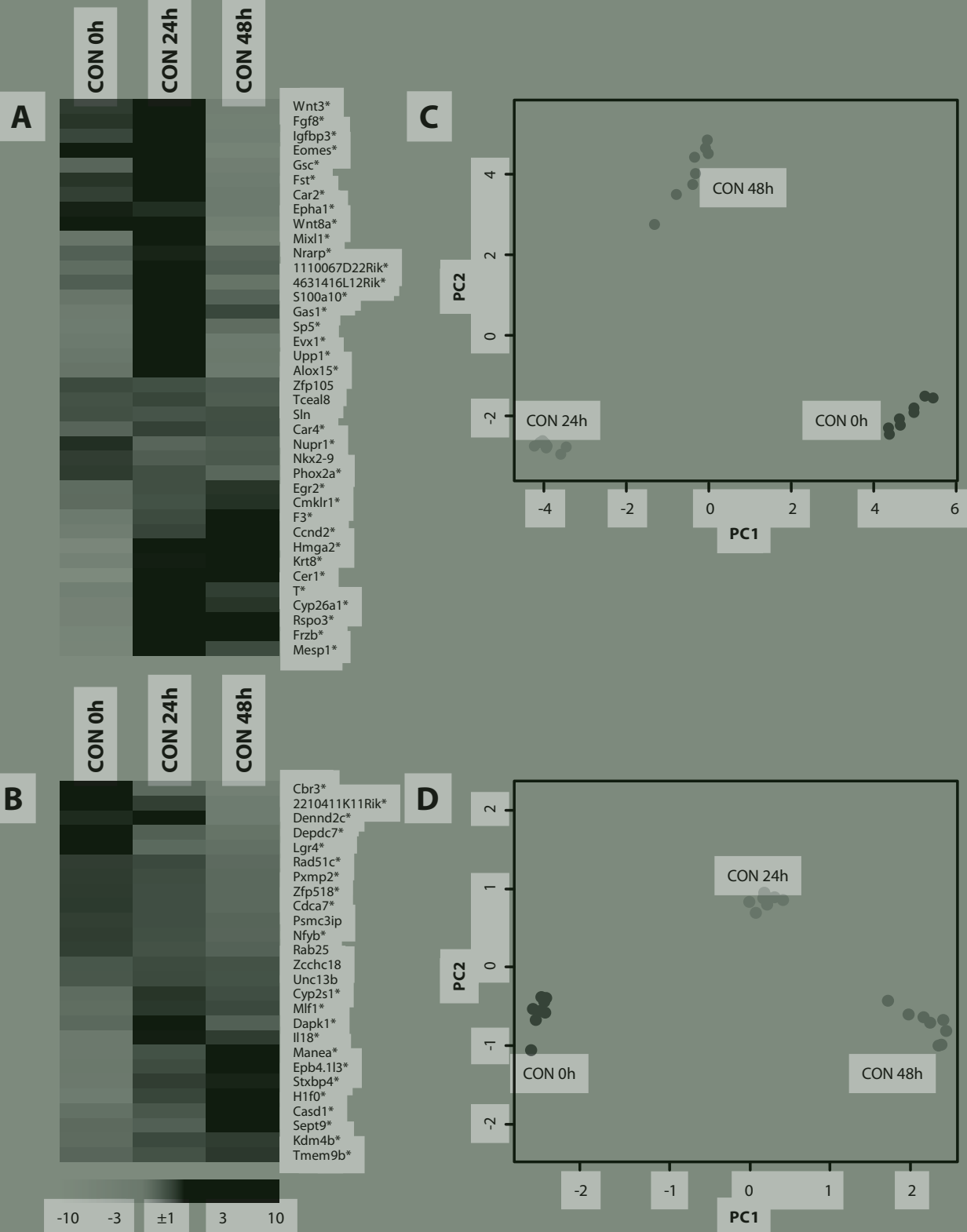

Figure 3. Mean gene expression of the 'Van_Dartel_heartdiff_24h' (A) and 'EST biomarker genes' (B) gene sets at $0 \mathrm{~h}, 24 \mathrm{~h}$, and $48 \mathrm{~h}$ of control ESC differentiation cultures (CON). * Indicate significantly differentially expressed genes among the $0 \mathrm{~h}, 24 \mathrm{~h}$, and $48 \mathrm{~h}$ CON groups. Principal Component Analysis of the control ESC differentiation cultures (individual samples, circles) at $0 \mathrm{~h}, 24 \mathrm{~h}$, and $48 \mathrm{~h}$ of ESC differentiation using the 'Van_Dartel_heartdiff_24h' (C) and 'EST biomarker genes' (D). For color figure, see Color figures section. 
Table 3. Significance of deviation of compound-exposed cultures of the present study $(A)$ and our previous study (B; van Dartel et al., 2010a) from the defined 'differentiation track' ( $p$-value) using selected gene sets. *Statistical significant deviation of compound-exposed from time-matched cultures on the 'differentiation track' $(p<0.05)$. Significance values that correspond with the in vivo developmental toxicity of the compound are in bold.

A

\begin{tabular}{|c|c|c|}
\hline $\begin{array}{l}\text { Compound-exposed } \\
\text { experimental group }\end{array}$ & $\begin{array}{l}\text { 'Van_Dartel_Heartdiff_24h'a } \\
\text { ( } n=38 \text { genes) }\end{array}$ & $\begin{array}{l}\text { 'EST Biomarker genes' }(n=26 \\
\text { genes) }\end{array}$ \\
\hline SACC & 0.059 & $2.1 \mathrm{E}-2^{*}$ \\
\hline MMP & 0.159 & 0.072 \\
\hline MBP & 3.7E-2* & 0.061 \\
\hline MEHP & 4.5E-3* & $1.2 \mathrm{E}-3^{*}$ \\
\hline FLU & $2.5 \mathrm{E}-3^{*}$ & 3.7E-2* \\
\hline HEX & 0.053 & $2.0 \mathrm{E}-2^{*}$ \\
\hline TDI & $5.6 \mathrm{E}-3^{*}$ & $7.8 \mathrm{E}-3^{*}$ \\
\hline WARF & $6.3 \mathrm{E}-09^{*}$ & $5.7 E-3^{*}$ \\
\hline NIF & 0.271 & 0.229 \\
\hline $\mathrm{MeHg}$ & $4.8 \mathrm{E}-2^{*}$ & 0.18 \\
\hline MTX & $1.2 \mathrm{E}-05^{*}$ & $2.3 E-3^{*}$ \\
\hline CBZ & $9.1 \mathrm{E}-3^{*}$ & $1.1 \mathrm{E}-3^{*}$ \\
\hline
\end{tabular}

B

\begin{tabular}{ll}
\hline $\begin{array}{l}\text { Compound-exposed } \\
\text { experimental group }\end{array}$ & $\begin{array}{l}\text { 'Van_Dartel_Heartdiff_24h' } \\
\text { ( } \mathbf{n}=\mathbf{3 8} \text { genes) }\end{array}$ \\
\hline Penicillin G & $\mathbf{0 . 1 1 8}$ \\
5-fluorouracil & $\mathbf{1 . 0 E - 1 0 ^ { * }}$ \\
methoxyacetic acid & $\mathbf{2 . 1 E - 7 ^ { * }}$ \\
monobutylphthalate & 0.186 \\
retinoic acid & $\mathbf{4 . 1 E - 6 ^ { * }}$ \\
valproic acid & $\mathbf{4 . 0 E - \mathbf { 2 } ^ { * }}$ \\
\hline
\end{tabular}

a van Dartel et al., 2009a

${ }^{\mathrm{b}}$ van Dartel et al., $2010^{\mathrm{a}}$

non-significant deviation of MMP with both gene sets were in line with the in vivo nondevelopmentally toxic properties of these compounds. The majority of the ten positive developmental toxicants deviated significantly from the time-matched unexposed cultures on the differentiation track, which was expected on the basis of their in vivo effects. MBP, HEX and MeHg were falsely predicted as being non-developmentally toxic in one out of two predictions, although these $p$-values were borderline non-significant for MBP $(p=0.061)$ and HEX ( $p$-value=0.053). The developmental toxicant NIF was consistently incorrectly predicted as a negative developmentally toxic compound using both gene sets. We also evaluated the performance of the 'Van_Dartel_heartdiff_24h' gene set 
Table 4. Classification results of the 12 compounds tested in the present study using the 'Van_Dartel_ heartdiff_24h' gene set (A) and 'EST biomarker genes' gene set (B), and of the six compounds tested in our previous study (van Dartel et al., 2010a) using the 'Van_Dartel_heartdiff_24h' gene set (C).

A

\begin{tabular}{lccc}
\hline 'Van_Dartel_heartdiff_24h a & \multicolumn{2}{c}{ In vivo embryotoxicity classification } \\
\hline In vitro embryotoxicity prediction & Positive & Positive & Negative \\
\hline & Negative & 8 & 0 \\
\hline B & & 2 & 2 \\
\hline 'EST biomarker genes'b & & In vivo embryotoxicity classification \\
\hline & & Positive & Negative \\
\hline In vitro embryotoxicity prediction & Positive & 7 & 1 \\
& Negative & 3 & 1 \\
\hline C & & & \\
\hline 'Van_Dartel_heartdiff_24h'a & & In vivo embryotoxicity classification \\
\hline & & Positive & Negative \\
\hline In vitro embryotoxicity prediction & Positive & 4 & 0 \\
& Negative & 1 & 1 \\
\hline
\end{tabular}

a van Dartel et al., 2009a

${ }^{\mathrm{b}}$ van Dartel et al., $2010^{\mathrm{a}}$

for the identification of developmental toxicity using data of our previous study [194] (Table 3B). Five out of the six compounds were correctly identified, using this gene set.

An overview of the predictions of the compounds tested in this study using the 'differentiation track' approach with the gene sets 'Van_Dartel_heartdiff_24h' and 'EST biomarker genes' is shown in Table 4A and 4B, respectively. The overall accuracy of the predictions using the 'Van_Dartel_heartdiff_24h' gene set was 83\% (10/12). The two incorrect predicted compounds were positive developmental toxicants that were predicted to be negative (HEX and NIF, Table 3). The accuracy of the predictions using the 'EST biomarker genes' was $67 \%$, since 8 out of 12 predictions were correctly predicted. The falsely predicted compounds included one negative compound (SACC) and three positive compounds (MBP, NIF, MeHg, Table 3). Evaluation of the 'Van_Dartel_heartdiff_24h'gene set using data of our previous study [194] showed that 83\% (5/6) of the compounds were correctly identified (Table 4C). MBP appeared to be the only misclassified compound, being falsely labeled as non-developmentally toxic.

Spearman correlation coefficients ( $R$ values) were calculated of the correlation between the significance of 'differentiation track' deviation and the degree of ESC differentiation inhibition based on available data from our studies. The $R$ values, -0.71 and -0.69, obtained for the 'Van_Dartel_heartdiff_24h' and 'EST biomarker genes' respectively, revealed that the significance of deviation from the 'differentiation track' is 
partly dependent on the degree of ESC differentiation inhibition (Figure 4). Therefore, for correct prediction of developmental toxicity, the effect size of the concentration of the test compound used appears to be an important factor.

\section{Discussion}

Reproducible gene responses in ESC differentiation

The present study showed that gene expression signatures in ESC differentiation are highly reproducible between independently performed studies. As many as $91.5 \%$ of the previously identified differentiation-related genes [194] were identified as significantly regulated in the present study.

We performed PCA analysis to visualize the continuous differentiation of ESC on the basis of gene expression dynamics that represent the differentiation track. PCA has been shown previously to be a valuable approach to describe embryonic development [153], neural cell lineage differentiation [195], and ESC differentiation [177]. In the present study, the differentiation tracks on the basis of the genes identified to be involved in ESC differentiation in both studies were very similar (Figure 1C versus 1D). Furthermore, the differentiation tracks showed that the samples of the present study differed only slightly from the time-matched samples of our previous study. The variation was observed along the track, and therefore this difference may be primarily explained by a slight variation in the timing of differentiation. In addition, also within experimental groups, variation of ESC differentiation was observed along the differentiation track, supporting the notion that the track represents continuous ESC differentiation.

The reproducibility of gene expression profiles of differentiating ESC facilitates the use of a predefined gene set for developmental toxicity prediction. Apparent variance in temporal progression of ESC differentiation between experiments is not expected to hamper the use of predefined gene sets, since the genes within the gene sets also showed temporal regulation and thus follow the dynamics of ESC differentiation.

Differentiation track-based evaluation of developmental toxicity

Studying gene expression signature in differentiation ESC allows the possibility to investigate compound-induced effects on multiple cellular processes. The present study shows that compounds that affected development either via proliferation or differentiation were detected in the EST protocol. Although effects on the mesodermal-derived cardiomyocyte differentiation has been classically used as the end point for evaluation of differentiation inhibition, also compounds affecting structures derived from other germ layers can potentially be discriminated using the EST [19]. This is explained by the presence of cells from the ectodermal and endodermal germ layers within the differentiation 
cultures $[6,87]$, which resembles the in vivo situation, in which the mesodermal germ layer develops by induction between the ectoderm and endoderm germ layers [113].

The predictive capacity of both gene sets appears promising in view of the accuracies that were calculated using data of a limited set of 12 test compounds tested at single concentrations. The accuracies obtained were $83 \%$ for the gene set 'Van_Dartel_heartdiff_24h' and 67\% for the gene set 'EST biomarker genes'. Unfortunately, the negative control SACC was misclassified using the 'EST biomarker genes' gene set. This could be explained by the cell viability results, which showed that SACC caused some degree of cytotoxicity at the concentration tested in the transcriptomic study. Regard-

Van_Dartel_Heartdiff_24h', $R=-0.71$

$$
\text { }
$$

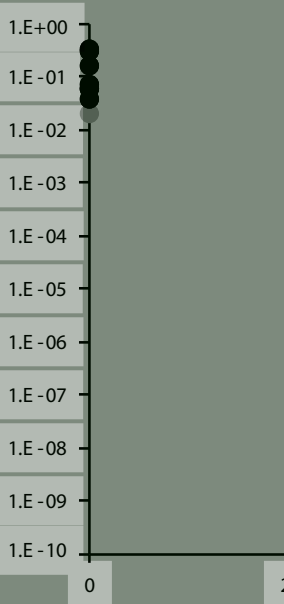

EST biomarker genes', $R=-0.69$

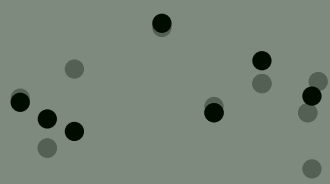

Figure 4. Spearman correlation of significance of deviation from the 'differentiation track' using 'EST biomarker genes' and 'Van_Dartel_heartdiff_24h' with ESC differentiation inhibition.

ing the false-negative compounds, two of which were observed using the 'Van_Dartel_heartdiff_24h'set (HEX and NIF), and three using the 'EST biomarker genes' set (MBP, $\mathrm{NIF}, \mathrm{MeHg}$ ), two predictions appeared to be borderline non-significant (HEX and MBP) and one incorrect prediction may be caused by the observed large variation in ESC differentiation inhibition ( $\mathrm{MeHg}$, Figure 2). Data analyses of our previous transcriptomic study showed that MBP was also incorrectly identified as being non-developmentally toxic using the 'Van_Dartel_heartdiff_24h' gene set. The significance values for 'differentiation track' deviation correlated well with limited differentiation inhibition (Figure 4) and this may explain the misclassifications of MBP and MeHg.

Remarkably, although the strong developmental toxicant NIF inhibited ESC differentiation as assessed on day 10 of the EST protocol, NIF was not identified as develop- 
mental toxicant using the 'differentiation track' method. The regulation of 570 genes by NIF (not shown), included many genes that are known to play an important role in development, such as Hoxd9, Wnt8a, Tdgf1 and Shh. Regarding the significant number of NIF-regulated genes, it is not likely that the timing for studying gene expression was insufficient. Apparently, the effect of NIF was not reflected by the gene sets that were used for studying differentiation modulation. The gene sets used in the present study, Van_Dartel_heartdiff_24h' and 'EST biomarker genes', will need further refinement to optimize identification of a wider array of developmental toxicants and to assess their relative validity.

Aspects for further optimization of developmental toxicant identification in the EST

Obviously, compound concentration is an important factor when assessing the toxicity of compounds, as has been shown also in the EST $[150,196,197]$. In the present study, the identification of developmentally toxic compounds was based on gene expression data of compounds tested at single concentrations only, which represents the most economic gene array study design. With this approach we were able to show that the degree of ESC differentiation inhibition correlated well with the significance of 'differentiation track' deviation. Previous studies have shown that also the gene expression response is related to compound concentration [51, 189]. Therefore, the prediction of developmental toxicants is expected to improve by analyzing transcriptomic responses of test compounds in a concentration-response design, and although significantly more cost-intensive, this will be the subject of our future studies.

Additional improvements of our approach will include increasing the throughput of the assay using custom gene chips. In addition, future studies may be based on compound-induced gene signatures of human ESC (hESC). Implementation of these hESC is expected to improve the predictability of the assay [16]. Unfortunately, routine hESC culture as well as hESC differentiation protocols are still very labor-intensive and therefore the development of optimized culture protocols is essential for successful use of hESC in the EST.

Conclusions

In this transcriptomic study, we have demonstrated the application of gene expression analysis for the prediction of potential developmental toxicity, by testing compounds at single concentrations. To our knowledge this is the first study that evaluates this approach in the field of developmental toxicology. This approach may lead to improvements of the predictability and the characterization of the applicability domain of the EST, which may ultimately facilitate its incorporation as a test system for developmental toxicity in regulatory strategies. 


\section{Acknowledgements}

This work was supported by the Dutch technology society foundation STW [grant MFA6809]. The sponsor was not involved in analysis or interpretation of the data. The authors declare that they have no competing financial interests. 
Dorien A.M. van Dartel, Jeroen L.A. Pennings,

Liset J.J. de la Fonteyne, Karen J.J. Brauers, Sandra Claessen, Joost H. van Delft, Jos C.S. Kleinjans, Aldert H. Piersma

Toxicology and Applied Pharmacology (2011) 251:110-118 
CHAPTER 7

Concentration-dependent gene expression responses to flusilazole in embryonic stem cell differentiation cultures 


\begin{abstract}
The murine Embryonic Stem cell Test (EST) is designed to evaluate developmental toxicity based on compound-induced inhibition of embryonic stem cell (ESC) differentiation into cardiomyocytes. The addition of transcriptomic evaluation within the EST may result in enhanced predictability and improved characterization of the applicability domain, therefore improving usage of the EST for regulatory testing strategies. Transcriptomic analyses assessing factors critical for risk assessment (i.e. concentration) are needed to determine the value of transcriptomic evaluation in the EST. Here, using the developmentally toxic compound, flusilazole, we investigated the effect of compound concentration on gene expression regulation and toxicity prediction in ESC differentiation cultures. Cultures were exposed for $24 \mathrm{~h}$ to multiple concentrations of flusilazole $(0.54-54 \mu \mathrm{M})$ and RNA was isolated. In addition, we sampled control cultures 0,24 , and $48 \mathrm{~h}$ to evaluate the transcriptomic status of the cultures across differentiation. Transcriptomic profiling identified a higher sensitivity of development-related processes as compared to cell division-related processes in flusilazole-exposed differentiation cultures. Furthermore, the sterol synthesis-related mode of action of flusilazole toxicity was detected. Principal Component Analysis using gene sets related to normal ESC differentiation was used to describe the dynamics of ESC differentiation, defined as the 'differentiation track'. The concentration-dependent effects on development were reflected in the significance of deviation of flusilazole-exposed cultures from this transcriptomic-based differentiation track. Thus, the detection of developmental toxicity in EST using transcriptomics was shown to be compound concentration-dependent. This study provides further insight into the possible application of transcriptomics in the EST as an improved alternative model system for developmental toxicity testing.
\end{abstract}




\section{Introduction}

The current regulatory guidelines for toxicological hazard identification require large numbers of experimental animals. As a consequence of the implementation of the new EU regulation for Registration, Evaluation and Authorization of Chemicals (REACH) in 2007, the potential toxicity of thousands of chemicals needs to be assessed over the coming years. To reduce the number of experimental animals needed within REACH, alternative methods for toxicity testing are required. Since more than $60 \%$ of experimental animals in REACH will be used for reproductive and developmental toxicity assessments [119], the demand for in vitro alternative test methods is particularly high for these areas of testing. Several alternative methods for the identification of developmental toxicity have been developed, varying from whole embryo cultures of rat or zebrafish, and organ cultures, to continuous cell-line based assays, such as the Embryonic Stem cell Test (EST) $[19,84-86]$. None of these assays have achieved regulatory acceptance, due in part to their uncertain predictive capacity as well as their ill-defined applicability domain, i.e. the biological end points that are incorporated and the chemical categories for which developmental toxicity can correctly be predicted.

The EST is a cell-line based standardized in vitro assay that has been established to classify compounds with respect to their developmentally toxic potential [19]. The EST measures the inhibition of murine ESC differentiation into contracting cardiomyocytes upon exposure to compounds. The EST is considered a promising alternative assay for developmental toxicity prediction [170]. However, it still has several weaknesses that need improvement to allow successful regulatory acceptation. These aspects include the relatively long culture duration, subjective and laborious end point scoring and the limited characterization of its applicability domain and predictability [170].

Since the emergence of transcriptomics in the 1990s [209], many studies have shown that this technique provides a powerful tool to study toxicological mechanisms and toxicity profiles at the level of gene expression. The use of transcriptomics in predictive toxicology has been extensively studied for the prediction of carcinogenicity, using liver tissue of in vivo exposed experimental animals [33, 34, 36, 37, 210]. These data indicate that a gene expression-based predictive model can be an effective tool for identifying carcinogens. In addition, promising results have been published in other fields of toxicology, including developmental toxicology [41].

The implementation of transcriptomics in the EST may validate this test as a successful in vitro model for predicting developmental toxicity. We recently showed that compound-induced gene regulation could be observed shortly after the initiation of compound exposure, resulting in an abbreviated test protocol [111, 177]. Furthermore, gene expression changes pertain not to cardiac muscle cells only but to all cell types present in the culture. Therefore, gene expression end points may improve the predict- 
ability and applicability domain of the model. We have identified ESC differentiationrelated genes and showed that developmental toxicants could be identified by the differential expression of these genes [194].

So far, most transcriptomic studies have investigated the toxicity of a single test compound concentration. Only few studies have addressed concentration-dependent gene expression changes, often by testing only a low and a high dose [51, 211], but also more comprehensive concentration-response studies have been described [49, 212, 213]. Mostly, the selection of these concentrations has been based on their observed phenotypic/morphological effects in the model system. Overall, compound concentration appeared to relate to both the magnitude of gene induction and the number of differentially expressed genes. Several studies have shown that dose-response assessment of gene expression is of great importance for correct prediction of toxic properties $[41,49,75,190]$. In a recent study, we showed the interdependence of morphological effect size and the extent of gene expression changes, suggesting that effect prediction through gene expression is modulated by compound concentration [214].

The present study was designed to investigate the effect of compound concentration on gene expression in ESC differentiation cultures. Six concentrations of flusilazole were tested, which makes this study one of the most extensive studies in concentrationrelated gene expression responses to date. Flusilazole was selected due to its known developmental toxic properties, causing growth retardation and skeletal abnormalities in rodent models [215] and its relevance for human health risk in view of its global use as a fungicide in agricultural practice [216]. In the present study, gene expression profiling confirmed concentration-dependent transcriptomic changes in differentiating ESC exposed to flusilazole. Gene expression-linked developmental-related processes appeared to be most sensitive to flusilazole exposure. Genes related to cell division as well as processes related to sterol metabolism, the pharmacological mode of action of flusilazole, were found to be affected. Furthermore, we applied Principal Component Analysis (PCA) using gene sets related to ESC differentiation to describe the dynamics of differentiating ESC in the EST protocol, previously defined as the 'differentiation track' [177]. These analyses showed that the concentration-dependent effects on development were also evident by the extent of deviation of flusilazole-exposed cultures from the differentiation track. This study provides further insight into the use of transcriptomics in ESC differentiation as a model system for developmental toxicology. 


\section{Materials and methods}

Pluripotent embryonic stem cell culture and differentiation

Pluripotent murine D3 embryonic stem cells (ESC; ATCC, Rockville, MD) were cultured in monolayer in Dulbecco's Modified Eagle Medium (DMEM) based (Gibco BRL, Gaithersburg, MD) medium supplemented with leukemia inhibitory factor (LIF; Chemicon, Temecula, CA), as described previously [7]. Induction of cardiomyocyte differentiation was performed according to the INVITTOX protocol [7] with few adaptations [102, 177].

Resazurin cell viability assay

To facilitate interpretation of the gene expression data, we assessed the effect of flusilazole (CAS No. 85509-19-9, Sigma-Aldrich, Zwijndrecht, The Netherlands) exposure on cell viability. We used resazurin dye reduction as a measure for the number of viable cells, as described previously [150]. Flusilazole was dissolved in dimethyl sulfoxide (DMSO) and ESC differentiation cultures were exposed to final DMSO concentrations of $0.2 \%$, which did not affect cell viability. Control cultures were also exposed to $0.2 \%$ DMSO for comparability reasons. ESC differentiation cultures were exposed to flusilazole during the first 3 days of the EST protocol, since effects on cell viability are most pronounced during this early phase of the EST protocol [150]. Flusilazole was tested at concentrations ranging from 0.1 to $300 \mu \mathrm{M}$ in three independent runs. The resazurin reduction of exposed cultures relative to solvent control cultures was calculated and plotted against the flusilazole concentrations using PROAST software [103]. The cell survival at the concentrations tested in the transcriptomic experiment and the concentration at which cell viability is reduced by $50 \%$ (IC50) were calculated on the basis of the curve fit.

Inhibition of ESC differentiation by flusilazole exposure

Compound concentrations tested in the present transcriptomic study were selected on the basis of our concentration-range finding studies on ESC differentiation inhibition. We selected a flusilazole concentration range that was equally spaced on a logarithmic scale and that covered the complete concentration-response range. Six concentrations of flusilazole $(0.54,1.8,5.4,18,27$ and $54 \mu \mathrm{M})$ were selected and we exposed ESC differentiation cultures from the embryoid body (EB)-stage at day three onwards. Cultures were microscopically evaluated at day 10 of the culture to assess the inhibition of cardiomyocyte differentiation, while parallel cultures were used for gene expression analysis at earlier time points as explained below. In each experiment, ESC differentiation inhibition was evaluated by calculating the mean scores of 24 exposed cultures per compound concentration relative score to the mean scores of 24 control cultures [214]. The ESC differentiation at the concentrations tested in the transcriptomic experiment and the 
concentration at which ESC differentiation is reduced by $50 \%\left(\mathrm{ID}_{50}\right)$ were calculated on the basis of the curve fit.

RNA isolation and whole genome gene expression profiling

RNA from flusilazole-exposed cultures was sampled 24h after the start of exposure (culture day 4, 8 replicates per group), and RNA from DMSO-exposed control differentiation cultures was sampled after 0, 24 and 48h from the EB-stage at day 3 (culture day 3, 4, and 5, 8 replicates per group). RNA isolation and preparation of the corresponding RNA samples for gene expression analysis using the Mouse Genome 4302.0 arrays (Affymetrix) was done as described previously [194]. Quality controls, including scaling factors, average intensities, present calls, background intensities, noise, and raw $Q$ values, were performed according to the manufacturer's instructions and showed to be within acceptable limits for all chips.

Data analysis and statistics

Normalization of Affymetrix CEL files was performed using the Robust Multichip Average (RMA) algorithm [179] with RMAexpress [180]. Probe to gene mapping was done as described previously [194]. Of the hybrid probe-set definitions included in the custom annotation, 16,331 probe sets defined by the Brainarray custom Chip Description File (CDF) version 11 (http://brainarray.mbni.med.umich.edu/Brainarray/Database/CustomCDF) [182] and 4,648 additional probe sets defined by Affymetrix chip annotation 26 were used in further analyses, giving a total of 20,979 probe sets. Probe sets for Affymetrix internal controls or probe sets that did not correspond to an Entrez Gene ID were not used in further analyses. Raw and normalized data are available at ArrayExpress (http:// www.ebi.ac.uk/arrayexpress/) under accession number E-TABM-1027.

Statistical analyses were carried out using the R statistical software environment version 2.9.1 (http://www.r-project.org) and the R-packages DNAMR and Limma using logtransformed values. For each gene, maximal fold ratios (FR) in gene expression between the experimental groups were determined by comparing the average normalized signal values per group and were calculated as the maximum/minimum ratio. Genes that were significantly differentially expressed between any of the non-exposed experimental groups were identified by a one-way ANOVA, using the cut-off criteria of a $p<0.001$ and $F R>1.5$. Six pairwise $t$-tests were performed to identify significantly transcriptionally responsive genes between each treatment group and its time-matched unexposed control group using the cut-off criterion of $p<0.001$.

To identify the biological processed altered by flusilazole-exposure, we studied functional annotation and enrichment for Gene Ontology (GO) biological processes using the tools on the DAVID website [183]. GO identifiers (GOID) were selected if the $p$-value was less than 0.001 in at least one of the experimental groups. Furthermore, the number 
of genes changed within a certain GOID had to be either greater than 30 or less than 300 to exclude GOIDs that are either too specific or too general. The absolute average fold change of the genes that caused enrichment in at least one of the experimental groups was calculated for a subset of the identified enriched GOIDs for each flusilazole-exposed experimental group. These subsets were generated based on the specificity of the GOID: child terms were selected above parent terms in cases where both parent and child terms were enriched [217]. For more detailed analyses of regulated GOIDs, we selected six GOID that reflected the flusilazole-induced response on the three identified themes, 'development', 'lipid metabolism' and 'cell division'. Within each theme, the most specific GOID was selected, i.e. comprising the lowest number of genes. Since the majority of the enriched GOIDs were related to development we selected four GOIDs that were related to this category, and one GOID that was related to the other two categories.

Definition of the differentiation track

To visualize the normal ESC differentiation, we defined the 'differentiation track' of these cultures as we described previously [177]. In short, PCA analysis was performed using the previously defined gene sets 'Van_Dartel_heartdiff_24h' [111] and 'EST biomarker genes' [194]. Thereafter, the chronological order of the control ESC differentiation groups in the PCA plot represented the 'differentiation track': a continuous representation of ESC differentiation based on the dynamics of gene expression. Coordinates along the first and second principal component were calculated for each sample. Deviation of flusilazole-exposed cultures from the time-matched controls on the 'differentiation track' was analyzed by applying a Hotelling $t$-test to these coordinates. Significant deviation from the 'differentiation track' $(p<0.05)$ was considered characteristic for developmental toxicants. In addition, we created heatmaps of the genes corresponding to these earlier defined gene sets 'Van_Dartel_heartdiff_24h' and 'EST biomarker genes' to visualize gene expression using GeneMaths XT (Applied Maths, Sint-Martens-Latem, Belgium).

Validation gene expression using real-time $P C R$

Transcriptomic results were validated using real-time PCR (RT-PCR). RNA of all samples per experimental group was pooled and used to generate CDNA by using the high-capacity CDNA archive kit containing random hexamer primers (Applied Biosystems, Foster City, CA). mRNA presence was measured in duplo with Taqman gene expression assays (Applied Biosystems) on a 7500 Fast Real-Time PCR System, with a two-step PCR procedure according to the manufacturer's protocol. The thermal cycling conditions were $95^{\circ} \mathrm{C}$ for $20 \mathrm{~s}$ for the first cycle, followed by 40 cycles of $95^{\circ} \mathrm{C}$ for $3 \mathrm{~s}$ and $60^{\circ} \mathrm{C}$ for $30 \mathrm{~s}$. The mRNA markers we used were Hoxa1 (Mm00439359_m1) and Mesp2 (Mm00655937_m1) both annotated to anterior posterior pattern formation, and Cyp51 (Mm00490968_m1) and Sc4mol (Mm00499386_m1) both annotated to sterol biosynthesis. Tbp was selected 
as housekeeping gene for Hoxa1 and Sc4mol, Polr2a was selected as housekeeping gene for Mesp2 and Cyp51. The mRNA expression was normalized to the value of the housekeeping gene for each reaction according to the comparative $\mathrm{Ct}$ method and expressed relative to the expression level in unexposed cultures. Statistical gene expression regulation was calculated by performing a Student's t-test. Results were considered statistically significant if $p<0.05$.

\section{Results}

Flusilazole-induced effects on cell viability and differentiation

Flusilazole caused a concentration-dependent decrease in cell viability, with $<80 \%$ cell viability at the two highest flusilazole concentrations that were tested in the transcriptomic study (Figure 1 and Table 1). The calculated ID $D_{50}$ and $I C_{50}$ of flusilazole were 5.9 and $55.9 \mu \mathrm{M}$ respectively, showing that ESC differentiation is a more sensitive end point as compared to cell viability. Except for the lowest concentration tested, all tested flusilazole concentrations affected ESC differentiation in a concentration-dependent manner, up to $100 \%$ inhibition at the highest test concentration tested (Figure 1 and Table 1).

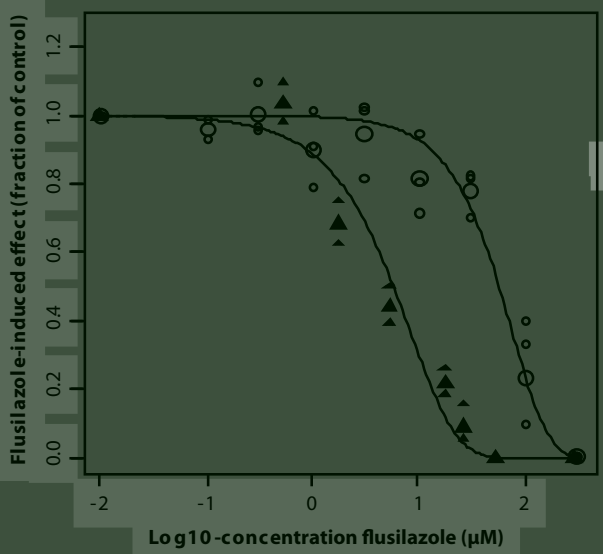

Figure 1. Concentration-response curves of ESC differentiation (triangles; $n=2$ ) and cell viability (circles; $\mathrm{n}=3$ ) after flusilazole exposure. Large symbols represent the mean of replicate measurements. The curves are fitted using the log-logistic model, showing the solvent control samples on the left of the curve.

Flusilazole-induced gene expression changes

Gene expression was compared between flusilazole-exposed ESC differentiation cultures and time-matched solvent control-exposed cultures to identify significantly differentially expressed genes (Table 1$)$. At the lowest test concentration $(0.54 \mu \mathrm{M})$, 
Table 1. Number of significantly differentially expressed genes $(p<0.001)$ after $24 \mathrm{~h}$ exposure to a concentration range of flusilazole. ESC differentiation (exposure days $0-10$ ) and cell viability (exposure days $0-3$ ) at the concentrations tested in this transcriptomic study were calculated using the curve fit of the data presented in Figure 1.

\begin{tabular}{ccccc}
\hline $\begin{array}{c}\text { Concentration } \\
\text { of flusilazole } \\
\text { exposure }\end{array}$ & $\begin{array}{c}\text { ESC } \\
\text { differentiation } \\
\text { (percent of } \\
\text { control) }\end{array}$ & $\begin{array}{c}\text { Viability } \\
\text { (percent of } \\
\text { control) }\end{array}$ & $\begin{array}{c}\text { Common genes } \\
\text { Differentially } \\
\text { expressed genes after } \\
\text { flusilazole exposure* } \\
\text { (n) }\end{array}$ & $\begin{array}{c}\text { differentially expressed } \\
\text { both during ESC } \\
\text { differentiation** } \\
\text { and after flusilazole } \\
\text { exposure* } \\
\text { (n (\%)) }\end{array}$ \\
\hline $0.54 \mu \mathrm{M}$ & $93.9 \%$ & $94.9 \%$ & 108 & $27(25.0 \%)$ \\
$1.8 \mu \mathrm{M}$ & $80.9 \%$ & $94.6 \%$ & 372 & $118(31.7 \%)$ \\
$5.4 \mu \mathrm{M}$ & $53.0 \%$ & $93.2 \%$ & 377 & $185(49.1 \%)$ \\
$18 \mu \mathrm{M}$ & $12.1 \%$ & $84.8 \%$ & 552 & $258(46.7 \%)$ \\
$27 \mu \mathrm{M}$ & $4.2 \%$ & $77.4 \%$ & 1706 & $871(51.1 \%)$ \\
$54 \mu \mathrm{M}$ & $0.2 \%$ & $53.9 \%$ & 2010 & $870(43.3 \%)$ \\
\hline
\end{tabular}

* Genes differentially expressed in exposed versus unexposed $24 \mathrm{~h}$ experimental groups ( $p<0.001$ and FR $>1)$

** Genes differentially expressed among $0 \mathrm{~h}, 24 \mathrm{~h}$ and $48 \mathrm{~h}$ unexposed experimental groups $p<0.001$ and FR $>1.5$ ( $n=3780$ genes)

where no inhibition of ESC differentiation or cell viability was observed, 108 genes were significantly differentially expressed. The number of differentially regulated genes appeared to increase in a monotonic fashion with increasing concentration of flusilazole exposure, with a total number of 2010 genes significantly differentially expressed at the highest test concentration $(54 \mu \mathrm{M})$.

We identified 3780 genes $(p<0.001$ and fold ratio $(F R)>1.5)$ that were regulated with time in the unexposed experimental groups. An increasing percentage of flusilazoleregulated genes overlapped with these ESC differentiation-related genes, reaching a plateau of approximately $50 \%$ of genes commonly expressed at $5.4 \mu \mathrm{M}$ flusilazoleexposed cultures (Table 1). These high percentages of genes that overlap with the genes identified in ESC differentiation indicate a specific differentiation-modulating response. Exposure to $54 \mu \mathrm{M}$ flusilazole resulted in a similar number of genes that were shared with the ESC differentiation-regulated genes $(n=871)$ as compared to the $27 \mu \mathrm{M}$ exposure group ( $n=870)$, showing no additional differentiation modulation at the highest test concentration.

To study the effects of flusilazole exposure at the level of biological processes, we studied GO enrichment of the regulated genes. These analyses revealed a total of 94 enriched GOIDs in at least one of the flusilazole-exposed experimental groups (Supplementary Table 1). The majority of genes modulated by flusilazole exposure function in developmental-related processes (66 GOIDs). In addition, over-represented GOIDs were related to other processes such as 'lipid metabolism' (9) and 'cell division' (9). The fold 
A

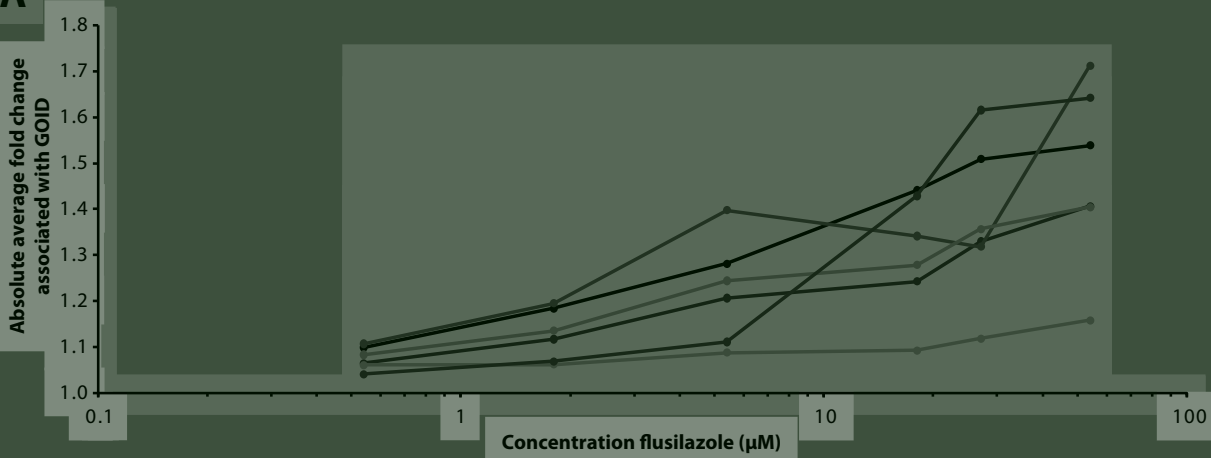

B

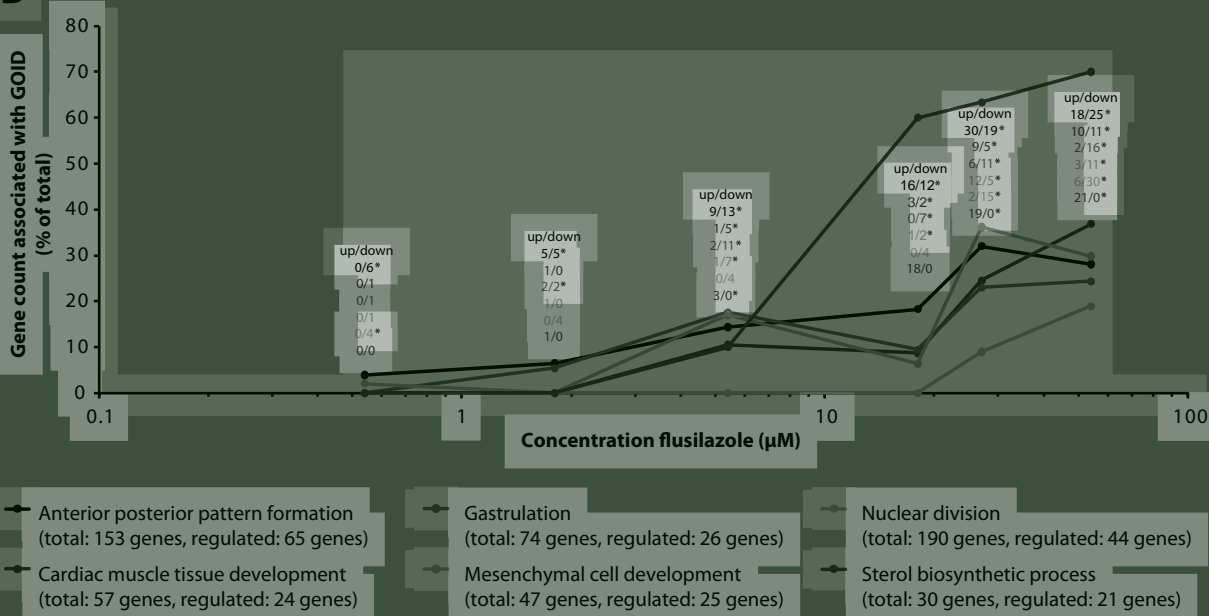

Figure 2. (A) Concentration-related effect of flusilazole exposure in ESC differentiation cultures on absolute average fold change of a subset of enriched GOID. For comparability reasons the absolute average fold change was calculated using the significantly differentially expressed genes that contributed to the enrichment in at least one of the experimental groups. (B) Concentration-related effect of flusilazole exposure in ESC differentiation cultures on relative gene count associated with Gene Ontology Identifiers (GOID). GOID in red-yellow are related to development, in green to cell division and blue to lipid metabolism. 'Total' refers to total number of genes annotated to that GOID, and 'regulated' refers to total number of statistically significant genes that contributed to the enrichment of that GOID in at least one experimental group. Asterisks indicate statistically significantly enriched GOID. For color figure, see Color figures section.

change inductions show clear concentration-related effects at the level of GOIDs (Figure $2 \mathrm{~A}$ ) as well as at the individual gene level as shown for a subset of enriched GOIDs (Figure 3). Furthermore, the enrichment of the GOIDs also appeared to be concentration-related as observed by a higher relative gene count with increasing test concentrations (Figure 2B).

GO analyses revealed three major themes of related GOIDs impacted by flusilazole: development, lipid metabolism, and cell division. Out of these three categories of genes, 
A

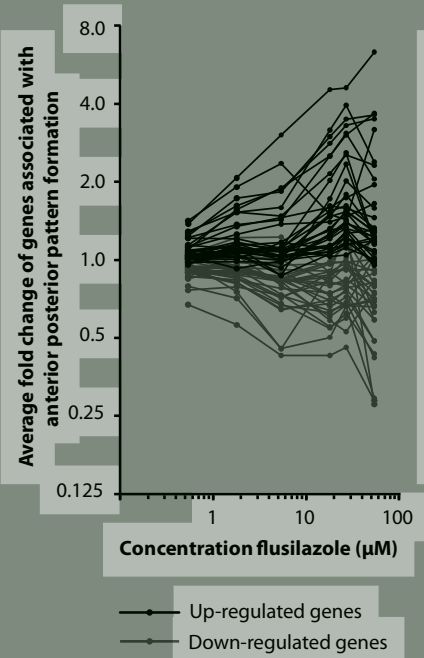

B

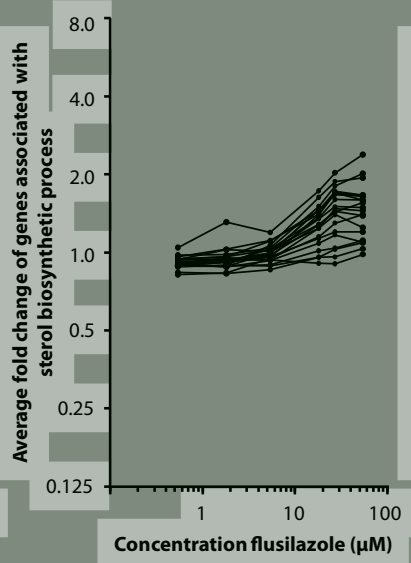

C

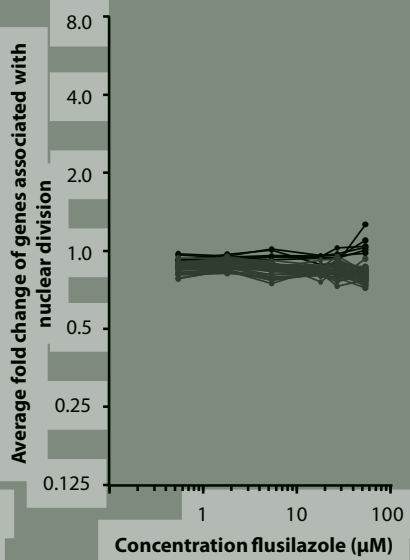

Figure 3. Average fold change of individual genes that were significantly differentially expressed in at least one experimental group and contributed to the significant enrichment of 'anterior posterior pattern formation' (A), 'sterol biosynthetic process' (B) and 'nuclear division' (C).

GOIDs related to development appeared to be most sensitive to flusilazole exposure, showing over-representation already after exposure to $5.4 \mu \mathrm{M}$. Genes that contributed to the enrichment of the development-related GOIDs included both up- and downregulated genes (Figure $2 \mathrm{~A}$ and $3 \mathrm{~A}$ ). The most significant enrichment and highest relative gene count was obtained for lipid metabolism-related GOIDs (Supplementary table 1 and Figure 2A). The lowest concentration of flusilazole at which this enrichment was observed was $18 \mu \mathrm{M}$. The genes that were responsible for enrichment of the lipid metabolism-related GOIDs were all up-regulated after flusilazole exposure (Figure 2A and $3 \mathrm{~B}$ ). The significantly enriched GOIDs related to cell division showed pronounced over-representation at the two highest test concentrations only as reflected by the relative gene count (Figure $2 \mathrm{~A}$ ). Figure $3 \mathrm{C}$ shows the differentially expressed genes that contributed to the enrichment of the GOID 'nuclear division'. Most of the genes positively related to cell cycling were down-regulated by flusilazole exposure, whereas the majority of cell cycle genes up-regulated by flusilazole treatment appeared involved in cell cycle arrest (not shown).

RT-PCR analyses revealed that Hoxa1 and Mesp2, annotated to the developmentalrelated process 'anterior posterior pattern formation', were significantly differentially expressed by flusilazole exposure from respectively 0.54 and $1.8 \mu \mathrm{M}$ onwards (Supplemental Figure 1). In addition, the significant up-regulation of the expression level of both Cyp51 and Sc4mol, involved in sterol biosynthesis, was shown by RT-PCR from 

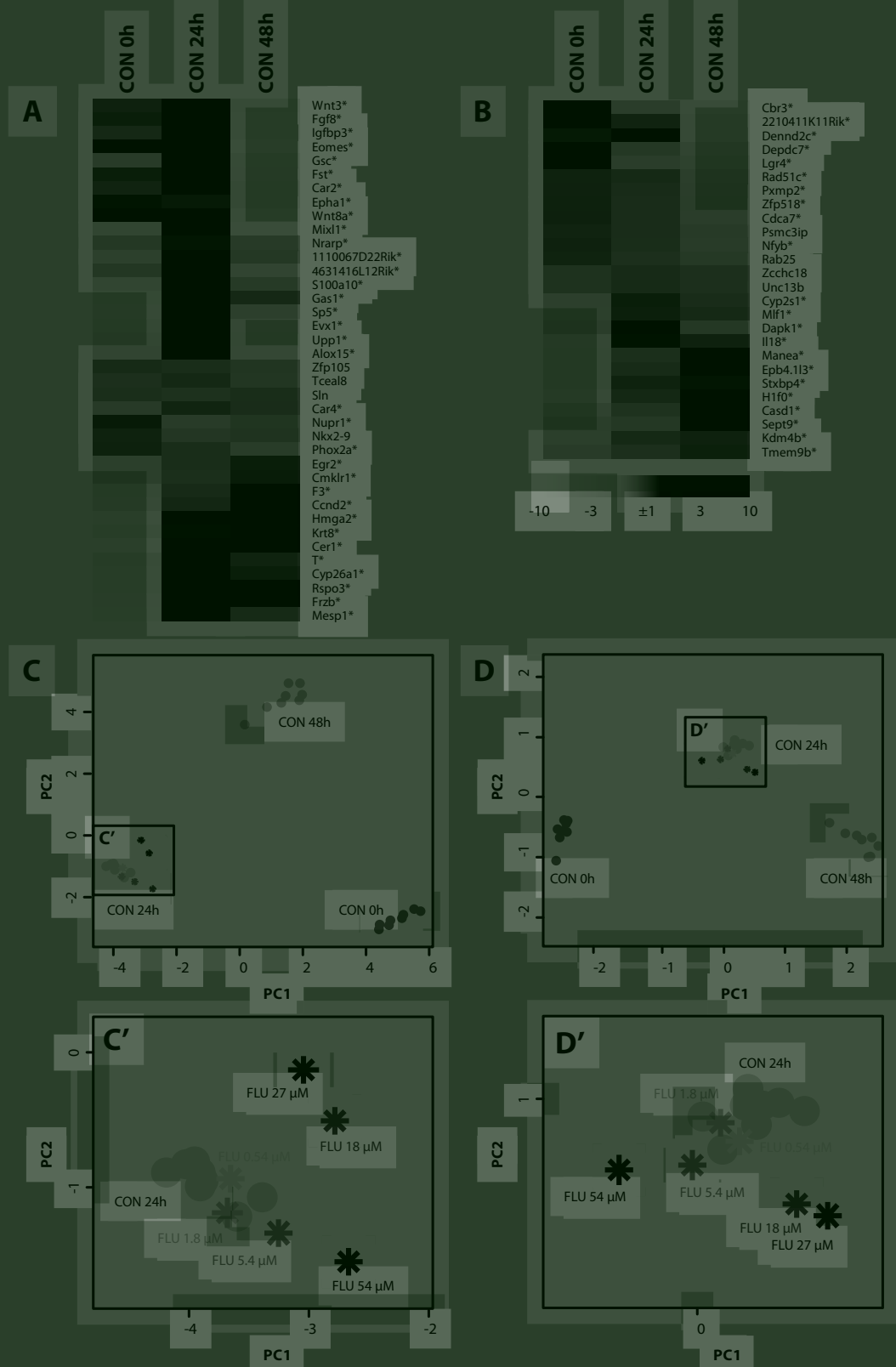

Figure 4. Mean gene expression of the 'Van_Dartel_heartdiff_24h' (A) and 'EST biomarker genes' (B) gene sets at $0 \mathrm{~h}, 24 \mathrm{~h}$, and $48 \mathrm{~h}$ of control ESC differentiation cultures (CON). ${ }^{*}$ Indicate significantly differentially expressed genes among the $0 \mathrm{~h}, 24 \mathrm{~h}$, and $48 \mathrm{~h}$ CON groups. Principal Component Analysis of the control ESC differentiation cultures (individual samples, circles) at $0 \mathrm{~h}, 24 \mathrm{~h}$, and $48 \mathrm{~h}$ of ESC differentiation and flusilazole-exposed cultures (mean of $n=8$ samples, asterisks) using the 'Van_Dartel_heartdiff_24h' (C) and 'EST biomarker genes' (D). C' and D' present the enlargement of the CON $24 \mathrm{~h}$ samples and the flusilazoleexposed cultures (FLU). For color figure, see Color figures section. 
respectively 5.4 and $18 \mu \mathrm{M}$ onwards (Supplemental Figure 1). All evaluated genes follow a clear concentration-dependent gene expression regulation.

Concentration-related deviation of flusilazole-exposed cultures from differentiation track

The modulation of ESC differentiation was further studied by analyzing the concentration-related deviation from the 'differentiation track' defined by PCA. The gene sets used for defining the 'differentiation track' were defined previously, and represent slightly different stages of ESC differentiation. The 'Van_Dartel_Heartdiff_24h' genes were all up-regulated at $24 \mathrm{~h}$ as compared to earlier time points, while the 'EST biomarker genes' gene set includes genes that were up- or down-regulated in ESC differentiation cultures sampled up to $48 \mathrm{~h}$ of differentiation. Gene expression showed that the majority of the genes within these sets were also differentially expressed among ESC differentiation cultures sampled after 0, 24 and $48 \mathrm{~h}$ in this study, namely $84 \%$ and $85 \%$ in the 'Van_Dartel_Heartdiff_24h' and 'EST biomarker genes' gene set respectively (Figure 4A and B).

Table 2. Significance of deviation of flusilazole-exposed cultures from the time-matched controls on the defined 'differentiation track' ( $p$-value) using selected gene sets.

\begin{tabular}{ccc}
\hline $\begin{array}{c}\text { Concentration of flusilazole } \\
\text { exposure }\end{array}$ & $\begin{array}{c}\text { 'Van_Dartel_Heartdiff_24h'a } \\
\text { genes) }\end{array}$ & $\begin{array}{c}\text { 'EST Biomarker genes } \\
\mathbf{s}^{\mathbf{b}}\end{array}$ \\
\hline $0.54 \mu \mathrm{M}$ & 0.346 & 0.298 \\
$1.8 \mu \mathrm{M}$ & 0.077 & 0.370 \\
$5.4 \mu \mathrm{M}$ & $2.5 \mathrm{E}-03^{*}$ & $3.7 \mathrm{E}-02^{*}$ \\
$18 \mu \mathrm{M}$ & $1.1 \mathrm{E}-02^{*}$ & $4.8 \mathrm{E}-04^{*}$ \\
$27 \mu \mathrm{M}$ & $3.0 \mathrm{E}-06^{*}$ & $5.5 \mathrm{E}-07^{*}$ \\
$54 \mu \mathrm{M}$ & $6.6 \mathrm{E}-07^{*}$ & $1.5 \mathrm{E}-04^{*}$ \\
\hline
\end{tabular}

* Significant $p$-value for deviation from differentiation track

a van Dartel et al. (2009a)

${ }^{\mathrm{b}}$ van Dartel et al. (2010b)

Table 2 presents the $p$-values for significance of deviation from the 'differentiation track' for flusilazole-exposed cultures. The coordinates of ESC differentiation cultures exposed to 0.54 and $1.8 \mu \mathrm{M}$ flusilazole versus time-matched unexposed ESC differentiation were not statistically significantly different in both PCA figures representing the differentiation tracks (Table 2, Figure $4 \mathrm{C}$ and D). At concentrations of $5.4 \mu \mathrm{M}$ flusilazole and higher, ESC differentiation cultures appeared to deviate significantly from both differentiation tracks. In both PCA plots, the position of flusilazole-exposed cultures deviated concentration-dependently away from their time-matched unexposed cultures (CON 24 h) and towards the CON Oh samples at the lower three concentrations, to subsequently turn towards the CON 48h samples at the next higher two concentrations. The position of the highest test concentration in the PCA fell outside this smooth deviation pattern. 


\section{Discussion}

In recent years, toxicogenomic has proven a valuable tool in predictive toxicology [37, 41]. Early initiatives to use in vitro alternatives combined with transcriptomics for the prediction of toxicity have shown promising results $[45,218]$. Several studies have addressed molecular end points in the assessment of developmental toxicity in various models [25, 30, 49, 219-221]. We hypothesized that combining the EST with toxicogenomics may lead to an improved model for in vitro developmental toxicity prediction by optimization of important aspects, including culture duration, end point evaluation, and definition of the predictability and applicability domain. In a previous paper [214], we presented evidence that the detection of developmental toxicity by transcriptomics in EST was influenced by the compound concentration tested. In the present study, we investigated the concentration-dependent transcriptomic responses to flusilazole in ESC differentiation cultures and their impact on the detection of developmental toxicity.

Concentration-dependent flusilazole-induced effects on ESC differentiation cultures

Flusilazole appeared to affect both ESC differentiation and cell viability, with effects on ESC differentiation occurring at roughly 10-fold lower concentrations indicating specific flusilazole-induced differentiation-modulating effects. In addition, gene expression analysis revealed that approximately $50 \%$ of the genes regulated after flusilazole exposure also appeared to be regulated during ESC differentiation. This finding is in line with our previous study in which gene expression signatures of differentiation modulators showed a significant overlap with ESC differentiation-related genes [194]. In the present study, the effect of flusilazole on ESC differentiation was observed by transcriptomics at the lowest concentration tested, with developmental-related genes such as $\operatorname{Tdfg} 1$, Mesp2 and Otx1 [222-224] significantly differentially expressed (not shown).

Analyses based on enrichment of GOIDs were used to interpret the significant gene expression changes in ESC differentiation cultures exposed to flusilazole and to provide additional information about concentration-dependent ESC differentiation modulation. Most of the enriched GO terms included developmental processes, the remaining enriched GOIDs were cell division- and lipid metabolism-related processes and leaving only a few terms unassigned. Increasing flusilazole concentrations resulted in a higher gene count of GOIDs, and in addition the fold change of these genes increased with the test concentration, an effect that has also been described previously in other models $[189,225]$. Enrichment of developmental-related GOIDs increased concentrationdependently and was already observed at the lowest tested concentration, indicating that developmental processes were the most sensitive process affected, which was confirmed by RT-PCR. Cell division-related processes were mainly enriched at the two 
highest test concentrations, which anchors the phenotypically observed cytotoxicity of flusilazole.

Besides the flusilazole-induced effects on ESC differentiation and cell viability, flusilazole exposure also resulted in a clear concentration-dependent perturbation of the sterol biosynthesis pathway, and other lipid metabolism-related processes, as observed by DAVID analyses. This relates to the specific antifungal mode of action of triazoles, including flusilazole, which is inhibition of lanosterol-14a-demethylase (Cyp51), an enzyme required for sterol biosynthesis in all eukaryotic systems [226, 227]. The affinity of the mammalian Cyp51 to flusilazole is 100 times lower than that of its fungal counterpart, which explains its effective use as fungicide [228]. RT-PCR results confirmed the transcriptomic results by showing a clear concentration-dependent induction of Cyp51 expression that was statistically significant from $5.4 \mu \mathrm{M}$ onwards. The gene expression response caused by triazoles in rats revealed the differential expression of genes involved in lipid metabolism, including sterol biosynthesis [51, 229]. Five of the 10 genes targeted by the triazole triadimefon within the sterol biosynthesis pathway [51] were also affected in our ESC differentiation model after flusilazole exposure. This finding demonstrates that the compound's mode of action was also detected using transcriptomics in the EST. This perturbed lipid metabolism could have played a role in the decreased cell viability observed at the highest test concentrations, as is also observed in fungi after exposure to triazoles [228].

Although studying gene expression response of complete GOID is very useful, the gene expression profile of individual genes within GOID may provide additional information about the induced gene expression response [230]. Within the present study, we observed that the genes in the enriched functional classes related to development were all concentration dependently regulated, although some were up-, and others or downregulated with diverse magnitudes among the genes studied, which we showed for a subset of GOID in Figure 2A and 3A. This finding is reminiscent of the complexity of gene expression alterations which accompany embryonic cell differentiation. On the other hand, we observed consistent concentration-dependent up-regulated gene expression profiles for the lipid metabolism-related GO terms, reminiscent of the mode of action of triazoles (see above). Conversely, we observed cell division-related GO terms were essentially down-regulated concentration-dependently. Within the GO term 'nuclear division', only six genes showed an opposite expression profile as they were up-regulated by flusilazole treatment and these appeared involved in cell cycle arrest, while most of the genes that were down-regulated by flusilazole exposure function in cell cycling. These observations matched the cytotoxic response of flusilazole at 27 and $54 \mu \mathrm{M}$. 
Detection of developmental toxicity using the differentiation track

Flusilazole-induced differentiation modulation was further studied using the'differentiation track' approach. This approach takes advantage of the gene expression dynamics of differentiating ESC [177, 231], or embryonic development [232] which can be reflected in a PCA-based differentiation track. We have shown previously that developmentally toxic compounds could be identified by their deviation from the 'differentiation track' [177]. We used the 'Van_Dartel_heartdiff_24h' and the 'EST biomarker genes' gene sets to define differentiation tracks, which both clearly reflected the dynamics of ESC differentiation as shown in the PCA figures. A vast majority of the genes within these gene sets were significantly regulated among the control experimental groups in the present study. Moreover, the pattern of regulation highly reflected the initial gene expression profile. The 'Van_Dartel_heartdiff_24h' gene set includes genes that were up-regulated at $24 \mathrm{~h}$, whereas the 'EST biomarker genes' were identified by their dynamic gene expression during the first $48 \mathrm{~h}$ of ESC differentiation. These differences in gene expression in time were reflected in the different projection of the experimental samples in both PCA plots. Nevertheless, the mutual distances among experimental samples in both PCA plots were comparable, demonstrating the robustness of the approach.

The use of this 'differentiation track' approach for the identification of the developmental toxicity of compounds relies on their effect on the expression signature of the entire gene set ('Van_Dartel_heartdiff_24h' or 'EST_biomarker genes'). We and others have shown previously that individual genes within a process need not necessarily be significantly regulated by exposure, while on the level of gene sets compound-induced effects can still be captured effectively $[111,233]$. Within the present study we showed that the 'differentiation track' approach is useful for the identification of concentrationrelated ESC differentiation modulation. The concentration-related increase in significance of differentiationtrack deviation observed in flusilazole-exposed cultures was in agreement with our morphological scoring and $\mathrm{GO}$ analysis results.

Furthermore, besides the use of the significance of 'differentiation track' deviation, the direction of 'differentiation track' deviation of compound-exposed cultures may give additional information on the nature of effects on ESC differentiation cultures. The projections of the lower three flusilazole concentrations $(0.54,1.8,5.4 \mu \mathrm{M})$ appeared in almost the same directions relative to controls. However, the projections of the subsequent two flusilazole concentrations ( 18 and $27 \mu \mathrm{M})$ deviated from this pattern towards the CON 48h samples. This may be due to the pronounced induction of sterol biosynthesisrelated genes starting at $18 \mu \mathrm{M}$ (Figure 2). The PCA projection of cultures exposed to the highest concentration of flusilazole $(54 \mu \mathrm{M})$ discontinued again from the pattern of lower concentrations. This may be explained by the cytotoxic effects as evidenced both by gene expression (Figure 2) and morphological end points (Figure 1) that occurred uniquely at the concentration of $54 \mu \mathrm{M}$ flusilazole in ESC differentiation cultures. 
In the present study, compound concentrations were selected on the basis of the morphological outcome of the 'classical' EST, an approach that has been used frequently $[190,191]$. When testing a compound with unknown toxicity, test concentration selection could be based on cytotoxicity data. Our results suggest the need to include compound concentrations below the range of cytotoxicity, since this is important for acquiring information about the relative sensitivity of ESC differentiation modulation as compared to cytotoxicity. Additional studies testing (non-)developmental toxicants with different potencies are needed to evaluate the usefulness of this approach of concentration selection.

Conclusions

We showed previously that the degree of compound-induced ESC differentiation inhibition correlated to the significance of deviation from the 'differentiation track' [214]. In the present study, we show concentration-dependent effects on multiple cellular processes in flusilazoleexposed cultures, and identified the relative sensitivity of enriched processes. We observed a higher sensitivity of developmental-related processes as compared to cell division-related processes, and in addition confirmed the sterol synthesis pathway mediated mode of action of flusilazole toxicity by differential gene expression. Furthermore, gene expression regulation of the gene sets 'Van_Dartel_heartdiff_24h' and 'EST biomarker genes' using the 'differentiation track' approach illustrated that the detection of the differentiation modulating properties of flusilazole in EST is concentration-dependent. In conclusion, studying gene expression modulation by compounds within the EST protocol allows a more detailed description of the effects of the test compound at the molecular level as compared to the 'classical' EST. In addition, the choice of test concentrations proved an essential aspect of study design in view of the predictability of the assay for the detection of developmental toxicants.

\section{Acknowledgements}

The authors acknowledge the useful discussions on this work with Joshua F. Robinson.

This work was supported by the Dutch technology society foundation STW [grant MFA6809]. The sponsor was not involved in the design and conduct of the study; collection, management, analysis, and interpretation of the data; and preparation, review, or approval of the manuscript.

\section{Supplementary data}

Supplementary data associated with this article can be found at doi:10.1016/j. taap.2010.12.008. 
Dorien A.M. van Dartel, Jeroen L.A. Pennings, Joshua F. Robinson, Jos C.S. Kleinjans, Aldert H. Piersma

Toxicology Letters (2011) 201:143-151 
CHAPTER 8

Discriminating classes of developmental toxicants using gene expression profiling in the Embryonic Stem cell Test 


\section{Abstract}

The Embryonic Stem cell Test (EST) has been shown to be a promising in vitro method for the prediction of developmental toxicity. In our previous studies, we demonstrated that the implementation of gene expression analysis in the EST may further improve the identification of developmental toxicants. In the present study, we investigated if gene expression profiling could be used to discriminate compound classes with distinct modes of action (MoA) using the EST protocol. Gene expression data of our previous study were used and were analyzed of embryonic stem cell (ESC) differentiation cultures exposed to six compounds belonging to two classes with distinct MoA, namely phthalates and triazoles. We used three approaches to study class-characteristic gene regulation that may be useful for discrimination of compound classes. First, at the individual gene level, gene signatures characteristic for each class were identified that successfully discriminated both classes using Principal Component Analysis. Second, at the functional level, enriched gene ontology (GO) biological processes showed their usefulness for class discrimination via hierarchical clustering. Third, two previously identified gene sets, which we designed to predict developmental toxicity, appeared successful in separating phthalate from triazole compounds. In summary, we established the possibility to discriminate between compound classes in the EST system using three different specific transcriptomics-based approaches. Differential gene expression information specific for the class of compound under study may be employed to optimize prioritization of compounds within that class for further testing. 


\section{Introduction}

According to international legislation, current toxicity testing is primarily assessed using laboratory animals. In vivo studies are expensive, requiring a considerable number of experimental animals, and raise important ethical concerns. For these reasons, European policy is promoting alternative testing methods and assessment strategies to reduce the use of laboratory animals and whenever possible, replace animals employed for toxicological studies [234]. Although none of the existing in vitro methods that have been identified is capable of replacing the existing classical in vivo approach, a select few have been recognized as potential alternative methods for the assessment of developmental toxicity [20].

One of the most studied in vitro alternatives for developmental toxicicity screening is the murine Embryonic Stem cell Test (EST). Our transcriptomic studies suggest that the implementation of toxicogenomics-based assessments into the EST may lead to improved prediction, by providing detailed information regarding modulation of embryonic stem cell (ESC) differentiation in relation to concentration and time [177, 214, 235] and furthermore, enabling the prediction of toxicity based on pre-determined gene lists related to ESC differentiation [214].

Diverse studies have shown that discrimination of compounds with similar modes of action is possible using biological activity profiling patterns. Compound discrimination has been used predominantly in drug discovery [236, 237], and this application has also been studied to assess its potential for toxicological screening [238, 239]. For example, Martin et al. demonstrated successful grouping of compounds with similar toxicological effects using signatures characteristic for specific toxic outcome (i.e. hepatomegaly and PXR activation). Application of compound discrimination analyses is of interest for ranking of structurally related compounds with unknown toxicity for further testing. Many new compounds or compounds with insufficient toxicological data are structurally related to compounds of which a wealth of toxicological data is available. Before deciding on generating a full toxicological profile of the new compound, it may be useful to compare the gene expression profile in an appropriate in vitro test of the new with the well-studied compound(s). If a comparable gene expression pattern is observed it may be possible to rely on the data of the tested compound as a surrogate for assessing the toxic properties of the new compound [240]. This approach has been supported by studies that were able to distinguish [241] or rank [242] similarly-structured compounds for their toxic potencies using gene expression profiling. Furthermore, discrimination of compounds based on specific signatures is of interest for prioritizing structurally-related compounds with unknown toxicity for further testing.

The objective of the present study was to evaluate the ability of the EST to categorize compounds with a similar mode of action by applying a toxicogenomic approach. In our 
previous study, we evaluated the identification of developmental toxicants using gene expression analysis [194]. In this study, using this dataset, we assessed common and differential gene expression regulation patterns of triazole and phthalate compounds, and evaluated whether these two classes are discriminated based on their gene expression response in the EST system. We selected these two classes of compounds on the basis of the availability of effect data in the EST, suggested different mechanisms of toxicity, and furthermore, the relevance of their effects on human health.

Triazoles are often used in agricultural settings as well as in pharmaceutical applications for their known antifungal effects. The fungicidal mode of action of triazoles is based on the inhibition of the enzyme lanosterol-14a-demetylase (Cyp51), which plays an essential role in sterol metabolism and is required for the synthesis and integrity of the fungal cell wall [227]. In addition, triazoles may affect also other Cyp-enzymes, which may result in developmental malformations when embryos are exposed prenatally [243, 244]. The observed effects include urogenital and skeletal malformations [201]. The triazole compounds that were selected for the present study were flusilazole (FLU), hexaconazole (HEX) and triadimefon (TDI) which all contain the 1,2,4-triazole moiety (Figure 1).

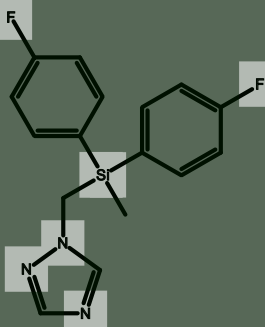

Flusilazole<smiles>COC(=O)c1ccccc1C(=O)O</smiles>

Monomethyl phthalate

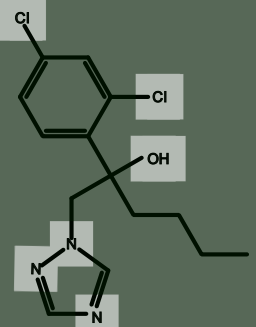

Hexaconazole

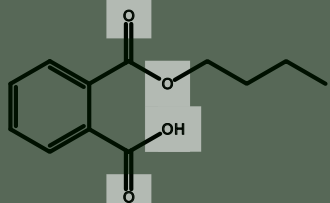

Monobutyl phthalate

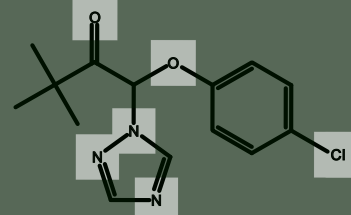

Triadimefon

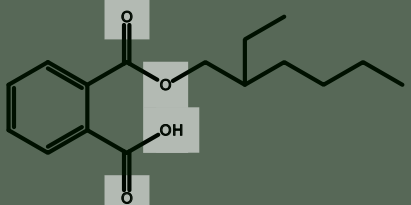

Monoethylhexyl phthalate

Figure 1. Chemical structures of triazoles and phthalates tested in the present study.

The second class of compounds included in the present study are phthalates, which are esters of phthalic acid (benzene-1,2-dicarboxylic acid) in ortho configuration. These compounds are widely used as plasticizers to increase the flexibility of a variety of PVC products like toys, vinyl floors and catheters. Developmental toxicity studies have shown 
that the monoester phthalates containing side-chain lengths of C4-C6 may induce teratogenic effects [121]. In the present study, we included two developmentally toxic phthalates, monobutyl phthalate (MBP; C4), and monoethylhexyl phthalate (MEHP; C6), as well as one non-developmentally toxic phthalate monomethyl phthalate (MMP; 1 ). Several modes of action have been suggested for the teratogenic properties of phthalates, including intracellular acidification, altered cellular proliferation and induction of DNA damage $[245,246]$. Malformations induced by the developmental toxic phthalates tested in the present study include external and skeletal malformations, cleft palate and hydrocephaly $[92,122,199]$.

In the present study, we evaluated the discriminatory ability of gene expression to classify triazoles and phthalates using multiple approaches based on 1) gene lists characteristic for each class identified in the present study 2) enriched Gene Ontology (GO) processes, and 3) gene lists derived from our earlier toxicogenomic studies that identify developmental toxicants in the EST. We could successfully segregate the tested compounds into two distinct classes using these approaches. It can be concluded that, as a proof-of-principle, our findings show the possibility of using gene expression data obtained from ESC differentiation inhibition for the discrimination of compound classes with different modes of action of developmental toxicity.

\section{Materials and methods}

Data of our previous study [214] were used for the analyses described here. The methods and data regarding culture conditions, compound exposures, assessment of differentiation inhibition, and gene expression profiling have been described in full detail [214]. The data have been deposited in EBI's ArrayExpress (http://www.ebi.ac.uk/arrayexpress) and are accessible through ArrayExpress accession number E-MTAB-300.

\section{Test compounds}

Previous studies showed that evaluating compounds at a concentration affecting differentiation by $50 \%\left(\mathrm{ID}_{50}\right)$ resulted in successful identification of differentiation modulating, without inducing general toxic responses $[111,177,194] . D_{50}$ concentrations were calculated using historical concentration-response data from our lab.

ESC differentiation cultures were exposed to $1.4 \mathrm{mM}$ monobutyl phthalate (MBP; CAS No. 131-70-4, TCI Europe, Zwijndrecht, Belgium), $1.4 \mathrm{mM}$ monomethyl phthalate (MMP; Cas No. 4376-18-5, TCI Europe), 0.68 mM monoethylhexyl phthalate (MEHP; CAS No. 4376-20-9, TCI Europe), 5.4 MM flusilazole (FLU; Cas No. 85509-19-9, Sigma-Aldrich, Zwijndrecht, The Netherlands), $21 \mu \mathrm{M}$ hexaconazole (HEX; CAS No. 79983-71-4, SigmaAldrich), $160 \mu \mathrm{M}$ triadimefon (TDI; CAS No. 43121-43-3, Sigma-Aldrich), or to solvent only 
(CON; $0.2 \%$ dimethyl sulfoxide (DMSO)). Compound concentrations used in this study did not result in cytotoxic effects and inhibited ESC differentiation effectively, except for MMP- and MBP-exposed cultures that showed no inhibition of ESC differentiation [214].

Derivation of class characteristic gene signatures

Statistical analyses were carried out using the R statistical software environment (http:// www.r-project.org) using log-transformed values. Maximal fold ratios (FR) in gene expression were determined for each gene by comparing the normalized signal of the compound-exposed samples relative to the average normalized signal of the DMSOexposed samples. Significant gene expression responses were identified by performing pairwise $t$-tests between each treatment group and the unexposed control group using a $p$-value cut-off of $<0.001$. The selected $p$-value corresponded to a false discovery rate of 0.03 over all compound comparisons in this study. The phthalate and triazole signatures were composed of the genes that were commonly differentially expressed with similar regulation by respectively the developmentally toxic phthalates MBP and MEHP, and by the three triazoles FLU, HEX and TDI.

The gene expression profile of the phthalate and triazole signature was visualized in a heatmap using GeneMaths XT (Applied Maths, Sint-Martens-Latem, Belgium). DAVID bioinformatics resources [183] was used to identify enrichment for Gene Ontology (GO) biological processes within the phthalate and triazole signatures. Functional annotation of genes was provided for parent terms that covered the identified enriched GO terms. In addition, we evaluated if genes within these signatures were among the previously identified genes that were regulated in early ESC differentiation [194].

Class discrimination - class signatures

The identified triazoles and phthalate signatures were used to study the discrimination of these compound classes, using Principal Component Analyses (PCA) with GeneMaths XT (Applied Maths, Sint-Martens-Latem, Belgium). In addition, control analyses were performed to study the robustness of our approach. First, we included available gene expression data of six other compounds (carbamazepine $(160 \mu \mathrm{M})$, methotrexate $(240$ $\mathrm{nM})$, methylmercury $(0.48 \mu \mathrm{M})$, nitrofen $(100 \mu \mathrm{M})$, saccharine $(5 \mathrm{mM})$, and warfarin (1.1 $\mathrm{mM})$ ) tested in ESC differentiation cultures according to a similar incubation protocol [214] to study the discrimination of phthalates or triazoles from a more diverse set of compounds, using respectively the phthalate- or triazole- unique signature. Second, we included gene expression data of MBP that was tested previously in ESC differentiation cultures [194] to study if the gene response of that experimental group grouped with the response of the remaining developmentally toxic phthalates, using the phthalate and triazoles signature. Finally, we evaluated the use of class-specific genes for identification of compound classes, by performing a leave-one-out analysis on the triazole 
gene expression data. Gene lists were identified that were regulated by a combination of two triazoles, FLU and HEX, FLU and TDI, or HEX and TDI, and were used to study triazole discrimination using PCA. For each experimental group deviation from the unexposed samples was analyzed by applying a Hotelling $T$-test to the coordinates.

Class discrimination - $\mathrm{GO}$ processes

GO identifiers (GOID) that were overrepresented in the phthalate or triazole signature, as evaluated using DAVID, were selected if the $p$-value was less than 0.05 , and the number of genes annotated to a certain GOID had to be greater than 30 and less than 300 to exclude GOID that are either too specific or too general. For each GOID that was enriched within the phthalate or triazole signature also the gene count, which is the number of genes annotated to that GOID that co-occurred in the phthalate or triazole signature, was presented. For further analyses, child terms were selected above parent terms in cases were both parent and child terms were enriched [217]. We selected additional genes annotated to the enriched GOID using AmiGO [247] and used the respective expression data to study if hierarchical clustering (Euclidean clustering, Ward linkage) could separate the phthalate-exposed from the triazole-exposed experimental groups using GeneMaths XT. The confidence value for each node in the observed clustering tree was obtained by bootstrapping the clustering 1000 times. Bootstrap values $>70$ were considered sufficient, and $>85$ were considered good.

Class discrimination - predefined gene sets

In addition, we studied the discriminative properties of previously identified gene sets that showed to be useful for identification of developmental toxicants. The expression data of the genes within these gene sets, 'Van_Dartel_heartdiff_24h' [111] and 'EST biomarker genes' [194] were used for hierarchical clustering of the experimental groups. As a negative control, the experimental groups were clustered using a gene set comprised of 200 genes not responsive to the test compounds.

\section{Results}

Compound-induced effects on gene expression in ESC differentiation cultures

On the gene expression level, the non-developmental toxicant MMP regulated only 14 genes, while MBP (at the same molar concentration) and MEHP (at a two-fold lower molar concentration) altered the expression of 171 and 803 genes, respectively. We identified 68 genes that were commonly regulated by the two developmentally toxic phthalates MBP and MEHP (Figure 2A). In terms of directionality of response, MBP and MEHP similarly impacted $67 / 68$ genes included in the phthalate signature and these 
A

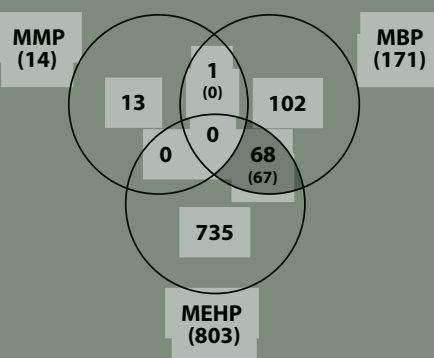

C

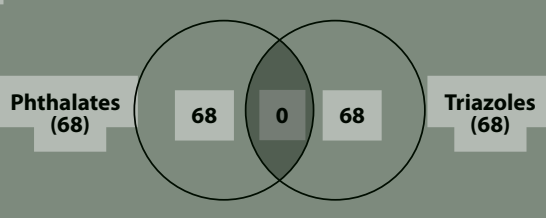

B

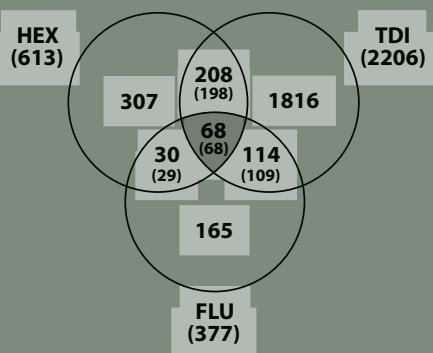

Figure 2. Venn diagrams showing the number of significantly differentially expressed genes $(p<0.001)$ per experimental group and the overlap of these regulated genes for phthalates (A) and triazoles (B). The number of genes that are regulated in common direction are presented between brackets. No genes were commonly regulated by both phthalates and triazoles (C).

67 genes were used as the phthalate signature (Figure 3A). At equipotent concentrations, the triazole compounds FLU, HEX and TDI regulated 377, 613 and 2206 genes, respectively. Sixty-eight genes were regulated by all three triazoles (Figure 2B). The expression profile of all genes included in the triazole signature was similarly affected in terms of directionality by the three triazoles (Figure 3B). These 68 genes were used as the triazoles signature. No genes overlapped between these two compound class gene signatures (Figure 2C). $P$-values corresponding to the regulation of genes within the two signatures are provided in Supplementary Table 1).

A large gene subset of both signatures, namely 46 genes (68\%) and $26(39 \%)$ for the phthalate and triazole signature respectively, was identified in the database of our earlier study [214] to be responsive in early ESC differentiation (Figure 3A and B). A large part of the genes included in the phthalate and/or triazole signature could be functionally related to the GOID 'development', 'transcription', 'response to chemical stimulus' (phthalate signature), or 'steroid metabolic process' (triazole signature).

DAVID GO Enrichment analysis revealed that the phthalate signature was enriched for 'negative regulation of cell differentiation' ( $p$-value 2.1E-4, gene count 6$)$, and 'response to inorganic substance' ( $p$-value 2.7E-3, gene count 4$)$. GO terms related to the triazole signature were enriched for'anterior posterior pattern formation' ( $p$-value 2.9E-5, gene count 7) and 'inner ear morphogenesis' ( $p$-value 2.3E-3, gene count 4). Furthermore, the $\mathrm{GO}$ term 'sterol biosynthetic process' was significantly enriched ( $p$-value 6.2E-3, gene count 3 ). 
A

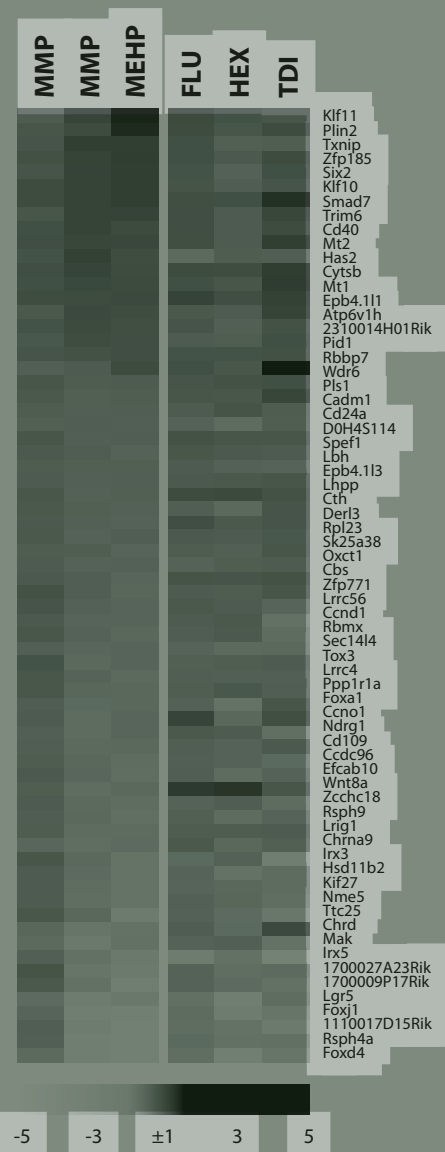

B

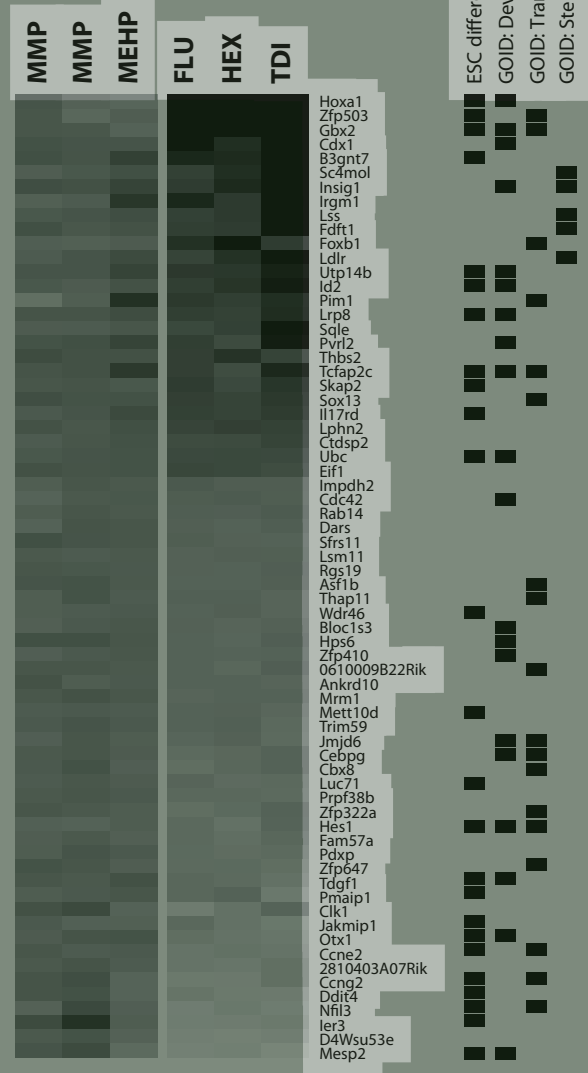

Figure 3. Heatmap of the identified phthalate signature (A) and triazole signature (B). Responsiveness of the genes to early ESC differentiation and annotation of the genes to parent terms covering enriched GOID are indicated on the right of each figure. For color figure, see Color figures section.

Class gene signatures based discrimination of phthalates and triazoles

We applied PCA using the defined phthalate and triazole signatures, respectively, to study discrimination of compound classes. Using the phthalate (Figure 4A) and triazole (Figure 4B) signatures respectively, phthalates (MBP and MEHP) appeared in distinctly separate groups from triazoles, indicating dissimilarity in gene expression profiles of these two particular gene sets between these two compound classes. In addition, 
A
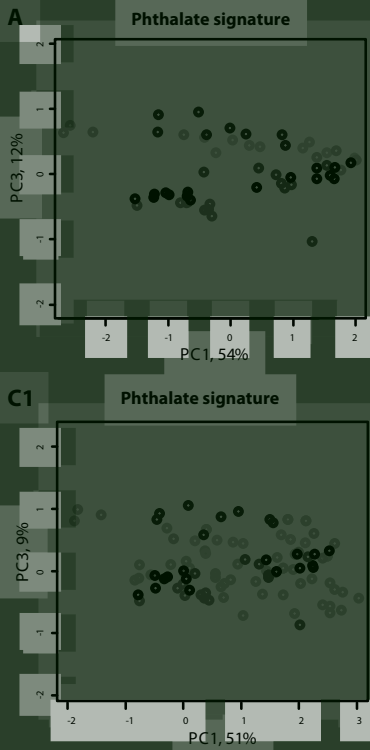

D1

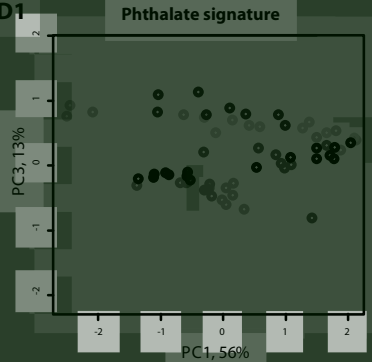

E1 Triazole signature_Leave-FLU-out

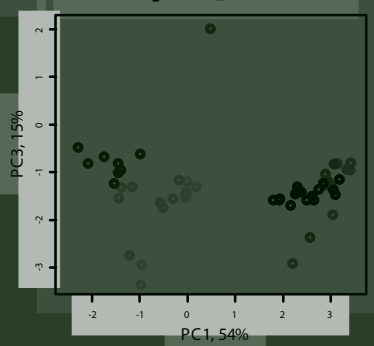

B

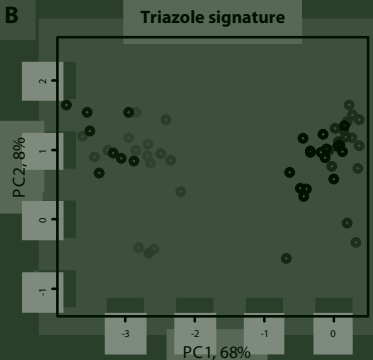

C2

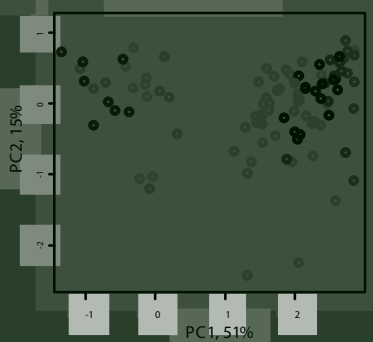

D2

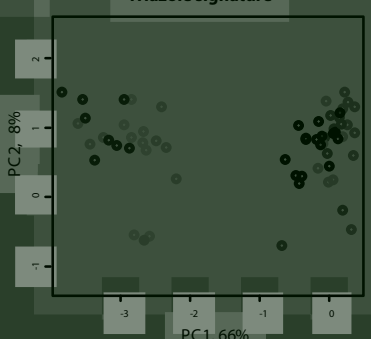

E2 Triazole signature_Leave-HEX-out

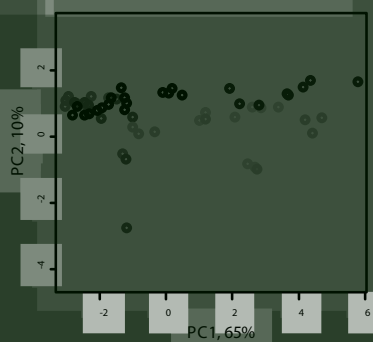

Legend

- Unexposed control

- MMP

- MBP

- MEHP

- MBP_PREV

- FLU

- HEX

- TDI

- Additional compounds
E3 Triazole signature_Leave-TDI-out

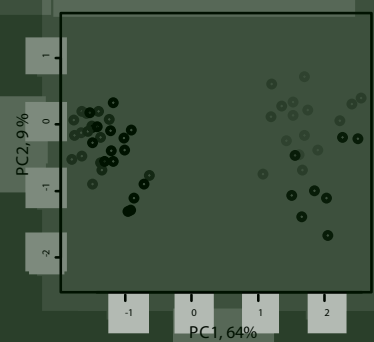

Figure 4. Discrimination of phthalates from triazoles using principal component plots of monomethyl phthalate (MMP), monobutyl phthalate (MBP), monoethylhexyl phthalate (MEHP), flusilazole (FLU), hexaconazole (HEX) and triadimefon (TDI) on the basis of the identified phthalate signature (A) and the triazole signature (B). The principal component analysis-based discrimination was validated by studying the projection of additional compounds $(\mathrm{C})$, by evaluating the position of MBP tested in our previous study (MBP_PREV, D), and by performing leave-one-out analyses (E). For color figure, see Color figures section. 
compounds within each class clustered together, indicating that the gene expression profiles within each class was similar. On the basis of the phthalate signature, cultures exposed to the non-developmental toxicant MMP were positioned closer to the negative controls than to the remaining phthalates (Figure 4A).

To validate the discriminating capacity of the phthalate- and triazole-signature for their respective class, we performed additional analyses. First, we evaluated the discrimination of the phthalate- or triazole-exposed cultures across a diverse set of compound-exposed cultures (including carbamazepine, methotrexate, methylmercury, nitrofen, saccharine, and warfarin) using PCA. Gene expression changes within the phthalate signature by triazole and phthalate compounds (Figure 4C1) indicated clear separation between MBP and MEHP on the one hand and the unexposed time-matched cultures on the other. The remaining exposed cultures were all positioned close to the control in comparison to the phthalate compounds, with the exception of one compound (carbamazepine), which overlapped with both MBP and MEHP exposed cultures and deviated from the control (corresponding $p$-values are provided in Supplementary Table 2). Evaluation of the triazole signature indicated strong differences in positioning between triazoles and unexposed time-matched cultures. The remaining compound-exposed cultures were all positioned near the control in comparison to the triazole compounds (Figure 4C2).

Second, we evaluated the position in the PCA of MBP as tested in an earlier study (MBP_PREV) [214] relative to phthalates and triazoles tested in the present study. MBP_PREV clustered together with the positive developmentally toxic phthalates MBP and MEHP, using both the phthalate and the triazole signature (Figure 4D). Finally, we used the leave-one-out approach to evaluate the usefulness of our method of gene set identification. To this end, we identified three new genes lists, each separate gene list consisted of all the genes that were commonly regulated in a combination of two triazole-exposed cultures, but not necessarily in the one that was left out. This resulted in 266, 177 and 97 genes that were commonly significantly differentially expressed in the same direction in cultures exposed to HEX and TDI, TDI and FLU, and HEX and FLU, respectively (Figure 2). Figure 4E shows that these gene lists clearly discriminated triazole-exposed cultures, and furthermore, the compound that was left out mapped closely to the other triazole-exposed samples.

Gene Ontology based discrimination of phthalates and triazoles

Figure $5 \mathrm{~A}$ and $5 \mathrm{~B}$ show the results of the hierarchical clustering of genes related to specific GOID that were enriched in either the phthalate- or triazole gene signature. Using genes associated with 'negative regulation of cell differentiation', 'anterior posterior pattern formation', and 'inner ear morphogenesis' we were able to discriminate between phthalates and triazoles. The branching of the clustering tree was mostly good in discriminating triazoles from phthalates. However, the clustering based on the 
genes associated with the GOID 'response to inorganic substance', grouped HEX and TDI together, whereas FLU was clustered with the phthalates. Clustering based on all genes annotated to the GOID 'sterol biosynthetic process' clustered the phthalate and triazole compounds separately, but TDI also appeared in a separate branch. The CON cultures clustered next to the negative developmental toxicant MMP in 4/5 of the GOID clustering analyses. Only in one clustering MBP was clustered next to the CON.

In addition, we studied overrepresentation of the class-enriched GOID in the significantly regulated gene list of the individual experimental groups. It appeared that although the identified GOID were enriched within the triazole or phthalate signature, these GOID were not necessarily enriched in each individual compound-exposed group belonging to that class (Figure 5A and 5B). For example, genes annotated to the GOID

A. GOID enriched in phthalate signature

'Negative regulation cell differentiation'(222 genes)
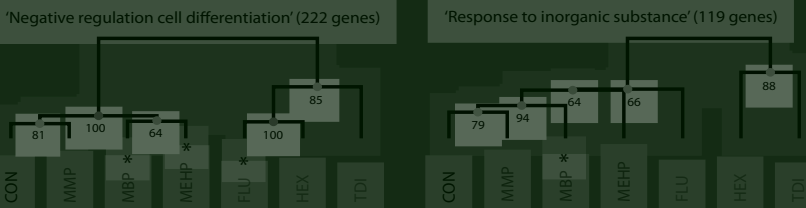

B. GOID enriched in triazole signature

'Anterior posterior pattern formation' (161 genes)
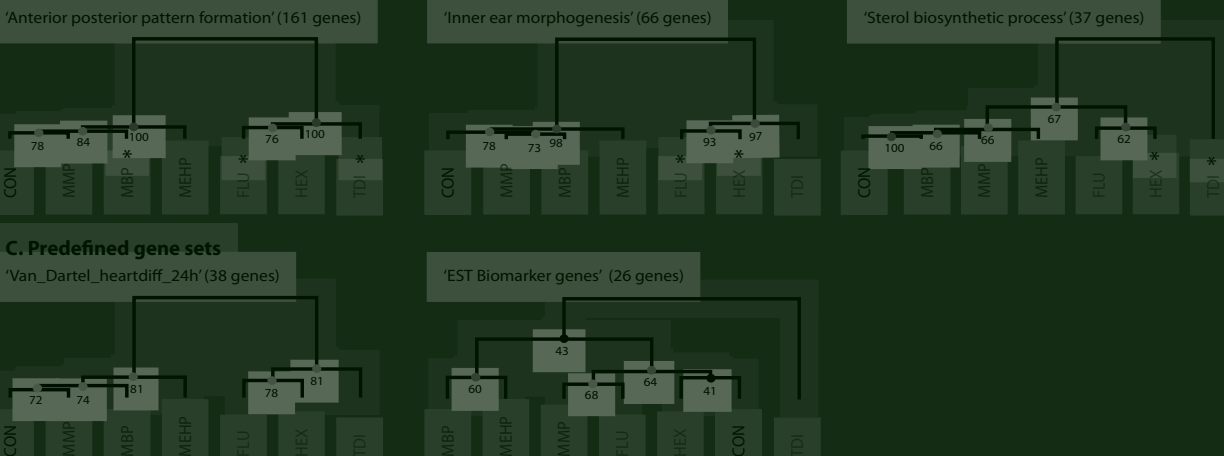

'EST Biomarker genes' (26 genes)

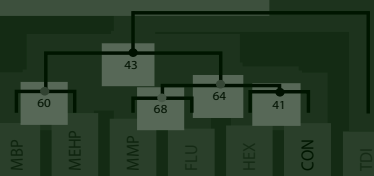

D. Negative control

'Genes not responsive to the test compounds' (200 genes)

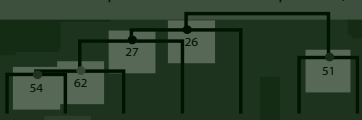

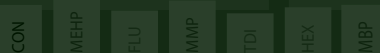

Color scale bootstrap value

$\begin{array}{lllllll}100 & 90 & 80 & 70 & 60 & 50 & 40\end{array}$

Figure 5. Hierarchical clustering of control (CON), monomethyl phthalate (MMP), monobutyl phthalate (MBP), monoethylhexyl phthalate (MEHP), flusilazole (FLU), hexaconazole (HEX) and triadimefon (TDI) using all genes annotated to gene ontology identifiers (GOID) enriched in the phthalate signature (A) or triazole signature (B), using predefined gene sets (C), and using a negative control gene set (D). Asterisks indicate significant GOID enrichment ( $p$-value $<0.05)$ within the significantly regulated genes ( $p$-value $<0.001$ ) of that compound. The color of the nodes indicates the confidence value (bootstrap value) for each node in the clustering tree. For color figure, see Color figures section. 
'anterior posterior pattern formation' were overrepresented within the 68-genes containing triazole signature, whereas studying enrichment of this GOID using the compoundspecific gene lists revealed enrichment only in the FLU and TDI group, and not HEX. Furthermore, we identified that genes annotated to the GOID 'anterior posterior pattern formation' and 'negative regulation of cell differentiation' were overrepresented within experimental groups of both compound classes (Figure 5A and 5B).

Using predefined gene sets for discrimination of phthalates and triazoles

The discriminating properties of gene sets that we defined in our recent studies are presented in Figure 5C. The 'Van_Dartel_heartdiff_24h' gene set showed a clear separation between both classes, with reliable confidence for branching. The gene set 'EST biomarker genes' correctly clustered the developmental toxicants MBP and MEHP separate from the control, and the non-developmental toxicant MMP next to the control. Within the triazole class, both FLU and HEX clustered close to the control and TDI was separately branched.

The hierarchical clustering on the basis of the genes that were not responsive to the test compounds, the negative control gene set, did not discriminate the two compound classes correctly (Figure 5D).

\section{Discussion}

Identified class signatures show successful grouping of respective class

We performed the unsupervised clustering method PCA using the identified class signatures to study discrimination of phthalates and triazoles. PCA is often used in classification studies to discriminate samples based on their similarity in gene expression response that is reflected by the relative position of samples within the PCA $[239,248]$. Although the position of the samples may differ according to data input and thereby influence discriminatory analyses, our PCA plots show robust positioning. For example, the position of the phthalate and triazole compounds in Figure $4 \mathrm{~A}$ and $\mathrm{B}$ is very similar to those in Figure $4 C 1$ and $4 C 2$, respectively, whereas in the latter two plots 48 additional samples were included.

In this study, we observed that the triazole signature clearly discriminated triazoleexposed samples from samples from all other chemical exposures. This observation was also evident using triazole signatures derived using a leave-one-out analysis, demonstrating that the discriminating property of the triazole signature was not biased by overfitting of the data. The high discriminating power of the triazole signature may in part be explained by the proposed mode of teratogenic action of triazole compounds. Triazoles are known to target Cyp enzymes involved in retinoic acid metabolism 
(reviewed in [249]). Regulation of retinoic acid levels in the embryo is critical for developmental patterning $[187,188,250]$. Interestingly, our data are in line with retinoic acid metabolism-mediated disruption of pattern formation by triazoles. First, the GOID 'anterior posterior pattern formation' was enriched in the triazole signature and in the individual gene lists of FLU and TDI. In addition, members of the retinol metabolism pathway (Crebp1, Rbp4, Rdh10) were identified within the individual gene lists of the triazole compounds (not shown).

The projection of MMP-samples in the PCA analyses towards the control samples confirms that, although MMP is similar in structure as MBP and MEHP, its effect on ESC differentiation cultures was dissimilar from that of the developmentally toxic phthalates. This finding shows that toxicity ranking of compounds within a structural class is possible and suggests that this system could be used for prioritizing compounds within a class for further testing. Furthermore, we observed that the phthalate signature did not clearly discriminate the developmentally toxic phthalates from the carbamazepineexposed samples. This could perhaps be explained by a potentially similar teratogenic mode of action of phthalate esters and carbamazepine. It has been suggested that embryonic acidification caused by accumulation of weakly acidic compounds in the basic milieu of the early mammalian embryo may mediate teratogenesis [246]. Both carbamazepine and phthalates are weakly acidic, thus such a common mode of action may play a role. However, the gene expression signature characterizing this mode of action remains unknown at the present time.

GO processes group phthalate and triazole compounds

The assembly of the two class signatures was based on the significant expression of individual genes within the present data set. In addition, the discrimination between phthalates and triazoles was also studied on the functional level using the overrepresented GOID and predefined gene sets for class discrimination.

We showed that a significant subset of genes within the identified class signatures (up to 7/68) corresponded to GOID, which were consequently identified as enriched GOID. Besides the 3-7 genes that contributed to the enrichment of the identified GOID, expression data of all genes annotated to these enriched GOID were included for the hierarchical clustering, which in some cases resulted in addition of expression data of $>150$ genes. These hierarchical clustering analyses convincingly discriminated phthalates from triazoles with corresponding confident bootstrap values.

Although the GOID were identified to be enriched within the triazole or phthalate signature, the GOID were not per se enriched in each compound-exposed group belonging to that class. This indicates that a GOID does not necessarily need to be overrepresented for successful grouping of compounds. Interestingly, we observed that the genes belonging to the GOID 'anterior posterior pattern formation' and 'negative regulation of 
cell differentiation' were also overrepresented within experimental groups of the other compound class. This finding indicates that significant enrichment of a GOID in experimental groups does not inevitably mean that these groups will cluster together, since the enrichment may be caused by dissimilar regulation of genes within the GOID or regulation of other genes present within that GOID [230].

Predefined gene sets discriminate phthalate and triazole compounds

We also performed clustering analyses using the previously identified gene sets, 'Van Dartel_heartdiff_24h' [111] and 'EST_biomarker_genes' [194] that have been shown in a recent validation study to be useful in the identification of developmental toxicants [214]. As we discussed in these previous publications, these gene sets are based on genes involved in the early stage of ESC differentiation and are consequently strongly enriched for a multitude of development-related processes.

The gene set 'Van_Dartel_heartdiff_24h' includes genes that were up-regulated in $24 \mathrm{~h}$ ESC differentiation cultures as compared to 6 and $12 \mathrm{~h}$ ESC differentiation culture. This gene set showed its usefulness for categorizing phthalate compounds for their developmental toxic potency and for discrimination between developmental toxic phthalates and triazoles exposure groups. Also the gene set 'EST biomarker genes' showed to be useful for categorization of compounds, albeit only within the phthalate class. This limitation is likely related to the approach of identification of the 'EST biomarker genes' gene set; in this gene set, all genes were included of which the expression was altered across normal ESC differentiation and which in addition were responsive to exposure of the developmental toxicants under study, including MBP [194]. Thus, the finding that this gene set correctly discriminates developmentally toxic phthalates, whereas the response to developmentally toxic triazoles appeared relatively modest, indicates that this gene set may be specified towards the differentiation-related responses observed by the compounds evaluated in that study.

Further improvement of compound discrimination is dependent on study design and analysis

The comparisons with gene expression data from our earlier studies were limited by the fact that most compounds were tested at single concentrations. To allow optimal comparison between compound-exposed experimental groups, we aimed at testing the compounds at equally effective concentrations, i.e. inhibiting ESC differentiation by approximately $50 \%$ as evaluated by morphological scoring. Unfortunately, MBP was less effective in terms of ESC differentiation inhibition in the present study, although nevertheless this exposure resulted in a gene expression response with significant commonalities to that of MEHP. This demonstrates that gene expression changes by developmental toxicants could already be identified at concentrations that did not affect ESC differentiation as evaluated by morphological scoring. This finding illustrates the 
relatively high sensitivity of gene expression changes as compared to morphological scoring in EST. However, testing MBP at a 50\% morphological effect concentration may have further enhanced the specificity of the phthalate signature.

The importance of compound concentration for the gene expression response was also evident from additional cluster analyses using gene expression data of FLU tested in a concentration series that was available from a previous study [235]. Whereas the cluster analysis based on GOID and the initial data (FLU tested at $5.4 \mu \mathrm{M}$ ) resulted in a misclassification of FLU for the GOID 'response to inorganic substance', using gene expression data of FLU tested at $18 \mu \mathrm{M}$ resulted in a perfect discrimination of phthalates from triazoles (Supplementary Figure 1). Moreover, also the clustering of the 'sterol biosynthetic process' resulted in an improved discrimination of the two studied classes. We and others have shown that compound concentration has significant effects on the gene expression response [49, 225, 251], and therefore we suggested studying compounds in a concentration-response design to optimize both the identification of potential developmental toxic properties and the discrimination between compound classes.

Even with the limited compound set evaluated in this study, it is apparent that each compound produced its own unique expression profile and that similarities in profiles between compounds can indicate similarities in toxic mechanisms. However, our study does not provide decision rules for compound classification, since this requires supervised models, such as support vector machines and logistic regression, based on a large data set. Studies have been described that use a large dataset to predict toxicological outcome and delineate mechanisms of action in a supervised manner [239, 252]. Additionally some studies make use of reference toxicogenomic databases to allow these gene signature comparisons and improve the predictability of their study [238]. So far, the ToxRefDB has been the most advanced database in reproductive and developmental toxicology that covers both in vivo and in vitro data of 474 chemicals [253]. Unfortunately, a toxicogenomic database that includes a substantial number of developmental toxicants is not (yet) available. Nevertheless, the discriminative gene sets identified in the present study can be used as starting points for more advanced classification studies using ESC differentiation.

In conclusion, we have demonstrated the ability to distinguish between two structural classes of compounds with distinct modes of action in the EST using toxicogenomic data. Transcriptomic responses proved to be more sensitive than classical morphological end point evaluation Studies examining concentration and time response will be critical in optimizing the discrimination of classes of compounds using toxicogenomics. The present study serves as an example for the use of compound clustering using gene expression data of exposed differentiating ESC that may be useful for prioritizing compounds for further testing and for tailoring toxicological screening strategies. 


\section{Acknowledgements}

This work was supported by the Dutch technology society foundation STW [grant MFA6809]. The sponsor was not involved in the design and conduct of the study; collection, management, analysis, and interpretation of the data; and preparation, review, or approval of the manuscript.

\section{Supplementary data}

Supplementary data associated with this article can be found at doi:10.1016/j.toxlet.2010.12.019. 
Jeroen L.A. Pennings, Dorien A.M. van Dartel, Joshua F. Robinson, Tessa E. Pronk, Aldert H. Piersma

Toxicology (2011) 284:63-71 
CHAPTER 9

Gene set assembly for quantitative prediction of developmental toxicity in the Embryonic Stem cell Test 


\begin{abstract}
The Embryonic Stem cell Test (EST) is an in vitro method for predicting developmental toxicity based on compound-induced inhibition of embryonic stem cell (ESC) differentiation. We previously described how gene expression analysis in the EST can be used to describe normal ESC differentiation as well as identify compound developmental toxicity, by means of our 'differentiation track' algorithm. In this study, we combined raw data from our three previous studies in a new integrated analysis, to identify a gene set that allows for improved prediction. By evaluating predictions of 100,000 randomly selected gene sets, we identified which genes contribute significantly to the prediction reliability. By additional cross-validation, we identified a set of 52 genes that allows for improved prediction of toxicity. The correlation between the predictions using this gene set and the magnitude of the EST end point was 0.85 , and the gene set predicted developmental toxicity with $83 \%$ accuracy (area under the curve $89 \%$ ). If compounds with ineffective data because of a too low tested concentration or too much variation between samples were excluded, even $100 \%$ accuracy could be reached based on 15 compounds. This novel gene set consists mainly of genes involved in the stem cell differentiation or other developmental processes. We expect that this set can be of use in future studies aimed at improving the EST for risk assessment, thus making a next step towards regulatory implementation of this method.
\end{abstract}




\section{Introduction}

Current regulatory guidelines for toxicological hazard identification require large numbers of experimental animals. Animal use is especially high in the area of reproductive and developmental toxicity testing [119]. For this reason, numerous efforts are investing into developing alternative testing methods to achieve reduction, refinement and replacement of animal testing to predict reproductive and developmental toxicity. As a result, many alternatives testing methods have been developed in recent decades [254], although none have yet obtained regulatory acceptance. The ECVAM validation study of three alternatives has been the largest effort in this area so far [20], but still regulatory implementation has lagged behind, which is due to remaining uncertainties regarding applicability domains and predictive capacities of the tests involved [23, 170]. Improvements in these areas are clearly necessary to increase acceptance and implementation of these tests in regulatory toxicology.

Of the three ECVAM validated alternatives for developmental toxicity, the Embryonic Stem cell Test (EST) is the only one which is completely animal-free [19]. The EST uses a continuous murine embryonic stem cell (ESC) line, which can be induced to differentiate into contracting cardiomyocytes, via the formation of embryoid bodies (EB). After ten days of culture, the extent of differentiation is evaluated microscopically, and compounds inhibiting this differentiation process are considered as potential developmental toxicants.

Despite its promise as an alternative assay [170], several disadvantages of the current EST protocol exist, including the long duration of culture and subjective scoring for the presence of contracting cardiomyocytes. Recent studies suggest that molecular biological approaches, such as transcriptomics, may help to improve the prediction accuracy of this test system [111, 255, 256]. Furthermore, these studies provide more detailed information regarding mechanisms of action in relation to concentration [235] and time [177], thus allowing further optimization of this testing method both in terms of applicability domain as well as for developmental toxicity prediction.

As part of the analysis of transcriptomic data, Principal Component Analysis (PCA) has been applied to describe gene expression changes during embryonic development $[153,232]$ and stem cell development $[177,231]$. These studies found that phases in the developmental process appear in a PCA visualization as a chronological differentiation track. In previous studies from our laboratory, we described that exposure of ESC to developmentally toxic compounds during early cardiomyocyte differentiation (day 3-5 of culture), results in a deviation from the ESC 'differentiation track' (van Dartel et al., 2010d). In other studies, we described two gene sets that can be used to define this 'differentiation track' and can also be used to identify deviation form this track by exposure to developmental toxicants $[111,194]$. The 'Van_Dartel_Heartdiff_24h' gene set includes 
38 genes that were all up-regulated at $24 \mathrm{~h}$ ESC differentiation compared to earlier time points. This gene set was observed to be down-regulated by the compound monobutyl phthalate, thereby showing its value in developmental toxicity identification. The 'EST_biomarker_genes' gene set includes 26 genes that were up- or down-regulated during normal ESC differentiation (0-48 h) and also responsive to various developmental toxicant exposures. An evaluation study [214] showed that a 'differentiation track' algorithm using these gene sets can lead to up to $83 \%$ prediction accuracy in a group of 18 compounds. In addition, we found that the significance of 'differentiation track' deviation not only depends on the compound, but also on the concentration that was being tested [235]. Because this also applies to the EST end point of cardiomyocyte differentiation inhibition, it can be deduced that a more significant track deviation is predictive for a higher magnitude of the inhibition at the cardiomyocyte differentiation end point. Each of these initial studies individually provided information on the possible use of PCA in defining normal and altered differentiation and the application of gene sets in predicting toxicity. In the present study, the cumulative microarray data and toxicological information from these earlier studies have been combined in a new integrated analysis yielding a novel gene set that is investigated for its predictive ability on the tested compounds in the EST. The aim was to assemble a gene set that would allow for more accurate developmental toxicity prediction in terms of classification accuracy and/or more quantitative agreement between 'differentiation track' deviation and the morphological differentiation inhibition end point. Especially this latter aspect would be welcomed to demonstrate the applicability of the EST for quantitative data in human risk assessment.

In this study, we present an approach based on random sampling and testing of gene sets to evaluate which genes give good performance for toxicity prediction in the EST with the 'differentiation track' algorithm across multiple datasets. Using this approach, combined with cross-validation, we derive a gene set that gives better prediction performance in both aspects mentioned.

\section{Methods}

In three previous publications from our group [194, 214, 235], methods and data regarding culture conditions, compound exposures, assessment of differentiation inhibition, and gene expression profiling have been described in full detail. Microarray data obtained in those studies are available at ArrayExpress (http://www.ebi.ac.uk/arrayexpress) under accession numbers E-TABM-903, E-MTAB-300, and E-TABM-1027.

Unless mentioned otherwise, $\mathrm{R}$ was used for all calculations described in this study. The algorithm for calculating deviation from the 'differentiation track' has been de- 
scribed previously [177, 194]. Briefly, starting from whole-genome normalized data, gene expression data for a selected subset of genes are log-transformed, and data for unexposed samples at $0 \mathrm{~h}, 24 \mathrm{~h}$, and $48 \mathrm{~h}$, together with compound treatment samples at $24 \mathrm{~h}$, are used in a PCA. Coordinates along the first and second principal component are calculated for each sample. Deviation of compound-exposed samples from the 'differentiation track' is analyzed by applying a Hotelling T-test to these coordinates compared to those of the time-matched control cultures.

To determine which genes make reliable indicators for 'differentiation track' deviation, we used a five-step approach. First, the data set was restricted to those genes that showed significant differences in expression between the controls at $0 \mathrm{~h}, 24 \mathrm{~h}$, and $48 \mathrm{~h}$ $(p<0.001$, Fold Ratio (FR) $>1.25)$. This reduced the number of genes in the data set from 20979 (all annotated genes on the microarray) to 5601. In the second step, 100,000 randomly selected sets of 30 genes each were taken from the data set (so that each gene was sampled on average 536 times). The amount of 30 genes per set was chosen to be in accordance with the approximate size of the two previously identified gene sets [111, 194]. For each gene set, we calculated the $p$-value for 'differentiation track' deviation for each 24 h-exposed experimental group using the data of this group plus data of the three unexposed experimental groups, sampled at $0,24,48 \mathrm{~h}$ of ESC differentiation, derived from the same study. Unexposed samples were used in the analysis as a reference group with (by definition) no differentiation inhibition. For each set, the calculated $p$-values for the various compound exposures were compared to the corresponding ESC differentiation inhibition by calculating the Spearman (rank-based) correlation. A low $p$-value for 'differentiation track' deviation is expected to correspond to a high degree of ESC differentiation inhibition, therefore genes of a gene set that have a low correlation

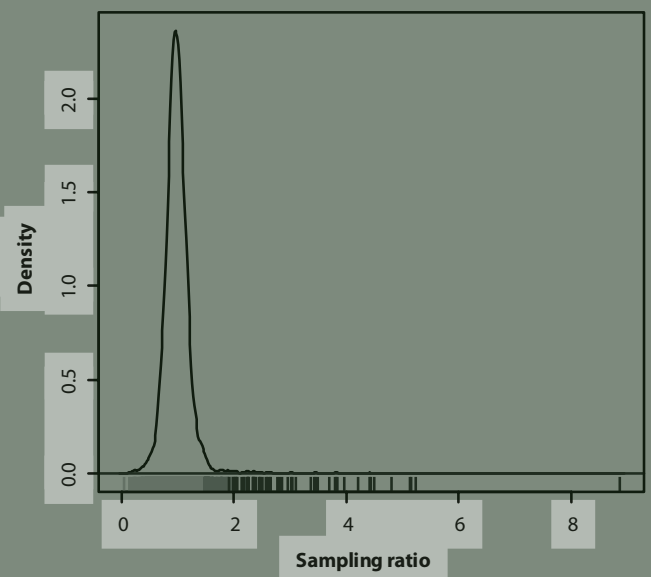

Figure 1. Vertical lines below the distribution curve indicate significance status of individual genes. Grey, not significant; dark grey, significant (FDR 5\%) but not cross-validated; black, significant and cross-validated. 
are expected to be most predictive. The genes in the gene set were scored as a hit if the correlation for a gene set was less than -0.7571 , which corresponds to the $10^{\text {th }}$ percentile, based on a pilot run with 10,000 samplings. After the computations for 100,000 gene sets were finished, it was determined how many times each gene was sampled and how many times it had scored a hit (i.e. exceeded the threshold). This latter value was compared to the expected number of hits (i.e. 10\% of the number of samplings). Because for most of the genes this scoring ratio (actual hits / predicted hits) followed a Gaussian distribution (Figure 1), this distribution was used to determine which genes had a significantly higher ratio, at a False Discovery Rate (FDR) of 5\%. All identified genes were combined in a set (Pennings_Diff_Correlation) and track deviation $p$-values were calculated and compared to those calculated with the two previously described gene sets.

Fourth, a leave-one-out cross-validation (LOOCV) was carried out. This comprised of repeating the above procedure with each time one of the treatment groups left out, and determining which genes are found significant at an FDR of $5 \%$. These cross-validation gene sets were used to calculate the $p$-value for the left out group. Finally, those genes in the Pennings_Diff_Correlation set that were confirmed by all cross-validations were combined in a restricted gene set (Pennings_Diff_Crossval).

Gene sets were evaluated for toxicity identification by means of the 'differentiation track' algorithm, using 18 compound-exposed cultures used in the biomarker discovery and validation study [194, 214]. Compounds were considered developmentally toxic if they showed significant deviation from the 'differentiation track' $(p<0.05)$ and as nontoxicants otherwise. For additional evaluation, Receiver Operating Characteristic (ROC) curves and their underlying Area Under the Curve (AUC) were obtained in R. Visualization and hierarchical clustering of gene expression data was carried out using GeneMaths XT (Applied Maths, St-Martens-Latem, Belgium). Enrichment for Gene Ontology and other functional terms was determined using the DAVID Bioinformatics Resource website (http://david.abcc.ncifcrf.gov) [257]. Additionally, enrichment for other custom functional gene sets such as e.g. described in [258] was determined via an in-house developed R application.

To determine how genes or proteins in the Pennings_Diff_Correlation set act together in ESC differentiation, we collected a list of mutual gene or protein interactions via a query at STRING [259] (http://string.embl.de). Interactions were included in the list if they had high confidence scores ( $>0.7$ ) in mouse, high confidence scores in human, or medium confidence scores $(>0.4)$ in both. This list was further supplemented with curated interactions that were found through building a gene interactions network in MetaCore (www.genego.com). After redundancies in the list were removed, the interactions were visualized in Cytoscape [127]. 


\section{Results}

Assembly of a novel predictive gene set

With the methods described, we identified 132 genes that are significantly enriched among gene sets that show a strong correlation between 'differentiation track' deviation and ESC differentiation inhibition. These genes were combined to form a novel gene set that was named 'Pennings_Diff_Correlation' (Supplementary Table 1). Using the 'Pennings_Diff_Correlation' gene set, we observed strong negative correlation (-0.904) between 'differentiation track' deviation of compound-exposed experimental groups and ESC differentiation inhibition (Table 1). As could be expected, weaker correlations were observed with the previously established gene lists EST_biomarker_genes set $(-0.620)$ and Van_Dartel_Heartdiff_24h set $(-0.714)$, respectively, that were based on more limited datasets.
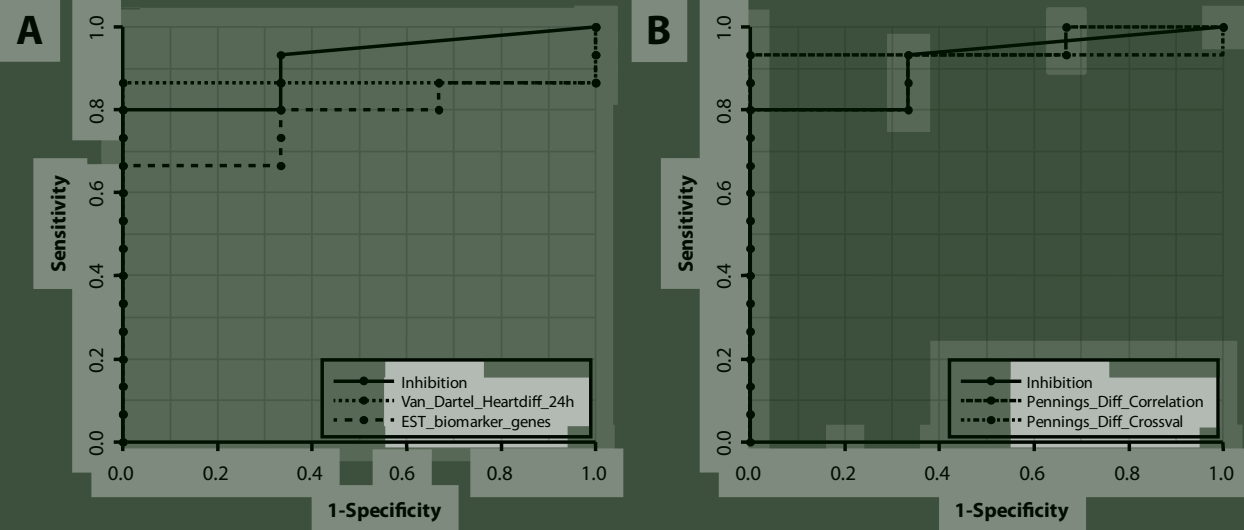

Figure 2. ROC curves are given for the magnitude of the cardiomyocyte inhibition ("inhibition") and predictions made using the differentiation track algorithm. A, previously reported gene sets. B, gene sets reported in this study.

We also determined the classification performance for the 18 previously described exposures by comparing the obtained $p$-values in a ROC curve (Figure 2). The resulting AUC is 0.956 for the Pennings_Diff_Correlation set, compared to 0.778 for the EST_biomarker_genes set or 0.867 for the Van_Dartel_Heartdiff_24h set. Again as expected, the Pennings_Diff_Correlation set showed in improved developmental toxicity prediction as compared to previously obtained gene sets. When the previously used $p$-value of 0.05 was used as a cutoff between toxicants and non-toxicants, the overall number of misclassifications for the 18 exposures tested in the biomarker discovery [194] and validation study [214] was 3 with the Pennings_Diff_Correlation set, 3 with the Van_Dartel_Heartdiff_24h set, and 4 for the EST_biomarker_genes set. Thus, using this $p$-value, 


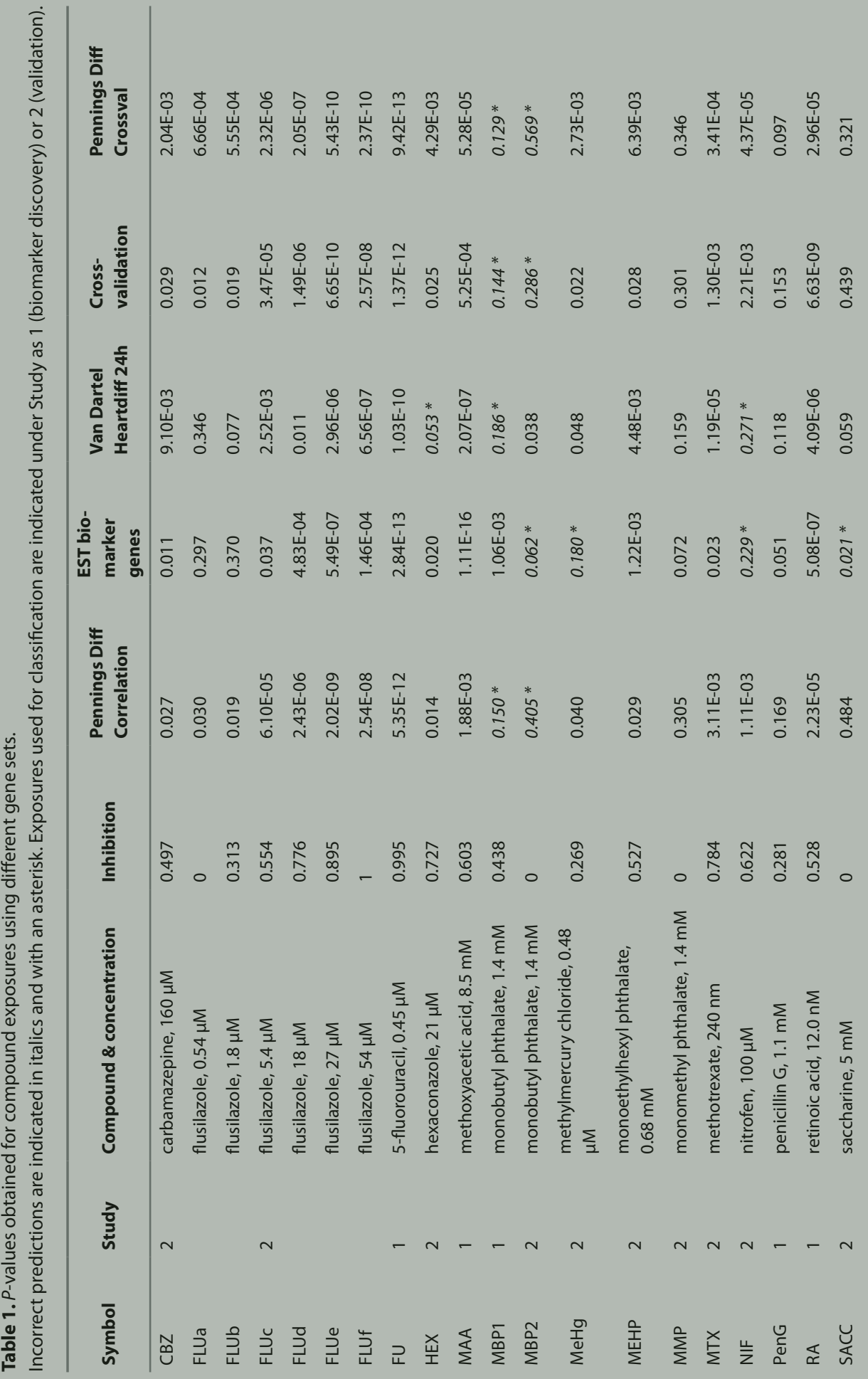




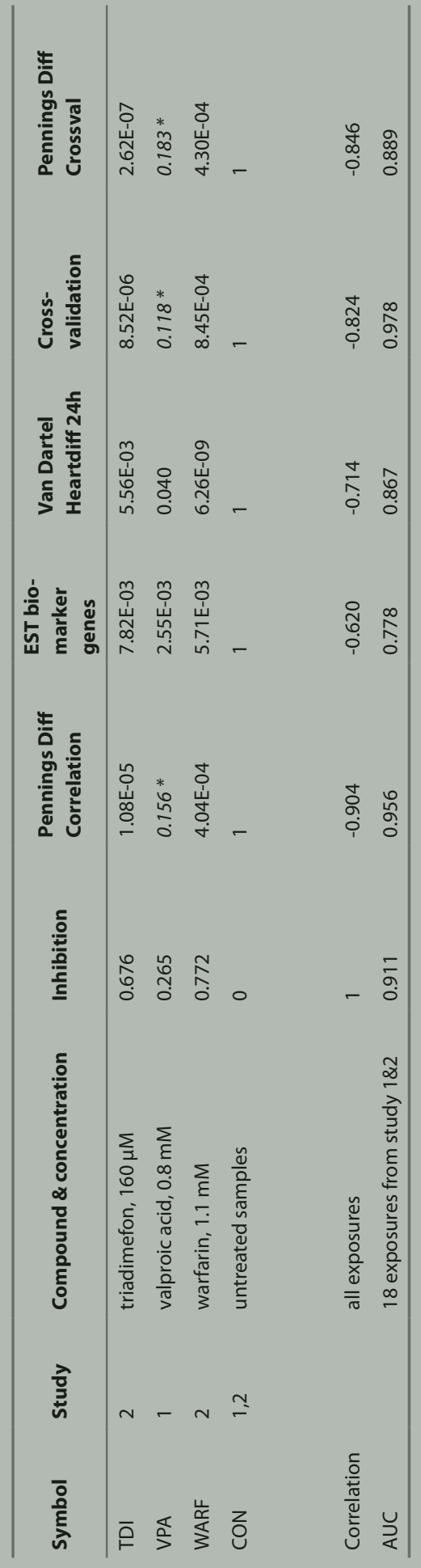


the improvement in classification AUC is not reflected in a higher number of correct predictions compared to the Van_Dartel_Heartdiff_24h set.

Biological characterization

Hierarchical clustering on gene expression data across normal ESC differentiation (unexposed groups) for the 132 genes in the Pennings_Diff_Correlation set revealed four clusters with different expression timing patterns (Figure3 (inset)). Clusters A-D peaked at different culture time points, namely at $0 \mathrm{~h}$ (labeled A), 24h (B), 24-48h (C), and 48h (D) (Supplementary Table 1). Thus, the set consists of genes of which the expression peaks cover the entire time period studied.

A considerable number of the 132 genes were found to function in development related processes. According to $\mathrm{GO}$ annotation, there are 62 genes annotated as involved in development ( $p$-value = $3.7 \mathrm{E}-20$ ), including 34 involved in embryonic development ( $p$-value 1.9 E-19). Most notable in this respect is the finding that the set contains nine Hox genes and seven other genes with homeobox domains (Alx3,Cdx2,Gbx2, Lhx1, Otx1, Otx2, Pou3f1). The highest ranking gene, Myl7, represents an example of more specifically cardiac-associated genes. The functional relevance of the 132 genes was further corroborated by the presence of 45 genes which are also included in the ESC-differentiation co-regulation network which we recently constructed based on a collection of relevant literature [258]. Finally, via STRING and MetaCore databases queries, we identified 65

-

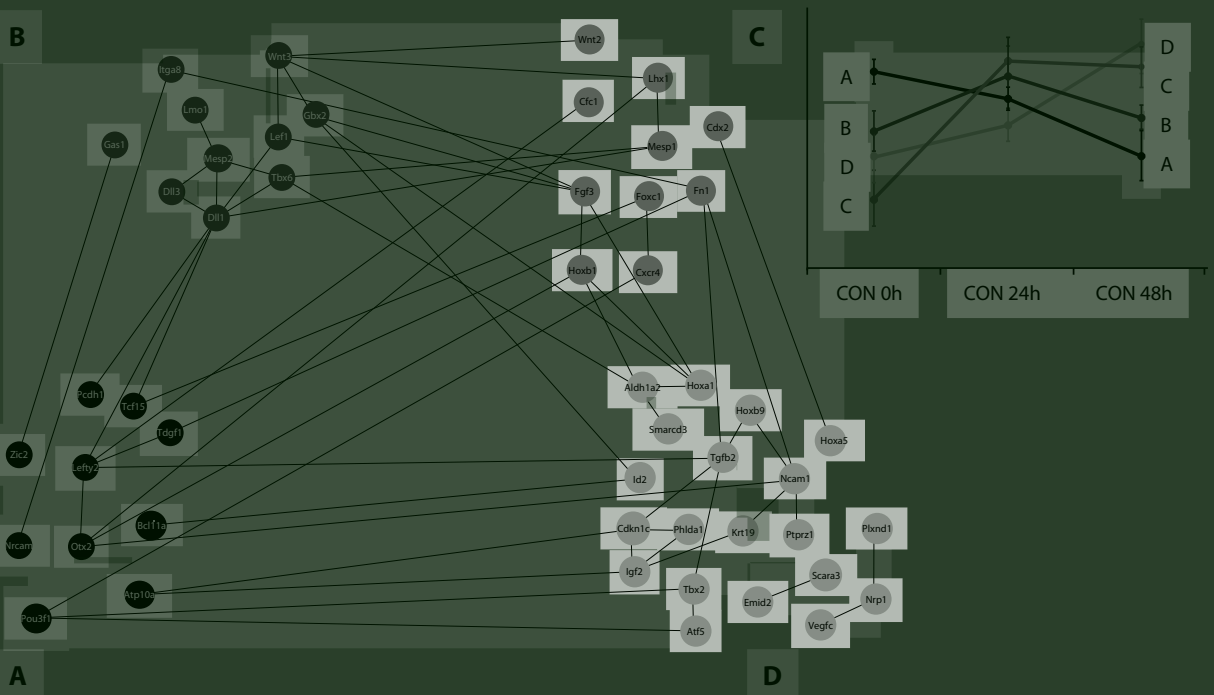

Figure 3. Gene or protein interactions found in the STRING and MetaCore databases are indicated as a connection between gene nodes. Gene node colors indicate the expression cluster to which a gene belongs (cluster A-D, see inset). 
gene or protein interactions among 50 of the 132 genes in the Pennings_Diff_Correlation set (Figure 3 ).

When gene expression and interaction data are shown together (Figure 3) it can be seen that many of the interactions are between genes that share a similar time expression trend. More precisely, there are 30 interactions within time clusters $(1.24 \%$ of all possible within-cluster interactions) versus 35 interactions between time clusters ( $0.56 \%$ of all possible between-cluster interactions). Thus, within-cluster interactions are relatively nearly 2.5 times more frequent, which is in agreement with the premise that cooperating genes should be similarly expressed. Also, the number of within-cluster interactions is highest in the genes which are expressed at later timepoints, consistent with a cellular system that becomes more specialized to a specific function. Within the network, the genes with most interactions (e.g. DII1, Fgf3, Lefty2, Ncam 1, Tgfb2, Wnt3) are all known as developmental genes.

Cross-validation

To test the robustness of our results, both with regard to the gene set obtained as well as the predicted $p$-values per compound exposure, we cross-validated the analysis using a LOOCV. When $p$-values were calculated for the exposure that was left out of the cross-validation, the values obtained were generally comparable with those for the Pennings_Diff_Correlation gene set (Table1), with only the $p$-value for retinoic acid differing by more than an order of magnitude. The overall correlation for the LOOCV predicted values with the magnitude of EST differentiation inhibition was -0.824 .

Of the 132 genes in the initial gene set, 87 were confirmed in the majority, and 52 in all of the cross-validations. We named the latter set of genes 'Pennings_Diff_Crossval' (Table2). Generally speaking, these are mainly the genes with higher scoring ratios, and correspondingly lower FDRs in the Pennings_Diff_Correlation set (Supplementary Table1, Figure 1). Using this set, $p$-values for compound-induced 'differentiation track' deviation were calculated, and the correlation between these significance values and ESC differentiation inhibition was -0.846. In addition, the AUC based on 18 previously tested exposures was 0.889 (Table 1). Classification on the 18 previously tested exposures resulted in 3 misclassifications (MBP1, MBP2, VPA) (Table 1), which were the same as for the Pennings_Diff_Correlation set.

The Pennings_Diff_Crossval set also showed functional enrichment for developmental (28) and embryonic development (16) genes, as well as for 22 genes in the ESCdifferentiation co-regulation network. Also, 21 of the 50 genes with mutual interactions (Figure 3) are also included in the Pennings_Diff_Crossval set. As a relative percentage, these figures are between 2 and $9 \%$ higher than for the Pennings_Diff_Correlation set. 
Table 2. Genes in the Pennings_Diff_Crossval set.

\begin{tabular}{|c|c|c|c|}
\hline Gene symbol & GenelD & Scoring ratio & Cluster \\
\hline 1700013H16Rik & 75514 & 2.504 & A \\
\hline Bcl11a & 14025 & 2.003 & $A$ \\
\hline Lefty2 & 320202 & 4.806 & $A$ \\
\hline LOC100045988 & 100045988 & 2.239 & $A$ \\
\hline Pmaip1 & 58801 & 2.269 & A \\
\hline Tdgf1 & 21667 & 3.801 & $A$ \\
\hline Zic2 & 22772 & 2.035 & $A$ \\
\hline 3110040M04Rik & 73176 & 3.842 & B \\
\hline Adam19 & 11492 & 2.458 & B \\
\hline Adamts9 & 101401 & 1.992 & B \\
\hline Cbln1 & 12404 & 3.429 & B \\
\hline Chst7 & 60322 & 2.591 & B \\
\hline Dclk1 & 13175 & 2.267 & B \\
\hline Dlc1 & 50768 & 2.394 & B \\
\hline Dll1 & 13388 & 2.350 & B \\
\hline DII3 & 13389 & 4.210 & B \\
\hline Egln3 & 112407 & 3.360 & B \\
\hline Gas1 & 14451 & 2.447 & B \\
\hline Gbx2 & 14472 & 2.247 & B \\
\hline Gm239 & 237558 & 2.183 & B \\
\hline Grrp1 & 72690 & 5.127 & B \\
\hline Itga8 & 241226 & 4.444 & B \\
\hline Mesp2 & 17293 & 2.862 & B \\
\hline S1pr5 & 94226 & 2.389 & B \\
\hline Tasp1 & 75812 & 2.139 & B \\
\hline Tbx6 & 21389 & 2.022 & B \\
\hline Anxa2 & 12306 & 3.113 & $C$ \\
\hline Aplnr & 23796 & 3.966 & $C$ \\
\hline Asb4 & 65255 & 3.704 & $C$ \\
\hline Cxcr4 & 12767 & 2.660 & C \\
\hline Fgf3 & 14174 & 2.560 & $C$ \\
\hline Foxc1 & 17300 & 3.814 & C \\
\hline Frzb & 20378 & 5.162 & $C$ \\
\hline Hoxb1 & 15407 & 2.171 & $C$ \\
\hline Lhfp & 108927 & 3.461 & $C$ \\
\hline Lhx1 & 16869 & 4.495 & C \\
\hline Mesp1 & 17292 & 5.244 & C \\
\hline Myl7 & 17898 & 8.865 & $C$ \\
\hline
\end{tabular}




\begin{tabular}{llll}
\hline Gene symbol & GenelD & Scoring ratio & Cluster \\
\hline Rasgrp3 & 240168 & 2.767 & $\mathrm{C}$ \\
Rbm24 & 666794 & 3.036 & $\mathrm{C}$ \\
Arg1 & 11846 & 2.964 & $\mathrm{D}$ \\
AU015836 & 385493 & 2.809 & $\mathrm{D}$ \\
Cpm & 70574 & 2.336 & $\mathrm{D}$ \\
H19 & 14955 & 2.057 & $\mathrm{D}$ \\
Hoxa1 & 15394 & 2.261 & $\mathrm{D}$ \\
Hoxd1 & 15429 & 2.472 & $\mathrm{D}$ \\
Id2 & 15902 & 2.620 & $\mathrm{D}$ \\
Plac1 & 56096 & 1.906 & $\mathrm{D}$ \\
Runx1t1 & 12395 & 3.013 & $\mathrm{D}$ \\
Scara3 & 219151 & 2.660 & $\mathrm{D}$ \\
Tgfb2 & 21808 & 3.489 & $\mathrm{D}$ \\
Zfp503 & 218820 & 4.409 & $\mathrm{D}$ \\
\hline
\end{tabular}

Toxicological response

A comparison of gene expression responses due to the various exposures (Figure 4) shows that most genes in the Pennings_Diff_Crossval set did not respond by a doseresponse consistent up- or down-regulation upon compound exposure. Instead, many genes showed either up- or down-regulation depending on the compound, indicating that toxicological effects are mediated through various mechanisms. In spite of this, some similarities could be found in a subset of the genes for some of the compounds. A similar gene expression profile could for example be seen for the triazoles (hexaconazole, triadimefon, flusilazole), which was especially characterized by induction of several Hox-genes. This characteristic profile was not found among the other compounds, with exception of retinoic acid. Additionally, similarities can be seen between warfarin, methotrexate, and methyl mercury in a number of genes in cluster $\mathrm{C}$, such as Myl7.

\section{Discussion}

In a series of previous papers, our laboratory described the use of a 'differentiation track' algorithm to study responses to compound exposure within a continuously changing biological system of ESC differentiating in vitro. This approach was built upon the knowledge that although developmental toxicants may cover a wide range of possible mechanisms of action, many of them share the ability to modulate ESC differentiation in the EST protocol by causing aberrant gene expression during critical developmental stages. Therefore, rather than looking for specific up-or down-regulation within a limited 


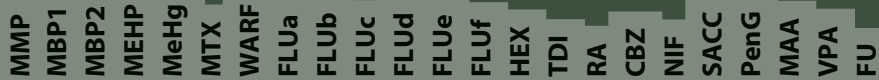
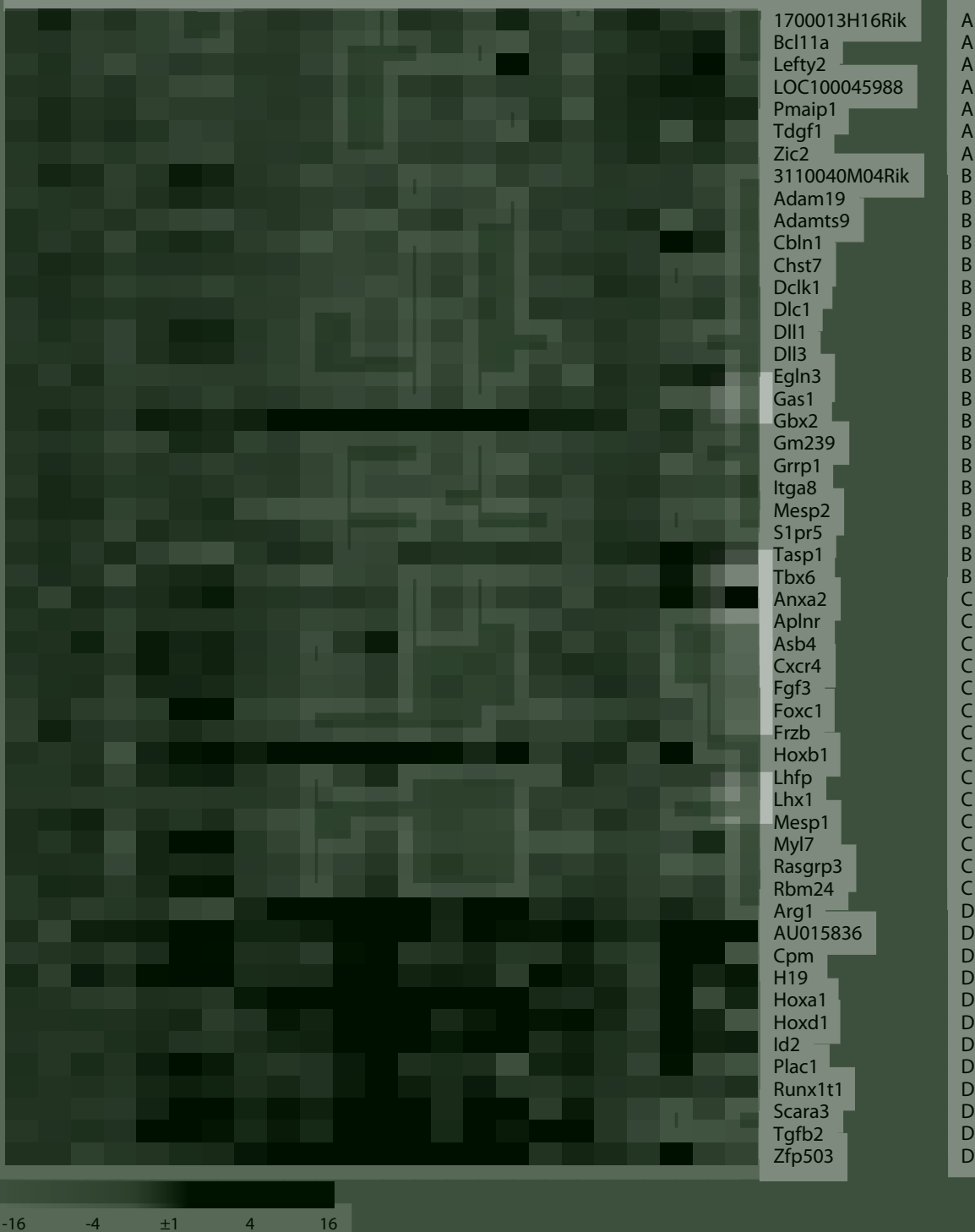

Figure 4. Expression response to compound exposure for genes in the Pennings_Diff_Crossval set. Samples are ordered based on compound class or profile similarities.

number of biomarker genes, the 'differentiation track' algorithm determines the degree of deviation from the normal 'differentiation track' as a multivariate measure (i.e. distance) based on a larger number of genes. This is done by means of a PCA that includes control groups at 0,24 , and $48 \mathrm{~h}$ plus a compound-exposed group at $24 \mathrm{~h}$, so that the 
PCA shows changes in time due to normal ESC differentiation as well as compoundinduced changes to this differentiation.

Based upon a proof-of-principle and a validation study [194, 214], the use of our 'differentiation track' algorithm in hazard identification is sufficiently reliable to investigate if it can be further improved with regard to other aspects of the EST. One such aspect is to improve the correlation between the $p$-value for 'differentiation track' deviation and the quantitative EST phenotypical end point, i.e. the magnitude of cardiomyocyte differentiation inhibition. This will probably lead to a better prediction performance, but moreover it will allow using the data in a more quantitative manner, which will certainly help accept its use in human risk assessment [192, 240, 260].

The desire for a better correlation to a quantitative end point led us to assemble a novel gene set aimed at meeting these requirements. The algorithm used was written specifically for this study, but uses several elements (e.g. random sampling of genes, scoring them according to their performance, cross-validation) which are also used in more generally applicable methods for classification and biomarker selection, especially RandomForest [261]. As anticipated, the analysis resulted in a gene set with improved correlation with the EST end point, as well as the in vivo end point, compared to earlier gene sets. In addition, the classification based on data from 18 compounds previously tested (at concentrations that were calculated on the basis of historic data to inhibit cardiomyocyte differentiation with 50\% compared with control cultures), also becomes better, with AUC values being 0.956 and 0.889 for the Pennings_Diff_Correlation and the Pennings_Diff_Crossval sets, respectively.

When a cutoff $p$-value of 0.05 is used, both of the new sets led to 3 misclassifications, equal to the number for the Van_Dartel_heartdiff_24h set, and one less that that for the EST_biomarker_genes set. More specifically, the two Pennings_Diff sets differed from the other two sets in that nitrofen (62.2\% inhibition) was correctly classified, whereas the reverse was true for valproic acid ( $26.5 \%$ inhibition). Although this implies that one correct compound (mis)classification is exchanged for another, the finding that the compound with more inhibition now became classified correctly is in agreement with our aim of a better overall quantitative prediction performance. Moreover, the valproic acid-exposed samples show large variation (Figure 5) which is over an order of magnitude outside the range of the sample variations found for all other exposures (Figure 5), which has caused this compound to be not significantly different from the controls. Regarding the other two misclassifications, it is remarkable that they both occurred for monobutyl phthalate. This compound gives malformations at relatively high concentrations and is therefore considered a weak developmental toxicant [91, 92, 122]. Moreover, monobutyl phthalate only affected ESC differentiation in one out of two studies, and data from these two studies did not lead to consistent predictions using the Van_Dartel_Heartdiff_24h and EST_biomarker_genes set (Table 1), which is 

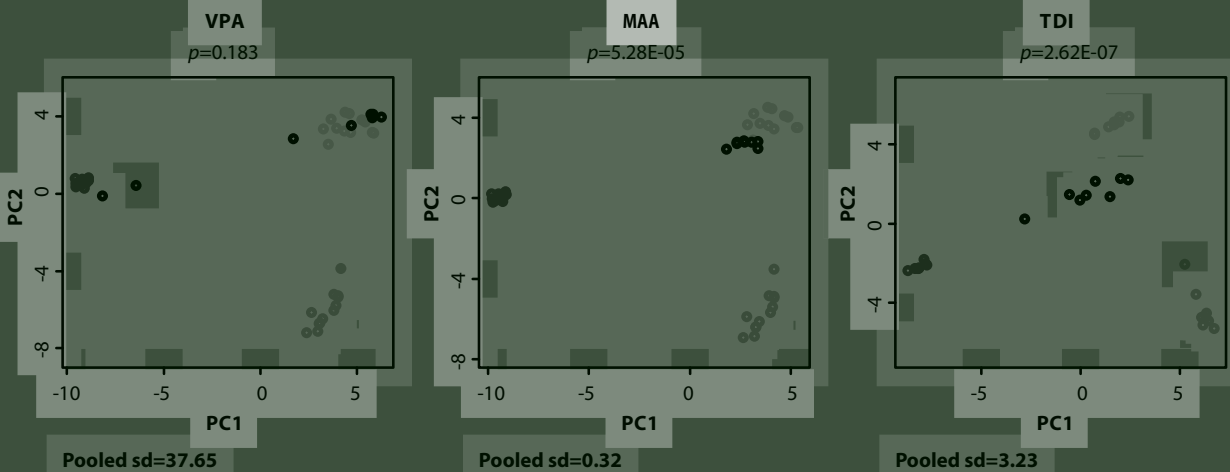

Figure 5. Examples of differentiation tracks, with different kinds of variation among toxicant-exposed samples (black circles).

probably caused by the very limited induction of gene expression changes by MBP as observed in our previous studies as well as in the present study (Figure 4). This suggests that if MBP had been dosed at a higher concentration that led to more differentiation inhibition as well as gene expression changes, it might have been predicted correctly as developmentally toxic

Taken together, if variability among samples could be reduced and test compound concentration would be optimized, predictability of the Pennings_Diff_Crossval gene set could rise to $100 \%$ for the compounds tested. These aspects are reminiscent of general issues in statistical analysis, with effect size and variability determining statistical significance. It may be worthwhile to consider testing several concentrations of a test compound rather than a single one in order to optimize analysis, which would additionally reveal aspects of concentration-response and of potency of the compound tested concentration [235].

We applied the commonly used LOOCV method to determine the robustness of our gene set as well its prediction. This validation showed that most exposures, when their data were not included in assembling the gene set, got comparable $p$-values based on the data of the remaining exposures. This indicates that the predictions were sufficiently robust against overfitting on compound-specific effects. Because not all genes met the $5 \%$ FDR threshold in all of the exposures, we were able to reduce the 132 genes in the Pennings_Diff_Correlation set to a smaller set of 52 genes. This latter set (Pennings_Diff_Crossval) also showed improved agreement with differentiation inhibition ( $R=-0.846$ ) as well as compound class (AUC $=0.889$ ) compared to previously described sets. Although its R and AUC values were not as high as the Pennings_Diff_Correlation set, it needs to be emphasized that larger set was obtained using the complete data set and therefore, on statistical grounds, some degree of overfitting can be expected. Therefore, because of the higher confidence restrictions applied, it is to be expected 
that the cross-validated gene set will probably give more realistic classification accuracies for applications in future studies.

In the comparisons of the classifications with the in vivo end point shown in Figure 2, it becomes clear that both Pennings_Diff sets perform at a comparable level as the standard EST end point, which has an AUC of 0.911. Although the EST end point of microscopically scoring of contracting cardiomyocyte foci is a useful and convenient end point, it has two main disadvantages. Not only is optical scoring somewhat subjective but, additionally, the EST works as a 'black box' that does not give any information on the mode of action of a compound. Transcriptomics and other molecular approaches [177, 256,262 ] can help to improve these aspects of the EST, in providing more quantitatively reliable data that can also be analyzed to determine which processes are affected by toxicant exposures. Such data can help construct a better informed picture of its applicability domain.

Our cross-validated set, as well as the initial set, contains a large number of genes involved in development or stem cell-related processes. Moreover, these genes show expression peaks occurring across the time range studied, and nearly half of the genes have some kind of mutual interaction. This finding illustrates the biological robustness of our set(s) and gives confidence on its application in future studies. Remarkably, in spite of the biological relevance of our set, it shows little overlap with previously published gene sets, with three genes (Frzb, Gas1, Mesp1) being included in the Van_Dartel_Heartdiff_24h set and none in the EST_biomarker_genes set. Partially, this can be attributed to the fact that these other gene sets were based on other (smaller) data sets, but moreover, these sets were both obtained as an answer to slightly different kinds of research questions. The Van_Dartel_Heartdiff_24h set was determined as genes upregulated at $24 \mathrm{~h}$ during normal ESC differentiation, whereas the EST_biomarker_genes set contains genes up- or down-regulated during 0 - 48h of normal ESC differentiation and also (up- or down-) regulated by five developmentally toxic compounds.

The varying toxicological responses found in the data indicate that several different mechanisms are involved for the compounds examined and that a method based on multiple genes covering multiple aspects of development - such as the developmental track - will allow optimal prediction of developmental toxicity. Interestingly, there are a number of genes for which some of the compounds examined gave comparable gene expression responses (Figure 4). Most notable here are the similar responses for the three triazoles studied (i.e. hexaconazole, triadimefon, flusilazole). Interestingly, these profiles are similar to that of retinoic acid, which lend further support to the hypothesis that their teratogenic mechanism lies in inhibiting common enzymes involved in retinoic acid metabolism, causing disrupted retinoic acid homeostasis [249]. Characteristic for these exposures were not only several homeobox containing genes, but also Arg1, Id2, and zinc finger protein 503 (Zfp503). This latter gene was previously identified as a puta- 
tive novel gene important for cardiac development [258], which is corroborated by its inclusion in the Pennings_Diff_Correlation set. Also, Chang et al. [263] described induction of this gene in embryonal carcinoma cells upon retinoic acid exposure, indicating a role in retinoic acid-induced developmental toxicity. A second example of a similar compound response is that of warfarin, methotrexate, and methyl mercury. In this case, the genes most affected include the known heart muscle protein Myl7 as well as Rbm24, and Cxcr4. These other two genes have more recently been found also to be involved in muscle development $[264,265]$. These findings indicate that besides more accurate or more quantitative developmental toxicity classification, our gene set (and therefore the 'differentiation track' method) can also serve to deduce mechanistic information from the data and possibly predict compound mode of actions using this method. Before such an approach can be effective, more compound and mechanistic data needs to be collected in a database suitable for compound comparison [238, 266]. Such additional compound data will in turn also allow for further improvement of gene sets as well as classification criteria for the 'differentiation track' algorithm.

In conclusion: by integrating the currently available data, we have identified a novel set of biologically relevant genes that allows for improved prediction of developmental toxicity. Using cross-validation, we confirmed that predictions using this set correlate better with the EST quantitative end point. Our data suggest that when combined with improvement of sample variability and effect size, predictions of close to $100 \%$ accuracy might be reached. We expect that our gene set can be of use in future studies aimed at improving the EST for risk assessment, thus making a next step towards regulatory implementation.

\section{Acknowledgments}

This study was supported by grant MFA6809 from the Dutch technology society foundation STW and by grant nr 050-06-510 from the Netherlands Genomics Initiative/Netherlands Organization for Scientific Research (NWO). 

Jeroen L.A. Pennings, Dorien A.M. van Dartel, Tessa E. Pronk, Peter J.M. Hendriksen, Aldert H. Piersma

Stem Cells and Development (2011) 20:115-126 
CHAPTER 10

Identification by gene co-regulation mapping of novel genes involved in embryonic stem cell differentiation 


\begin{abstract}
A combined analysis of data from a series of literature studies can lead to more reliable results than that based on a single study. A common problem in performing combined analyses of literature microarray gene expression data is that the original raw data are not always available and not always easy to combine in one analysis. We propose an approach that does not require analyzing original raw data, but instead takes literature gene sets derived from (supplementary) tables as input and uses gene co-occurrence in these sets for mapping a co-regulation network. An algorithm for this method was applied to a collection of literature derived gene sets related to embryonic stem cell differentiation. In the resulting network, genes involved in similar biological processes or expressed at similar time points during differentiation were found to cluster together. Using this information, we identified 43 genes not previously associated with cardiac embryonic stem cell differentiation for which we were able to assign a putative novel biological function. For six of these genes (Apobec2, Cth, Ptges, Rrad, Zfp57, and 2410146L05Rik), literature data on mouse knockout phenotypes supports their putative function. Three other genes (Rcor2, Zfp503, and Hspb3) are part of major pathways within the network and therefore likely mechanistically relevant candidate genes. We anticipate that these 43 genes can help to improve the understanding of the molecular events underlying embryonic stem cell differentiation. Moreover, the approach introduced here can be more widely applied to identify possible novel gene functions in biological processes.
\end{abstract}




\section{Introduction}

Combined analysis of microarray gene expression data from multiple studies offers the possibility to derive more reliable results than can be obtained from a single study. Although such combined analyses of microarray data are still comparatively rare, they have been successfully applied in well-studied research areas like cancer [267-269], host response to pathogens [270, 271], and aging [272]. However, for many microarray studies published in the literature, their inclusion in a combined analysis is hampered by the fact that their full (raw or normalized) gene expression data are not publicly accessible. Even if this is the case, differences in study designs or experimental procedures between studies can provide a practical barrier for inclusion. Furthermore, processing large amounts of data can be time-consuming and troublesome, strengthening the need for a convenient alternative.

We propose to use published microarray data in a different way, omitting normalization and statistical issues between different studies. We do this by looking for jointly regulated genes based on co-occurrence in individual experimental gene sets. A gene set is here defined as a list of genes that are described as meeting the same regulation criteria in a given literature study, such as being significantly up-regulated, down-regulated, or being clustered together based on having similar expression patterns. In fact, most microarray study publications provide (supplementary) tables with gene lists that meet criteria suitable for the underlying study. In many cases, a gene set will correspond directly to such a table, sometimes to table subsections that have different headers. If two genes occur together in a gene set in one publication, there is a reasonable chance that they are relevant to the process and also that they are to a certain extent co-regulated. If this is reported in multiple studies, both these chances become increasingly likely. Therefore, by analyzing a sufficiently large number of gene sets, it becomes possible to determine with high confidence which genes are commonly regulated and additionally share co-regulation. The latter information can be used to construct a co-regulation network, which can provide additional insights into the regulatory processes involved $[273,274]$. More specifically, if a gene with a hitherto unknown function is co-regulated with several genes that all are known to be involved in the same biological process, this gene then can be assumed to play a role in that process [275-277]. Furthermore, the number of connections (edges) that a gene has in a network can serve as an indicator for its importance in the network process [278].

In this study, we will show the applicability of our method using three different gene set collections. One of these collections, containing gene sets derived from studies on embryonic stem cell (ESC) differentiation, will be analyzed in more detail with a view to cardiomyocyte differentiation and its application in toxicity testing. 
During embryonic development, the heart is one of the first tissues to be formed and to acquire functionality. The cellular and molecular events involved in early cardiac development are highly conserved between different species (see review in $[279,280]$ ). Cardiac progenitor cells originate in the mesodermal germ layer and ultimately differentiate to cardiomyocytes via a cascade of development steps. Although molecular analysis of cardiac development has already identified several genes involved in (part of) this complex differentiation process, much is still unknown. Embryonic stem cell models are often used as a practical model system to study in vitro cardiomyocyte differentiation (reviewed in [281]). Several gene expression studies using this model system have been published, which allows for data from several related studies to be analyzed in combination.

We applied our method to construct and analyze a co-regulation network of ESC differentiation, and used this network to derive groups of co-regulated and functionally related genes. Using this approach, we identified several novel genes involved in the cardiac cell differentiation process.

\section{Methods}

\section{Libraries}

The first gene set collection used is the c2.cgp collection, which was downloaded from the MSigDB website (www.broadinstitute.org/gsea/msigdb/). This collection contains 1186 expert curated gene sets and a total number of 15891 unique gene identifiers (UGIs) derived from microarray experiments using chemical and genetic perturbations [82] and is to our knowledge the largest gene set collection publicly available. The second gene set collection is the lung-inflammation collection, derived from 12 microarray studies used in a meta-analysis on acute lung inflammation models from 45 exposures to air pollutants; bacterial, viral, and parasitic infections; and allergic asthma models [271]. This collection contains 90 gene sets and 2680 UGls.

We previously described the third gene set collection, which is the ESC-diff collection $[111,177]$ (and references sited therein). This collection contains gene sets derived from 42 microarray studies describing gene expression changes during the differentiation of ESC to several types of tissue, with a certain emphasis on cardiomyocytes. During the collecting process, gene sets were included if the underlying study was considered relevant based on mutual agreement by a developmental toxicologist and bioinformatician. To avoid redundancy, gene sets obtained using the Anni textmining tool were excluded for the present study as textmining is implicitly using literature data. The resulting gene set collection used contained 167 gene sets and a total number of 15196 UGIs. 
Co-regulation mapping

Gene co-regulation mapping was determined using an algorithm written in the statistical software environment R (www.r-project.org). Starting from a gene set collection in tab-delimited file format, UGls were converted to uppercase (for computational convenience) and subsequently non-meaningful UGls like "0" or "-" were removed. For the resulting gene symbols, the number of occurrences in the collection was calculated. For each combination of two (non-identical) symbols, co-regulation was determined using two criteria. First, the absolute number of sets in which both genes co-occurred had to be at least a certain (user-definable) threshold, to select for often co-regulated genes and ensure biological relevance. Second, relative co-regulation strength, calculated as the cosine correlation of gene set co-occurrences, had to be at least $50 \%$. This is calculated as $N_{a, b} / \sqrt{ }\left(N_{a}{ }^{*} N_{b}\right)$, where $N_{a, b}$ is the number of sets in which gene $A$ and geneB occur together, and $\mathrm{N}_{\mathrm{a}}$ and $\mathrm{N}_{\mathrm{b}}$ are the number of occurrences of respectively gene $\mathrm{A}$ and geneB in all sets. If both these criteria were met, the two gene symbols were connected in a network description file for visualization with Cytoscape [127]. The criteria for choosing the userdefinable threshold were tested and optimized using the c2.cgp and lung-inflammation collection. For further analyses on these and the ESC-diff collection, a default stringency was defined so that the threshold for each data set led to approximately $5 \%$ of the total number of UGls becoming mapped in the network description file.

The resulting co-regulation network was further visualized using Gene Ontology functional annotations, or gene expression data from earlier studies [177, 271]. The presence of significantly densely connected clusters within the total network was determined using the Cytoscape plugin MCODE [282] using default settings.

Identification of novel genes involved in embryonic stem cell cardiac differentiation

For the genes in the ESC-diff co-regulation network mapping, Gene Ontology functional annotation and enrichment was determined using the Cytoscape plugin BiNGO [283] in combination with the DAVID web application (david.abcc.ncifcrf.gov) [183]. Genes were considered already associated with ESC differentiation if they were assigned to one of the following Gene Ontology terms: development processes (including stem cell, organ, heart, or muscle development), cell differentiation, cell proliferation, extracellular matrix (including collagen), contractile fiber, muscle system process (including muscle contraction), and heart contraction. Furthermore, genes for which the textmining tool Anni [138] found concept weights $>10^{-5}$ for the above mentioned terms or the concepts "stem cells", "gastrulation" or "ectoderm/endoderm/mesoderm formation" were considered not novel for stem cell differentiation. The concept weight is a measure Anni uses to indicate the degree of literature association of one term with another. We found that when distributions for a larger number of concept weights are combined, the distribution approaches its background level at concept weights around $10^{-5}$, and therefore concept 
weights below this value are considered as not indicative for literature association. For the genes not functionally annotated by Gene Ontology or Anni, those that were part of an MCODE cluster enriched for one of the above functions were considered candidates for such a function.

\section{Results}

Method development

To show the applicability of our approach and to optimize the criteria used, we applied our algorithm to two gene set collections that are different in nature. The first of these is the MSigDB c2.cgp collection [82], which contains 1186 gene sets related to a wide range of chemical and genetic perturbations. Second, the lung-inflammation collection, with 90 gene sets, which is smaller and more focused on acute lung inflammation models. For both gene set collections we found that biologically meaningful co-regulation networks could be obtained (Fig 1A, 1B). For the c2.cgp collection, various co-regulation networks of varying sizes were found. Several of these consisted of genes with a common related functionality such as cell division or immunological response (Fig 1A). For the lung-inflammation collection, besides some smaller networks, a large and dense network of commonly inflammation-induced genes containing 120 genes was found (Fig 1B). This network includes 66 immune response genes, 61 of which overlap with the 100 immune response genes found previously as part of a full-scale meta-analysis [5]. Moreover, this network contains all of the 23 'core inflammation response' genes found in the previous meta-analysis.

Embryonic stem cell differentiation mapping

When the algorithm was applied to the ESC-diff gene set collection a large and densely interconnected network with 927 genes and 7402 edges was obtained (Fig 1C). The network appeared to have two major sub-networks, at the left and right hand side, respectively. This finding was found to be robust for various stringency settings, as was the observation that both major sub-networks comprised two minor sub-networks, The overall network was significantly enriched for several GO terms related to development, differentiation and muscle function. In addition, enrichment for several other functional categories was found, especially transcription factors, cell proliferation, small heat shock proteins, and several types of metabolite transport. However, we found that known cardiomyocyte marker genes were located exclusively in the lower right hand side of the network, whereas stem cell pluripotency markers were located only in the top left. This suggests that these two areas correspond to different processes or phases in stem cell differentiation. Both the functional and timing aspects were examined further. 


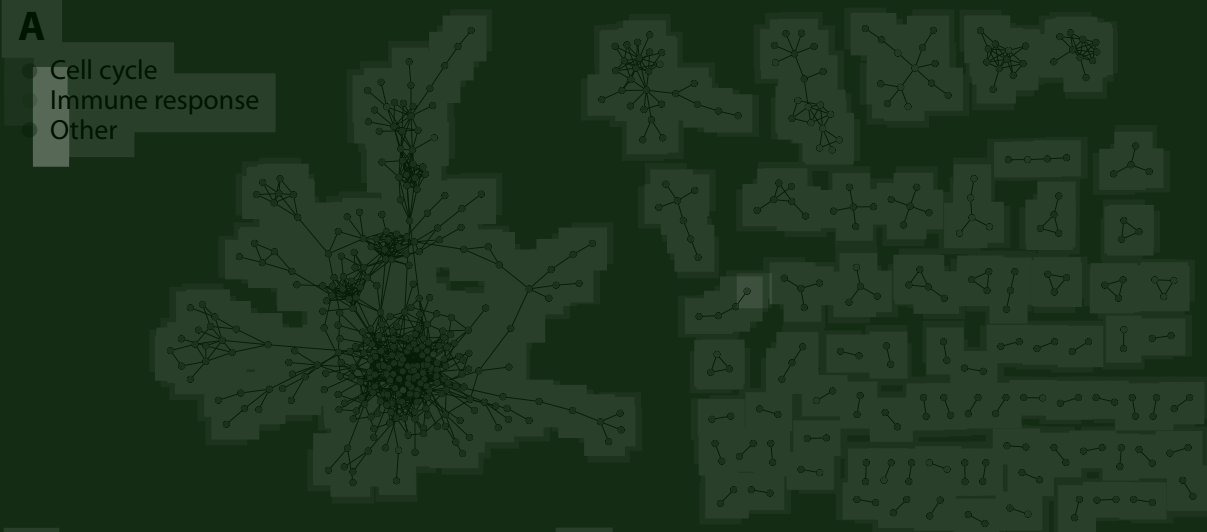

B

- Core inflammation response

Other immune response

- Other

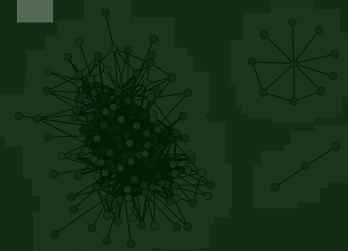

C

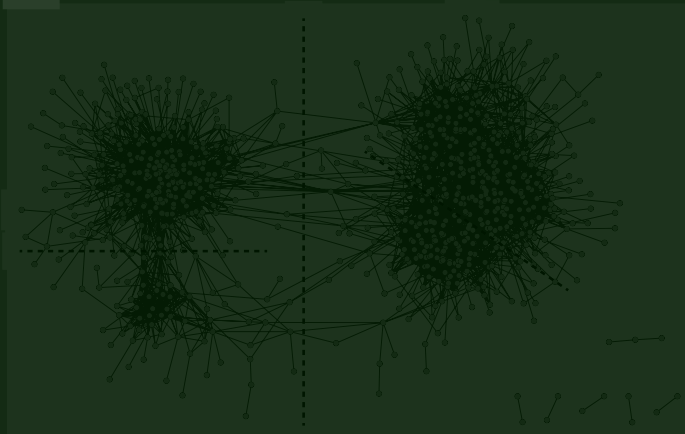

Figure 1. Network visualizations for three gene set collections. A, network for c2.cgp collection (591 genes, 1243 edges); B, network for lung-inflammation collection (133 genes, 930 edges); C, network for ESC-diff (embryonic stem cell differentiation) collection (927 genes, 7402 edges) with transitions between sub-networks indicated as dashed lines. For color figure, see Color figures section.

By using the MCODE plugin, we identified 21 clusters within the overall network that are significantly densely connected. The size of these clusters varied from 4 to 39 genes, with an overall total of 304 genes. Several of these clusters were enriched for specific GO-terms. Clusters with such similar GO-term enrichments appeared together in the network graph and (by making small adjustments to the MCODE cluster size threshold parameter) it was found that they were mutually strongly connected. Taken together, three clusters groups could be distinguished (Fig 2A, Table 1,2,3, supplementary Table 1). The first group contained five clusters ( 87 genes, upper left side of Fig $2 A$ ) that were mainly enriched for early developmental GO-terms such as stem cell development, embryonic development, and regulation of cell proliferation. The second group, containing three clusters ( 25 genes, upper right side of Fig $2 \mathrm{~A}$ ), was enriched for terms such as organ development and embryonic development and GO-terms involved in later development stages. The third group (55 genes, lower right side of Fig 2A) contained three clusters and was enriched for specific heart- and muscle-related GO-terms such as 
muscle development, muscle contraction, and heart development. In addition to development- and muscle-related processes, the first two groups were enriched for transcription factors, and the third group for small heat shock proteins. Next to the clusters in the three mentioned groups, five clusters (61 genes) were enriched for other biological processes related to transport and/or metabolism, and five other clusters ( 76 genes) did not show functional enrichment. The three groups mentioned above are each found in a distinct minor sub-network. The fourth minor sub-network in the lower left side of Figure $2 \mathrm{~A}$ contains several genes that can be associated with early development, proliferation, pluripotency, as well as several metabolic processes. However, the two clusters in this sub-network do not reach statistically significant functional enrichment.

A

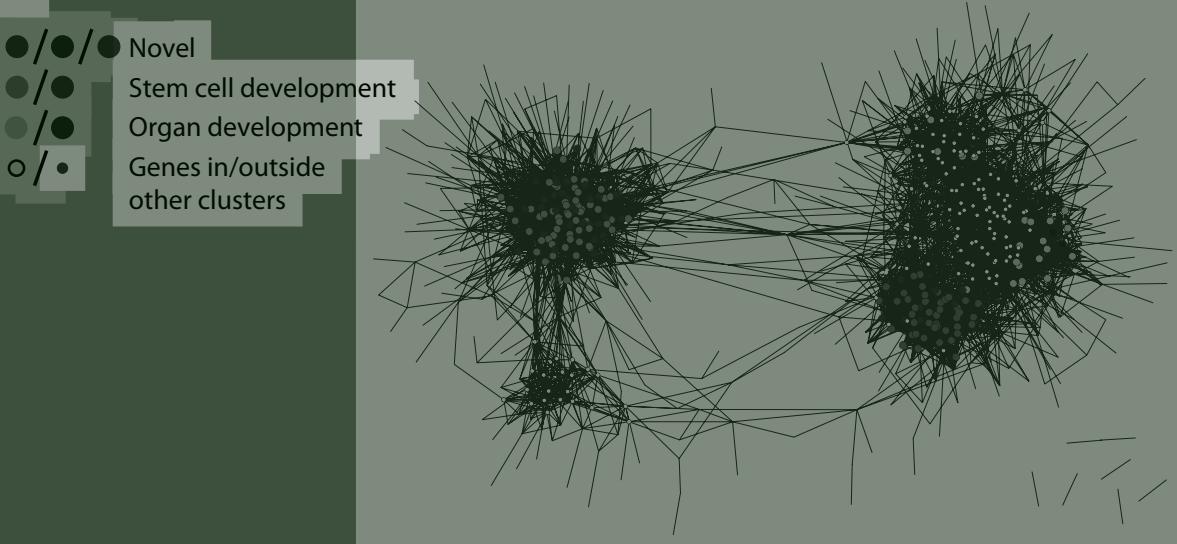

B
Early (day 3)
Middle (day 4-5)
Late (day 6-7)
Early and late
O No data

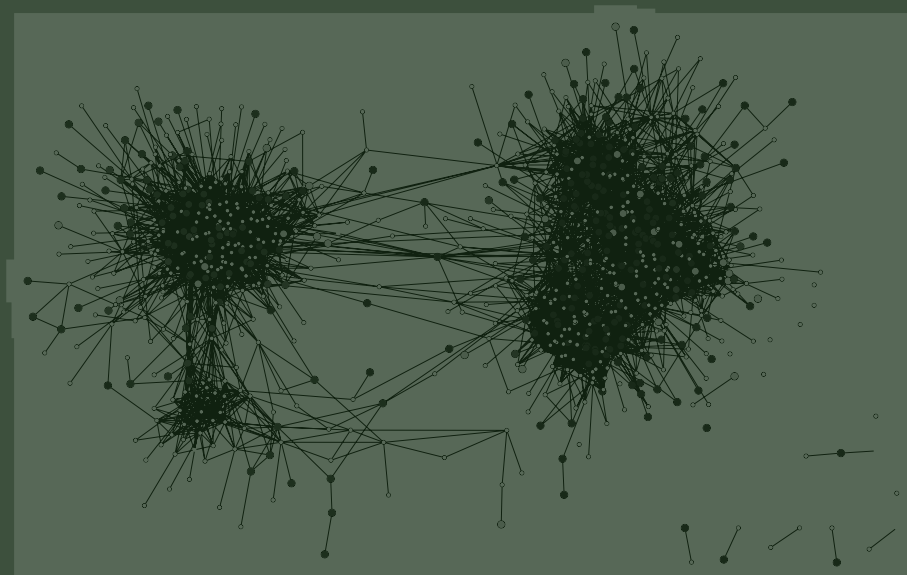

Figure 2. Embryonic stem cell cardiomyocyte differentiation co-regulation network. A, biological process enrichment in network clusters containing novel functional candidates; $\mathrm{B}$, peak gene expression for genes in a stem cell differentiation time series. For color figure, see Color figures section. 
Table 1. Identified genes in cluster group 1 (associated with stem cell development, embryonic development, regulation of cell proliferation).

\begin{tabular}{|c|c|c|c|c|}
\hline Gene symbol & Gene description & Novel & $\begin{array}{l}\text { MCODE } \\
\text { cluster }\end{array}$ & Edges \\
\hline 1700019D03RIK & RIKEN cDNA 1700019D03 gene & yes & 2 & 74 \\
\hline 2310003C23RIK & RIKEN cDNA $2310003 C 23$ gene & yes & 2 & 49 \\
\hline AVPI1 & arginine vasopressin-induced 1 & yes & 2 & 36 \\
\hline LRRC2 & leucine rich repeat containing 2 & yes & 2 & 69 \\
\hline PIPOX & pipecolic acid oxidase & yes & 2 & 48 \\
\hline RCOR2 & rest corepressor 2 & yes & 2 & 59 \\
\hline SLC25A36 & solute carrier family 25, member 36 & yes & 2 & 31 \\
\hline STOML1 & stomatin-like 1 & yes & 2 & 28 \\
\hline TMEM8 & $\begin{array}{l}\text { transmembrane protein } 8 \text { (five membrane-spanning } \\
\text { domains) }\end{array}$ & yes & 2 & 42 \\
\hline UPP1 & uridine phosphorylase 1 & yes & 2 & 51 \\
\hline ZFP57 & zinc finger protein 57 & yes & 2 & 51 \\
\hline COBL & cordon-bleu & no & 2 & 52 \\
\hline ETV5 & ets variant gene 5 & no & 2 & 85 \\
\hline JARID2 & jumonji, AT rich interactive domain 2 & no & 2 & 83 \\
\hline KLF9 & Kruppel-like factor 9 & no & 2 & 41 \\
\hline LEFTY1 & left right determination factor 1 & no & 2 & 67 \\
\hline MYCN & $\mathrm{N}$-myc proto-oncogene & no & 2 & 68 \\
\hline NROB1 & nuclear receptor subfamily 0 , group $B$, member 1 & no & 2 & 69 \\
\hline NTN1 & netrin 1 & no & 2 & 47 \\
\hline PHC1 & polyhomeotic-like 1 (Drosophila) & no & 2 & 62 \\
\hline REST & RE1-silencing transcription factor & no & 2 & 40 \\
\hline RIF1 & Rap1 interacting factor 1 homolog (yeast) & no & 2 & 88 \\
\hline SPRY2 & sprouty homolog 2 (Drosophila) & no & 2 & 65 \\
\hline SPRY4 & sprouty homolog 4 (Drosophila) & no & 2 & 79 \\
\hline TCFCP2L1 & transcription factor $\mathrm{CP} 2$-like 1 & no & 2 & 67 \\
\hline ZIC5 & zinc finger protein of the cerebellum 5 & no & 2 & 52 \\
\hline 2200001115RIK & RIKEN cDNA 2200001115 gene & yes & 6 & 30 \\
\hline IFITM2 & interferon induced transmembrane protein 2 & yes & 6 & 43 \\
\hline JAM2 & junction adhesion molecule 2 & yes & 6 & 75 \\
\hline MANBA & mannosidase, beta a, lysosomal & yes & 6 & 61 \\
\hline MKRN1 & makorin, ring finger protein, 1 & yes & 6 & 59 \\
\hline PDCL2 & phosducin-like 2 & yes & 6 & 25 \\
\hline $\mathrm{TDH}$ & L-threonine dehydrogenase & yes & 6 & 69 \\
\hline CTNNAL1 & catenin (cadherin associated protein), alpha-like 1 & no & 6 & 23 \\
\hline DPPA3 & developmental pluripotency-associated 3 & no & 6 & 65 \\
\hline ENAH & enabled homolog (Drosophila) & no & 6 & 50 \\
\hline
\end{tabular}




\begin{tabular}{|c|c|c|c|c|}
\hline Gene symbol & Gene description & Novel & $\begin{array}{l}\text { MCODE } \\
\text { cluster }\end{array}$ & Edges \\
\hline GBX2 & gastrulation brain homeobox 2 & no & 6 & 43 \\
\hline GPA33 & glycoprotein A33 (transmembrane) & no & 6 & 35 \\
\hline GRSF1 & G-rich RNA sequence binding factor 1 & no & 6 & 23 \\
\hline IFITM1 & interferon induced transmembrane protein 1 & no & 6 & 29 \\
\hline KLF2 & Kruppel-like factor 2 (lung) & no & 6 & 40 \\
\hline LEFTY2 & left-right determination factor 2 & no & 6 & 33 \\
\hline MORC1 & microrchidia 1 & no & 6 & 50 \\
\hline NANOG & Nanog homeobox & no & 6 & 29 \\
\hline OTX2 & orthodenticle homeobox 2 & no & 6 & 60 \\
\hline PCAF & p300/CBP-associated factor & no & 6 & 33 \\
\hline SALL1 & sal-like 1 (Drosophila) & no & 6 & 50 \\
\hline SERTAD2 & SERTA domain containing 2 & no & 6 & 50 \\
\hline TGIF & TGFB-induced factor homeobox & no & 6 & 28 \\
\hline TLE4 & $\begin{array}{l}\text { transducin-like enhancer of split } 4 \text {, homolog of Drosophila } \\
\text { E(spl) }\end{array}$ & no & 6 & 51 \\
\hline ZFP64 & zinc finger protein 64 & no & 6 & 24 \\
\hline ZIC3 & zinc finger protein of the cerebellum 3 & no & 6 & 35 \\
\hline 2410146L05RIK & RIKEN cDNA 2410146L05 gene & yes & 17 & 29 \\
\hline ACP6 & lysophosphatidic acid phosphatase & yes & 17 & 35 \\
\hline CDYL2 & chromodomain protein, Y chromosome-like 2 & yes & 17 & 16 \\
\hline EPB4.1L4A & erythrocyte protein band 4.1-like 4a & yes & 17 & 17 \\
\hline PTGES & prostaglandin E synthase & yes & 17 & 24 \\
\hline FGF4 & fibroblast growth factor 4 & no & 17 & 35 \\
\hline $\mathrm{HCK}$ & hemopoietic cell kinase & no & 17 & 21 \\
\hline HK2 & hexokinase 2 & no & 17 & 20 \\
\hline IGF2BP1 & insulin-like growth factor 2 mRNA binding protein 1 & no & 17 & 36 \\
\hline MRAS & muscle and microspikes RAS & no & 17 & 19 \\
\hline $\mathrm{NCL}$ & nucleolin & no & 17 & 18 \\
\hline NPHS1 & nephrosis 1 homolog, nephrin (human) & no & 17 & 19 \\
\hline RARG & retinoic acid receptor, gamma & no & 17 & 29 \\
\hline SGK & serum/glucocorticoid regulated kinase & no & 17 & 45 \\
\hline TCFAP2C & transcription factor AP-2, gamma & no & 17 & 34 \\
\hline $\mathrm{CTH}$ & cystathionase (cystathionine gamma-lyase) & yes & 18 & 47 \\
\hline D14ERTD436E & DNA segment, chr 14 , erato doi 436 , expressed & yes & 18 & 19 \\
\hline NFATC2IP & $\begin{array}{l}\text { NFAT, cytoplasmic, calcineurin-dependent } 2 \text { interacting } \\
\text { protein }\end{array}$ & yes & 18 & 33 \\
\hline RANBP17 & RAN binding protein 17 & yes & 18 & 17 \\
\hline SNX10 & sorting nexin 10 & yes & 18 & 25 \\
\hline FOXD3 & forkhead box D3 & no & 18 & 49 \\
\hline
\end{tabular}




\begin{tabular}{lllll}
\hline Gene symbol & Gene description & Novel & $\begin{array}{l}\text { MCODE } \\
\text { cluster }\end{array}$ & Edges \\
\hline POU5F1 & POU domain, class 5, transcription factor 1 & no & 18 & 32 \\
SIX4 & sine oculis-related homeobox 4 homolog (Drosophila) & no & 18 & 17 \\
SOCS2 & suppressor of cytokine signaling 2 & no & 18 & 43 \\
SUZ12 & suppressor of zeste 12 homolog (Drosophila) & no & 18 & 14 \\
TDGF1 & teratocarcinoma-derived growth factor 1 & no & 18 & 41 \\
ZFP36L1 & zinc finger protein 36, C3H type-like 1 & no & 18 & 46 \\
AGTRAP & angiotensin II, type I receptor-associated protein & yes & 19 & 22 \\
ASS1 & argininosuccinate synthetase 1 & yes & 19 & 11 \\
DPPA5 & developmental pluripotency associated 5 & no & 19 & 33 \\
ESRRB & estrogen related receptor, beta & no & 19 & 42 \\
KLF4 & Kruppel-like factor 4 (gut) & no & 19 & 32 \\
MYBL2 & myeloblastosis oncogene-like 2 & no & 19 & 46 \\
TCL1 & T-cell lymphoma breakpoint 1 & no & 19 & 29 \\
TRP53 & transformation related protein 53 & no & 19 & 15 \\
\hline
\end{tabular}

To visualize the differentiation process in an alternative manner, we used data from a recent study in which we described gene expression changes during embryonic stem cell cardiac muscle cell differentiation [177]. This study was not included in the ESC-diff gene set collection and therefore provided independent data. For the genes for which differential expression was found, we used different colors to indicate at which differentiation phase these genes had their highest expression. This visualization indicated that the left and right side of the network can be associated with early (undifferentiated stem cells) and late (more differentiated) time points, respectively (Fig 2B). This is in agreement with the different functionalities based on GO-term enrichment. Genes expressed during intermediate time points did not group together, but appeared in either of the two network sides.

When these two visualization approaches were combined again, we found that out of the 167 genes in the three cluster groups (Figure 2A, Table 1,2,3), independent data from the van Dartel [177] study showed differential expression for 49 of these genes (29\%, compared to $6 \%$ for the whole-genome data). Of these, 18 early expressed genes were all found in the stem cell development group (group 1). Of the 4 mid-phase expressed genes, 2 were found in the stem cell development group and 2 in the organ development group (group 2). Of the 26 late expressed genes, 2 were found in the stem cell differentiation group, 4 in the organ development group, and 20 in the muscle/heart function group (group 3). Finally, one gene with both early and late high expression was found in the stem cell development group. 
Table 2. Identified genes in cluster group 2 (associated with organ development, embryonic development).

\begin{tabular}{|c|c|c|c|c|}
\hline Gene symbol & Gene description & Novel & $\begin{array}{l}\text { MCODE } \\
\text { cluster }\end{array}$ & Edges \\
\hline COLEC12 & collectin sub-family member 12 & yes & 9 & 8 \\
\hline ZFP503 & zinc finger protein 503 & yes & 9 & 14 \\
\hline FOXC2 & forkhead box C2 & no & 9 & 10 \\
\hline GATA5 & GATA binding protein 5 & no & 9 & 7 \\
\hline HEY1 & hairy/enhancer-of-split related with YRPW motif 1 & no & 9 & 33 \\
\hline HOXA3 & homeo box $\mathrm{A} 3$ & no & 9 & 17 \\
\hline HOXB2 & homeo box B2 & no & 9 & 42 \\
\hline HOXB4 & homeo box B4 & no & 9 & 15 \\
\hline PDGFRA & platelet derived growth factor receptor, alpha polypeptide & no & 9 & 29 \\
\hline RYR2 & ryanodine receptor 2 , cardiac & no & 9 & 10 \\
\hline SPON1 & spondin 1, (f-spondin) extracellular matrix protein & no & 9 & 60 \\
\hline TBX2 & T-box 2 & no & 9 & 28 \\
\hline ZFPM2 & zinc finger protein, multitype 2 & no & 9 & 37 \\
\hline COL2A1 & collagen, type II, alpha 1 & no & 12 & 20 \\
\hline FLRT2 & fibronectin leucine rich transmembrane protein 2 & no & 12 & 21 \\
\hline ISL1 & ISL1 transcription factor, LIM/homeodomain & no & 12 & 25 \\
\hline MEIS1 & Meis homeobox 1 & no & 12 & 21 \\
\hline MRG1 & Meis1-related gene 1 & no & 12 & 13 \\
\hline GAS6 & growth arrest specific 6 & no & 14 & 18 \\
\hline HDAC7A & histone deacetylase 7A & no & 14 & 14 \\
\hline ITGB1 & integrin beta 1 (fibronectin receptor beta) & no & 14 & 18 \\
\hline MMP14 & matrix metallopeptidase 14 (membrane-inserted) & no & 14 & 15 \\
\hline PEG3 & paternally expressed 3 & no & 14 & 18 \\
\hline TIMP2 & tissue inhibitor of metalloproteinase 2 & no & 14 & 17 \\
\hline VCL & vinculin & no & 14 & 14 \\
\hline
\end{tabular}

Novel embryonic stem cell cardiac differentiation genes

Using Gene Ontology for functional annotation of the genes in the embryonic stem cell development network, we found that of the 927 genes, 521 could be associated with GO processes relevant for ESC differentiation, and 418 with related Anni textmining concepts. Leaving out these genes left 327 genes not yet described to be involved in ESC differentiation, 165 and 162 of which were located at the left and right hand side of the network, respectively. Of these novel genes, 43 were also part of a significantly dense MCODE cluster with a functional enrichment for a GO-term related to ESC or cardiac differentiation, making them candidate genes for having a corresponding function assigned to them. These 43 genes are listed in Table 4 and indicated (in dark red, dark green or dark blue) in Figure 2. Concerning the overlap with other pathways found 
Table 3. Identified genes in cluster group 3 (associated with muscle development, muscle contraction, heart development).

\begin{tabular}{|c|c|c|c|c|}
\hline Gene symbol & Gene description & Novel & $\begin{array}{l}\text { MCODE } \\
\text { cluster }\end{array}$ & Edges \\
\hline APOBEC2 & apolipoprotein B editing complex 2 & yes & 1 & 57 \\
\hline G0S2 & G0/G1 switch gene 2 & yes & 1 & 40 \\
\hline HSPB3 & heat shock protein 3 & yes & 1 & 39 \\
\hline PPP1R14C & $\begin{array}{l}\text { protein phosphatase 1, regulatory (inhibitor) subunit } \\
14 C\end{array}$ & yes & 1 & 46 \\
\hline RRAD & ras-related associated with diabetes & yes & 1 & 67 \\
\hline SYNPO2L & synaptopodin 2-like & yes & 1 & 72 \\
\hline ACTN2 & actinin alpha 2 & no & 1 & 77 \\
\hline ASB2 & ankyrin repeat and SOCS box-containing 2 & no & 1 & 51 \\
\hline ATP2A2 & $\begin{array}{l}\text { ATPase, Ca++ transporting, cardiac muscle, slow twitch } \\
2\end{array}$ & no & 1 & 43 \\
\hline CKM & creatine kinase, muscle & no & 1 & 57 \\
\hline CSRP3 & cysteine and glycine-rich protein 3 & no & 1 & 74 \\
\hline ENO3 & enolase 3 , beta muscle & no & 1 & 55 \\
\hline HSPB2 & heat shock protein 2 & no & 1 & 69 \\
\hline HSPB7 & heat shock protein family, member 7 (cardiovascular) & no & 1 & 62 \\
\hline ITGB1BP2 & integrin beta 1 binding protein 2 & no & 1 & 50 \\
\hline LAMA4 & laminin, alpha 4 & no & 1 & 48 \\
\hline LDB3 & LIM domain binding 3 & no & 1 & 58 \\
\hline MB & myoglobin & no & 1 & 44 \\
\hline MYBPC3 & myosin binding protein $C$, cardiac & no & 1 & 39 \\
\hline MYH6 & myosin, heavy polypeptide 6 , cardiac muscle, alpha & no & 1 & 78 \\
\hline MYL3 & myosin, light polypeptide 3 & no & 1 & 60 \\
\hline MYL4 & myosin, light polypeptide 4 & no & 1 & 55 \\
\hline MYL7 & myosin, light polypeptide 7 , regulatory & no & 1 & 52 \\
\hline MYOM1 & myomesin 1 & no & 1 & 34 \\
\hline MYOZ2 & myozenin 2 & no & 1 & 70 \\
\hline NPPA & natriuretic peptide precursor type $A$ & no & 1 & 55 \\
\hline PGAM2 & phosphoglycerate mutase 2 & no & 1 & 45 \\
\hline PLN & phospholamban & no & 1 & 71 \\
\hline POPDC2 & popeye domain containing 2 & no & 1 & 48 \\
\hline PPP1R12B & $\begin{array}{l}\text { protein phosphatase 1, regulatory (inhibitor) subunit } \\
12 B\end{array}$ & no & 1 & 33 \\
\hline SH3BGR & SH3-binding domain glutamic acid-rich protein & no & 1 & 58 \\
\hline SMPX & small muscle protein, $\mathrm{X}$-linked & no & 1 & 66 \\
\hline TCAP & titin-cap & no & 1 & 48 \\
\hline TNNC1 & troponin C, cardiac/slow skeletal & no & 1 & 60 \\
\hline TNNI1 & troponin I, skeletal, slow 1 & no & 1 & 52 \\
\hline
\end{tabular}




\begin{tabular}{|c|c|c|c|c|}
\hline Gene symbol & Gene description & Novel & $\begin{array}{l}\text { MCODE } \\
\text { cluster }\end{array}$ & Edges \\
\hline TNNI3 & troponin I, cardiac 3 & no & 1 & 59 \\
\hline TNNT2 & troponin $\mathrm{T} 2$, cardiac & no & 1 & 63 \\
\hline TRIM63 & tripartite motif-containing 63 & no & 1 & 32 \\
\hline TTN & titin & no & 1 & 55 \\
\hline 2310046A06RIK & RIKEN cDNA 2310046A06 gene & yes & 7 & 36 \\
\hline TM4SF1 & transmembrane 4 superfamily member 1 & yes & 7 & 45 \\
\hline ACTA1 & actin, alpha 1, skeletal muscle & no & 7 & 37 \\
\hline CRYAB & crystallin, alpha B & no & 7 & 42 \\
\hline DES & desmin & no & 7 & 45 \\
\hline DKK3 & dickkopf homolog 3 (Xenopus laevis) & no & 7 & 29 \\
\hline EEF1A2 & eukaryotic translation elongation factor 1 alpha 2 & no & 7 & 24 \\
\hline IL6 & interleukin 6 & no & 7 & 31 \\
\hline NPPB & natriuretic peptide precursor type B & no & 7 & 37 \\
\hline PTX3 & pentraxin 3 & no & 7 & 38 \\
\hline ALPK2 & alpha-kinase 2 & yes & 11 & 26 \\
\hline NEBL & nebulette & yes & 11 & 27 \\
\hline RCSD1 & RCSD domain containing 1 & yes & 11 & 31 \\
\hline MYL2 & myosin, light polypeptide 2 , regulatory, cardiac, slow & no & 11 & 29 \\
\hline MYOCD & myocardin & no & 11 & 26 \\
\hline SMYD1 & SET and MYND domain containing 1 & no & 11 & 21 \\
\hline
\end{tabular}

previously, among these novel genes there were no genes involved in cell proliferation, there were three transcription factors (Rcor2, Zfp57, Zfp503), and one heat shock protein (Hspb3).

\section{Discussion}

Since its introduction, microarray technology has grown to become used in over 6000 PubMed-publications per year. The growth in the number of studies published has been followed by the development and application of various methods for combined or meta-analysis (reviewed in [284]). However, there are several practical issues related to such analyses of gene expression data, and one major hurdle is the limited public availability of the complete raw or normalized data sets used in literature studies. Several approaches to combined or meta-analyses have been published in the last few years and although this field is still developing, it becomes apparent that no single best method exists as the suitability of an approach will depend on the nature and quality of available data. In this study we propose an approach which is not based on the actual re-analysis 
Table 4. Identified novel genes with functional evidence for a role in embryonic stem cell differentiation.

\begin{tabular}{|c|c|c|c|}
\hline Gene symbol & Gene description & $\begin{array}{l}\text { MCODE } \\
\text { cluster }\end{array}$ & Figure location \\
\hline 1700019D03RIK & RIKEN cDNA 1700019D03 gene & 2 & Upper left \\
\hline 2310003C23RIK & RIKEN cDNA 2310003 C23 gene & 2 & Upper left \\
\hline AVPI1 & arginine vasopressin-induced 1 & 2 & Upper left \\
\hline LRRC2 & leucine rich repeat containing 2 & 2 & Upper left \\
\hline PIPOX & pipecolic acid oxidase & 2 & Upper left \\
\hline RCOR2 & rest corepressor 2 & 2 & Upper left \\
\hline SLC25A36 & solute carrier family 25, member 36 & 2 & Upper left \\
\hline STOML1 & stomatin-like 1 & 2 & Upper left \\
\hline TMEM8 & $\begin{array}{l}\text { transmembrane protein } 8 \text { (five membrane-spanning } \\
\text { domains) }\end{array}$ & 2 & Upper left \\
\hline UPP1 & uridine phosphorylase 1 & 2 & Upper left \\
\hline ZFP57 & zinc finger protein 57 & 2 & Upper left \\
\hline $2200001115 R I K$ & RIKEN cDNA $2200001 / 15$ gene & 6 & Upper left \\
\hline IFITM2 & interferon induced transmembrane protein 2 & 6 & Upper left \\
\hline JAM2 & junction adhesion molecule 2 & 6 & Upper left \\
\hline MANBA & mannosidase, beta a, lysosomal & 6 & Upper left \\
\hline MKRN1 & makorin, ring finger protein, 1 & 6 & Upper left \\
\hline PDCL2 & phosducin-like 2 & 6 & Upper left \\
\hline $\mathrm{TDH}$ & L-threonine dehydrogenase & 6 & Upper left \\
\hline 2410146L05RIK & RIKEN cDNA 2410146L05 gene & 17 & Upper left \\
\hline ACP6 & lysophosphatidic acid phosphatase & 17 & Upper left \\
\hline CDYL2 & chromodomain protein, Y chromosome-like 2 & 17 & Upper left \\
\hline EPB4.1L4A & erythrocyte protein band 4.1-like $4 a$ & 17 & Upper left \\
\hline PTGES & prostaglandin E synthase & 17 & Upper left \\
\hline $\mathrm{CTH}$ & cystathionase (cystathionine gamma-lyase) & 18 & Upper left \\
\hline D14ERTD436E & DNA segment, chr 14 , erato doi 436 , expressed & 18 & Upper left \\
\hline NFATC2IP & $\begin{array}{l}\text { NFAT, cytoplasmic, calcineurin-dependent } 2 \text { interacting } \\
\text { protein }\end{array}$ & 18 & Upper left \\
\hline RANBP17 & RAN binding protein 17 & 18 & Upper left \\
\hline SNX10 & sorting nexin 10 & 18 & Upper left \\
\hline AGTRAP & angiotensin II, type I receptor-associated protein & 19 & Upper left \\
\hline ASS1 & argininosuccinate synthetase 1 & 19 & Upper left \\
\hline COLEC12 & collectin sub-family member 12 & 9 & Upper right \\
\hline ZFP503 & zinc finger protein 503 & 9 & Upper right \\
\hline APOBEC2 & apolipoprotein B editing complex 2 & 1 & Lower right \\
\hline G0S2 & G0/G1 switch gene 2 & 1 & Lower right \\
\hline HSPB3 & heat shock protein 3 & 1 & Lower right \\
\hline
\end{tabular}




\begin{tabular}{lllc}
\hline Gene symbol & Gene description & $\begin{array}{l}\text { MCODE } \\
\text { cluster }\end{array}$ & Figure location \\
\hline PPP1R14C & $\begin{array}{l}\text { protein phosphatase 1, regulatory (inhibitor) subunit } \\
\text { RRAD }\end{array}$ & 1 & Lower right \\
SYNPO2L & ras-related associated with diabetes & 1 & Lower right \\
2310046A06RIK & synaptopodin 2-like & 1 & Lower right \\
TM4SF1 & transmembrane 4 superfamily member 1 & 7 & Lower right \\
ALPK2 & alpha-kinase 2 & 7 & Lower right \\
NEBL & nebulette & 11 & Lower right \\
RCSD1 & RCSD domain containing 1 & 11 & Lower right \\
\hline
\end{tabular}

of original data, but on the co-occurrence of genes based on processing of gene sets derived from these data. One of the rationales behind our approach is the need for a combined analysis that allows inclusion of large numbers of literature microarray study results, even if the full original data are not (or not easily) available. Our algorithm for network construction allows the inclusion of almost any published study and furthermore does not require elaborate data processing or new software implementations; $R$ and Cytoscape are freely available and already familiar to most bioinformaticians. The algorithm used by us is available as supplementary data.

The three underlying concepts for our approach have each already been described on their own. Firstly, co-expression or co-regulation networks [274] have become a commonly applied method to infer novel gene functionality [273, 275-277]. Secondly, algorithms for determining gene co-occurrence in literature publications have been applied in textmining applications [285-288]. However, whereas textmining is mostly based on literature abstracts or database description fields, our approach specifically searches in (supplementary) tables, allowing the use of information that would otherwise have been overlooked. Thirdly, employing gene sets rather than full microarray data on the one hand or literature abstracts on the other has been described in several applications [126, 289-291]. These approaches, however, look for statistically significant overlap between lists, or to an experimental gene set supplied by the user. Therefore, the combination of these methods provides a novel integrated type of approach that should be able to incorporate some of the advantages of each of the underlying methods. A potential weakness of our approach is that the quality of the gene set collection taken as the starting point for this method will depend on the quality and relevance of the input data. We had to rely on the analyses done by the authors of the underlying studies, including the assessment of the reviewers and editors responsible for the process of peer review.

As part of the algorithm development, our approach was tested on two gene set collections, which were selected to be different in nature. Based on the multi-purpose c2.cgp collection, a large number of small networks were identified, including cell divi- 
sion and immunological response networks (Fig 1A). The co-regulation network for the lung-inflammation gene set collection found a large network, of mainly immunological genes (Fig 1B), which comprises the majority of the immunological genes that were found regulated by a previous full meta-analysis [271]. Moreover, it contains all of the 23 "core inflammation response" genes found previously [271]. These findings illustrate the usefulness of our approach.

For these two gene set collections, the examples given were obtained using stringency settings for which the number of genes in the network description file is around $5 \%$ of the number of unique gene identifiers in the collection, and these settings were chosen as the default stringency settings. However, comparable results were obtained when this fraction was between $1 \%$ and $10 \%$ (data not shown). This indicates that the results are robust against small changes in stringency. This was also found for the ESC-diff coregulation network mapping. Moreover, to ensure that the method does not lead to false positive results, the c2.cgp and lung-inflammation were scrambled; i.e. the genes were randomly relocated across the gene sets. Applying the algorithm to these sets did not result in a network even for the lowest stringency level (data not shown), confirming that the method is robust against random false positive findings.

Applying our algorithm to the ESC-diff gene set collection with 15196 UGIs results in a network with 927 genes mostly located within two major sub-networks that correspond to early and late differentiation processes stages, respectively. Combining GO-term functional enrichment to MCODE clusters within the network reveals that genes involved in similar biological processes are significantly densely connected, which is in agreement with the premise that such genes are co-regulated. Moreover, this analysis shows that early differentiation events, such as loss of pluripotency, are concentrated in a cluster group at the left side of the network, whereas the right side of the network contains two cluster groups involved in later differentiation phases, one of which is specifically related to muscle and heart function (Fig 2A). The early-late division is supported by overlaying gene expression data from an independent study on ESC cardiomyocyte differentiation time series [177] (Fig 2B). Genes expressed during intermediate time points in that study did not form a separate sub-network, but instead appeared within either of the major sub-networks. This can partially be ascribed to the focus of most literature studies on the differences between undifferentiated and fully differentiated cells, leaving intermediate time point genes underrepresented in the combined gene set collection. Furthermore, whereas pluripotent stem cells or differentiated cardiomyocytes are both stable situations regarding gene expression, the intermediate phase can be described as consecutive waves of gene expression corresponding to sequential transient activation of differentiation programs $[28,29,177]$. Due to their transient expression, such genes are more difficult to identify than those involved in the initial and final phase of the differentiation process and they may therefore have been described in smaller numbers 
$[29,177]$. Both these factors will lead to a relatively smaller number of co-regulation edges among intermediate phase genes, which influences the Cytoscape visualization as its mapping algorithm is developed for grouping together connected genes rather than visualizing data as a time series. Nevertheless, the additional use of the MCODE plugin results in three functional cluster groups that are visually separated and each correspond to a particular differentiation phase.

At the lower left side of Figure $2 \mathrm{~A}$, there is a small sub-network containing a number of genes involved in development, proliferation and pluripotency. These genes could have been expected to occur in the larger sub-network in the upper left side of this figure. The presence of a separate sub-network might indicate a possible common factor influencing the (co-)expression of these genes. However, as the main associated processes are still comparable to that of its larger neighbor except that the functional annotation enrichment of this smaller sub-network is not significant, the relevance of this sub-network should not be overinterpreted. The same can be said for the six groups of two or three genes each in the very lower right of the network visualization. Their occurrence as separate groups is not robust to small changes in the algorithm settings used, and they can be considered as falling just short of being connected to the main network.

The three functional groups in the network contain over 60 genes with a GO annotation organ development as an umbrella term, or more specific terms regarding development of a specific organ or tissue. Apart from those involved in heart or muscle, there was also functional enrichment for the following terms: blood vessel development, ear development, eye development, gland development, immune system development, lung development, nervous system development, skeletal development, as well as some of their daughter terms. Although several genes are annotated to the development of more than one type of organ, muscle and heart (-specific) development-related genes are significantly found in the third group (Table 3), and the second group (Table 2) significantly contains genes (specifically) involved in the development of other organs. Because genes expressed during the intermediate and late time points in the van Dartel [177] study are found in the sub-networks around the second as well as the third group, this indicates that during culture conditions favoring cardiomyocyte differentiation, also other types of tissues are formed.

Other functional analyses showed that the overall network not only contains various development- and heart/muscle-associated genes, but in addition genes involved in cellular proliferation, transcription and also several members of the alpha crystallin/ small heat shock protein family. The first of these processes can easily be understood as the starting point of stem cell differentiation in a culture of proliferating cells. Likewise, transcription factors are necessary to trigger and regulate the developmental changes that occur between proliferating stem cells and fully differentiated cells, which can 
explain why these genes are mainly found in the development-related first and second group, but hardly in the muscle function-related third group. In contrast, this third group contains a number of small heat shock proteins and the structurally related alpha crystallin B. Several members of this chaperonin family have already been associated with muscle contraction or development, and as Hspb3 has not been associated with such a role yet, this might indicate a novel function for this protein. However, although a common relevance of chaperonins in heart function is conceivable, extending this association to $\mathrm{Hspb} 3$ would require additional study and verification, as there is evidence that in heart Cryab and Hspb2 act through different and distinct mechanisms within cardiomyocytes [292].

Of the 927 genes in the network, 327 (35\%) have been described in multiple gene lists, but have not yet been linked to a related developmental process in Gene Ontology or by literature association. Although in most of the studies the genes are only part of a bulk of differentially expressed genes, this combined study shows that there is good evidence to describe them as novel genes involved in ESC differentiation. Furthermore, based on their position in the network, it can already be assumed whether they are mainly expressed at early or late differentiation stages. For 43 of these novel genes, there is even more specific evidence, namely that they are part of densely connected cluster groups within the overall network that are enriched for a relevant biological function.

To determine the in vivo relevance of these 43 genes, we searched the literature as well as the Mouse Genome Informatics (http://www.informatics.jax.org) and the International Knockout Mouse Consortium (http://www.knockoutmouse.org) databases for mouse knockout phenotype data. For twelve genes phenotype data have been described. In the case of four genes, these data are supportive of the putative function in heart or muscle development inferred from the network. The Apobec2 knockout phenotype revealed a role in maintenance of slow/fast muscle fiber-type ratios [293]; Cth deletion results in overproliferation of smooth muscle cells [294]; deletion of the Ptges gene leads to adverse ventricular remodeling after myocardial infarction [295]; and Rrad knockout mice display cardiac hypertrophy [296]. In the case of Rrad, this is further supported by additional human data [296]. For an additional two genes, mouse knockout data are consistent with an early developmental function. Mice homozygous for a 2410146L05Rik (Ooep) null allele exhibit female infertility associated with a failure of embryos to progress beyond the 2 cell stage [297]; for Zfp57, loss of its the zygotic function causes partial neonatal lethality, whereas eliminating both the maternal and zygotic functions of Zfp57 results in a highly penetrant embryonic lethality due to effects on genomic imprinting [298]. Taken together, in vivo knockout data corroborate the putative function for half the genes for which such data are available.

This indicates that the other genes in Table4 also provide interesting candidates for further studies regarding embryonic stem cell cardiac differentiation. Among these, 
Hspb3 and two transcription factors (Rcor2 and Zfp503) are part of pathways enriched within the network, which makes them the mechanistically most conceivable starting points. We compared expression data for these genes across multiple tissues and cell types by means of the BioGPS website (http://biogps.gnf.org) [299, 300]; Hspb3 showed high expression in heart and skeletal muscle, Rcor2 in mouse embryonic stem cell lines, and Zfp503 in multiple tissues. This is in agreement with their proposed biological role, although further study will be required to corroborate their mechanistic and functional importance.

In addition to mechanistic studies of stem cell differentiation, we expect the newly found genes to be potentially useful in applied test systems using ESC differentiation as a model to identify developmental-toxic properties of compounds, such as the Embryonic Stem cell Test (EST) [19]. The implementation of molecular biological approaches in such models may help to improve the prediction accuracy of the model $[111,177]$. Furthermore, it may help in understanding the biology of the model, which is useful for the definition of its applicability domain. Thus, a further knowledge of genes involved in cardiomyocyte differentiation as well as their regulation can help to further optimize such test models for developmental toxicity.

\section{Acknowledgements}

This study was supported by grant MFA6809 from the Dutch technology society foundation STW and by grant nr 050-06-510 from the Netherlands Genomics Initiative/Netherlands Organization for Scientific Research (NWO).

\section{Supplementary data}

Supplementary data associated with this article can be found at doi:10.1089/ scd.2010.0181. 

Dorien A.M. van Dartel, Aldert H. Piersma

Reproductive Toxicology (2011) accepted for publication 
CHAPTER 11

Summary and general discussion 



\section{Summary of main findings}

The EST is an in vitro testing model that has been developed for the prediction of developmental toxicity. Although the EST has shown promising results, the test has not yet been accepted as valid alternative assay for the evaluation of developmental toxicity. As outlined in chapter 1, we anticipated that the implementation of transcriptomics may improve the EST regarding culture duration, end point evaluation, applicability domain definition and predictive capacity. Within this thesis, multiple studies are described that contributed to optimization of the EST. The main findings of these investigations are summarized in Figure 1 and described below.

The experiments described in chapter 2 aimed at unraveling the intertwined relation of proliferation and differentiation in the EST protocol. Experiments showed that end points characteristic for cell viability and proliferation, including EB size, protein concentration and cell cycle distribution, were affected in cultures exposed during the first 3 days of the protocol to non-specific developmental toxicants (5-fluorouracil and 5-bromo-2'-deoxyuridine), whereas the specific developmental toxicants (monobutyl phthalate (MBP) and 6-aminonicotinamide (6-AN)) did not affect these parameters. Furthermore, it was shown that the earliest markers for development are detected around day 3. Together, these findings suggested that for improved assessment of compoundinduced effects on embryonic stem cells (ESC) differentiation as a measure for developmental toxicity, compound exposure should begin following day 3 of culture.

In chapter 3, we further characterized the ESC differentiation process in the EST model via transcriptomic analysis of ESC differentiation cultures. The cultures were sampled 6, 12 and 24h after the onset of differentiation at day 3 and showed a clear induction of well-known developmental markers as a response of ESC differentiation. All genes identified to be induced in ESC differentiation in that study were combined in a gene set ('Van_Dartel_heartdiff_24h'). For detection of subtle gene expression changes, additional analyses based on enrichment of gene sets were performed using gene set collections derived from databases and literature as well as the novel derived gene set 'Van_Dartel_heartdiff_24h'. These analyses confirmed ESC differentiation by downregulation of pluripotency-related gene sets and concomitant up-regulation of development-related gene sets. Furthermore, the novel gene set 'Van_Dartel_heartdiff_24h' showed significant overlap with other developmental-related gene sets, indicating that this set describes early ESC differentiation-related responses. In addition, gene expression regulation upon exposure to the developmental toxicant MBP was studied. Although the concentration of MBP effectively inhibited ESC differentiation as assessed via morphological evaluation, no individual genes were regulated by the treatment. However, gene set enrichment analysis showed that our novel gene set ('Van_Dartel_ heartdiff_24h') was significantly down-regulated, thereby showing direct evidence that 


\section{Chapter 2}

Transition of proliferating pluripotent cells to differentiating cells showed to be a gradual process that starts around day 3 of the EST

\section{Chapter 3}

The novel gene set ('Van_Dartel_heartdiff_24h') described early ESC differentiation and was successful in identifying ESC differentiation inhibition by MBP

\section{Chapter 4}

The continuous process of ESC differentiation was described using the PCA-based 'differentiation track'. This approach was successful in identification of the developmental toxicants MBP and 6-AN

\section{Chapter 5}

The novel gene set 'EST biomarker genes' allowed identification of developmental toxicants using the differentiation track approach

\section{Chapter 6}

The accuracy values for developmental toxicity prediction using the gene set 'Van Dartel_heartdiff 24h' and 'EST biomarke genes' were $83 \%$ and $68 \%$, respectively

\section{Chapter 7}

Flusilazole induced clear concentration-dependent effects on differentiation track deviation as well as on the functional process level. Developmental-related processes were most sensitive to flusilazole exposure

\section{Chapter 8}

Compounds with distinct modes of action were succesfully discriminated using the EST en transcriptomics. Toxicity ranking of compounds within a structural class may be possible

\section{Chapter 9}

A novel gene set was identified using integrated analysis of our data that further improved developmental toxicity prediction using the EST and transcriptomics

\section{Chapter 10}

Using the co-regulation network, 43 genes were identified that are likely involved in development-related processes
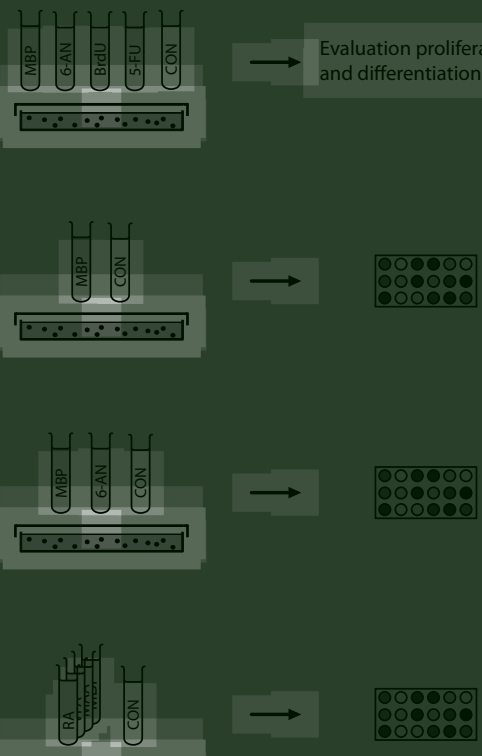

$\because \because \cdots \cdots \cdots \cdot$
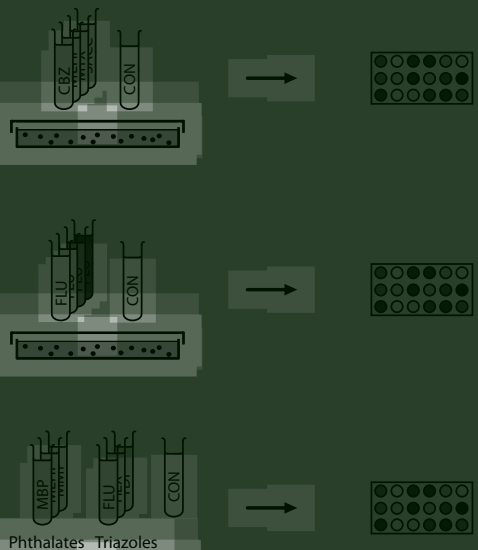

Evaluation proliferation and differentiation

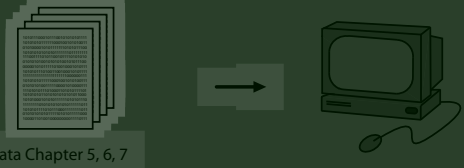

Data Chapter 5, 6, 7

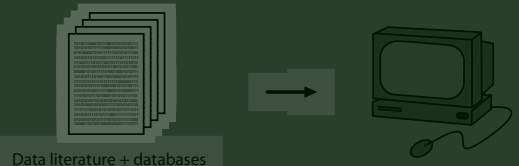

Data literature + databases 
MBP modulated ESC differentiation. This finding was supported by increased expression of pluripotency-, proliferation-, and non-mesodermal differentiation-related gene sets.

In chapter 4, we studied the temporal gene expression regulation in ESC differentiation cultures up to $96 \mathrm{~h}$ after initiation of differentiation. Our analyses revealed both early, midterm and late responses during ESC differentiation that confirmed the behavior of important processes known to be related to development. Down-regulation of genes related to pluripotency and proliferation were identified as early response, and up-regulation genes annotated to differentiation-related processes as late response. In addition, two transiently expressed genes clusters at 24 and $48 \mathrm{~h}$ were identified. Only a few genes of these clusters have been annotated to developmental-related processes, but it was anticipated that these genes are associated to development. We showed that Principal Component Analysis (PCA), using the identified genes regulated during ESC differentiation, could be used to describe the route of ESC differentiation, named the 'differentiation track'. Furthermore, the 'differentiation track' showed to be a successful approach for identification of developmental toxicants in the EST protocol. The developmental toxicants MBP and 6-AN deviated significantly from the time-matched controls on the defined differentiation track, which was in line with the developmental-toxic properties of the compounds.

The study described in chapter $\mathbf{5}$ confirmed the possibility to identify developmental toxicants via the 'differentiation track' approach. Using the obtained gene expression data, a minimum subset of genes was identified to define the differentiation track, which included 26 genes regulated both in ESC differentiation and by exposure to each of the developmental toxicants, named 'EST biomarker genes'. The 'differentiation track' defined by the gene set 'EST biomarker genes' correctly identified the compounds that were under study. Moreover, leave-one-out cross-validation showed a favorable performance of developmental toxicant identification using our gene set approach.

Identification of developmental toxicity using the EST and the gene sets identified in chapter 3 and 5, respectively 'Van_Dartel_heartdiff_24h' and 'EST biomarker genes', was evaluated in chapter 6 by testing 12 additional compounds. The obtained accuracies were 83\% (10/12) for the gene set 'Van_Dartel_heartdiff_24h' and 67\% (8/12) for the gene set 'EST biomarker genes.' Furthermore, it was shown that ESC differentiation inhibition, which is related to compound concentration, correlated with the significance 'differentiation track' deviation. Based upon this finding, it was concluded that for correct prediction of developmental toxicity compound concentration appears an important factor.

The effect of compound concentration on gene expression regulation in ESC differentiation cultures was subject of the study described in chapter 7. The developmental toxicant flusilazole was selected as test compound. This study confirmed that the significance of deviation of compound-exposed cultures of from the defined 'differen- 
tiation track' is concentration-dependent. Furthermore, gene expression profiling in flusilazole-exposed cultures showed alterations in developmentally related processes at all concentrations and preceded ESC differentiation inhibition. Effects of flusilazole exposure on cell-division-related processes were only detected at the highest concentration. In addition, flusilazole's pharmacological mode of action could be identified by the regulation of sterol-synthesis-related processes. Overall, gene expression regulation was identified with increasing magnitude and significance of response at increasing concentrations.

Furthermore, it was studied if gene expression profiling could be used to discriminate compound classes with distinct modes of action. In chapter 8, gene expression data of ESC differentiation cultures exposed to six compounds belonging to two classes, namely phthalates and triazoles, was evaluated. As a proof of principle, three different data analysis approaches demonstrated class-characteristic gene regulation that was useful for discrimination of phthalates and triazoles. Moreover, within the phthalate class, the gene expression profile of the non-developmentally toxic phthalate was dissimilar from that of the developmentally toxic phthalates, which indicates that toxicity ranking of compounds within a structural class may be possible.

Using available transcriptomic data of chapter 5, 6 and 7, a de novo analysis was performed that identified a gene set that resulted in improved prediction of developmental toxicity as described in chapter 9. A large number of the genes within the novel gene set are involved in development and stem-cell-related processes, which illustrated the biological robustness of the set. Using cross-validation, it was confirmed that developmental toxicity prediction using this gene set and the 'differentiation track' approach correlated better with the EST quantitative end point.

In chapter 10, a co-regulation network of ESC differentiation was constructed. To create this network, jointly regulated genes were identified based on their co-occurrence in individual experimental gene sets previously described in literature. This co-regulation network showed two major sub-networks that correspond to early and late differentiation processes stages, respectively. The early-late division was supported by overlaying gene expression data from our independent study on ESC differentiation time series described in chapter 4. Using the co-regulation network several genes not previously associated with the ESC differentiation process were revealed for which a putative novel biological function was assigned. 


\section{General discussion}

Studying differentiation modulation within the EST

A more detailed description of processes active during ESC differentiation contributes to improved definition of the relevance of the EST. In this regard, the intertwined relation of proliferation and differentiation in ESC differentiation has been extensively studied in chapter $\mathbf{2}$ to allow a more specific identification of compound-induced effects on ESC differentiation. This investigation showed that during ESC differentiation proliferation gradually decreases and concomitantly differentiation increases from day 3 onwards, thereby confirming previous findings $[88,89,301]$. Consequently, it has been suggested that when specific effects on differentiation are of interest, such as in the identification of potential developmental toxicants, compound exposure should perhaps more specifically be limited to the differentiation phase of the protocol. This study suggested that to optimally research specific effects of compounds on differentiation modulation, compound exposure should begin following day 3 of culture. These findings are in contrast to the suggestion of the ECVAM/ReProTect report of Marx-stoelting et al that a simple ESC cytotoxicity test may be used to predict developmental toxicity, which was based upon an identified strong correlation between cytotoxicity and effects on ESC differentiation [170]. Within that evaluation, assessment of specific developmental effects of compounds was based on data that applied the classical study design of the EST. Thus, ESC differentiation cultures were exposed throughout the complete ten days of the assay that includes the phase dominated by proliferation. Identification of compound induced effects on functional processes during ESC differentiation has been considered to provide more relevant information for reliable toxicity prediction than cytotoxicity data alone. Consequently, investigations that contribute to elucidation of functional processes within the EST are greatly welcomed. Modern genomic technologies meet this demand of providing a more detailed description of the biology of the model via the possibility to study responses on the whole genome level. Studies applying genomic technologies within the EST may contribute to successful implementation of the EST as an alternative testing method in regulatory toxicity testing.

\section{Implementation of transcriptomics into the EST}

First initiatives on the implementation of molecular techniques in the EST

The first studies that evaluated gene expression regulation as a consequence of compound exposure in the EST were based on a selection of well-known developmental markers. The first study on compound-induced gene expression regulation in the EST focused on the cardiac markers Myh6 and Myh7 and showed that end point objectivation 
of the EST could be obtained by performing gene expression analysis [30]. Later, Pellizer et al. focused on gene regulation of four selected markers that are involved in different stages of cardiomyocyte differentiation, Pou5f1, T, Nkx2-5, and Myh6 [31]. Exposure of the EST cultures to the test compounds lithium and retinoic acid resulted in dissimilar modulation of the dynamic gene expression of the selected genes, indicating the differential mechanism of developmental toxicity of these two compounds. These promising results have stimulated more research that aimed at predicting developmental toxicity in the EST using gene expression analysis.

Characterization of ESC differentiation using a transcriptomic approach Building upon these initial molecular-based studies examining a collection of markers representative of ESC differentiation, recent transcriptomic studies have provided a robust assessment of the dynamics of ESC differentiation. A part of these studies compared gene expression profiles of relatively late stages of differentiated cell cultures with pluripotent ESC [26, $80,144,145,302-304]$. These studies confirmed that the expression pattern in the specialized cell types derived from ESC reflects the functional processes present in fetal cell types. However, the molecular pathways that govern early phase of ESC differentiation are incompletely understood, although recent studies, including our study described in chapter 4, have started to address this issue on the global molecular level [27, 177, 305307]. Together, these studies have shown that early ESC differentiation is characterized by a cascade of time-dependent early, midterm and late gene expression responses. The behavior of important processes known to be related to development have been identified in ESC differentiation cultures, such as down-regulation of pluripotency (including Nanog, Pou5f1 and Sox2) and proliferation (including Ccnb2, Ccnd1, Ccne1, Ccnh, Cdk2) as an early response, and up-regulation of differentiation (including Shh, Gata6, Sox17, Nestin) as a late response $[177,305,306]$. These and other genes were also present in multiple literature-derived gene sets that were used to describe ESC differentiation $[111,177]$, thereby suggesting their functioning in ESC differentiation. In addition, the co-occurrence of genes within these differentiation-related gene sets was used to create a co-regulation network in chapter 10 that included well-known pluripotency and differentiation markers. This co-regulation network showed three sub-networks associated with either early or late responses in ESC differentiation, namely stem cell development, organ development and heart development. Furthermore, the transcriptomic studies that evaluated the gene expression responses in ESC differentiation also revealed transient midterm gene expression responses that included genes that were not yet annotated to developmental-related processes, but were co-expressed with well-known developmental markers $[27,28,177,307]$. A similar observation has been described in chapter 3 in which a novel gene set was identified named 'Van_Dartel_heartdiff_24h' that comprises genes co-expressed with the well-known developmental markers $T$ and Mesp1, but also included genes not previously associated with development. Other 
studies have shown that genes co-expressed with known developmental markers may function in embryonic development since in situ hybridization studies detected expression of many of these transcripts in embryos $[27,135,143]$. These studies suggest that in vitro ESC differentiation monitoring by transcriptomics may effectively mimic gene expression in embryonic development and may be a useful model for studying developmental toxicity.

Identification of developmental toxicants via gene expression alterations in the EST

Our study described in chapter $\mathbf{3}$ as well as other studies have shown that gene expression responses in models of embryonic development upon toxicant exposure may be limited [111, 146, 177]. For that reason, array analysis tools that use data from multiple genes, such as pathway or gene set based analyses, have been applied for the identification of developmental toxicant-induced effects in our subsequent analyses. Such methods have been shown to be more powerful than those based on effects of individual genes $[111,177,308]$. However, the interpretation of regulated gene sets is complex, especially when multiple transiently expressed gene sets are evaluated, such as in ESC differentiation modulation, because individual gene sets only describe a part of the process. To overcome this limitation, a method that allows for a continuous description of ESC differentiation should be applied.

In chapter 4, it was shown that the continuous developmental changes occurring in routine ESC differentiation can be reflected in a 'differentiation track' using principal component analysis (PCA). In a recent transcriptomic study of Sharov et al., a similar approach was used to describe embryonic development [153]. The expression profiles of almost 3000 genes of mouse stem cells and early embryos were used in a PCA and resulted in the identification of developmental timing of the samples. Later, Fang et al. showed that this concept is also successful in describing the development of human embryos during the first third of organogenesis [232]. Besides the use of this approach to visualize and quantify the differentiation status and developmental potency status of cells, this concept is also valuable to identify differentiation modulation by developmental toxicants, using the significance of 'differentiation track' deviation as a measure for developmental toxicity [111, 214].

Initially, in chapter 4, the 'differentiation track' in the EST was described using a large set of genes that were regulated in ESC differentiation, which showed the possibility for identification of developmental toxicants. However, to allow for future robust inexpensive assay development, a further reduction of the number of genes needed to describe the 'differentiation track' was desirable. Besides the identification of the gene set 'Van_Dartel_heartdiff_24h' in chapter 3 that includes 38 genes regulated in early ESC differentiation, the gene set 'EST biomarker genes' was identified in chapter $\mathbf{5}$ that includes 26 genes responsive to ESC differentiation which, in addition, were regulated 
by developmental toxicant exposure. We showed in chapter $\mathbf{5}$ and chapter $\mathbf{6}$ that both gene sets could successfully be applied to define the differentiation track.

The duration of compound exposure at sampling appeared to be an important factor for successful identification of developmental toxicants as shown in chapter 4 . Increased variation at later stages of differentiation together with a less dynamic gene expression profile hampers identification of developmental toxicants at later differentiation stages. On the other hand, the identification of differentiation modulation at relatively early stages of differentiation may be hindered due to limited differentiation progression in the samples isolated early as was observed in chapter 3. Overall, our studies showed that sampling $24 \mathrm{~h}$ after initiation of compound exposure was a good starting point for studying differentiation modulation in the EST.

Within chapter $\mathbf{3}$ and chapter $\mathbf{5}$ it was shown that initial evaluation of developmental toxicity identification showed promising results for both gene sets. However, to ensure confident decision-making, any new predictive test needs to be validated by testing the performance of toxicant identification using a set of additional compounds. Chapter 6 described the validation of the EST in combination with the 'differentiation track' using the 'Van_Dartel_heartdiff_24h' and 'EST biomarker genes' gene set. This validation was performed using gene expression data of 12 and 18 test compounds, respectively, tested at single concentrations. The accuracy values obtained were $83 \%$ for the 'Van_Dartel_heartdiff_24h' gene set and 68\% for the 'EST biomarker genes'. Later, by integrating available EST-transcriptomic data as described in chapter 9, a new analysis identified a gene set that successfully described ESC differentiation and allowed for further improvement of developmental toxicity prediction. Evaluating the performance of this gene set in combination with the 'differentiation track' approach showed that the developmental toxic properties of all 15 compounds that met the inclusion criteria were correctly predicted, which resulted in an overall accuracy of $100 \%$ for these compounds. In perspective of the $78 \%$ accuracy of the validated EST in which compounds were tested in concentration-response design [19], these results are very promising. However, evaluation of additional developmentally toxic compounds, and especially also negative controls, is of importance to confirm the successful performance of the differentiation track-based EST using the novel identified gene set.

Gene expression regulation associates with compound concentration

Several studies have shown that compound concentration dependent assessment of gene expression is of great importance for the identification of toxic properties $[75,190]$. In a series of transcriptomic studies of Naciff et al., gene expression changes on individual genes showed clear concentration-related induction of fold change and number of regulated genes $[49,50,212]$. This finding was confirmed by the studies of Robinson et al. who evaluated gene responses in embryos exposed to metals [213, 251]. Addition- 
ally, in chapter 6 the interdependence of morphological effect size and the extent of gene expression changes was shown within the EST. In chapter 7, we described a comprehensive study that evaluated the concentration-related gene expression regulation of the teratogen flusilazole within the EST confirmed that gene expression regulation was identified with increasing magnitude and significance of response at increasing concentrations [235]. Using the 'differentiation track' approach, the flusilazole-exposed samples showed a concentration-related pattern of deviation from the differentiation track. Furthermore, within this study, functional analyses revealed three major themes affected by flusilazole exposure, namely development, lipid metabolism and cell division. The enrichment of the functional processes related to lipid metabolism and cell division confirmed flusilazole's mode of action and cytotoxic effects, respectively. However, developmental-related processes appeared most sensitive to flusilazole exposure and were identified in the absence of ESC differentiation inhibition as determined by morphological evaluation. Together, these findings indicate that a compound-induced gene expression response is concentration-related in terms of fold change, significance and affected functional processes. In addition, gene expression responses may be more sensitive than and precede morphological effects. Thus for prediction of potential developmental toxicity using the EST, compound concentrations should be carefully selected. We suggest that compounds should be tested below the range of cytotoxicity, because this allows comparison of the relative sensitivity of ESC differentiation modulation as compared to cytotoxicity. For example, a concentration that results in $80 \%$ cell viability can be selected as highest concentration and subsequent concentrations being a fixed fraction of the highest concentration.

The possibility to identify compound induced responses with greater sensitivity using gene expression profiling asks for a definition when to consider gene expression responses as adaptive or adverse. Identification of regulation of a functional process does not necessarily represent an adverse biological response. Consequently, it is not easy to establish adversity of a compound-induced response. In a recent review by Kienhuis et al. on the application of toxicogenomics for risk assessment, it was stated that at present changes in traditional toxicology parameters are leading when deciding whether a cellular response reflect adaptive or adverse effects [309]. An important challenge in the use of toxicogenomics for hazard characterization will be the development of concentration-response modeling tools to determine adversity of the observed compound-induced effect [192].

Furthermore, concentration-response modeling may be used for relative potency ranking of developmental toxicants within the EST, which may ultimately replace the commonly used stratified prediction of developmental toxicity in many in vitro models. The use of relative potency ranking has been described previously for evaluation of relative toxic properties of dioxin-like compounds [310]. An initial study of Peters et 
al. showed promising results of the application of potency ranking of developmental toxicants using the EST [311]. Later, de Jong et al showed that relative potency of a series of homologous compounds, glycol ether alkoxy acid metabolites and valproic acid analogues, tested within the EST, matched in vivo ranking [196, 312]. In addition, developmental toxicity potency ranking may be applied using toxicogenomic data. For example, within the EST, the significance of 'differentiation track' modulation may allow ranking for developmental toxic properties of compounds. However, evaluation of concentration-related gene expression responses of additional compounds with varying developmental toxic potency needs to be studied to confirm the application of the 'differentiation track' for this ranking approach.

Interspecies- and in vitro to in vivo extrapolation

At present the EST is based on a mouse ESC line. It has been demonstrated that interspecies variation may be a problem for assessing developmental toxicity in vivo [313]. The use of hESC [314] in an equivalent of the murine EST would give added value to the safety assessment of compounds, since the data obtained would be more directly applicable to human hazard assessment. Until now, significant limitations that have been associated with hESC cultures regarding maintenance of pluripotency, differentiation ability and handling time hindered the use of hESC. Fortunately, recent studies have obtained promising advancements regarding these aspects that will facilitate the use of hESC in the EST [315]. First steps in establishing a developmental toxicity test method based on hESC have already been taken $[16,17]$ and will eventually make interspecies extrapolation redundant.

Extrapolating in vitro concentrations to the in vivo situation will always be a crucial aspect for all alternative testing models [316]. Within pharmaceutical industry, the use of in vitro to in vivo extrapolation modeling has been widely employed to assess the kinetics of drug candidates [317]. Also within alternative models for assessment of developmental toxicity physiologically based kinetics models have been developed. Recent studies indicate that these kinetic considerations may improve the extrapolation of in vitro results to the in vivo situation and increase the predictive value of in vitro tests $[118,318]$. These evaluations are indispensible for the application of in vitro toxicity data for risk characterization.

Application of computational approaches to further improve the EST

Prediction of developmental toxicity may be further improved by using in silico computational approaches both from the biological and the chemical perspective. Computational models, such as bioinformatics, data-mining, text-mining and modeling endeavors, may cope with the increasing quantity and quality of biologic information of genomic studies $[271,273,319,320]$. Computational approaches are particularly 
useful for systems biology in understanding biological networks under normal conditions, which can subsequently be useful to study the behavior of networks within such models after compound exposure. In addition, computational models can be used for detailed characterization of chemical properties, which may provide insight into factors such as placental transfer and identification of compounds with similar biologic activity. These advantages contribute to the shift of in vivo to cell-based models to study toxicant-induced responses on the pathway level. Therefore, the application of computational approaches in predictive toxicology is among the objectives of the U.S. National Research Council (NRC) report on toxicity testing in the $21^{\text {st }}$ century [192]. In addition, the US Environmental Protection Agency (EPA) ToxCast program promotes the use of computational approaches to develop models that use data of 467 in vitro assays for prediction of compound-induced toxicity in humans.

Meta-analysis in bioinformatics has contributed to identification of putative novel biological function of genes in diverse research areas [267, 271, 273]. In chapter 10, a meta-analysis using published gene lists derived from 42 transcriptomic studies was performed that described gene expression regulation in ESC or embryonic development. This analysis revealed 43 genes that were not previously associated with ESC differentiation but that were co-regulated with established ESC differentiation markers in a network based on individual experimental gene sets. Such integrative analyses reveal putative novel gene function that might not have been discovered without bioinformatics resources. This information may lead to improved gene function annotation that may allow enhanced data interpretation $[177,233]$ and may aid in description of functional pathways, ultimately leading to improved prediction of developmental toxicity. This example clearly indicates the added value of integrated data set analyses of comprehensive large scale collections of both biological and chemical data to support concept-based modeling and statistical computations.

Due to the fact that developmental toxicants are structurally diverse and may have widely distinct activity features [321], the use of one single gene set trained on a fraction of well-documented developmental toxicants may not be capable of identifying developmental toxicants of previously untested compound classes. It has been suggested that a category approach would overcome this difficulty [254, 322]. This approach is supported by the recent finding that toxicogenomic responses of four teratogens in the whole embryo culture model had limited overlap [55]. The overlap of regulated genes by compounds within a category may be more pronounced and these genes may subsequently result in improved predictive power for additional compounds within the category, albeit that this represents a relatively narrow chemical domain of applicability. Furthermore, class-specific gene sets may allow toxicity ranking within compound classes as well as discrimination between compounds with diverse modes of action. As an example, in chapter $\mathbf{8}$, the categorization of phthalates and triazoles has been shown 
within the EST, using gene sets characteristic for the class-specific differentiation modulation. Using three different informatics approaches the distinct gene expression regulation between compounds of the two different classes was evident. Moreover, within the phthalate class, the gene expression of the non-developmentally toxic phthalate was dissimilar from that of the developmentally toxic phthalates, which shows that toxicity ranking of compounds within a structural class may be possible.

The successful use of computational toxicology has been shown in a study of Green et al. that delineated a biological regulatory network regulated in mouse embryonic headfold by ethanol exposure [323]. Another promising example of integration of bioinformatics approaches to understand embryonic development at the systems level and prediction of developmental toxicity is the virtual embryo model that is under development at the US EPA [324]. The first goals of this virtual embryo project are to create a knowledge base and simulation engine that enable in silico reconstruction of key developmental landmarks. Next, critical effects of compound exposure on these networks are simulated and used for developmental toxicity prediction [325]. Such innovative developments in the field of computational toxicology will provide a wealth of additional information that will optimize the application of in vitro methods in developmental toxicity assessment.

When successful biomarker signatures are identified, gene expression evaluation can eventually be performed using customized gene chips, which will reduce experimental costs. In addition, quick experimental designs, such as the 96-well format EST [326], will contribute to enabling faster screening of series of compounds using the EST. Furthermore, wet-lab procedures can be automated, such as RNA isolation, amplification, labeling and hybridization and efficient (semi-) automated data analysis can be used to analyze data and flag potential developmental toxicants [327]. Altogether, these technical advances together with the application of transcriptomic in the EST may move the EST forward to a predictive model with high-throughput.

\section{Regulatory implementation of the EST}

After the introduction in 1997 of the EST as an alternative model for assessing developmental toxicity, over 65 studies have been published that aimed at characterizing or improving the predictive capacity of the EST. Despite these efforts, the EST has still not reached regulatory acceptance at present. Part of this situation may be explained by unrealistically high expectations about the assay [328]. It should be realized that no single in vitro method, even if combined with other tests in a battery approach, will completely correctly predict the developmental toxicity of compounds, simply because each of these methods represents reductionistic models of the complete pregnant human in all its stages of pregnancy [254], and the entire reproductive cycle is more than a sum of 
individual mechanisms represented in in vitro tests. For this reason, a full replacement of in vivo testing strategies by in vitro alternatives is not possible. On the other hand, if crucial mechanisms and end points could be mimicked in well-established in vitro assays and batteries, their use could preclude in vivo testing of compounds emerging as developmental toxicants in vitro, and overall substantially reduce animal testing.

Several critical factors have hampered EST implementation in regulatory toxicology, including kinetics and interspecies extrapolation, as discussed in the previous section. However, the major issues with respect to implementation of the EST are related to the establishment of its applicability domain and predictive capacity.

Applicability domain

The applicability domain of an alternative test has been defined by Hartung et al. [23] as consisting of two essential components. First, the biological mechanism(s) and end point(s) incorporated in the test need to be defined to assess what types of effects it can detect. Compounds that only affect mechanisms and end points outside the applicability domain will be scored as false negatives in the test. The EST has been advocated to detect developmental toxicants in general [19]. This is an optimistically wide definition given that the test is restricted to scoring effects on the number of spontaneously contracting cardiomyocyte foci that have differentiated from embryonic stem cells in culture. A more limited definition of applicability domain would probably be realistic, which may range from relatively simple embryonic stem cell proliferation inhibition via cardiomyocyte differentiation inhibition to a wider spectrum of embryonic cell differentiation. The latter is based on the notion that cardiomyocytes derive from the mesodermal germ layer that is formed by induction between ectoderm and endoderm. Since derivatives of each of the germ layers have been detected in EST cultures [329] it is clear that cardiomyocyte foci counts give only a limited end point of the complexity of differentiation present in EST. With the introduction of omic techniques, developmental toxicology has gained a wealth of innovative tools that allow a more detailed description of the biological end points of the EST as described in this thesis. We have shown that transcriptomic studies have already contributed to improved knowledge about the biological processes active during ESC differentiation. In addition, first initiatives using proteomics have shown the proof of principle that developmental toxicants can be detected via altered protein profiles $[219,256]$. Furthermore, comparison of developmental stage-specific functional processes between embryogenesis in vivo and the EST protocol will characterize the developmental window of EST both in terms of timing as well as developmental pathways covered by this model [330]. The second component of applicability domain as defined by Hartung et al. [23] refers to the chemical domain for which the test can be employed. In the past, validation studies designed to define performance of EST using a group of diverse chemicals in terms of molecular structure and biological mechanism have given 
false hopes as to the general performance of the test for the universe of chemicals [21, 22]. Category approaches may constitute a more promising approach for defining the chemical applicability domain of EST. This class-specific toxicity evaluation has shown successful results for retinoids in the limb bud micromass [331] and for glycol ether alkoxy acid metabolites and valproic acid analogues in the EST [196, 312]. Moreover, these studies showed that the potency ranking of compounds within a class could successfully be extrapolated between EST and the in vivo situation, in some cases aided by kinetic extrapolation methods. This approach could provide more accurate predictions regarding developmental toxicity in vivo and may be particularly useful for prioritization by prescreening of compounds within classes under development in industry.

\section{Predictability}

The predictability of an assay is generally defined as the performance of the assay determined by the percentages of correct positive and negative outcomes within a series of compounds tested [332]. The ECVAM interlaboratory validation study of the EST showed that $78 \%$ of 160 experiments, corresponding to 20 compounds, resulted in correct predictions in predefined classes of non, weak and strong developmental toxicants [19]. Furthermore, these compounds had widely diverse physicochemical properties, which suggested that the observed predictability would hold with testing additional compounds. Paquette et al. subsequently evaluated the accuracy of the EST for in-house and marketed pharmaceuticals, and showed that, although compounds with receptormediated mechanisms were excluded from the validation set, the overall accuracy for marketed pharmaceuticals was $85 \%$ [22]. However, for in-house compounds, the overall accuracy was only $53 \%$, mainly due to bad separation of non from weak developmentally toxic pharmaceuticals. An additional validation study of 14 compounds showed a very low (4/13) predictability [170]. Thus, these validation studies left considerable remaining uncertainty as to the general predictability of EST. The above efforts in defining predictability as reviewed here have suffered from both the absence of a prior definition of the biological domain of EST, as well as from the variety of chemical domains to which the validation test chemicals belonged. In addition, validation was done based on a mathematical prediction model that placed each compound in one of three categories (non, weak, strong developmental toxicants) [197]. The prediction model was derived from the results of a limited set of six compounds, and did not take account of the biological domain of the test. Furthermore, categorical designations of toxicities as used in that study are subjective given that toxic potency is a continuum and thresholds between categories are therefore artificial. We believe that with an appropriate description of applicability domain in terms of its biological and chemical boundaries as discussed above, validation studies such as those done in the past may become superfluous. As an example, an estrogen binding assay has a well described biological domain, and testing 
a limited set of estrogens and non-estrogens suffices for showing that the test is robust $[333,334]$. Therefore, predictability depends largely on the definition of the applicability domain. Transcriptomic studies have already shown promising results in determining the predictive capacity of the EST $[214,335]$. These studies used gene sets within the biological domain of the differentiation processes present in the assay [214, 335]. Further studies will need to expand this line of research in order to improve predictability on the basis of a well-defined applicability domain.

\section{Current and future application of the EST}

For successful regulatory acceptance of the EST, mechanistic validation of the assay is of prime importance. For this, the domain of applicability in terms of developmental pathways needs further elucidation. A detailed definition of active processes within the culture system will allow definition of the biological pathways and stage of embryonic development covered by the model and subsequently may result in reliable identification of developmental toxicants affecting that component of development. Other models, such as those based on differentiation of ESC into other lineages [15, 336], can further complement to the domain of applicability of the EST.

One further way forward in the prediction of developmental toxicity using alternative testing methods is the battery approach [3]. A battery of alternative models, each covering a specific aspect of reproduction or development, in combination may cover large parts of the reproductive cycle, and may improve the detection of reproductive toxicants. In a recent study performed within the $6^{\text {th }}$ European Framework Program Project ReProTect, a ring trial was conducted in which the adverse effects on reproduction and development of ten blinded reproductive toxicants were correctly predicted using a test battery of 14 in vitro assays [337]. This study showed a proof of principle that a battery approach can be successful in predicting effects on fertility and embryonic development. However, it must be noted that the compounds tested were specific strong developmental toxicants and therefore not a realistic reflection of the universe of chemicals.

In future, the battery approach may be used in a test strategy to predict developmental toxicity of unknown compounds, so that compounds with clear adverse effects on one of the end points do not need further in vivo testing. In addition, more adequate testing strategies based on in vitro assays combined with computational modeling may provide detailed information of compound toxicity and may provide mechanistic insights into the compound's mode of action. This information can subsequently be used for prioritization for more in-depth testing, thereby contributing to reduction of experimental animal use. Genomic technologies have the advantages that toxic responses can be studied on the biological pathway level and for this reason may provide a useful approach in modern toxicity prediction. 
Within this review, the progress that has been made in implementing transcriptomics in the EST have been summarized and additional aspects that need improvement to allow a successful use of the EST for hazard identification in regulatory settings are discussed. At present, the EST has its application primarily in in-house hazard identification. To reach regulatory implementation, definition of biological and chemical applicability domain are essential. Additional mechanistic studies such as those utilizing genomic tools are needed that allow further characterization of developmental pathways in the EST protocol. In addition, a comprehensive comparison of the developmental processes active in ESC differentiation with in vivo embryogenesis will allow more precise definition of the developmental pathways and developmental timing covered by the EST. Finally, a detailed definition of the applicability domain of the EST is expected to result in reliable predictability of the EST for the developmental phase covered.

\section{Acknowledgements}

This work was supported by the Dutch technology society foundation STW [grant MFA6809]. 

C

C

(0)

$0+(10)$

?

c

W

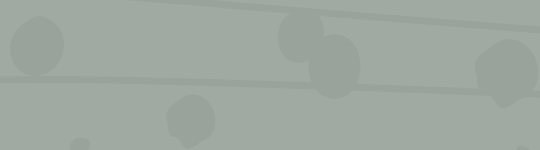

$2012-(1)$

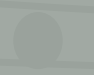

0

(

0

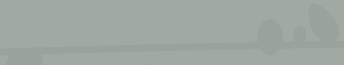

(7)

c

$0^{2}$

U

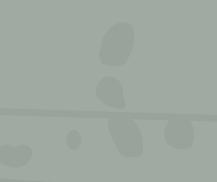

(2)

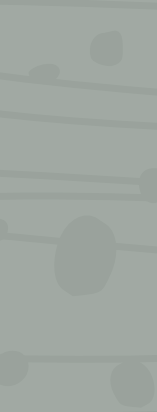

Q 0 
Nederlandse samenvatting 



\section{Inleiding}

In de toxicologie, de leer van giftige stoffen, worden erg veel proefdieren gebruikt om de schadelijkheid van stoffen te voorspellen. Voornamelijk binnen de reproductie- en ontwikkelingstoxicologie worden grote aantallen proefdieren gebruikt, omdat voor deze studies vaak meerdere generaties nodig zijn. Er is grote behoefte aan alternatieve methoden voor het voorspellen van toxiciteit, omdat de huidige manier van onderzoek met behulp van proefdieren ethisch ongewenst is. Bovendien biedt de huidige methode voor toxiciteit voorspelling niet de capaciteit om de toxiciteit van de vele duizenden stoffen met onvolledig toxiciteitsprofiel te evalueren.

Een veelbelovende alternatieve testmethode voor het voorspellen van schadelijkheid van een stof voor de embryonale ontwikkeling (embryotoxiciteit) is een model dat gebaseerd is op differentiatie van embryonale stamcellen (ESC) naar hartspiercellen, de Embryonale Stamcel Test (EST). Verstoring in het proces van embryonale stamceldifferentiatie ten gevolge van blootstelling aan een stof kan gebruikt worden als maat voor embryotoxiciteit. De remming van de hartspierdifferentiatie kan beoordeeld worden onder de microscoop. Een nadeel van deze manier van beoordelen is dat deze subjectief en tijdrovend is. Het doel van dit onderzoek was te bestuderen of een betrouwbare voorspelling voor embryotoxiciteit gedaan kan worden met behulp van de EST in combinatie met transcriptomics. Transcriptomics is een relatief nieuwe techniek waarmee de expressie van wel 20.000 genen in één keer gemeten kan worden. Met deze techniek kunnen veranderingen in genexpressie patronen in blootgestelde differentiërende cellen geïdentificeerd worden. Deze informatie kan vervolgens gebruikt worden voor voorspelling van embryotoxiciteit. De EST in combinatie met transcriptomics zal naar verwachting leiden tot een snellere, goedkopere en meer specifieke proefdiervrije test voor voorspelling van embryotoxiciteit.

\section{Samenvatting}

De eerste studie die uitgevoerd is in het kader van dit proefschrift is beschreven in hoofdstuk 2. Het doel van deze experimenten is om de relatie tussen proliferatie en differentiatie in het protocol van de EST op te helderen. Experimenten lieten zien dat celoverleving en proliferatie waren aangetast in celkweken die gedurende de eerste drie dagen van het protocol waren blootgesteld aan aspecifiek werkende embryotoxische stoffen (5-fluorouracil en 5-bromo-2'-deoxyuridine), terwijl specifiek werkende embryotoxische stoffen (monobutyl phthalaat (MBP) en 6-aminonicotinamide (6-AN)) deze parameters niet beïnvloedden. Een ander experiment toonde aan dat markers voor een vroeg stadium van ontwikkeling gedetecteerd werden rond dag 3. Samen wijzen deze 
bevindingen erop dat voor een verbeterde bepaling van effecten van stoffen op ESC differentiatie de blootstelling het beste kan starten vanaf dag 3 van de EST.

In hoofdstuk 3 was het ESC differentiatie proces in de EST in meer detail gekarakteriseerd met behulp van transcriptomics. De monsters, genomen 6, 12 en 24 uur na initiatie van differentiatie op dag 3 , lieten een duidelijke inductie zien van bekende markers voor ontwikkeling. Dit bevestigde differentiatie van de cellen in de kweken. Alle genen die verhoogd tot expressie kwamen tijdens het differentiatie proces werden samengevoegd in een genenset ('Van_Dartel_heartdiff_24h'). Voor detectie van subtiele genexpressie veranderingen werden additionele analyses uitgevoerd gebaseerd op verrijking van genensets. Hiervoor werden genenset collecties gebruikt die verkregen zijn uit databases en literatuur, maar ook de nieuw beschreven genenset 'Van_Dartel_heartdiff_24h' werd gebruikt. Deze analyses bevestigden ESC differentiatie in de controle kweken door suppressie van genensets gerelateerd aan pluripotentie en een samengaande verhoogde expressie van genensets gerelateerd aan ontwikkeling. Bovendien was er een grote overlap tussen de nieuwe genenset 'Van_Dartel_heartdiff_24h' en andere ontwikkeling gerelateerde genensets. Dit bevestigde dat de nieuwe genenset 'Van_Dartel_heartdiff_24h' de vroege ESC differentiatie beschrijft. Tevens was in deze studie de genexpressie verandering ten gevolge van blootstelling aan de embryotoxische stof MBP bestudeerd. Ondanks dat morfologische evaluatie bevestigde dat de geteste concentratie de ESC differentiatie remde, werd geen enkel gen significant gereguleerd door de blootstelling. Echter, een analyse gebaseerd op verrijking van genensets liet zien dat de expressie van onze nieuwe genenset 'Van_Dartel_heartdiff_24h'significant verlaagd was. Deze vinding toonde direct bewijs dat MBP de ESC differentiatie moduleert. Dit resultaat werd verder ondersteunt door verhoogde expressie van pluripotentie-, proliferatie-, en non-mesodermale differentiatie gerelateerde genensets.

De genexpressie regulatie in ESC differentiatie in de tijd werd bestudeerd in hoofdstuk 4. De analyses onthulden zowel vroege, als middel en late genexpressie regulatie tijdens ESC differentiatie. Deze resultaten bevestigden het gedrag van bekende processen die gerelateerd zijn aan ontwikkeling; als vroege respons werd de expressie van genen gerelateerd aan pluripotentie en proliferatie verlaagd en als late respons werd de expressie van genen gerelateerd aan differentiatie verhoogd. Tevens werden ook twee clusters geïdentificeerd met een tijdelijke expressie op 24 en 48 uur. Het is aannemelijk dat de genen in deze twee genen clusters een rol spelen in ontwikkeling, toch is dit voor slechts een klein deel van deze genen bekend. We hebben laten zien dat Principal Component Analyse (PCA), gebruik makende van de genen die gereguleerd werden tijdens ESC differentiatie, gebruikt kon worden om het traject van ESC differentiatie te beschrijven. Dit traject werd de 'differentiation track' genoemd. De 'differentiation track' was een nuttige benadering om embryotoxische stoffen te identificeren. De stoffen MBP 
en 6-AN weken significant af van de differentiation track, dit was in overeenstemming met de embryotoxische eigenschappen van deze stoffen.

De studie beschreven in hoofdstuk 5 bevestigde de mogelijkheid om embryotoxische stoffen te identificeren met behulp van de 'differentiation track' benadering. Vervolgens werd een kleine set bestaande uit 26 genen geïdentificeerd in deze studie. Deze 26 genen werden zowel in ESC differentiatie als door blootstelling aan embryotoxische stoffen gereguleerd. Samen vormden deze genen de set 'EST biomarker genes'. De stoffen die geselecteerd waren in deze studie werden correct geïdentificeerd via de 'differentiation track' benadering gebaseerd op de 'EST biomarker genes'. Bovendien toonde cross validatie aan dat deze bevindingen erg robuust waren.

Identificatie van embryotoxische stoffen met behulp van de EST en de genensets beschreven in hoofdstuk 3 en 5, respectievelijk 'Van_Dartel_heartdiff_24h' en 'EST biomarker genes' was geëvalueerd in hoofdstuk 6 door het testen van 12 extra stoffen. De genenset 'Van_Dartel_heartdiff_24h' voorspelde 83\% (10/12) van de stoffen goed. De goed voorspellende waarde was $67 \%$ (8/12) voor de genenset 'EST biomarker genes'. Tevens werd aangetoond dat de remming van ESC differentiatie, wat gerelateerd is aan de concentratie van de stof, correleerde met de significantie van afwijking van de 'differentiation track'. Vanwege deze bevinding concludeerden we dat voor een juiste voorspelling van embryotoxiciteit de concentratie van de stof een grote rol speelt.

Het effect van concentratie op genexpressie regulatie in ESC differentiatie kweken was het onderwerp van de studie beschreven in hoofdstuk 7. De embryotoxische stof flusilazole werd geselecteerd als teststof. Deze studie bevestigde dat de significantie van afwijking van blootgestelde kweken van de 'differentiation track' afhankelijk is van de concentratie. Tevens liet deze studie zien dat effecten op processen gerelateerd aan ontwikkeling gedetecteerd werden bij alle geteste concentraties van flusilazole, zelfs nog voordat er remming van ESC differentiatie op het morfologisch eindpunt gevonden was. Bij de hoogst geteste concentratie werd een verstorend effect op celdeling gerelateerde processen gevonden. Tevens kon het farmacologische werkingsmechanisme van flusilazole, namelijk verstoring van sterol biosynthese, geïdentificeerd worden op genexpressie niveau. Onze data liet zien dat tengevolge van oplopende concentraties de genexpressie regulatie duidelijker te detecteren was door significantere expressie en sterkere regulatie van genen.

De mogelijkheid om verschillende klassen van stoffen met verschillende werkingsmechanismen te onderscheiden, met behulp van transcriptomic analyses, is beschreven in hoofdstuk 8. Voor deze studie is genexpressie data van zes verschillende stoffen behorend tot twee stof klassen gebruikt, namelijk phthalaten en triazolen. Drie verschillende data analyse methoden toonden aan dat de karakteristieke genexpressie regulatie per klasse gebruikt kon worden om phthalaten en triazolen te discrimineren. Bovendien lieten onze analyses zien dat de niet embryotoxische phthalaat een ander genexpressie 
profiel opleverde dan de embryotoxische phthalaten. Dit wijst er op dat toxiciteitsranking van stoffen binnen een klasse mogelijk zou kunnen zijn.

Een nieuwe analyse met de transcriptomic data van hoofdstuk 5, 6 en 7 werd uitgevoerd en beschreven in hoofdstuk 9. Deze analyse resulteerde in de identificatie van een genenset die een verbeterde voorspelling voor embryotoxiciteit liet zien. Het merendeel van de genen was gerelateerd aan processen die karakteristiek zijn voor ontwikkeling en stamcellen, wat de biologische robuustheid van de set illustreerde. Door middel van cross validatie bevestigde deze studie dat de voorspelling van embryotoxiciteit met deze genenset en de 'differentiation track' benadering een verbeterde correlatie opleverde met het kwantitatieve eindpunt van de EST.

In hoofdstuk 10 werd een co-regulatie netwerk geconstrueerd door identificatie van genen die gezamenlijk tot expressie komen. Genensets die eerder werden beschreven in de literatuur werden gebruikt om het netwerk te maken. Hierin waren twee grote subnetwerken aanwezig, waarvan er één overeenkwam met processen die actief zijn tijdens een vroeg stadium van differentiatie en het andere kwam overeen met processen die actief zijn tijdens late stadia van differentiatie. Deze scheiding van vroege en late differentiatie werd ondersteund door de genexpressie data van onze onafhankelijke studie, beschreven in hoofdstuk 4, te koppelen aan het netwerk. Het co-regulatie netwerk bevatte verschillende genen die niet eerder geassocieerd waren met ESC differentiatie, maar waaraan een vermoedelijk nieuwe biologische functie is toegeschreven.

\section{Conclusie}

Uit de resultaten van dit proefschrift kan worden geconcludeerd dat embryotoxiciteit objectiever kan worden vastgesteld met de transcriptomics gebaseerde EST vergeleken met de 'klassieke' EST waar morfologische evaluatie een van de eindpunten is. Transcriptomics is een gevoeligere methode om de verstoring van ESC differentiatie te detecteren. Hierdoor is het mogelijk modulatie van ESC differentiatie al kort na aanvang van de blootstelling aan te tonen. Het gebruik van een genenset, die de dynamische fase van ESC differentiatie beschrijft, in combinatie met de beschreven 'differentiation track' methode heeft aangetoond een veelbelovende benadering te zijn om de verstoring op ESC differentiatie te detecteren. Het detecteren van embryotoxische verbindingen is echter afhankelijk van de gekozen test-concentratie.

Op dit moment wordt de EST voornamelijk gebruikt voor identificatie van toxiciteit. Om de test geschikt te maken voor risicobeoordeling is een uitgebreidere definitie van het toepassingsdomein en de voorspellende capaciteit van de test essentieel. In het bijzonder mechanistische validatie is een zeer belangrijk aandachtspunt. Hiervoor moet het toepassingsdomein van de EST met betrekking tot de ontwikkelings pathways die 
aanwezig zijn in de EST nader opgehelderd worden. Een gedetailleerde definitie van deze actieve processen in de EST zal bijdragen aan een definitie van de biologische pathways en het stadium van embryonale ontwikkeling dat gedekt wordt door de EST. Deze informatie zal uiteindelijk leiden tot betrouwbare identificatie van embryotoxische stoffen die dat specifieke stadium van ontwikkeling beïnvloeden. 
C

C

(0)

$0+(10)$

?

c

W

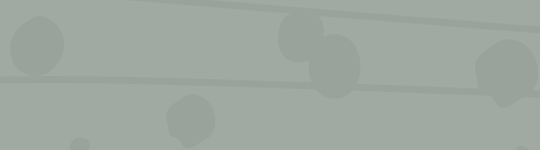

$2012-(1)$

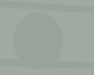

0

(

0

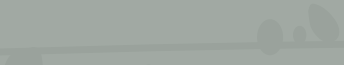

(7)

c

$0^{2}$

U

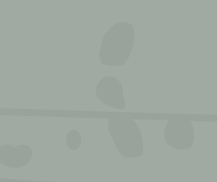

(2)

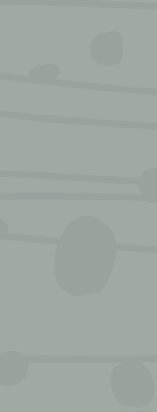

Q 0 
References 

1 Lenz W. A short history of thalidomide embryopathy. Teratology. 1988;38:203-15.

2 Russell WMS, Burch RL. The principles of humane experimental technique. London Methuen; 1959.

3 Hofer T, Gerner I, Gundert-Remy U, Liebsch M, Schulte A, Spielmann H, et al. Animal testing and alternative approaches for the human health risk assessment under the proposed new European chemicals regulation. Arch Toxicol. 2004;78:549-64.

4 Spielmann H, Pohl I, Doring B, Liebsch m, Moldenbauer F. The Embryonic Stem cell Test (EST), an in vitro embryotoxicity test using two permanent cell lines: 3T3 fibroblasts and embryonic stem cells. In vitro Toxicology. 1997;10:119-27.

5 Rudnicki MA, McBurney MW. Cell culture methods and induction of differentiation of embryonal carcinoma cell lines. In: Robertson E, editor. Teratocarcinoma and embryonic stem cells: a practical approach. Washington D.C.: IRL Press; 1987. p. 19-49.

6 Doetschman TC, Eistetter H, Katz M, Schmidt W, Kemler R. The in vitro development of blastocystderived embryonic stem cell lines: formation of visceral yolk sac, blood islands and myocardium. J Embryol Exp Morphol. 1985;87:27-45.

7 ECVAM. INVITTOX protocol 113. http://ecvam-dbalm.jrc.ec.europa.eu. Last accessed: Dec 2010.

8 Kahan BW, Ephrussi B. Developmental potentialities of clonal in vitro cultures of mouse testicular teratoma. J Natl Cancer Inst. 1970;44:1015-36.

9 Martin GR, Evans MJ. The morphology and growth of a pluripotent teratocarcinoma cell line and its derivatives in tissue culture. Cell. 1974;2:163-72.

10 Piersma AH, Haakmat AS, Hagenaars AM. In vitro assays for the developmental toxicity of xenobiotic compounds using differentiating embryonal carcinoma cells in culture. Toxicol in vitro. 1993; 7:615-21.

11 Wobus AM, Boheler KR. Embryonic stem cells: prospects for developmental biology and cell therapy. Physiol Rev. 2005;85:635-78.

12 Evans MJ, Kaufman MH. Establishment in culture of pluripotential cells from mouse embryos. Nature. 1981;292:154-6.

13 Martin GR. Isolation of a pluripotent cell line from early mouse embryos cultured in medium conditioned by teratocarcinoma stem cells. Proc Natl Acad Sci U S A. 1981;78:7634-8.

14 Williams RL, Hilton DJ, Pease S, Willson TA, Stewart CL, Gearing DP, et al. Myeloid leukaemia inhibitory factor maintains the developmental potential of embryonic stem cells. Nature. 1988; 336:684-7.

15 Theunissen PT, Schulpen SH, van Dartel DAM, Hermsen SAB, van Schooten FJ, Piersma AH. An abbreviated protocol for multilineage neural differentiation of murine embryonic stem cells and its perturbation by methyl mercury. Reprod Toxicol. 2010;29:383-92.

16 Adler S, Pellizzer C, Hareng L, Hartung T, Bremer S. First steps in establishing a developmental toxicity test method based on human embryonic stem cells. Toxicol In vitro. 2007;22:200-11.

17 West PR, Weir AM, Smith AM, Donley EL, Cezar GG. Predicting human developmental toxicity of pharmaceuticals using human embryonic stem cells and metabolomics. Toxicol Appl Pharmacol. 2010;247:18-27.

18 Smith MK, Kimmel GL, Kochhar DM, Shepard TH, Spielberg SP, Wilson JG. A selection of candidate compounds for in vitro teratogenesis test validation. Teratog Carcinog Mutagen. 1983;3:461-80.

19 Genschow E, Spielmann H, Scholz G, Pohl I, Seiler A, Clemann N, et al. Validation of the Embryonic Stem cell Test in the international ECVAM validation study on three in vitro embryotoxicity tests. Altern Lab Anim. 2004;32:209-44. 
20 Genschow E, Spielmann H, Scholz G, Seiler A, Brown N, Piersma A, et al. The ECVAM international validation study on in vitro embryotoxicity tests: results of the definitive phase and evaluation of prediction models. European Centre for the Validation of Alternative Methods. Altern Lab Anim. 2002;30:151-76.

21 Chapin R, Stedman D, Paquette J, Streck R, Kumpf S, Deng S. Struggles for equivalence: In vitro developmental toxicity model evolution in pharmaceuticals in 2006. Toxicol In vitro. 2007;21: 1545-51.

22 Paquette JA, Kumpf SW, Streck RD, Thomson JJ, Chapin RE, Stedman DB. Assessment of the Embryonic Stem cell Test and application and use in the pharmaceutical industry. Birth Defects Res B Dev Reprod Toxicol. 2008;83:104-11.

23 Hartung T, Bremer S, Casati S, Coecke S, Corvi R, Fortaner S, et al. A modular approach to the ECVAM principles on test validity. Altern Lab Anim. 2004;32:467-72.

24 Bremer S, Dooren van M, Paparella M, Kolossov E, Fleischmann B, Hescheler J. Effects of embryotoxic chemicals on the in vitro differentiation of genetically engineered embryonic stem cells into cardiac cells. Toxicol in Vitro. 1999;13:645-50.

25 Seiler A, Visan A, Buesen R, Genschow E, Spielmann H. Improvement of an in vitro stem cell assay for developmental toxicity: the use of molecular endpoints in the Embryonic Stem cell Test. Reprod Toxicol. 2004;18:231-40.

26 Doss MX, Winkler J, Chen S, Hippler-Altenburg R, Sotiriadou I, Halbach M, et al. Global transcriptome analysis of murine embryonic stem cell-derived cardiomyocytes. Genome Biol. 2007;8:R56.

27 Terami H, Hidaka K, Shirai M, Narumiya H, Kuroyanagi T, Arai Y, et al. Efficient capture of cardiogenesis-associated genes expressed in ES cells. Biochem Biophys Res Commun. 2007;355:47-53.

28 Beqqali A, Kloots J, Ward-van Oostwaard D, Mummery C, Passier R. Genome-wide transcriptional profiling of human embryonic stem cells differentiating to cardiomyocytes. Stem Cells. 2006;24: 1956-67.

29 Bruce SJ, Gardiner BB, Burke LJ, Gongora MM, Grimmond SM, Perkins AC. Dynamic transcription programs during ES cell differentiation towards mesoderm in serum versus serum-freeBMP4 culture. BMC Genomics. 2007;8:365.

30 Bigot K, de Lange J, Archer G, Clothier R, Bremer S. The relative semi-quantification of mRNA expression as a useful toxicological endpoint for the identification of embryotoxic/teratogenic substances. Toxicol in Vitro. 1999;13:619-23.

31 Pellizzer C, Adler S, Corvi R, Hartung T, Bremer S. Monitoring of teratogenic effects in vitro by analysing a selected gene expression pattern. Toxicol In vitro. 2004;18:325-35.

32 Jergil M. Pluripotent stem cells of embryonic origin - applications in developmental toxicology. Uppsala: Uppsala Universitet; 2009.

33 Kramer JA, Curtiss SW, Kolaja KL, Alden CL, Blomme EA, Curtiss WC, et al. Acute molecular markers of rodent hepatic carcinogenesis identified by transcription profiling. Chem Res Toxicol. 2004;17: 463-70.

34 Nie AY, McMillian M, Parker JB, Leone A, Bryant S, Yieh L, et al. Predictive toxicogenomics approaches reveal underlying molecular mechanisms of nongenotoxic carcinogenicity. Mol Carcinog. 2006;45:914-33.

35 Thomas RS, Pluta L, Yang L, Halsey TA. Application of genomic biomarkers to predict increased lung tumor incidence in 2-year rodent cancer bioassays. Toxicol Sci. 2007;97:55-64.

36 Fielden MR, Brennan R, Gollub J. A gene expression biomarker provides early prediction and mechanistic assessment of hepatic tumor induction by nongenotoxic chemicals. Toxicol Sci. 2007;99:90-100. 
37 Ellinger-Ziegelbauer $\mathrm{H}$, Gmuender $\mathrm{H}$, Bandenburg A, Ahr HJ. Prediction of a carcinogenic potential of rat hepatocarcinogens using toxicogenomics analysis of short-term in vivo studies. Mutat Res. 2008;637:23-39.

38 Lam SH, Mathavan S, Tong Y, Li H, Karuturi RK, Wu Y, et al. Zebrafish whole-adult-organism chemogenomics for large-scale predictive and discovery chemical biology. PLoS Genet. 2008;4: e1000121.

39 Baken KA, Pennings JL, Jonker MJ, Schaap MM, de Vries A, van Steeg $H$, et al. Overlapping gene expression profiles of model compounds provide opportunities for immunotoxicity screening. Toxicol Appl Pharmacol. 2008;226:46-59.

40 Patterson RM, Germolec DR. Gene expression alterations in immune system pathways following exposure to immunosuppressive chemicals. Ann N Y Acad Sci. 2006;1076:718-27.

41 Yang L, Kemadjou JR, Zinsmeister C, Bauer M, Legradi J, Muller F, et al. Transcriptional profiling reveals barcode-like toxicogenomic responses in the zebrafish embryo. Genome Biol. 2007;8: R227.

42 Jessen BA, Mullins JS, De Peyster A, Stevens GJ. Assessment of hepatocytes and liver slices as in vitro test systems to predict in vivo gene expression. Toxicol Sci. 2003;75:208-22.

43 Boess F, Kamber M, Romer S, Gasser R, Muller D, Albertini S, et al. Gene expression in two hepatic cell lines, cultured primary hepatocytes, and liver slices compared to the in vivo liver gene expression in rats: possible implications for toxicogenomics use of in vitro systems. Toxicol Sci. 2003;73: 386-402.

44 Kienhuis AS. Prediction of hepatotoxicants responses in humans - a toxicogenomics-based parallelogram approach. Maastricht: Maastricht University; 2008.

45 Burczynski ME, McMillian M, Ciervo J, Li L, Parker JB, Dunn RT, 2nd, et al. Toxicogenomics-based discrimination of toxic mechanism in HepG2 human hepatoma cells. Toxicol Sci. 2000;58:399-415.

46 Yang Y, Abel SJ, Ciurlionis R, Waring JF. Development of a toxicogenomics in vitro assay for the efficient characterization of compounds. Pharmacogenomics. 2006;7:177-86.

47 Tsujimura K, Asamoto M, Suzuki S, Hokaiwado N, Ogawa K, Shirai T. Prediction of carcinogenic potential by a toxicogenomic approach using rat hepatoma cells. Cancer Sci. 2006;97:1002-10.

48 Vandebriel RJ, Pennings JL, Baken KA, Pronk TE, Boorsma A, Gottschalk R, et al. Keratinocyte gene expression profiles discriminate sensitizing and irritating compounds. Toxicol Sci. 2010;117:81-9.

49 Naciff JM, Hess KA, Overmann GJ, Torontali SM, Carr GJ, Tiesman JP, et al. Gene expression changes induced in the testis by transplacental exposure to high and low doses of 17\{alpha\}-ethynyl estradiol, genistein, or bisphenol A. Toxicol Sci. 2005;86:396-416.

50 Naciff JM, Torontali SM, Overmann GI, Carr GJ, Tiesman JP, Daston GP. Evaluation of the gene expression changes induced by 17-alpha-ethynyl estradiol in the immature uterus/ovaries of the rat using high density oligonucleotide arrays. Birth Defects Res B Dev Reprod Toxicol. 2005;74: 164-84.

51 Goetz AK, Dix DJ. Mode of action for reproductive and hepatic toxicity inferred from a genomic study of triazole antifungals. Toxicol Sci. 2009;110:449-62.

52 Jergil M, Kultima K, Gustafson AL, Dencker L, Stigson M. Valproic acid-induced deregulation in vitro of genes associated in vivo with neural tube defects. Toxicol Sci. 2009;108:132-48.

53 Robinson JF, Griffith WC, Yu X, Hong S, Kim E, Faustman EM. Methylmercury induced toxicogenomic response in C57 and SWV mouse embryos undergoing neural tube closure. Reprod Toxicol. 2010;30:284-91. 
54 Robinson JF, Yu X, Hong S, Griffith WC, Beyer R, Kim E, et al. Cadmium-induced differential toxicogenomic response in resistant and sensitive mouse strains undergoing neurulation. Toxicol Sci. 2009;107:206-19.

55 Robinson JF, van Beelen VA, Verhoef A, Renkens MF, Luijten M, van Herwijnen MH, et al. Embryotoxicant-specific transcriptomics responses in rat postimplantation whole embryo culture. Toxicol Sci. 2010;118:675-85.

56 Slonim DK. From patterns to pathways: gene expression data analysis comes of age. Nat Genet. 2002;32 Suppl:502-8.

57 Eisen MB, Spellman PT, Brown PO, Botstein D. Cluster analysis and display of genome-wide expression patterns. Proc Natl Acad Sci U S A. 1998;95:14863-8.

58 Pearson K. On lines and planes of closest fit to systems of points in space. Philosophical Magazine. 1901;2:559-72.

59 Tamayo P, Slonim D, Mesirov J, Zhu Q, Kitareewan S, Dmitrovsky E, et al. Interpreting patterns of gene expression with self-organizing maps: methods and application to hematopoietic differentiation. Proc Natl Acad Sci U S A. 1999;96:2907-12.

60 Hayes KR, Bradfield CA. Advances in toxicogenomics. Chem Res Toxicol. 2005;18:403-14.

61 Ashburner M, Ball CA, Blake JA, Botstein D, Butler H, Cherry JM, et al. Gene ontology: tool for the unification of biology. The Gene Ontology Consortium. Nat Genet. 2000;25:25-9.

62 GO. http://geneontology.org. Last accessed: Dec 2010.

63 NCBI-GEO. http://www.ncbi.nlm.nih.gov/geo. Last accessed: Dec 2010.

64 Mattingly CJ, Rosenstein MC, Colby GT, Forrest JN, Jr., Boyer JL. The Comparative Toxicogenomics Database (CTD): a resource for comparative toxicological studies. J Exp Zool A Comp Exp Biol. 2006;305:689-92.

65 CTD. http://ctd.mdibl.org. Last accessed: Dec 2010.

66 DAVID. http://david.abcc.ncifcrf.gov/home.jsp. Last accessed: Dec 2010.

67 Dennis G, Jr., Sherman BT, Hosack DA, Yang J, Gao W, Lane HC, et al. DAVID: Database for Annotation, Visualization, and Integrated Discovery. Genome Biol. 2003;4:P3.

68 ErmineJ. http://www.bioinformatics.ubc.ca/ermineJ. Last accessed: Dec 2010.

69 Lee HK, Braynen W, Keshav K, Pavlidis P. ErmineJ: tool for functional analysis of gene expression data sets. BMC Bioinformatics. 2005;6:269.

70 Currie RA, Bombail V, Oliver JD, Moore DJ, Lim FL, Gwilliam V, et al. Gene ontology mapping as an unbiased method for identifying molecular pathways and processes affected by toxicant exposure: application to acute effects caused by the rodent non-genotoxic carcinogen diethylhexylphthalate. Toxicol Sci. 2005;86:453-69.

71 GenMAPP. http://www.genmapp.org. Last accessed: Dec 2010.

72 KEGG. http://www.genome.jp/kegg/pathway.html. Last accessed: Dec 2010.

73 GeneGo. http://www.genego.com. Last accessed: Dec 2010.

74 Ingenuity. http://ingenuity.com. Last accessed: Dec 2010.

75 Baken KA, Arkusz J, Pennings JL, Vandebriel RJ, van Loveren H. In vitro immunotoxicity of bis(trin-butyltin)oxide (TBTO) studied by toxicogenomics. Toxicology. 2007;237:35-48.

76 Xu EY, Perlina A, Vu H, Troth SP, Brennan RJ, Aslamkhan AG, et al. Integrated pathway analysis of rat urine metabolic profiles and kidney transcriptomic profiles to elucidate the systems toxicology of model nephrotoxicants. Chem Res Toxicol. 2008;21:1548-61.

77 Moreira EG, Yu X, Robinson JF, Griffith W, Hong SW, Beyer RP, et al. Toxicogenomic profiling in maternal and fetal rodent brains following gestational exposure to chlorpyrifos. Toxicol Appl Pharmacol. 2010;245:310-25. 
78 FunGenES. http://biit.cs.ut.ee/fungenes. Last accessed: Dec 2010.

79 Schulz H, Kolde R, Adler P, Aksoy I, Anastassiadis K, Bader M, et al. The FunGenES database: a genomics resource for mouse embryonic stem cell differentiation. PLoS One. 2009;4:e6804.

80 Zimmer B, Kuegler PB, Baudis B, Genewsky A, Tanavde V, Koh W, et al. Coordinated waves of gene expression during neuronal differentiation of embryonic stem cells as basis for novel approaches to developmental neurotoxicity testing. Cell Death Differ. 2010;doi:10.1038/cdd.2010.109.

81 Bourillot PY, Savatier P. Kruppel-like transcription factors and control of pluripotency. BMC Biol. 2010;27:125.

82 Subramanian A, Tamayo P, Mootha VK, Mukherjee S, Ebert BL, Gillette MA, et al. Gene set enrichment analysis: a knowledge-based approach for interpreting genome-wide expression profiles. Proc Natl Acad Sci U S A. 2005;102:15545-50.

83 T-profiler. http://www.t-profiler.org. Last accessed: Dec 2010.

84 Flint OP, Orton TC. An in vitro assay for teratogens with cultures of rat embryo midbrain and limb bud cells. Toxicol Appl Pharmacol. 1984;76:383-95.

85 Piersma AH, Genschow E, Verhoef A, Spanjersberg MQ, Brown NA, Brady M, et al. Validation of the postimplantation rat whole-embryo culture test in the international ECVAM validation study on three in vitro embryotoxicity tests. Altern Lab Anim. 2004;32:275-307.

86 Rubinstein AL. Zebrafish assays for drug toxicity screening. ExpertOpinDrug Metab Toxicol. 2006; 2:231-40.

87 Leahy A, Xiong JW, Kuhnert F, Stuhlmann H. Use of developmental marker genes to define temporal and spatial patterns of differentiation during embryoid body formation. J Exp Zool. 1999;284: 67-81.

88 Boheler KR, Czyz J, Tweedie D, Yang HT, Anisimov SV, Wobus AM. Differentiation of pluripotent embryonic stem cells into cardiomyocytes. Circ Res. 2002;91:189-201.

89 White J, Dalton S. Cell cycle control of embryonic stem cells. Stem Cell Rev. 2005;1:131-8.

90 Brown NA. Selection of test chemicals for the ECVAM international validation study on in vitro embryotoxicity tests. Altern Lab Anim. 2002;30:177-98.

91 Ema M, Amano H, Ogawa Y. Characterization of the developmental toxicity of di-n-butyl phthalate in rats. Toxicology. 1994;86:163-74.

92 Ema M, Kurosaka R, Amano H, Ogawa Y. Developmental toxicity evaluation of mono-n-butyl phthalate in rats. Toxicol Lett. 1995;78:101-6.

93 Ema M, Murai T, Itami T, Kawasaki H. Evaluation of the teratogenic potential of the plasticizer butyl benzyl phthalate in rats. J Appl Toxicol. 1990;10:339-43.

94 Chernoff N, Kavlock RJ. An in vivo teratology screen utilizing pregnant mice. J Toxicol Environ Health. 1982;10:541-50.

95 Matschke GH, Fagerstone KA. Teratogenic effects of 6-aminonicotinamide in mice. J Toxicol Environ Health. 1977;3:735-43.

96 Turbow MM, Chamberlain JG. Direct effects of 6-aminonicotinamide on the developing rat embryo in vitro and in vivo. Teratology. 1968;1:103-8.

97 Danenberg PV, Langenbach RJ, Heidelberger C. Structures of reversible and irreversible complexes of thymidylate synthetase and fluorinated pyrimidine nucleotides. Biochemistry. 1974;13: 926-33.98 Santi DV, McHenry CS, Sommer H. Mechanism of interaction of thymidylate synthetase with 5-fluorodeoxyuridylate. Biochemistry. 1974;13:471-81.

99 Hakala MT. Mode of action of 5-bromodeoxyuridine on mammalian cells in culture. J Biol Chem. 1959;234:3072-6. 
100 Hakala MT. Effect of 5-bromodeoxyuridine incorporation on survival of cultured mammalian cells. Biochim Biophys Acta. 1962;61:815-23.

101 Packard DS, Jr., Skalko RG, Menzies RA. Growth retardation and cell death in mouse embryos following exposure to the teratogen bromodeoxyuridine. Exp Mol Pathol. 1974;21:351-62.

102 De Smedt A, Steemans M, De Boeck M, Peters AK, van der Leede BJ, Van Goethem F, et al. Optimisation of the cell cultivation methods in the Embryonic Stem cell Test results in an increased differentiation potential of the cells into strong beating myocard cells. Toxicol In vitro. 2008;22: 1789-96.

103 Slob W. Dose-response modeling of continuous endpoints. Toxicol Sci. 2002;66:298-312.

104 Showell C, Binder O, Conlon FL. T-box genes in early embryogenesis. Dev Dyn. 2004;229:201-18

105 Wilkinson DG, Bhatt S, Herrmann BG. Expression pattern of the mouse $\mathrm{T}$ gene and its role in mesoderm formation. Nature. 1990;343:657-9.

106 Yamauchi-Takihara K, Sole MJ, Liew J, Ing D, Liew CC. Characterization of human cardiac myosin heavy chain genes. Proc Natl Acad Sci U S A. 1989;86:3504-8.

107 AppliedBiosystems. http://www3.appliedbiosystems.com/cms/groups/mcb_support/documents /generaldocuments/cms_042380.pdf. Last accessed: Dec 2010.

108 Fluckiger AC, Marcy G, Marchand M, Negre D, Cosset FL, Mitalipov S, et al. Cell cycle features of primate embryonic stem cells. Stem Cells. 2006;24:547-56.

109 Savatier P, Huang S, Szekely L, Wiman KG, Samarut J. Contrasting patterns of retinoblastoma protein expression in mouse embryonic stem cells and embryonic fibroblasts. Oncogene. 1994;9: 809-18.

110 Stead E, White J, Faast R, Conn S, Goldstone S, Rathjen J, et al. Pluripotent cell division cycles are driven by ectopic Cdk2, cyclin A/E and E2F activities. Oncogene. 2002;21:8320-33.

111 van Dartel DAM, Pennings JLA, Hendriksen PJ, van Schooten FJ, Piersma AH. Early gene expression changes during embryonic stem cell differentiation into cardiomyocytes and their modulation by monobutyl phthalate. Reprod Toxicol. 2009;27:93-102.

112 Rohwedel J, Sehlmeyer U, Shan J, Meister A, Wobus AM. Primordial germ cell-derived mouse embryonic germ (EG) cells in vitro resemble undifferentiated stem cells with respect to differentiation capacity and cell cycle distribution. Cell Biol Int. 1996;20:579-87.

113 Larsen WJ. The third week. In: Sherman LS, Steven Potter S, Scott WJ, editors. Human embryology. 3 ed. Philadelphia: Churchill Livingstone; 2001. p. 53-78.

114 Power MA, Tam PP. Onset of gastrulation, morphogenesis and somitogenesis in mouse embryos displaying compensatory growth. Anat Embryol (Berl). 1993;187:493-504.

115 Rands GF. Size regulation in the mouse embryo. II. The development of half embryos. J Embryol Exp Morphol. 1986;98:209-17.

116 Janer G, Verhoef A, Gilsing HD, Piersma AH. Use of the rat postimplantation embryo culture to assess the embryotoxic potency within a chemical category and to identify toxic metabolites. Toxicol In vitro. 2008;22:1797-805.

117 Piersma AH, Janer G, Wolterink G, Bessems JG, Hakkert BC, Slob W. Quantitative extrapolation of in vitro whole embryo culture embryotoxicity data to developmental toxicity in vivo using the benchmark dose approach. Toxicol Sci. 2008;101:91-100.

118 Verwei M, van Burgsteden JA, Krul CA, van de Sandt JJ, Freidig AP. Prediction of in vivo embryotoxic effect levels with a combination of in vitro studies and PBPK modelling. Toxicol Lett. 2006; 165:79-87.

119 van der Jagt K, Munn S, Tørsløv J, de Bruijn J. Alternative approaches can reduce the use of test animals under reach. European Commission Report 2004:EUR 21405 EN. 
120 Kultima K, Nystrom AM, Scholz B, Gustafson AL, Dencker L, Stigson M. Valproic acid teratogenicity: a toxicogenomics approach. Environ Health Perspect. 2004;112:1225-35.

121 Fabjan E, Hulzebos E, Mennes W, Piersma AH. A category approach for reproductive effects of phthalates. Crit Rev Toxicol. 2006;36:695-726.

122 Ema M, Kurosaka R, Harazono A, Amano H, Ogawa Y. Phase specificity of developmental toxicity after oral administration of mono-n-butyl phthalate in rats. Arch Environ Contam Toxicol. 1996; 31:170-6.

123 Janssen R, Pennings J, Hodemaekers H, Buisman A, van Oosten M, de Rond L, et al. Host transcription profiles upon primary respiratory syncytial virus infection. J Virol. 2007;81:5958-67.

124 Benjamini Y, Hochberg Y. Controlling the false disovery rate: a practical and powerful approach to multiple testing. J R Stat Soc B. 1995;57:289-300.

125 Rhodes DR, Kalyana-Sundaram S, Tomlins SA, Mahavisno V, Kasper N, Varambally R, et al. Molecular concepts analysis links tumors, pathways, mechanisms, and drugs. Neoplasia. 2007;9:443-54.

126 Smid M, Dorssers LC, Jenster G. Venn Mapping: clustering of heterologous microarray data based on the number of co-occurring differentially expressed genes. Bioinformatics. 2003;19:2065-71.

127 Shannon P, Markiel A, Ozier O, Baliga NS, Wang JT, Ramage D, et al. Cytoscape: a software environment for integrated models of biomolecular interaction networks. Genome Res. 2003;13: 2498-504.

128 Su Al, Wiltshire T, Batalov S, Lapp H, Ching KA, Block D, et al. A gene atlas of the mouse and human protein-encoding transcriptomes. Proc Natl Acad Sci U S A. 2004;101:6062-7.

129 Whitfield ML, Sherlock G, Saldanha AJ, Murray JI, Ball CA, Alexander KE, et al. Identification of genes periodically expressed in the human cell cycle and their expression in tumors. Mol Biol Cell. 2002;13:1977-2000.

130 Bar-Joseph Z, Siegfried Z, Brandeis M, Brors B, Lu Y, Eils R, et al. Genome-wide transcriptional analysis of the human cell cycle identifies genes differentially regulated in normal and cancer cells. Proc Natl Acad Sci U S A. 2008;105:955-60.

131 Zhou Q, Chipperfield H, Melton DA, Wong WH. A gene regulatory network in mouse embryonic stem cells. Proc Natl Acad Sci U S A. 2007;104:16438-43.

132 Behfar A, Perez-Terzic C, Faustino RS, Arrell DK, Hodgson DM, Yamada S, et al. Cardiopoietic programming of embryonic stem cells for tumor-free heart repair. J Exp Med. 2007;204:405-20.

133 Faustino RS, Behfar A, Perez-Terzic C, Terzic A. Genomic chart guiding embryonic stem cell cardiopoiesis. Genome Biol. 2008;9:R6.

134 Alexandrovich A, Arno M, Patient RK, Shah AM, Pizzey JA, Brewer AC. Wnt2 is a direct downstream target of GATA6 during early cardiogenesis. Mech Dev. 2006;123:297-311.

135 Christoforou N, Miller RA, Hill CM, Jie CC, McCallion AS, Gearhart JD. Mouse ES cell-derived cardiac precursor cells are multipotent and facilitate identification of novel cardiac genes. J Clin Invest. 2008; 118:894-903.

136 Wang H, Charles PC, Wu Y, Ren R, Pi X, Moser M, et al. Gene expression profile signatures indicate a role for Wnt signaling in endothelial commitment from embryonic stem cells. Circ Res. 2006;98: 1331-9.

137 Martinez Hoyos J, Fedele M, Battista S, Pentimalli F, Kruhoffer M, Arra C, et al. Identification of the genes up- and down-regulated by the high mobility group A1 (HMGA1) proteins: tissue specificity of the HMGA1-dependent gene regulation. Cancer Res. 2004;64:5728-35.

138 Jelier R, Schuemie MJ, Veldhoven A, Dorssers LC, Jenster G, Kors JA. Anni 2.0: a multipurpose text-mining tool for the life sciences. Genome Biol. 2008;9:R96. 
139 Yang C, Przyborski S, Cooke MJ, Zhang X, Stewart R, Anyfantis G, et al. A key role for telomerase reverse transcriptase unit in modulating human embryonic stem cell proliferation, cell cycle dynamics, and in vitro differentiation. Stem Cells. 2008;26:850-63.

140 Liu N, Feng X, Fang Z, Ma F, Lu S, Lu M, et al. Identification of genes regulated by nanog which is involved in ES cells pluripotency and early differentiation. J Cell Biochem. 2008;104:2348-62.

141 Kim J, Chu J, Shen X, Wang J, Orkin SH. An extended transcriptional network for pluripotency of embryonic stem cells. Cell. 2008;132:1049-61.

142 Masino AM, Gallardo TD, Wilcox CA, Olson EN, Williams RS, Garry DJ. Transcriptional regulation of cardiac progenitor cell populations. Circ Res. 2004;95:389-97.

143 Miller RA, Christoforou N, Pevsner J, McCallion AS, Gearhart JD. Efficient array-based identification of novel cardiac genes through differentiation of mouse ESCs. PLoS ONE. 2008;3:e2176.

144 Synnergren J, Adak S, Englund MC, Giesler TL, Noaksson K, Lindahl A, et al. Cardiomyogenic gene expression profiling of differentiating human embryonic stem cells. J Biotechnol. 2008;134: $162-70$

145 Synnergren J, Akesson K, Dahlenborg K, Vidarsson H, Ameen C, Steel D, et al. Molecular signature of cardiomyocyte clusters derived from human embryonic stem cells. Stem Cells. 2008;26: 1831-40.

146 Kultima K, Fernandez EL, Scholz B, Gustafson AL, Dencker L, Stigson M. Cadmium-induced gene expression changes in the mouse embryo, and the influence of pretreatment with zinc. Reprod Toxicol. 2006;22:636-46.

147 Horie N, Kawamure S, Kohda A. Investigation of 6-aminonicotinamide-induced anomalies and their critical periods in rats. Teratology. 2000;62:42A.

148 Salamon C, Henwood S, Lane C, Miller J, Palazzolo M. Comparison of teratogenic effects of 6-aminonicotinamide in rats when dosed on gestation day 8, 9, or 10. Toxicologist. 1992;12:104.

149 Salamon C, Henwood S, Nokleby J, McKee Pesik P. A comparison of the teratogenic effects of 6-aminonicotinamide in rats and rabbits. Teratology. 1992;45:472.

150 van Dartel DAM, Zeijen NJL, de la Fonteyne LJJ, van Schooten FJ, Piersma AH. Disentangling cellular proliferation and differentiation in the Embryonic Stem cell Test, and its impact on the experimental protocol. Reprod Toxicol. 2009;28:254-61.

151 R. http://www.R-project.org. Last accessed: Dec 2010.

152 Mootha VK, Lindgren CM, Eriksson KF, Subramanian A, Sihag S, Lehar J, et al. PGC-1alpha-responsive genes involved in oxidative phosphorylation are coordinately downregulated in human diabetes. Nat Genet. 2003;34:267-73.

153 Sharov AA, Piao Y, Matoba R, Dudekula DB, Qian Y, VanBuren V, et al. Transcriptome analysis of mouse stem cells and early embryos. PLoS Biol. 2003;1:E74.

154 Doss MX, Chen S, Winkler J, Hippler-Altenburg R, Odenthal M, Wickenhauser C, et al. Transcriptomic and phenotypic analysis of murine embryonic stem cell derived BMP2+ lineage cells: an insight into mesodermal patterning. Genome Biol. 2007;8:R184.

155 Boyer LA, Lee TI, Cole MF, Johnstone SE, Levine SS, Zucker JP, et al. Core transcriptional regulatory circuitry in human embryonic stem cells. Cell. 2005;122:947-56.

156 Ivanova N, Dobrin R, Lu R, Kotenko I, Levorse J, DeCoste C, et al. Dissecting self-renewal in stem cells with RNA interference. Nature. 2006;442:533-8.

157 Sharov AA, Masui S, Sharova LV, Piao Y, Aiba K, Matoba R, et al. Identification of Pou5f1, Sox2, and Nanog downstream target genes with statistical confidence by applying a novel algorithm to time course microarray and genome-wide chromatin immunoprecipitation data. BMC Genomics. 2008;9:269. 
158 Assou S, Le Carrour T, Tondeur S, Strom S, Gabelle A, Marty S, et al. A meta-analysis of human embryonic stem cells transcriptome integrated into a web-based expression atlas. Stem Cells. 2007;25:961-73.

159 Bhattacharya B, Miura T, Brandenberger R, Mejido J, Luo Y, Yang AX, et al. Gene expression in human embryonic stem cell lines: unique molecular signature. Blood. 2004;103:2956-64.

160 Sperger JM, Chen X, Draper JS, Antosiewicz JE, Chon CH, Jones SB, et al. Gene expression patterns in human embryonic stem cells and human pluripotent germ cell tumors. Proc Natl Acad Sci U S A. 2003;100:13350-5.

161 Glover $\mathrm{CH}$, Marin M, Eaves CJ, Helgason CD, Piret JM, Bryan J. Meta-analysis of differentiating mouse embryonic stem cell gene expression kinetics reveals early change of a small gene set. PLoS Comput Biol. 2006;2:e158.

162 Palmqvist L, Glover CH, Hsu L, Lu M, Bossen B, Piret JM, et al. Correlation of murine embryonic stem cell gene expression profiles with functional measures of pluripotency. Stem Cells. 2005;23: 663-80.

163 Brandenberger R, Wei H, Zhang S, Lei S, Murage J, Fisk GJ, et al. Transcriptome characterization elucidates signaling networks that control human ES cell growth and differentiation. Nat Biotechnol. 2004;22:707-16.

164 Van Hoof D, Passier R, Ward-Van Oostwaard D, Pinkse MW, Heck AJ, Mummery CL, et al. A quest for human and mouse embryonic stem cell-specific proteins. Mol Cell Proteomics. 2006;5:1261-73.

165 Gu G, Wells JM, Dombkowski D, Preffer F, Aronow B, Melton DA. Global expression analysis of gene regulatory pathways during endocrine pancreatic development. Development. 2004;131:165-79.

166 Karsten SL, Kudo LC, Jackson R, Sabatti C, Kornblum HI, Geschwind DH. Global analysis of gene expression in neural progenitors reveals specific cell-cycle, signaling, and metabolic networks. Dev Biol. 2003;261:165-82.

167 Steinberg MS. Differential adhesion in morphogenesis: a modern view. Curr Opin Genet Dev. 2007;17:281-6.

168 van de Waterbeemd B, Streefland M, Pennings JLA, van der Pol L, Beuvery C, Tramper J, et al. Gene-expression-based quality scores indicate optimal harvest point in Bordetella pertussis cultivation for vaccine production. Biotechnol Bioeng. 2009;103:900-8.

169 Liu Z, Wang M, Alvarez JV, Bonney ME, Chen CC, D'Cruz C, et al. Singular value decompositionbased regression identifies activation of endogenous signaling pathways in vivo. Genome Biol. 2008;9:R180.

170 Marx-Stoelting P, Adriaens E, Ahr HJ, Bremer S, Garthoff B, Gelbke HP, et al. A review of the implementation of the Embryonic Stem cell Test (EST). The report and recommendations of an ECVAM/ ReProTect Workshop. Altern Lab Anim. 2009;37:313-28.

171 Larsen WJ. Human embryology. 3 ed. Philadelphia: Churchill Livingstone; 2001.

172 Baker TK, Carfagna MA, Gao H, Dow ER, Li Q, Searfoss GH, et al. Temporal gene expression analysis of monolayer cultured rat hepatocytes. Chem Res Toxicol. 2001;14:1218-31.

173 Beigel J, Fella K, Kramer PJ, Kroeger M, Hewitt P. Genomics and proteomics analysis of cultured primary rat hepatocytes. Toxicol In vitro. 2008;22:171-81.

174 Baken KA, van Loveren H, Pennings JL, de Vries A, Breit TM, van Steeg H. Gene expression profiling of Bis(tri-n-butyltin)oxide (TBTO)-induced immunotoxicity in mice and rats. J Immunotoxicol. 2006;3:227-44.

175 Hamadeh HK, Bushel PR, Jayadev S, Martin K, DiSorbo O, Sieber S, et al. Gene expression analysis reveals chemical-specific profiles. Toxicol Sci. 2002;67:219-31. 
176 Waring JF, Ciurlionis R, Jolly RA, Heindel M, Ulrich RG. Microarray analysis of hepatotoxins in vitro reveals a correlation between gene expression profiles and mechanisms of toxicity. Toxicol Lett. 2001;120:359-68.

177 van Dartel DAM, Pennings JLA, van Schooten FJ, Piersma AH. Transcriptomics-based identification of developmental toxicants through their interference with cardiomyocyte differentiation of embryonic stem cells. Toxicol Appl Pharmacol. 2010;243:420-8.

178 O'Brien J, Wilson I, Orton T, Pognan F. Investigation of the Alamar Blue (resazurin) fluorescent dye for the assessment of mammalian cell cytotoxicity. Eur J Biochem. 2000;267:5421-6.

179 Irizarry RA, Hobbs B, Collin F, Beazer-Barclay YD, Antonellis KJ, Scherf U, et al. Exploration, normalization, and summaries of high density oligonucleotide array probe level data. Biostatistics. 2003; 4:249-64.

180 Bolstad BM, Irizarry RA, Astrand M, Speed TP. A comparison of normalization methods for high density oligonucleotide array data based on variance and bias. Bioinformatics. 2003;19:185-93.

181 de Leeuw WC, Rauwerda H, Jonker MJ, Breit TM. Salvaging Affymetrix probes after probe-level re-annotation. BMC Res Notes. 2008;1:66.

182 Dai M, Wang P, Boyd AD, Kostov G, Athey B, Jones EG, et al. Evolving gene/transcript definitions significantly alter the interpretation of GeneChip data. Nucleic Acids Res. 2005;33:e175.

183 Huang da W, Sherman BT, Tan Q, Kir J, Liu D, Bryant D, et al. DAVID Bioinformatics Resources: expanded annotation database and novel algorithms to better extract biology from large gene lists. Nucleic Acids Res. 2007;35:W169-75.

184 Stedman DB, Welsch F. Inhibition of DNA synthesis in mouse whole embryo culture by 2-methoxyacetic acid and attenuation of the effects by simple physiological compounds. Toxicol Lett. 1989;45:111-7.

185 Rasjad C, Yamashita K, Datu AR, Yasuda M. Pathogenesis of limb malformations in mice induced by methoxyacetic acid. Hiroshima J Med Sci. 1991;40:101-7.

186 Cotariu D, Zaidman JL. Developmental toxicity of valproic acid. Life Sci. 1991;48:1341-50.

187 Durston AJ, Timmermans JP, Hage WJ, Hendriks HF, de Vries NJ, Heideveld M, et al. Retinoic acid causes an anteroposterior transformation in the developing central nervous system. Nature. 1989;340:140-4.

188 Rutledge JC, Shourbaji AG, Hughes LA, Polifka JE, Cruz YP, Bishop JB, et al. Limb and lower-body duplications induced by retinoic acid in mice. Proc Natl Acad Sci U S A. 1994;91:5436-40.

189 Andersen ME, Clewell HJ, 3rd, Bermudez E, Willson GA, Thomas RS. Genomic signatures and dosedependent transitions in nasal epithelial responses to inhaled formaldehyde in the rat. Toxicol Sci. 2008;105:368-83.

190 Auerbach SS, Shah RR, Mav D, Smith CS, Walker NJ, Vallant MK, et al. Predicting the hepatocarcinogenic potential of alkenylbenzene flavoring agents using toxicogenomics and machine learning. Toxicol Appl Pharmacol. 2010;243:300-14.

191 Jonker MJ, Bruning O, van Iterson M, Schaap MM, van der Hoeven TV, Vrieling $H$, et al. Finding transcriptomics biomarkers for in vivo identification of (non-)genotoxic carcinogens using wildtype and Xpa/p53 mutant mouse models. Carcinogenesis. 2009;30:1805-12.

192 NRC. Toxicity testing in the 21 st century. A vision and a strategy. Washington, DC: The national academies press; 2007.

193 Chapin RE, Stedman DB. Endless possibilities: stem cells and the vision for toxicology testing in the 21 st century. Toxicol Sci. 2009;112:17-22. 
194 van Dartel DAM, Pennings JLA, de la Fonteyne LJJ, van Herwijnen M, van Delft JH, van Schooten FJ, et al. Monitoring developmental toxicity in the Embryonic Stem cell Test using differential gene expression of differentiation-related genes. Toxicol Sci. 2010;116:130-9.

Aiba K, Sharov AA, Carter MG, Foroni C, Vescovi AL, Ko MS. Defining a developmental path to neural fate by global expression profiling of mouse embryonic stem cells and adult neural stem/ progenitor cells. Stem Cells. 2006;24:889-95.

196 de Jong E, Louisse J, Verwei M, Blaauboer BJ, van de Sandt JJ, Woutersen RA, et al. Relative developmental toxicity of glycol ether alkoxy acid metabolites in the Embryonic Stem cell Test as compared with the in vivo potency of their parent compounds. Toxicol Sci. 2009;110:117-24.

197 Genschow E, Seiler A, Spielmann H. Considering the test performance for three class data using linear discriminant analysis: a case study. Altern Lab Anim. 2004;Supplement 1:713-23.

198 Mylchreest E, Cattley RC, Foster PM. Male reproductive tract malformations in rats following gestational and lactational exposure to Di(n-butyl) phthalate: an antiandrogenic mechanism? Toxicol Sci. 1998;43:47-60.

199 Yagi Y, Nakamura Y, Tomita I, Tsuchikawa K, Shimoi N. Teratogenic potential of di- and mono-(2ethylhexyl)phthalate in mice. J Environ Pathol Toxicol. 1980;4:533-44.

200 Kavlock R, Boekelheide K, Chapin R, Cunningham M, Faustman E, Foster P, et al. NTP Center for the Evaluation of Risks to Human Reproduction: phthalates expert panel report on the reproductive and developmental toxicity of di-n-octyl phthalate. Reprod Toxicol. 2002;16:721-34.

201 Knudsen TB, Martin MT, Kavlock RJ, Judson RS, Dix DJ, Singh AV. Profiling the activity of environmental chemicals in prenatal developmental toxicity studies using the U.S. EPA's ToxRefDB. Reprod Toxicol. 2009;28:209-19.

202 Finnell RH, MohI VK, Bennett GD, Taylor SM. Failure of epoxide formation to influence carbamazepine-induced teratogenesis in a mouse model. Teratog Carcinog Mutagen. 1986;6:393-401.

203 Jones KL, Lacro RV, Johnson KA, Adams J. Pattern of malformations in the children of women treated with carbamazepine during pregnancy. N Engl J Med. 1989;320:1661-6.

204 Elsner J, Hodel B, Suter KE, Oelke D, Ulbrich B, Schreiner G, et al. Detection limits of different approaches in behavioral teratology, and correlation of effects with neurochemical parameters. Neurotoxicol Teratol. 1988;10:155-67.

205 Kutscher CL, Sembrat M, Kutscher CS, Kutscher NL. Effects of the high methylmercury dose used in the collaborative behavioral teratology study on brain anatomy. Neurobehav Toxicol Teratol. 1985;7:775-7.

206 Howe AM, Webster WS. Exposure of the pregnant rat to warfarin and vitamin K1: an animal model of intraventricular hemorrhage in the fetus. Teratology. 1990;42:413-20.

207 Kang YJ, Zolna L, Manson JM. Strain differences in response of Sprague-Dawley and Long Evans Hooded rats to the teratogen nitrofen. Teratology. 1986;34:213-23.

208 Jordan RL, Wilson JG, Schumacher HJ. Embryotoxicity of the folate antagonist methotrexate in rats and rabbits. Teratology. 1977;15:73-9.

209 Schena M, Shalon D, Davis RW, Brown PO. Quantitative monitoring of gene expression patterns with a complementary DNA microarray. Science. 1995;270:467-70.

210 Uehara T, Hirode M, Ono A, Kiyosawa N, Omura K, Shimizu T, et al. A toxicogenomics approach for early assessment of potential non-genotoxic hepatocarcinogenicity of chemicals in rats. Toxicology. 2008;250:15-26.

211 Ezendam J, Staedtler F, Pennings J, Vandebriel RJ, Pieters R, Harleman JH, et al. Toxicogenomics of subchronic hexachlorobenzene exposure in Brown Norway rats. Environ Health Perspect. 2004; 112:782-91. 
212 Naciff JM, Khambatta ZS, Reichling TD, Carr GJ, Tiesman JP, Singleton DW, et al. The genomic response of Ishikawa cells to bisphenol A exposure is dose- and time-dependent. Toxicology. 2010;270:137-49.

213 Robinson JF, Guerrette Z, Yu X, Hong S, Faustman EM. A systems-based approach to investigate dose- and time-dependent methylmercury-induced gene expression response in C57BL/6 mouse embryos undergoing neurulation. Birth Defects Res B Dev Reprod Toxicol. 2010;89:188-200.

214 van Dartel DAM, Pennings JLA, de la Fonteyne LJJ, Brauers KJJ, Claessen SMH, van Delft JH, et al. Evaluation of developmental toxicant identification using gene expression profiling in embryonic stem cell differentiation cultures. Toxicol Sci. 2011;119:126-34.

215 Farag AT, Ibrahim HH. Developmental toxic effects of antifungal flusilazole administered by gavage to mice. Birth Defects Res B Dev Reprod Toxicol. 2007;80:12-7.

216 Zarn JA, Bruschweiler BJ, Schlatter JR. Azole fungicides affect mammalian steroidogenesis by inhibiting sterol 14 alpha-demethylase and aromatase. Environ Health Perspect. 2003;111:255-61.

217 Doniger SW, Salomonis N, Dahlquist KD, Vranizan K, Lawlor SC, Conklin BR. MAPPFinder: using Gene Ontology and GenMAPP to create a global gene-expression profile from microarray data. Genome Biol. 2003;4:R7.

218 van Delft JH, van Agen E, van Breda SG, Herwijnen MH, Staal YC, Kleinjans JC. Discrimination of genotoxic from non-genotoxic carcinogens by gene expression profiling. Carcinogenesis. 2004; 25:1265-76.

219 Groebe K, Hayess K, Klemm-Manns M, Schwall G, Wozny W, Steemans M, et al. Unexpected common mechanistic pathways for embryotoxicity of warfarin and lovastatin. Reprod Toxicol. 2010; 30:121-30.

220 Kultima K, Jergil M, Salter H, Gustafson AL, Dencker L, Stigson M. Early transcriptional responses in mouse embryos as a basis for selection of molecular markers predictive of valproic acid teratogenicity. Reprod Toxicol. 2010;30:457-68.

221 Singh AV, Knudsen KB, Knudsen TB. Computational systems analysis of developmental toxicity: design, development and implementation of a Birth Defects Systems Manager (BDSM). Reprod Toxicol. 2005;19:421-39.

222 Liguori GL, Borges AC, D'Andrea D, Liguoro A, Goncalves L, Salgueiro AM, et al. Cripto-independent Nodal signaling promotes positioning of the A-P axis in the early mouse embryo. Dev Biol. 2008; 315:280-9.

223 Puelles E, Annino A, Tuorto F, Usiello A, Acampora D, Czerny T, et al. Otx2 regulates the extent, identity and fate of neuronal progenitor domains in the ventral midbrain. Development. 2004; 131:2037-48.

224 Oginuma M, Hirata T, Saga Y. Identification of presomitic mesoderm (PSM)-specific Mesp1 enhancer and generation of a PSM-specific Mesp1/Mesp2-null mouse using BAC-based rescue technology. Mech Dev. 2008;125:432-40.

225 Heinloth AN, Irwin RD, Boorman GA, Nettesheim P, Fannin RD, Sieber SO, et al. Gene expression profiling of rat livers reveals indicators of potential adverse effects. Toxicol Sci. 2004;80:193-202.

226 Ghannoum MA, Rice LB. Antifungal agents: mode of action, mechanisms of resistance, and correlation of these mechanisms with bacterial resistance. Clin Microbiol Rev. 1999;12:501-17.

227 Vanden Bossche H, Marichal P, Gorrens J, Coene MC. Biochemical basis for the activity and selectivity of oral antifungal drugs. Br J Clin Pract Suppl. 1990;71:41-6.

228 Trzaskos JM, Henry MJ. Comparative effects of the azole-based fungicide flusilazole on yeast and mammalian lanosterol 14 alpha-methyl demethylase. Antimicrob Agents Chemother. 1989;33: 1228-31. 
229 Tully DB, Bao W, Goetz AK, Blystone CR, Ren H, Schmid JE, et al. Gene expression profiling in liver and testis of rats to characterize the toxicity of triazole fungicides. Toxicol Appl Pharmacol. 2006; 215:260-73.

230 De Haan JR, Piek E, van Schaik RC, de Vlieg J, Bauerschmidt S, Buydens LM, et al. Integrating gene expression and GO classification for PCA by preclustering. BMC Bioinformatics. 2010;11:158.

231 Aiba K, Nedorezov T, Piao Y, Nishiyama A, Matoba R, Sharova LV, et al. Defining developmental potency and cell lineage trajectories by expression profiling of differentiating mouse embryonic stem cells. DNA Res. 2009;16:73-80.

232 Fang H, Yang Y, Li C, Fu S, Yang Z, Jin G, et al. Transcriptome analysis of early organogenesis in human embryos. Dev Cell. 2010;19:174-84.

233 Rodenburg W, Heidema AG, Boer JM, Bovee-Oudenhoven IM, Feskens EJ, Mariman EC, et al. A framework to identify physiological responses in microarray-based gene expression studies: selection and interpretation of biologically relevant genes. Physiol Genomics. 2008;33:78-90.

234 Lilienblum W, Dekant W, Foth H, Gebel T, Hengstler JG, Kahl R, et al. Alternative methods to safety studies in experimental animals: role in the risk assessment of chemicals under the new European Chemicals Legislation (REACH). Arch Toxicol. 2008;82:211-36.

235 van Dartel DAM, Pennings JLA, de la Fonteyne LJJ, Brauers KJJ, Claessen SMH, van Delft JH, et al. Concentration-dependent gene expression responses to flusilazole in embryonic stem cell differentiation cultures. Toxicol Appl Pharmacol. 2011;251:110-8.

236 Fliri AF, Loging WT, Thadeio PF, Volkmann RA. Biological spectra analysis: Linking biological activity profiles to molecular structure. Proc Natl Acad Sci U S A. 2005;102:261-6.

237 Melnick JS, Janes J, Kim S, Chang JY, Sipes DG, Gunderson D, et al. An efficient rapid system for profiling the cellular activities of molecular libraries. Proc Natl Acad Sci U S A. 2006;103:3153-8.

238 Martin MT, Brennan RJ, Hu W, Ayanoglu E, Lau C, Ren H, et al. Toxicogenomic study of triazole fungicides and perfluoroalkyl acids in rat livers predicts toxicity and categorizes chemicals based on mechanisms of toxicity. Toxicol Sci. 2007;97:595-613.

239 Steiner G, Suter L, Boess F, Gasser R, de Vera MC, Albertini S, et al. Discriminating different classes of toxicants by transcript profiling. Environ Health Perspect. 2004;112:1236-48.

240 Daston GP, Naciff JM. Predicting developmental toxicity through toxicogenomics. Birth Defects Res C Embryo Today. 2010;90:110-7.

241 Liu K, Lehmann KP, Sar M, Young SS, Gaido KW. Gene expression profiling following in utero exposure to phthalate esters reveals new gene targets in the etiology of testicular dysgenesis. Biol Reprod. 2005;73:180-92.

242 Lamb J, Crawford ED, Peck D, Modell JW, Blat IC, Wrobel MJ, et al. The Connectivity Map: using gene-expression signatures to connect small molecules, genes, and disease. Science. 2006;313: 1929-35.

243 Menegola E, Broccia ML, Di Renzo F, Giavini E. Pathogenic pathways in fluconazole-induced branchial arch malformations. Birth Defects Res A Clin Mol Teratol. 2003;67:116-24.

244 Tiboni GM, Marotta F, Carletti E. Fluconazole alters CYP26 gene expression in mouse embryos. Reprod Toxicol. 2009;27:199-202.

245 Rusyn I, Peters JM, Cunningham ML. Modes of action and species-specific effects of di-(2ethylhexyl)phthalate in the liver. Crit Rev Toxicol. 2006;36:459-79.

$246 \mathrm{Nau} \mathrm{H}$, Scott WJ, Jr. Weak acids may act as teratogens by accumulating in the basic milieu of the early mammalian embryo. Nature. 1986;323:276-8.

247 Carbon S, Ireland A, Mungall CJ, Shu S, Marshall B, Lewis S. AmiGO: online access to ontology and annotation data. Bioinformatics. 2009;25:288-9. 
248 Schoonen WG, Kloks CP, Ploemen JP, Smit MJ, Zandberg P, Horbach GJ, et al. Uniform procedure of (1)H NMR analysis of rat urine and toxicometabonomics Part II: comparison of NMR profiles for classification of hepatotoxicity. Toxicol Sci. 2007;98:286-97.

249 Marotta F, Tiboni GM. Molecular aspects of azoles-induced teratogenesis. Expert Opin Drug Metab Toxicol. 2010;6:461-82.

250 Rohwedel J, Guan K, Wobus AM. Induction of cellular differentiation by retinoic acid in vitro. Cells Tissues Organs. 1999;165:190-202.

251 Robinson JF, Yu X, Moreira EG, Hong S, Faustman EM. Arsenic- and cadmium-induced toxicogenomic response in mouse embryos undergoing neurulation. Toxicol Appl Pharmacol. 2011;250: 117-29.

252 Natsoulis G, El Ghaoui L, Lanckriet GR, Tolley AM, Leroy F, Dunlea S, et al. Classification of a large microarray data set: algorithm comparison and analysis of drug signatures. Genome Res. 2005;15: 724-36.

253 Martin MT, Judson RS, Reif DM, Kavlock RJ, Dix DJ. Profiling chemicals based on chronic toxicity results from the U.S. EPA ToxRef Database. Environ Health Perspect. 2009;117:392-9.

254 Piersma AH, van Dartel DAM, van der Ven LTM. Alternative Methods in Developmental Toxicology. In: Knudsen TB, Daston GP, editors. Comprehensive Toxicology: Developmental toxicology. New York: Elsevier; 2010. p. 293-307.

255 Buesen R, Genschow E, Slawik B, Visan A, Spielmann H, Luch A, et al. Embryonic stem cell test remastered: comparison between the validated EST and the new molecular FACS-EST for assessing developmental toxicity in vitro. Toxicol Sci. 2009;108:389-400.

256 Osman AM, van Dartel DAM, Zwart E, Blokland M, Pennings JLA, Piersma AH. Proteome profiling of mouse embryonic stem cells to define markers for cell differentiation and embryotoxicity. Reprod Toxicol. 2010;30:322-32.

257 Huang da W, Sherman BT, Lempicki RA. Bioinformatics enrichment tools: paths toward the comprehensive functional analysis of large gene lists. Nucleic Acids Res. 2009;37:1-13.

258 Pennings JLA, van Dartel DAM, Pronk TE, Hendriksen PJ, Piersma AH. Identification by gene coregulation mapping of novel genes involved in embryonic stem cell differentiation. Stem Cells Dev. 2011;20:115-26.

259 Jensen LJ, Kuhn M, Stark M, Chaffron S, Creevey C, Muller J, et al. STRING 8--a global view on proteins and their functional interactions in 630 organisms. Nucleic Acids Res. 2009;37:D412-6.

260 U.S.E.P.A. An approach to using toxicogenomic data in US EPA human health risk assessments: a dibutyl phthalate case study. EPA/600/R-09/028f. 2009.

261 Breiman L. Random forest. Machine learning. 2001;45:5-32.

262 Buesen R, Visan A, Genschow E, Slawik B, Spielmann H, Seiler A. Trends in improving the Embryonic Stem cell Test (EST): an overview. Altex. 2004;21:15-22.

263 Chang CW, Tsai CW, Wang HF, Tsai HC, Chen HY, Tsai TF, et al. Identification of a developmentally regulated striatum-enriched zinc-finger gene, Nolz-1, in the mammalian brain. Proc Natl Acad Sci U S A. 2004;101:2613-8.

264 Miyamoto S, Hidaka K, Jin D, Morisaki T. RNA-binding proteins Rbm38 and Rbm24 regulate myogenic differentiation via p21-dependent and -independent regulatory pathways. Genes Cells. 2009;14:1241-52.

265 Nelson TJ, Faustino RS, Chiriac A, Crespo-Diaz R, Behfar A, Terzic A. CXCR4+/FLK-1+ biomarkers select a cardiopoietic lineage from embryonic stem cells. Stem Cells. 2008;26:1464-73. 
266 Ganter B, Tugendreich S, Pearson Cl, Ayanoglu E, Baumhueter S, Bostian KA, et al. Development of a large-scale chemogenomics database to improve drug candidate selection and to understand mechanisms of chemical toxicity and action. J Biotechnol. 2005;119:219-44.

267 Segal E, Friedman N, Koller D, Regev A. A module map showing conditional activity of expression modules in cancer. Nat Genet. 2004;36:1090-8.

268 Tseng GC, Cheng C, Yu YP, Nelson J, Michalopoulos G, Luo JH. Investigating Multi-cancer Biomarkers and Their Cross-predictability in the Expression Profiles of Multiple Cancer Types. Biomark Insights. 2009;4:57-79.

269 Yang Y, Pospisil P, lyer LK, Adelstein SJ, Kassis Al. Integrative genomic data mining for discovery of potential blood-borne biomarkers for early diagnosis of cancer. PLoS One. 2008;3:e3661.

270 Jenner RG, Young RA. Insights into host responses against pathogens from transcriptional profiling. Nat Rev Microbiol. 2005;3:281-94.

271 Pennings JL, Kimman TG, Janssen R. Identification of a common gene expression response in different lung inflammatory diseases in rodents and macaques. PLoS One. 2008;3:e2596.

272 de Magalhaes JP, Curado J, Church GM. Meta-analysis of age-related gene expression profiles identifies common signatures of aging. Bioinformatics. 2009;25:875-81.

273 Rodriguez-Zas SL, Ko Y, Adams HA, Southey BR. Advancing the understanding of the embryo transcriptome co-regulation using meta-, functional, and gene network analysis tools. Reproduction. 2008;135:213-24.

274 Stuart JM, Segal E, Koller D, Kim SK. A gene-coexpression network for global discovery of conserved genetic modules. Science. 2003;302:249-55.

275 Luo F, Yang Y, Zhong J, Gao H, Khan L, Thompson DK, et al. Constructing gene co-expression networks and predicting functions of unknown genes by random matrix theory. BMC Bioinformatics. 2007;8:299.

276 Presson AP, Sobel EM, Papp JC, Suarez CJ, Whistler T, Rajeevan MS, et al. Integrated weighted gene co-expression network analysis with an application to chronic fatigue syndrome. BMC Syst Biol. 2008;2:95.

277 Wang K, Narayanan M, Zhong H, Tompa M, Schadt EE, Zhu J. Meta-analysis of inter-species liver co-expression networks elucidates traits associated with common human diseases. PLoS Comput Biol. 2009;5:e1000616.

278 Gustin MP, Paultre CZ, Randon J, Bricca G, Cerutti C. Functional meta-analysis of double connectivity in gene coexpression networks in mammals. Physiol Genomics. 2008;34:34-41.

279 Fishman MC, Chien KR. Fashioning the vertebrate heart: earliest embryonic decisions. Development. 1997;124:2099-117.

280 Srivastava D, Olson EN. A genetic blueprint for cardiac development. Nature. 2000;407:221-6.

281 Beqqali A, van Eldik W, Mummery C, Passier R. Human stem cells as a model for cardiac differentiation and disease. Cell Mol Life Sci. 2009;66:800-13.

282 Bader GD, Hogue CW. An automated method for finding molecular complexes in large protein interaction networks. BMC Bioinformatics. 2003;4:2.

283 Maere S, Heymans K, Kuiper M. BiNGO: a Cytoscape plugin to assess overrepresentation of gene ontology categories in biological networks. Bioinformatics. 2005;21:3448-9.

284 Larsson O, Wennmalm K, Sandberg R. Comparative microarray analysis. OMICS. 2006;10:381-97.

285 Alako BT, Veldhoven A, van Baal S, Jelier R, Verhoeven S, Rullmann T, et al. CoPub Mapper: mining MEDLINE based on search term co-publication. BMC Bioinformatics. 2005;6:51.

286 Frijters R, Heupers B, van Beek P, Bouwhuis M, van Schaik R, de Vlieg J, et al. CoPub: a literaturebased keyword enrichment tool for microarray data analysis. Nucleic Acids Res. 2008;36:W406-10. 
287 Rubinstein R, Simon I. MILANO--custom annotation of microarray results using automatic literature searches. BMC Bioinformatics. 2005;6:12.

288 Wren JD. A global meta-analysis of microarray expression data to predict unknown gene functions and estimate the literature-data divide. Bioinformatics. 2009;25:1694-701.

289 Cahan P, Ahmad AM, Burke H, Fu S, Lai Y, Florea L, et al. List of lists-annotated (LOLA): a database for annotation and comparison of published microarray gene lists. Gene. 2005;360:78-82.

290 Finocchiaro G, Mancuso F, Muller H. Mining published lists of cancer related microarray experiments: identification of a gene expression signature having a critical role in cell-cycle control. BMC Bioinformatics. 2005;6 Suppl 4:S14.

291 Newman JC, Weiner AM. L2L: a simple tool for discovering the hidden significance in microarray expression data. Genome Biol. 2005;6:R81.

292 Pinz I, Robbins J, Rajasekaran NS, Benjamin IJ, Ingwall JS. Unmasking different mechanical and energetic roles for the small heat shock proteins CryAB and HSPB2 using genetically modified mouse hearts. FASEB J. 2008;22:84-92.

293 Sato Y, Probst HC, Tatsumi R, Ikeuchi Y, Neuberger MS, Rada C. Deficiency in APOBEC2 leads to a shift in muscle fiber type, diminished body mass, and myopathy. J Biol Chem. 2010;285:7111-8.

294 Yang G, Wu L, Bryan S, Khaper N, Mani S, Wang R. Cystathionine gamma-lyase deficiency and overproliferation of smooth muscle cells. Cardiovasc Res. 2010;86:487-95.

295 Degousee N, Fazel S, Angoulvant D, Stefanski E, Pawelzik SC, Korotkova M, et al. Microsomal prostaglandin E2 synthase-1 deletion leads to adverse left ventricular remodeling after myocardial infarction. Circulation. 2008;117:1701-10.

296 Chang L, Zhang J, Tseng YH, Xie CQ, llany J, Bruning JC, et al. Rad GTPase deficiency leads to cardiac hypertrophy. Circulation. 2007;116:2976-83.

297 Li L, Baibakov B, Dean J. A subcortical maternal complex essential for preimplantation mouse embryogenesis. Dev Cell. 2008;15:416-25.

298 Li X, Ito M, Zhou F, Youngson N, Zuo X, Leder P, et al. A maternal-zygotic effect gene, Zfp57, maintains both maternal and paternal imprints. Dev Cell. 2008;15:547-57.

299 Lattin JE, Schroder K, Su Al, Walker JR, Zhang J, Wiltshire T, et al. Expression analysis of G ProteinCoupled Receptors in mouse macrophages. Immunome Res. 2008;4:5.

300 Wu C, Orozco C, Boyer J, Leglise M, Goodale J, Batalov S, et al. BioGPS: an extensible and customizable portal for querying and organizing gene annotation resources. Genome Biol. 2009;10:R130.

301 Dvash T, Mayshar Y, Darr H, McElhaney M, Barker D, Yanuka O, et al. Temporal gene expression during differentiation of human embryonic stem cells and embryoid bodies. Hum Reprod. 2004; 19:2875-83.

302 Cao F, Wagner RA, Wilson KD, Xie X, Fu JD, Drukker M, et al. Transcriptional and functional profiling of human embryonic stem cell-derived cardiomyocytes. PLoS ONE. 2008;3:e3474.

303 Potta SP, Liang H, Pfannkuche K, Winkler J, Chen S, Doss MX, et al. Functional Characterization and Transcriptome Analysis of Embryonic Stem Cell-Derived Contractile Smooth Muscle Cells. Hypertension. 2008;53:196-204.

304 Rolletschek A, Schroeder IS, Schulz H, Hummel O, Huebner N, Wobus AM. Characterization of mouse embryonic stem cell differentiation into the pancreatic lineage in vitro by transcriptional profiling, quantitative RT-PCR and immunocytochemistry. Int J Dev Biol. 2010;54:41-54.

305 Abranches E, Silva M, Pradier L, Schulz H, Hummel O, Henrique D, et al. Neural differentiation of embryonic stem cells in vitro: a road map to neurogenesis in the embryo. PLoS One. 2009;4:e6286. 
306 zur Nieden NI, Price FD, Davis LA, Everitt RE, Rancourt DE. Gene profiling on mixed embryonic stem cell populations reveals a biphasic role for beta-catenin in osteogenic differentiation. Mol Endocrinol. 2007;21:674-85.

307 Seewald MJ, Ellinghaus P, Kassner A, Stork I, Barg M, Niebrugge S, et al. Genomic profiling of developing cardiomyocytes from recombinant murine embryonic stem cells reveals regulation of transcription factor clusters. Physiol Genomics. 2009;38:7-15.

308 Hermsen SAB, Pronk TE, van den Brandhof EJ, van der Ven LTM, Piersma AH. Class specific gene expression changes in the zebrafish embryo after exposure to glycol ether alkoxy acids and 1,2,4-triazole antifungals. Submitted. 2011.

309 Kienhuis AS, Bessems JG, Pennings JL, Driessen M, Luijten M, van Delft JH, et al. Application of toxicogenomics in hepatic systems toxicology for risk assessment: Acetaminophen as a case study. Toxicol Appl Pharmacol. 2011;250:96-107.

310 Van den Berg M, Birnbaum L, Bosveld AT, Brunstrom B, Cook P, Feeley M, et al. Toxic equivalency factors (TEFs) for PCBs, PCDDs, PCDFs for humans and wildlife. Environ Health Perspect. 1998;106: 775-92.

311 Peters AK, Steemans M, Hansen E, Mesens N, Verheyen GR, Vanparys P. Evaluation of the embryotoxic potency of compounds in a newly revised high throughput Embryonic Stem cell Test. Toxicol Sci. 2008;105:342-50.

312 de Jong E, Doedée AMCM, Reis-Fernandesc MA, Nau H, Piersma AH. Potency ranking of valproic acid analogues as to inhibition of cardiac differentiation of embryonic stem cells in comparison to their in vivo embryotoxicity. Toxicol Sci. 2011;doi:10.1016/j.reprotox.2010.11.012

313 Hurtt ME, Cappon GD, Browning A. Proposal for a tiered approach to developmental toxicity testing for veterinary pharmaceutical products for food-producing animals. Food Chem Toxicol. 2003;41:611-9.

314 Thomson JA, Itskovitz-Eldor J, Shapiro SS, Waknitz MA, Swiergiel JJ, Marshall VS, et al. Embryonic stem cell lines derived from human blastocysts. Science. 1998;282:1145-7.

315 Mei Y, Saha K, Bogatyrev SR, Yang J, Hook AL, Kalcioglu ZI, et al. Combinatorial development of biomaterials for clonal growth of human pluripotent stem cells. Nat Mater. 2010;9:768-78.

316 Blaauboer BJ. Biokinetic modeling and in vitro-in vivo extrapolations. J Toxicol Environ Health B Crit Rev. 2010;13:242-52.

317 De Buck SS, Mackie CE. Physiologically based approaches towards the prediction of pharmacokinetics: in vitro-in vivo extrapolation. Expert Opin Drug Metab Toxicol. 2007;3:865-78.

318 Louisse J, de Jong E, van de Sandt JJ, Blaauboer BJ, Woutersen RA, Piersma AH, et al. The use of in vitro toxicity data and physiologically based kinetic modeling to predict dose-response curves for in vivo developmental toxicity of glycol ethers in rat and man. Toxicol Sci. 2010;118:470-84.

319 Jelier R, Jenster G, Dorssers LC, Wouters BJ, Hendriksen PJ, Mons B, et al. Text-derived concept profiles support assessment of DNA microarray data for acute myeloid leukemia and for androgen receptor stimulation. BMC Bioinformatics. 2007;8:14.

320 Knudsen KB, Singh AV, Knudsen TB. Data input module for Birth Defects Systems Manager. Reprod Toxicol. 2005;20:369-75.

321 Singh AV, Kavlock RJ, Richard AM, C. Y. Computational toxicology. In: Knudsen TB, Daston GP, editors. Developmental Toxicology. Kidlington: Elsevier; 2010. p. 307-38.

322 Spielmann H, Seiler A, Bremer S, Hareng L, Hartung T, Ahr H, et al. The Practical Application of Three Validated In vitro Embryotoxicity Tests The Report and Recommendations of an ECVAM/ ZEBET Workshop Altern Lab Anim. 2006;34:527-38. 
323 Green ML, Singh AV, Zhang Y, Nemeth KA, Sulik KK, Knudsen TB. Reprogramming of genetic networks during initiation of the Fetal Alcohol Syndrome. Dev Dyn. 2007;236:613-31.

324 Knudsen TB, Daston GP. Virtual tissues and developmental systems biology. In: Knudsen TB, Daston GP, editors. Comprehensive Toxicology: Developmental toxicology. New York: Elsevier; 2010. p. 347-58.

325 EPA. http://www.epa.gov/ncct/v-Embryo. Last accessed: Dec 2010.

326 Peters AK, Steemans M, Mesens N, Hansen E, Verheyen GR, Spanhaak S, et al. A higher throughput method to the Embryonic Stem cell Test (EST), to detect embryotocivity in early development. Altern Lab Anim. 2008;14:673-7.

327 Van Hummelen P, Sasaki J. State-of-the-art genomics approaches in toxicology. Mutat Res. 2010; 705:165-71.

328 Daston GP. The theoretical and empirical case for in vitro developmental toxicity screens, and potential applications. Teratology. 1996;53:339-44.

329 Toumadje A, Kusumoto K, Parton A, Mericko P, Dowell L, Ma G, et al. Pluripotent differentiation in vitro of murine ES-D3 embryonic stem cells. In vitro Cell Dev Biol Anim. 2003;39:449-53.

330 Robinson JF, Theunissen PT, van Dartel DAM, Pennings JLA, Faustman EM, Piersma AH. Examination of MeHg-induced toxicogenomic response across in vivo and in vitro models used for developmental toxicology. Submitted for publication. 2010.

331 Kistler A. Inhibition of chondrogenesis by retinoids: limb bud cell cultures as a test system to measure the teratogenic potential of compounds? Basel: Karger; 1985.

332 Dawson B, Trapp RG. Basic \& clinical biostatistics. Boston: Lange medical books; 2001.

333 Bogers R, De Vries-Buitenweg S, Geuijen I, van de Waart B, Kuiper R, Van Der Linden S, et al. An in vitro/in vivo screening assay as a sensitive tool to assess endocrine disruptive activity in surface water. Environ Int. 2007;33:292-301.

334 Leusch FD, de Jager C, Levi Y, Lim R, Puijker L, Sacher F, et al. Comparison of five in vitro bioassays to measure estrogenic activity in environmental waters. Environ Sci Technol. 2010;44:3853-60.

335 Pennings JLA, van Dartel DAM, Robinson JF, Pronk TE, Piersma AH. Gene set assembly for quantitative prediction of developmental toxicity in the Embryonic Stem cell Test. Toxicology. 2011; Accepted for publication.

336 zur Nieden NI, Kempka G, Ahr HJ. In vitro differentiation of embryonic stem cells into mineralized osteoblasts. Differentiation. 2003;71:18-27.

337 Schenk B, Weimer M, Bremer S, van der Burg B, Cortvrindt R, Freyberger A, et al. The ReProTect Feasibility Study, a novel comprehensive in vitro approach to detect reproductive toxicants. Reprod Toxicol. 2010;30:200-18. 

C

C

(0)

$0+(10)$

?

c

W

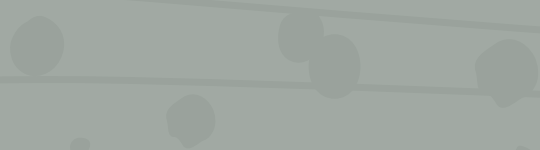

$2012-(1)$

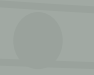

0

(

0

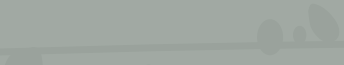

(7)

c

$0^{2}$

U

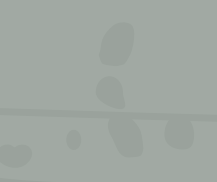

(2)

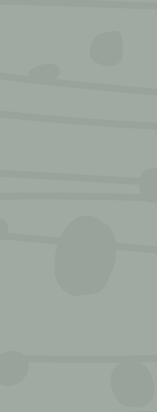

Q 0 
Dankwoord 

Nu mijn proefschrift afgerond is ben ik aan heel wat mensen mijn dank verschuldigd. Allereerst Aldert: Ik ben erg dankbaar voor de goede samenwerking de afgelopen jaren. Fijn dat ik altijd bij je binnen kon lopen en we mijn laatste bevindingen konden bespreken. Ik heb veel geleerd van jouw kritische en kundige blik op mijn resultaten. Zo'n overleg gaf me weer een flinke motivatie-boost en zorgde ervoor dat mijn onderzoek niet significant van de 'tijdstrack' afweek.

Jeroen, bedankt voor de inwijding in de wereld van de toxicogenomics. Je maakte altijd direct tijd om mijn vragen te beantwoorden en mijn manuscripten te bekritiseren. Ik vond het fijn dat ik met je samen mocht werken de afgelopen jaren.

Jos, ik ben blij dat je me de kans hebt gegeven dit onderzoek uit te mogen voeren. Ik ben Jos en Antoon ontzettend dankbaar dat ik mijn studies af heb kunnen ronden dankzij de verlenging van mijn aanstelling.

Frederik-Jan, als begeleider-op-afstand gedurende de eerste vier jaar van mijn project heb je altijd betrokkenheid bij mijn project getoond, bedankt!

Kirsten en Anja, mijn kamergenoten tijdens de start van mijn promotie onderzoek, bedankt dat jullie me wegwijs hebben gemaakt binnen GBO en op het lab. Aart en Peter, bedankt voor de gezellige tijd op de mannenvleugel van A3. Peter, dankjewel voor alle keren dat ik op je terug kon vallen in het lab. Ik ben blij dat je mijn paranimf wilt zijn!

Bert, Jolanda en Liset, dankzij jullie voorwerk kon ik een vliegende start maken, bedankt! Liset, een klein rekensommetje leert dat we samen ruim 1000 platen bekeken hebben, waarvan jij een groot deel voor jouw rekening genomen hebt, dank je wel!

Gelukkig heb ik ook de nodige hulp gehad van stagiaires: Sanne, Nicole en Sanna, heel erg bedankt voor jullie inzet.

Natuurlijk wil ik alle GBO collega's bedanken voor de waardevolle discussies bij de verschillende werkbesprekingen. Maar natuurlijk ook voor alle sociale en gezellige momenten, zoals de borrels, het befaamde voetbaltoernooi en de labuitjes.

I would like to thank my STW-project colleagues, as well as the STW task force 3 project members for your input and useful discussions.

Ik ben ook dank verschuldigd aan mijn Maastrichtse collega's, met name Joost, Marcel, Sandra en Karen. Hartelijk dank voor het runnen van mijn Affymetrix arrays, mede dankzij jullie heeft dat een prachtige dataset opgeleverd!

Loes, wat ben ik blij dat we 12 jaar geleden naast elkaar zijn gaan zitten met scheikunde! Van klasgenoot, studiegenoot, huisgenoot naar paranimf: Ik kan me haast geen idealere en efficiëntere paranimf wensen!

Pap en mam, fijn om te weten dat jullie trots op me zijn. Bedankt voor jullie interesse de afgelopen jaren en voor alle keren dat er weer een lekker verrassingsmaaltje klaar stond.

Lieve Harm, hoe druk en gestrest ik af en toe ook kon zijn, thuis was er rust. Ik ben je ontzettend dankbaar voor je vertrouwen en onvoorwaardelijke steun! Dit is het dan, mijn boekje. Het is af. 
C

C

(0)

$0+(10)$

?

c

W

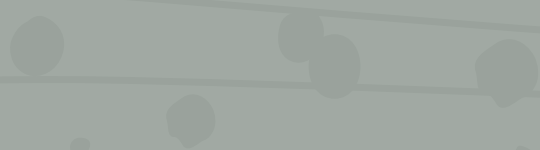

$2012-(1)$

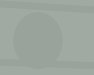

0

(

0

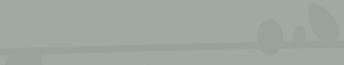

(7)

c

$0^{2}$

U

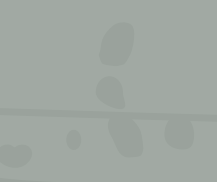

(2)

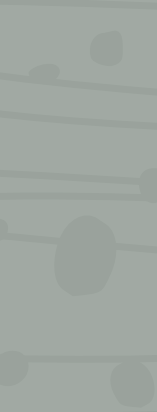

Q 0 
List of publications 

van Dartel DAM, Piersma AH. The Embryonic Stem cell Test combined with toxicogenomics as an alternative testing model for the assessment of developmental toxicity. Reprod Toxicol. 2011; Accepted for publication.

Pennings JLA, van Dartel DAM, Robinson JF, Pronk TE, Piersma AH. Gene set assembly for quantitative prediction of developmental toxicity in the Embryonic Stem cell Test. Toxicology. 2011;284:63-71.

van Dartel DAM, Pennings JLA, Robinson JF, Kleinjans JCC, Piersma AH. Discriminating classes of developmental toxicants using gene expression profiling in the Embryonic Stem cell Test. Toxicol Lett. 2011;201:143-151.

van Dartel DAM, Pennings JLA, de la Fonteyne LJJ, Brauers KJJ, Claessen SMH, van Delft $\mathrm{JH}$, Kleinjans JC, Piersma AH. Concentration-dependent gene expression responses to flusilazole in embryonic stem cell differentiation cultures. Toxicol Appl Pharmacol. 2011;251:110-118.

van Dartel DAM, Pennings JLA, de la Fonteyne LJJ, Brauers KJJ, Claessen SMH, van Delft $\mathrm{JH}$, Kleinjans JC, Piersma AH. Evaluation of developmental toxicant identification using gene expression profiling in embryonic stem cell differentiation cultures Toxicol Sci. 2011;119:126-134.

Pennings JLA, van Dartel DAM, Pronk TE, Hendriksen PJ, Piersma AH. Identification by gene co-regulation mapping of novel genes involved in embryonic stem cell differentiation. Stem Cells Dev. 2011; 20:115-126.

Piersma AH, van Dartel DAM, van der Ven LTM. Alternative Methods in Developmental Toxicology. In: Knudsen TB, Daston GP, editors. Comprehensive Toxicology: Developmental toxicology. New York: Elsevier; 2010;293-307.

Theunissen PT, Schulpen SH, van Dartel DAM, Hermsen SAB, van Schooten FJ, Piersma $\mathrm{AH}$. An abbreviated protocol for multilineage neural differentiation of murine embryonic stem cells and its perturbation by methyl mercury. Reprod Toxicol. 2010;29:383-92.

van Dartel DAM, Pennings JLA, de la Fonteyne LJJ, van Herwijnen M, van Delft JH, van Schooten FJ, Piersma AH. Monitoring developmental toxicity in the Embryonic Stem cell Test using differential gene expression of differentiation-related genes. Toxicol Sci. 2010;116:130-9. 
Osman AM, van Dartel DAM, Zwart E, Blokland M, Pennings JLA, Piersma AH. Proteome profiling of mouse embryonic stem cells to define markers for cell differentiation and embryotoxicity. Reprod Toxicol. 2010;30(2):322-32.

van Dartel DAM, Pennings JLA, van Schooten FJ, Piersma AH. Transcriptomics-based identification of developmental toxicants through their interference with cardiomyocyte differentiation of embryonic stem cells. Toxicol Appl Pharmacol. 2010;243:420-8.

van Dartel DAM, Zeijen NJL, de la Fonteyne LJJ, van Schooten FJ, Piersma AH. Disentangling cellular proliferation and differentiation in the Embryonic Stem cell Test, and its impact on the experimental protocol. Reprod Toxicol. 2009;28:254-61.

van Dartel DAM, Pennings JLA, Hendriksen PJ, van Schooten FJ, Piersma AH. Early gene expression changes during embryonic stem cell differentiation into cardiomyocytes and their modulation by monobutyl phthalate. Reprod Toxicol. 2009;27:93-102. 

C

C

(0)

$0+(10)$

?

c

W

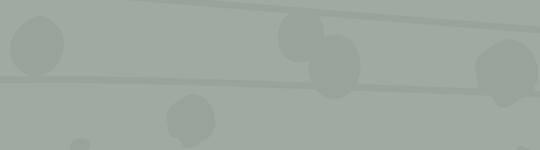

$2012-(1)$

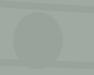

0

(

0

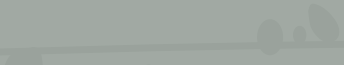

(7)

c

$0^{2}$

U

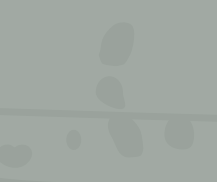

(2)

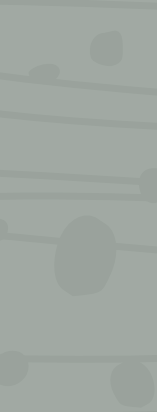

Q 0 
Curriculum Vitae 

Dorien Anna Maria van Dartel was born on February 31983 in Schijndel, the Netherlands. She graduated from secondary education at Gymnasium Beekvliet in Sint-Michielsgestel in 2001. In that same year, she started her study Biomedical Sciences at the Radboud University Nijmegen, with toxicology as a sub specialism. During her study she conducted her Master internship toxicology at the department of Toxicology and Drug Disposition at NV Organon under supervision of Josianne van Vliet and Jan-Peter Ploemen. Dorien obtained her Master's degree in 2006. In July 2006, she started as a PhD student at the department of Health Risk Analysis and Toxicology at Maastricht University. The research occurred within a cooperation with the laboratory for Health Protection Research at the National Institute for Public Health and the Environment (RIVM) where she was stationed. During this research period the Postgraduate Education in Toxicology was completed, which will result in the registration as toxicologist within the Netherlands Society of Toxicology. Since February 2011, Dorien has been working as a postdoctoral researcher at Human and Animal Physiology at Wageningen University. 
C

C

(0)

$0+(10)$

?

c

W

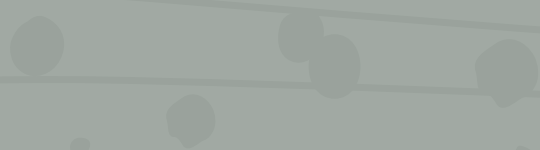

$2012-(1)$

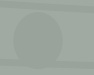

0

(

0

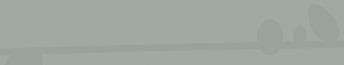

(7)

c

$0^{2}$

U

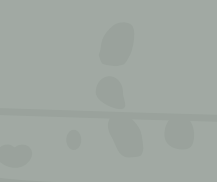

(2)

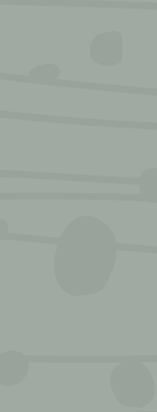

Q 0 
Color figures 


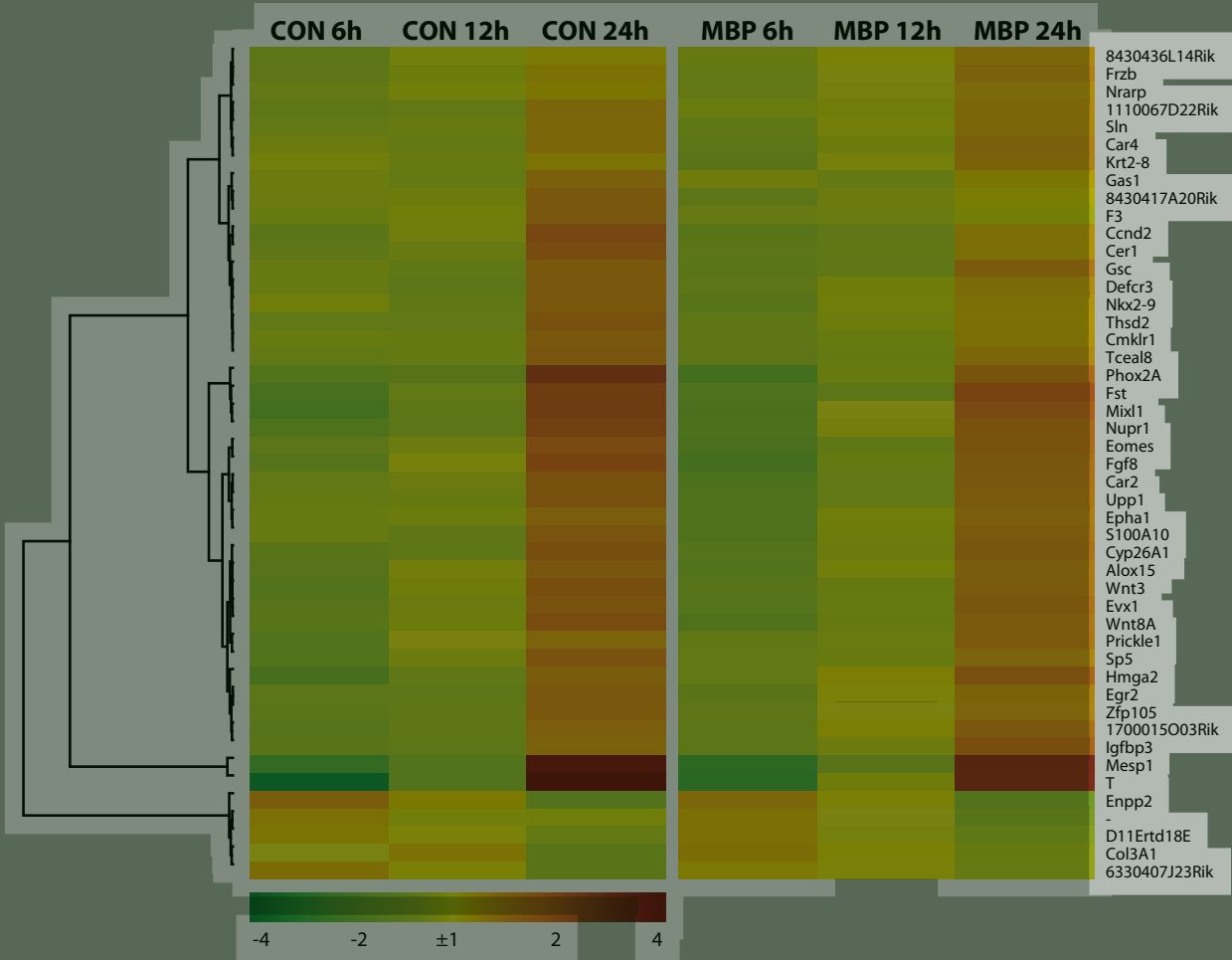

Chapter 3, Figure 3. Clustering of differentially expressed genes after $24 \mathrm{~h}$ of suspension culture. Cells were exposed for 6,12 or $24 \mathrm{~h}$ to monobutyl phthalate (MBP) or solvent control (CON). Per group the mean regulation of 7-8 replicates is shown. Gene expression changes are indicated by the color bar, where green represents down-regulation and red up-regulation. 
REGULATION_OF_PROGRESSION_THROUGH_CELL_CYCLE

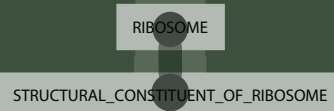

GLUTATHIONE_TRANSFERASE_ACTIVITY

RESPONSE_TO_UNFOLDED_PROTEIN

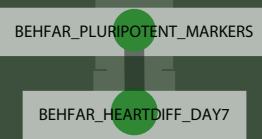

Cardiac gene set; upregulated

Pluripotency gene set; downregulated

Undefined gene set; upregulated

Undefined gene set; upregulated

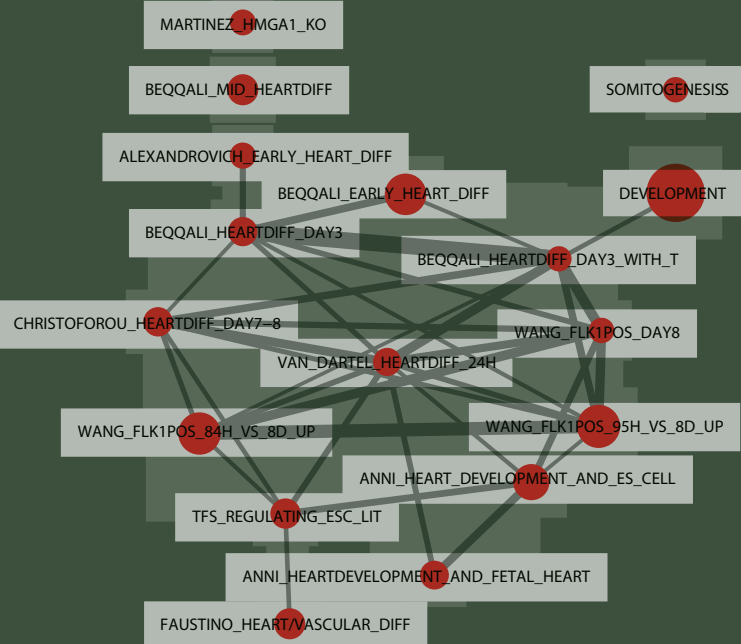

Chapter 3, Figure 4. Molecular concept analysis of regulated gene sets in differentiation. Per set the top $20 \%$ highest affected genes were used. The size of the gene set corresponds with the size of the node, and the boldness of the connecting lines indicates the degree of overlap between the respective gene sets.
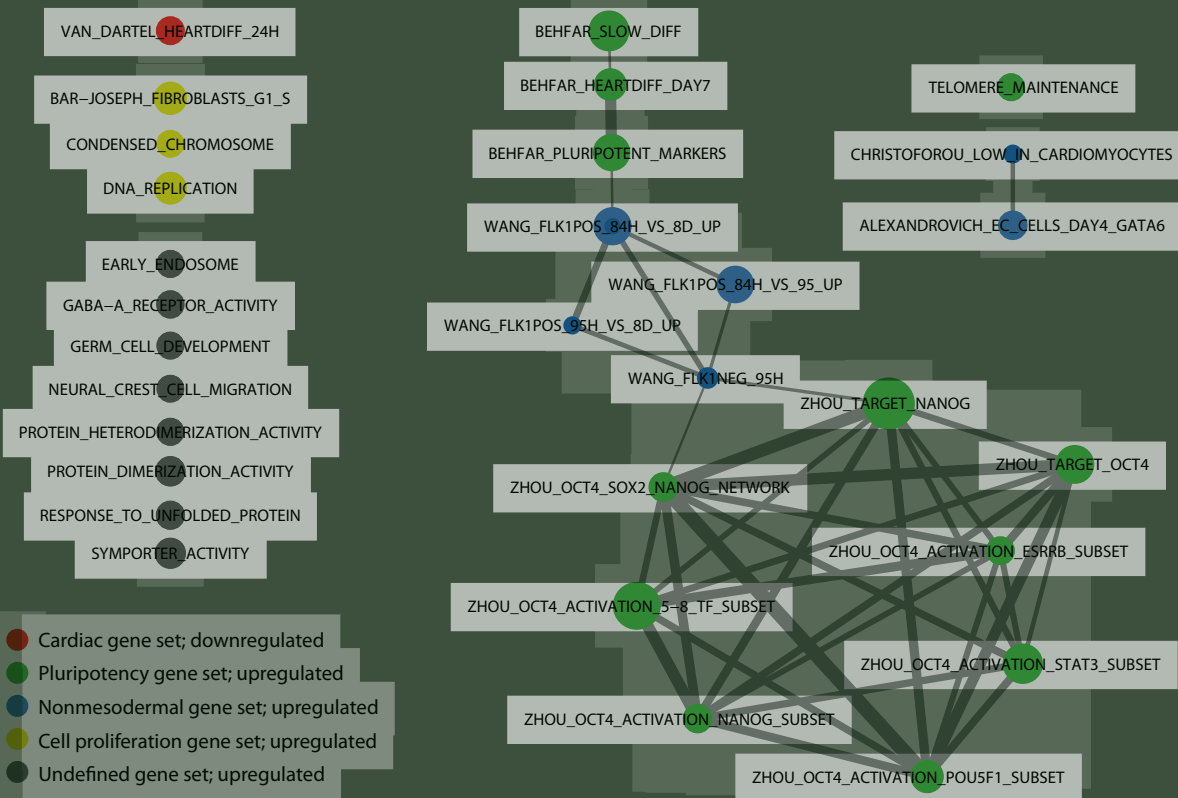

Chapter 3, Figure 5. Molecular concept analysis of regulated gene sets in monobutyl phthalate exposed differentiation cultures. Per set the top 20\% highest affected genes were used. The size of the gene set corresponds with the size of the node, and the boldness of the connecting lines indicates the degree of overlap between the respective gene sets. 

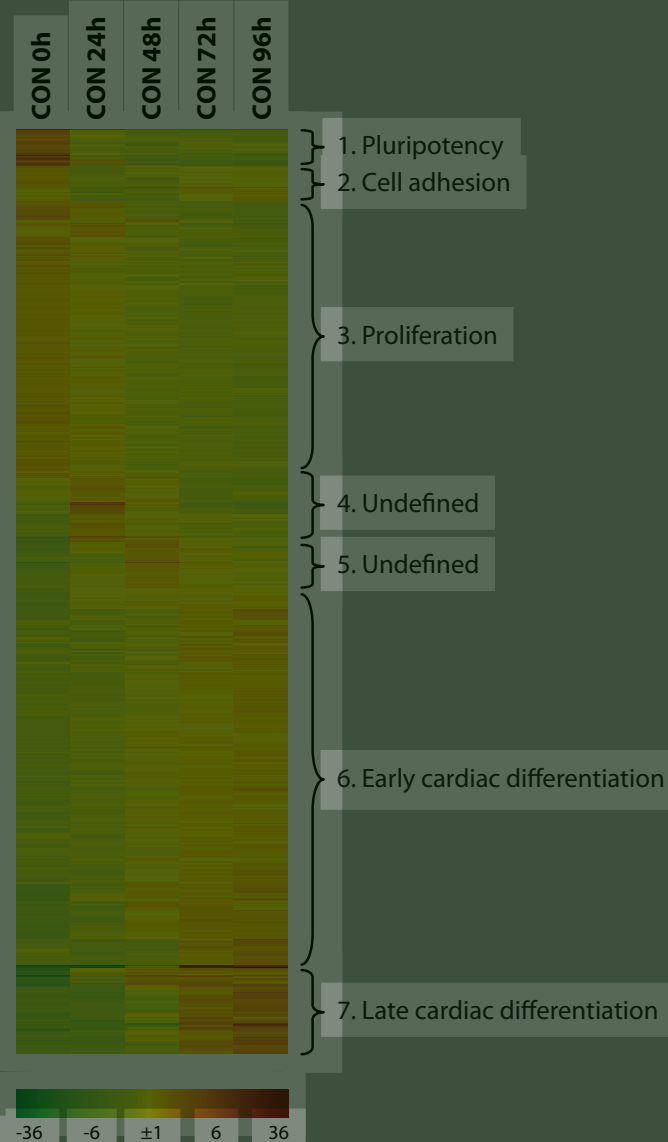

Chapter 4, Figure 2. Heatmap of all 1355 significantly regulated genes (fold ratio $>1.5$ and $p<0.001$ ) transcriptionally responsive to cardiac differentiation. Each row represents the relative expression of a single gene throughout the differentiation. Seven clusters are discriminated based on temporal gene expression of these regulated genes. The predominant function of each cluster is given. 


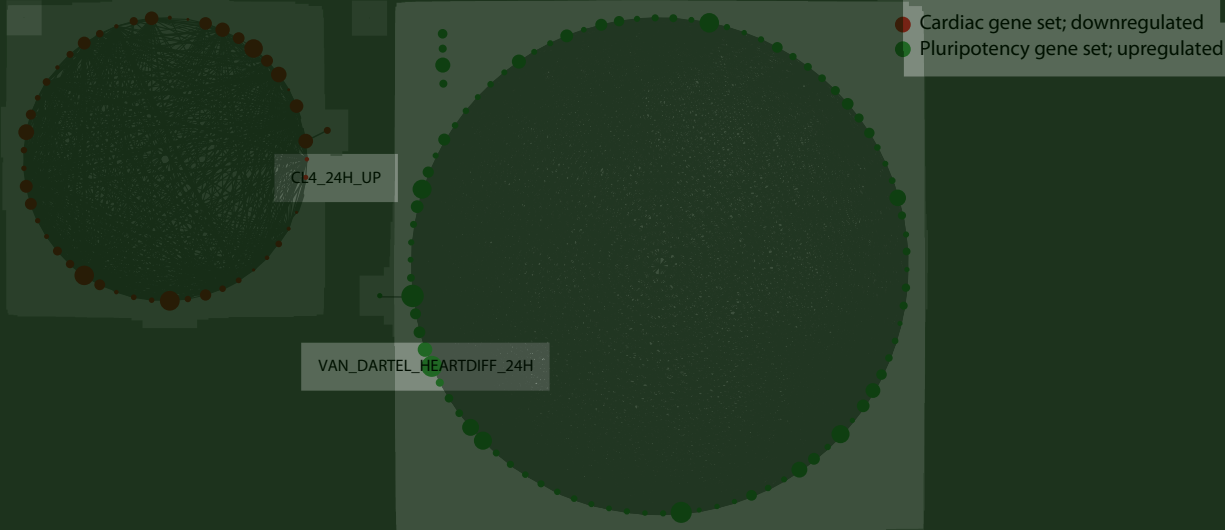

Chapter 4, Figure 3. Molecular concept analysis of regulated gene sets after $24 \mathrm{~h}$ of differentiation. Per set the top $20 \%$ highest affected genes were used. The size of the gene set corresponds with the size of the node, and the width of the connecting lines indicates the degree of overlap between the respective gene sets. 


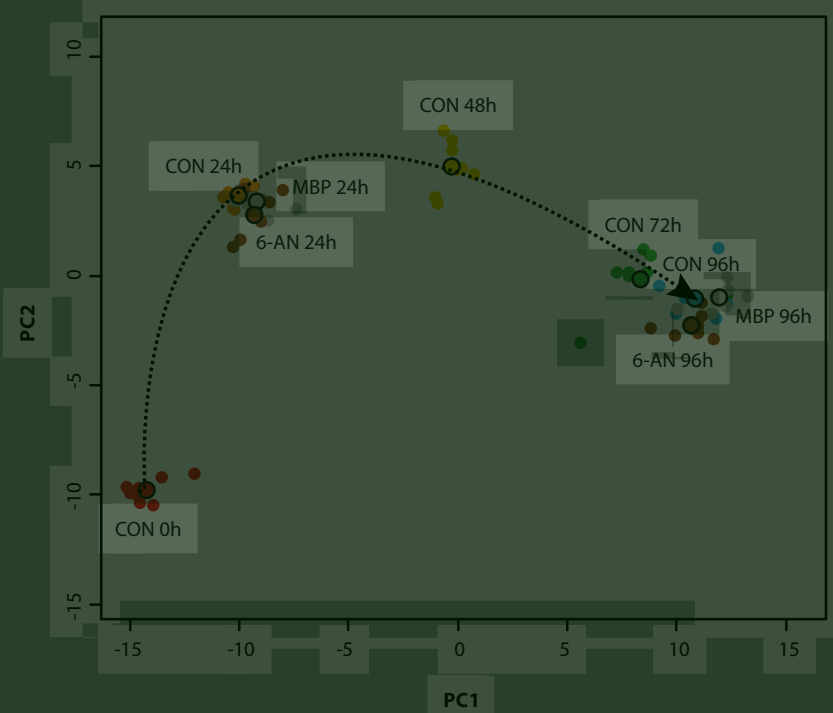

Chapter 4, Figure 4. Principal Component Analysis (PCA) of the control cultures after 0, 24, 48, 72 and $96 \mathrm{~h}$ of differentiation, and cultures exposed to monobutyl phthalate (MBP) or 6-aminonicotinamide (6-AN) for 24 or $96 \mathrm{~h}$. The PCA is based on the 1355 regulated genes. The mean values per experimental group are indicated by the large circles and the 'differentiation track' is shown by the dashed line.

A
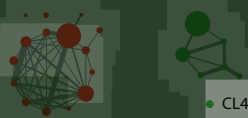

- $C L 4 \_24 \mathrm{H} \_$UP

Cardiac gene set; downregulated

Pluripotency gene set; upregulated
B

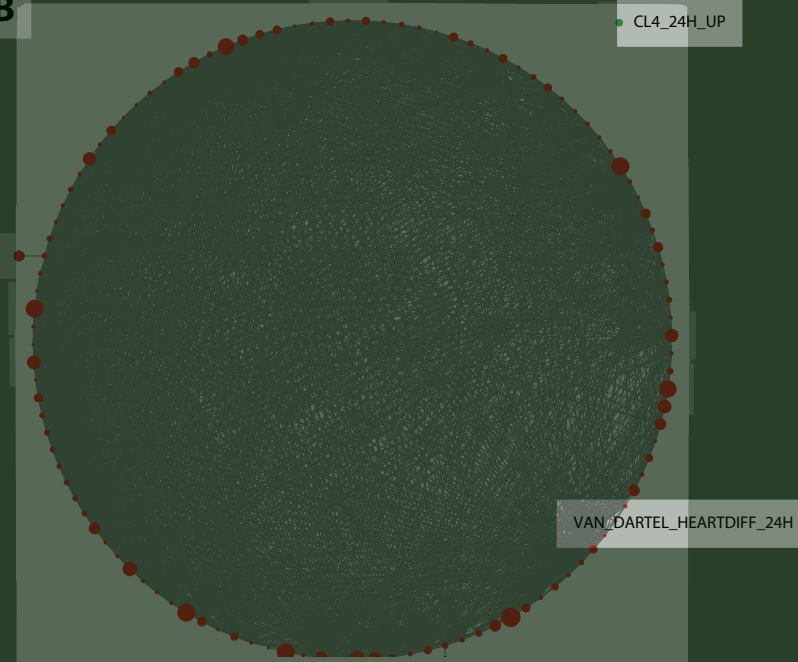

Chapter 4, Figure 5. Molecular concept analysis of regulated gene sets (A) after 24h monobutyl phthalate exposure in differentiation cultures and (B) after 24h 6-aminonicotinamide exposure in differentiation cultures. Per set the top $20 \%$ highest affected genes were used. The size of the gene set corresponds with the size of the node, and the width of the connecting lines indicates the degree of overlap between the respective gene sets. 


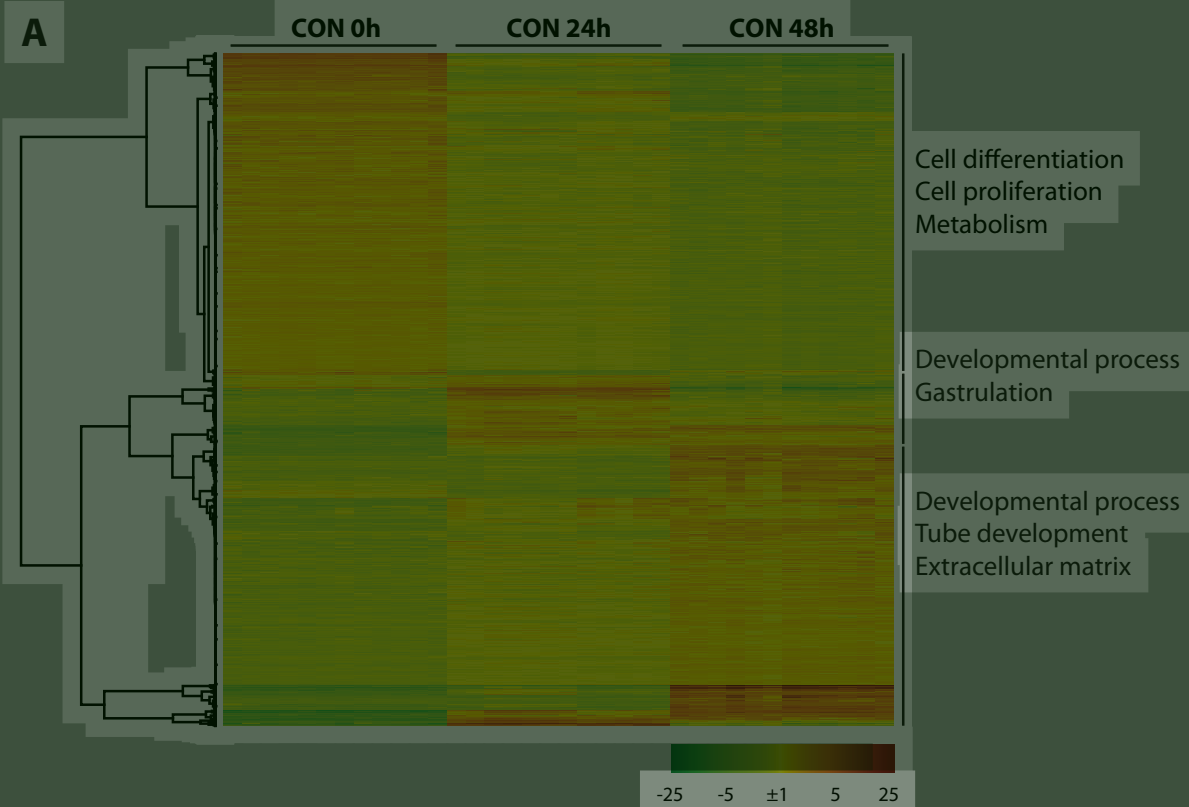

B

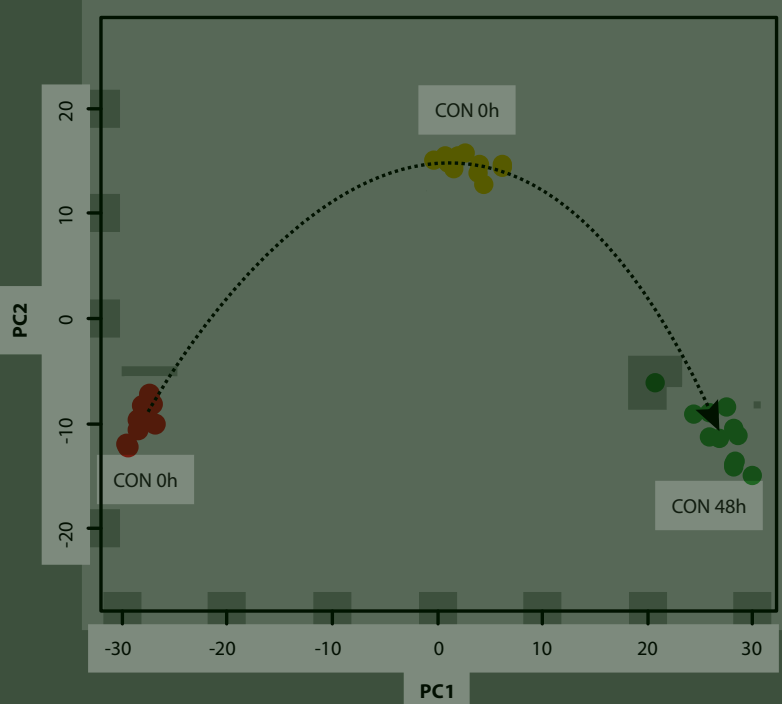

Chapter 5, Figure 3. (A) Clustering of the 3579 genes transcriptionally responsive to ESC differentiation between 0, 24 and $48 \mathrm{~h}$. Each row represents the relative expression of a single gene throughout differentiation. (B) Principal Component Analysis of unexposed control cultures (CON) after 0, 24 and 48h of differentiation using the 3579 differentiation-related genes. The arrow connecting these experimental groups represents the differentiation track. 


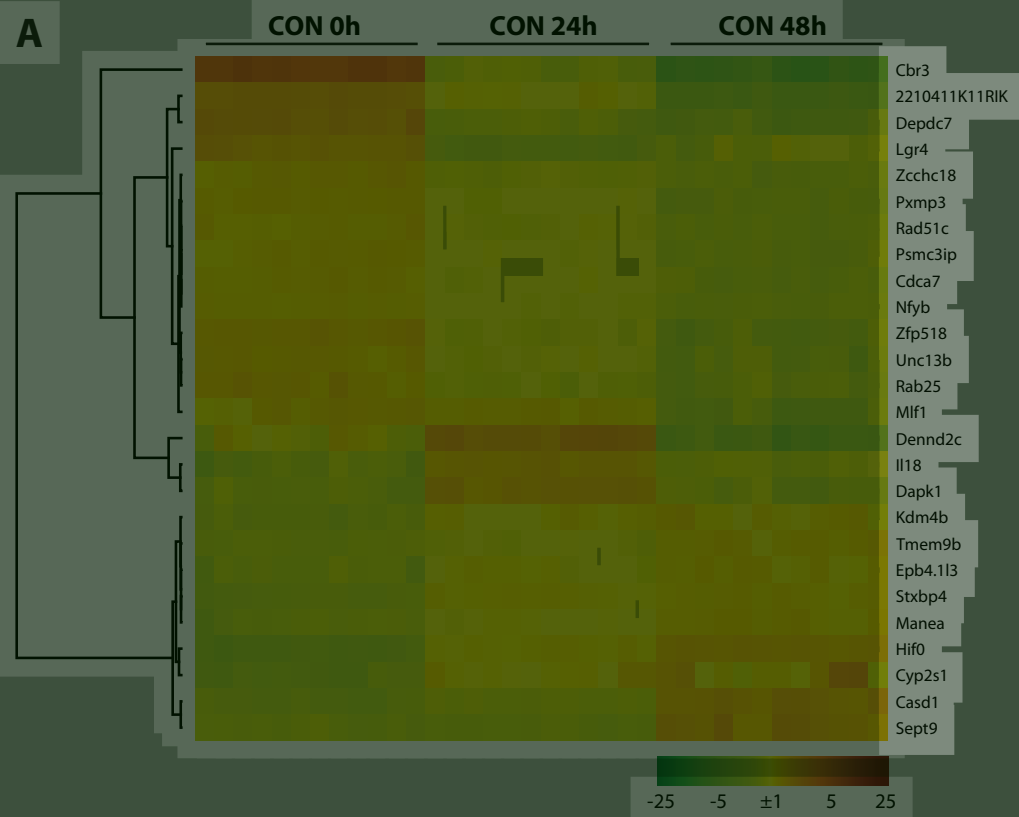

B

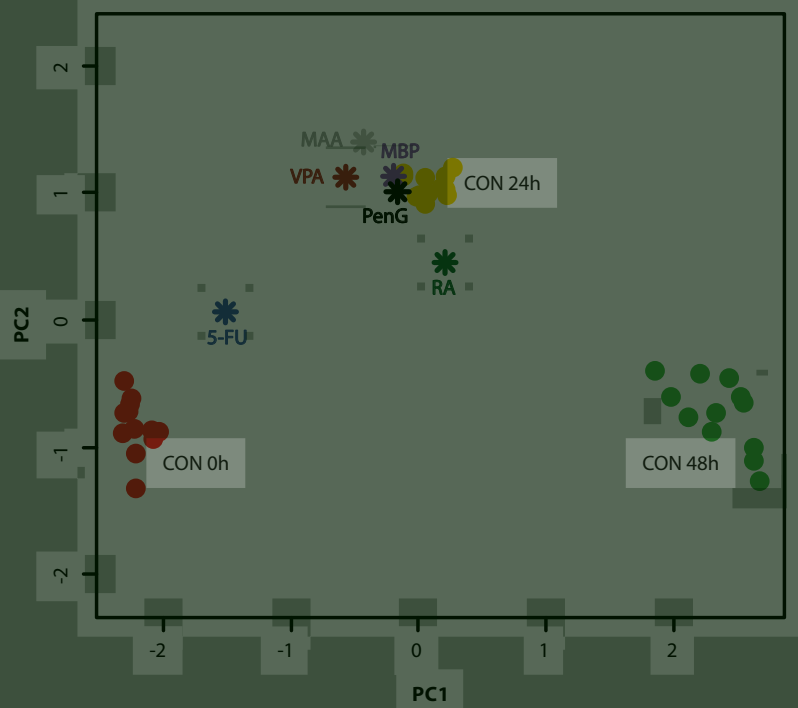

Chapter 5, Figure 4. (A) Clustering of the 26 genes that are both transcriptionally responsive to ESC differentiation with time and are also regulated by exposure to all developmental-toxic compounds tested. (B) Principal Component Analysis of the non-exposed cultures (CON) (individual samples, circles) at different stages of differentiation and compound-exposed cultures (mean of $n=8$ samples, asterisks) using the 26 biomarker genes. 


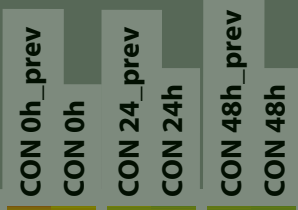

A
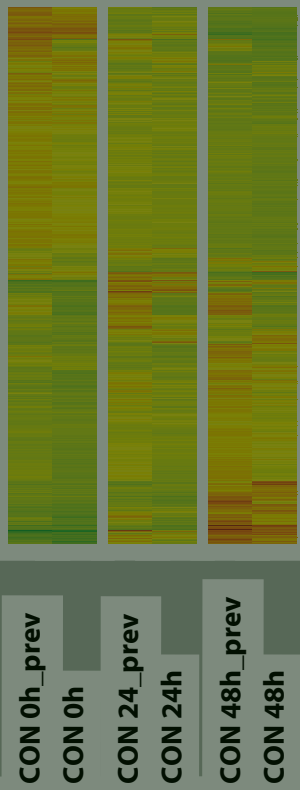

B

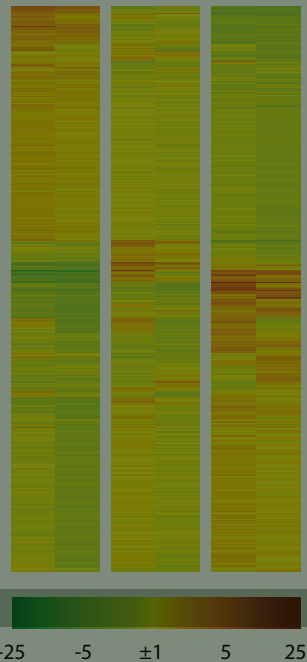

C

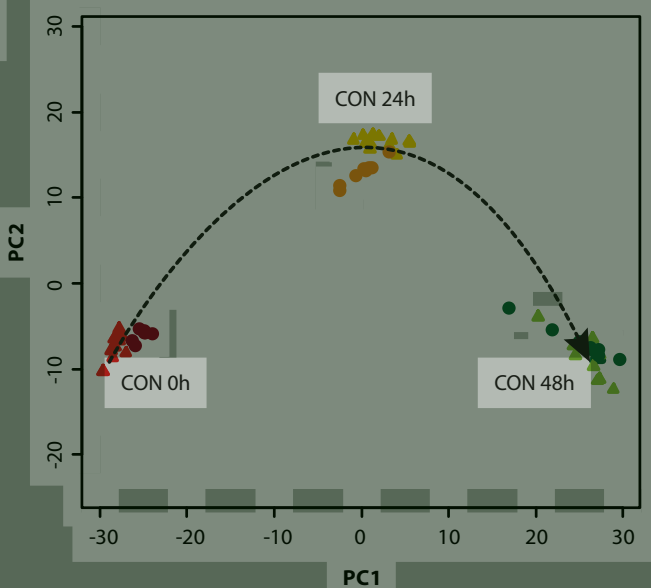

D

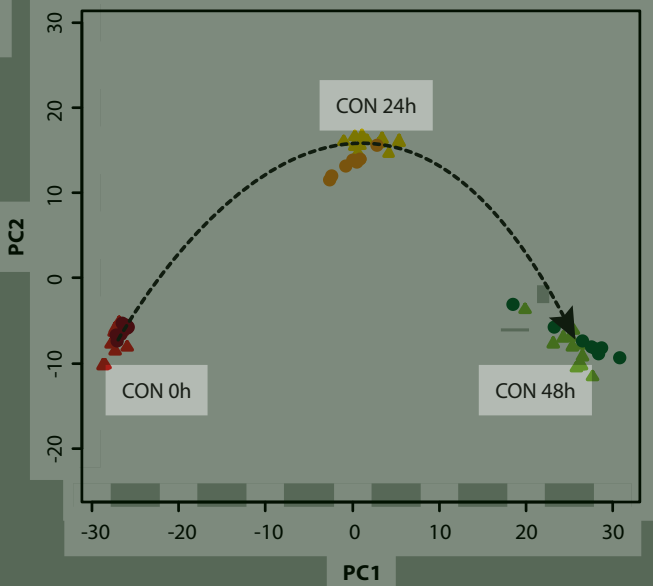

Chapter 6, Figure 1. Heatmap of the mean gene expression values of the unexposed control samples at 0,24 , and $48 \mathrm{~h}$ of differentiation in our previous (_prev; van Dartel et al., 2010a) and present study using the differentiated-related genes identified in our previous study (3579 genes; A) and in the present study (3780 genes, B). PCA plots based on ESC differention-related genes in our previous study (C) and the present study (D). Unexposed ESC differentiation samples of the previous study (circles) and the current study (triangles) are presented. 


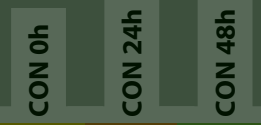

A
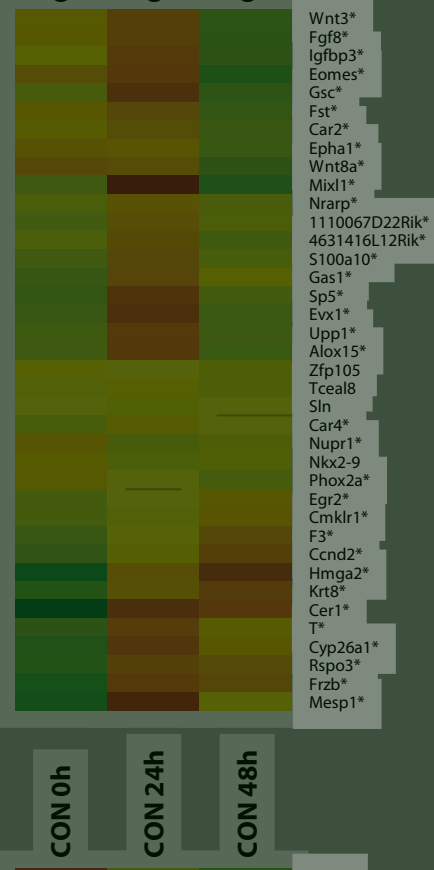

B

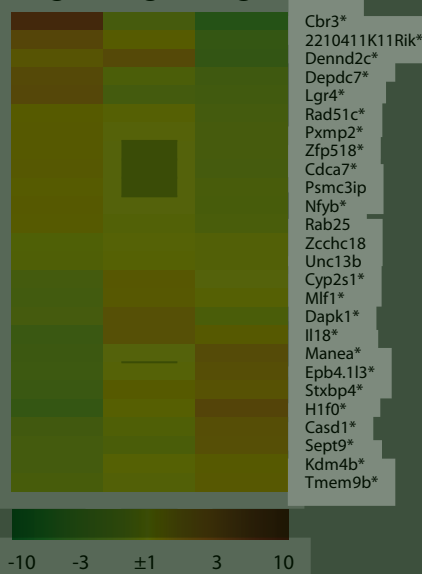

C

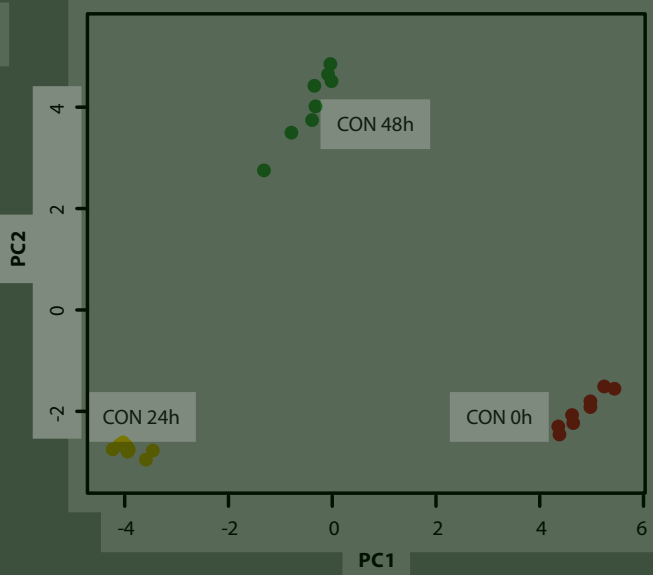

D

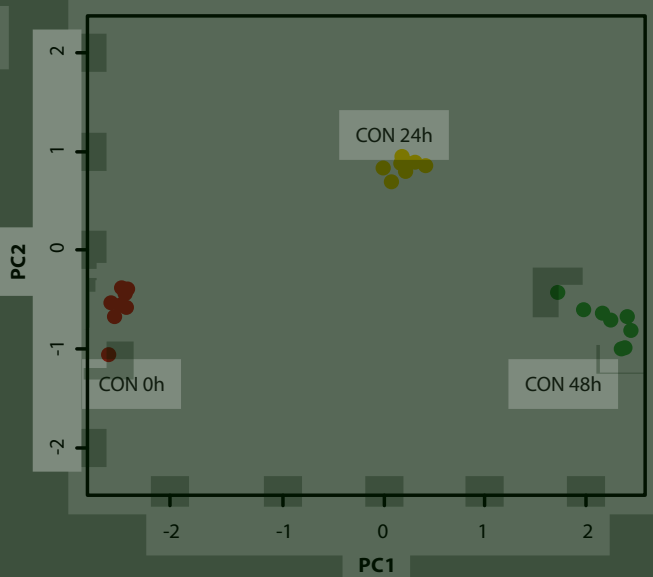

Chapter 6, Figure 3. Mean gene expression of the 'Van_Dartel_heartdiff_24h' (A) and 'EST biomarker genes' (B) gene sets at $0 \mathrm{~h}, 24 \mathrm{~h}$, and $48 \mathrm{~h}$ of control ESC differentiation cultures (CON). ${ }^{*}$ Indicate significantly differentially expressed genes among the $0 \mathrm{~h}, 24 \mathrm{~h}$, and $48 \mathrm{~h}$ CON groups. Principal Component Analysis of the control ESC differentiation cultures (individual samples, circles) at $0 \mathrm{~h}, 24 \mathrm{~h}$, and 48h of ESC differentiation using the 'Van_Dartel_heartdiff_24h' (C) and 'EST biomarker genes' (D). 
A

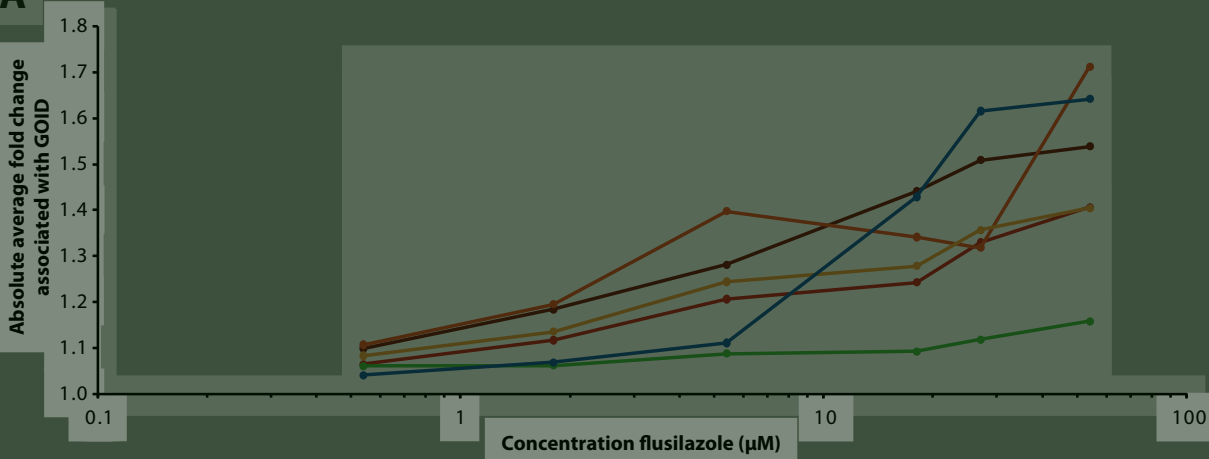

B

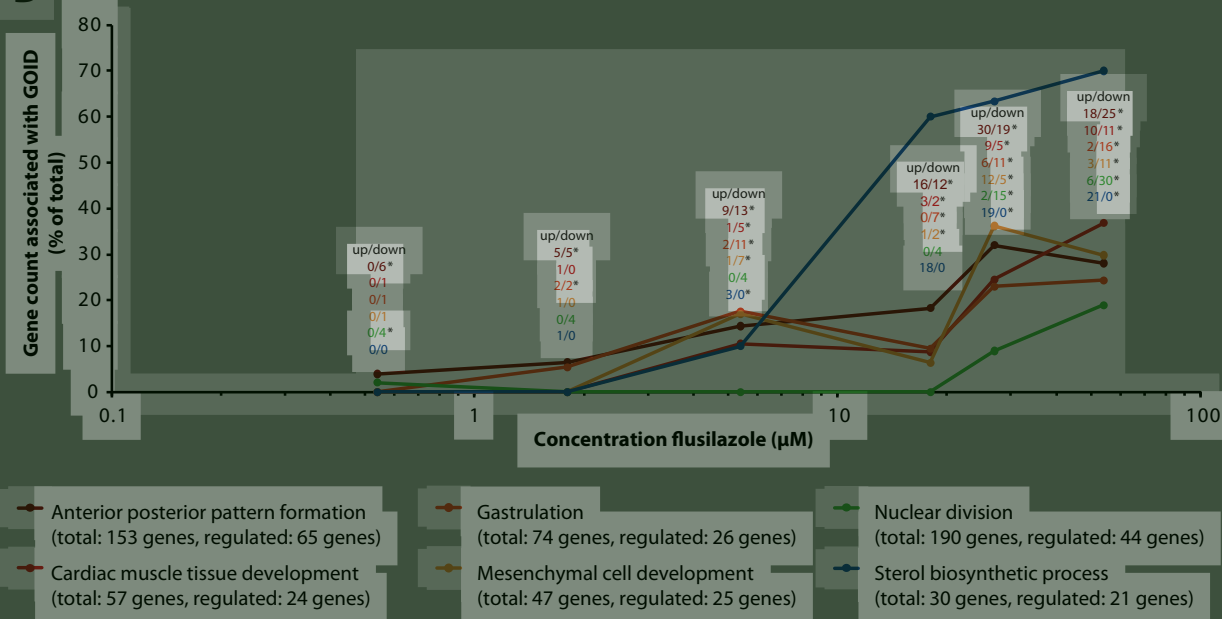

Chapter 7, Figure 2. (A) Concentration-related effect of flusilazole exposure in ESC differentiation cultures on absolute average fold change of a subset of enriched GOID. For comparability reasons the absolute average fold change was calculated using the significantly differentially expressed genes that contributed to the enrichment in at least one of the experimental groups. (B) Concentration-related effect of flusilazole exposure in ESC differentiation cultures on relative gene count associated with Gene Ontology Identifiers (GOID). GOID in red-yellow are related to development, in green to cell division and blue to lipid metabolism. 'Total' refers to total number of genes annotated to that GOID, and 'regulated' refers to total number of statistically significant genes that contributed to the enrichment of that GOID in at least one experimental group. Asterisks indicate statistically significantly enriched GOID. 

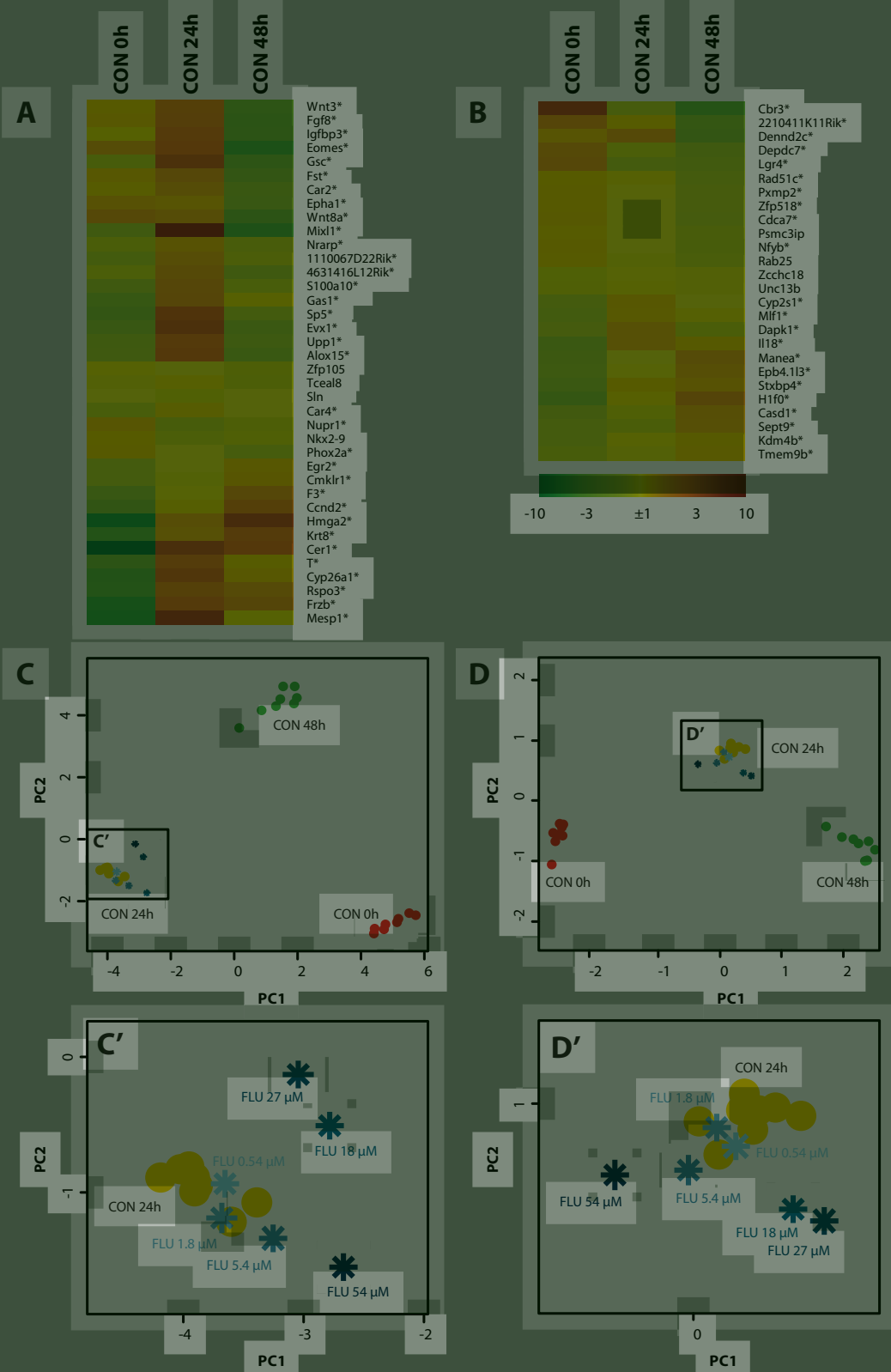

Chapter 7, Figure 4. Mean gene expression of the 'Van_Dartel_heartdiff_24h' (A) and 'EST biomarker genes' (B) gene sets at $0 \mathrm{~h}, 24 \mathrm{~h}$, and $48 \mathrm{~h}$ of control ESC differentiation cultures (CON). * Indicate significantly differentially expressed genes among the $0 \mathrm{~h}, 24 \mathrm{~h}$, and $48 \mathrm{~h}$ CON groups. Principal Component Analysis of the control ESC differentiation cultures (individual samples, circles) at $0 \mathrm{~h}, 24 \mathrm{~h}$, and $48 \mathrm{~h}$ of ESC differentiation and flusilazole-exposed cultures (mean of $n=8$ samples, asterisks) using the 'Van_Dartel_heartdiff_24h' (C) and 'EST biomarker genes' (D). C' and D' present the enlargement of the CON $24 \mathrm{~h}$ samples and the flusilazole-exposed cultures (FLU). 
A

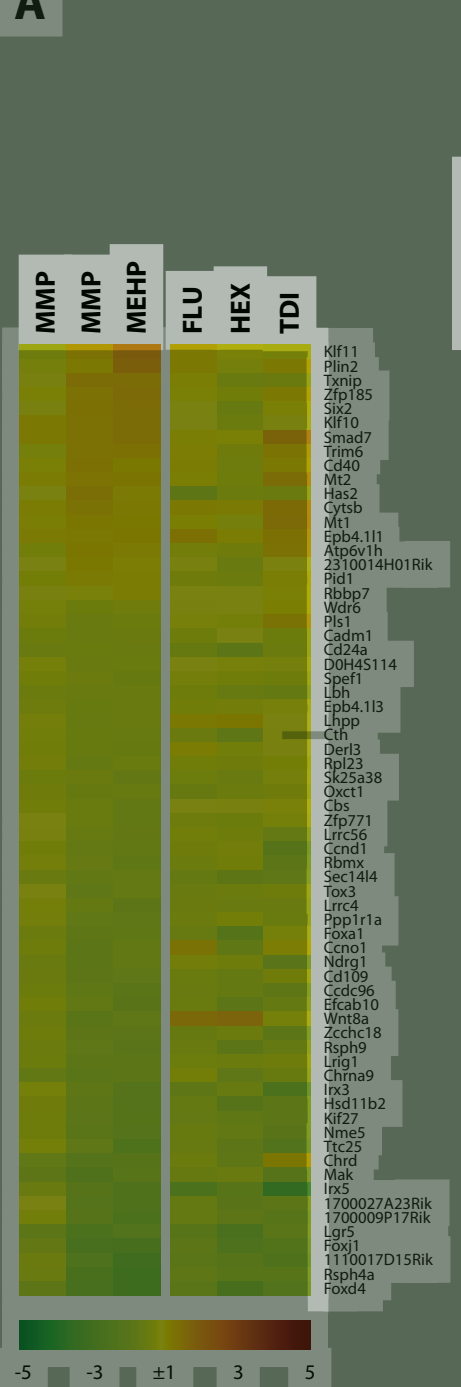

B

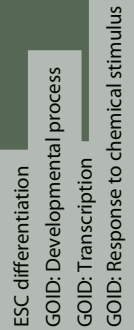

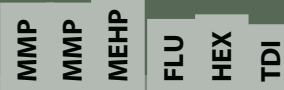

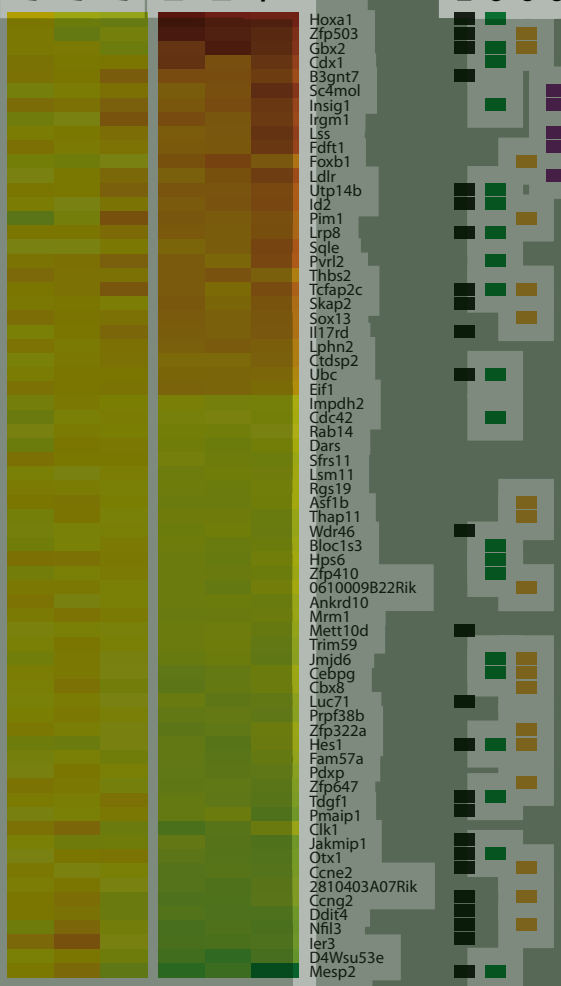

Chapter 8, Figure 3. Heatmap of the identified phthalate signature (A) and triazole signature (B). Responsiveness of the genes to early ESC differentiation and annotation of the genes to parent terms covering enriched GOID are indicated on the right of each figure. 

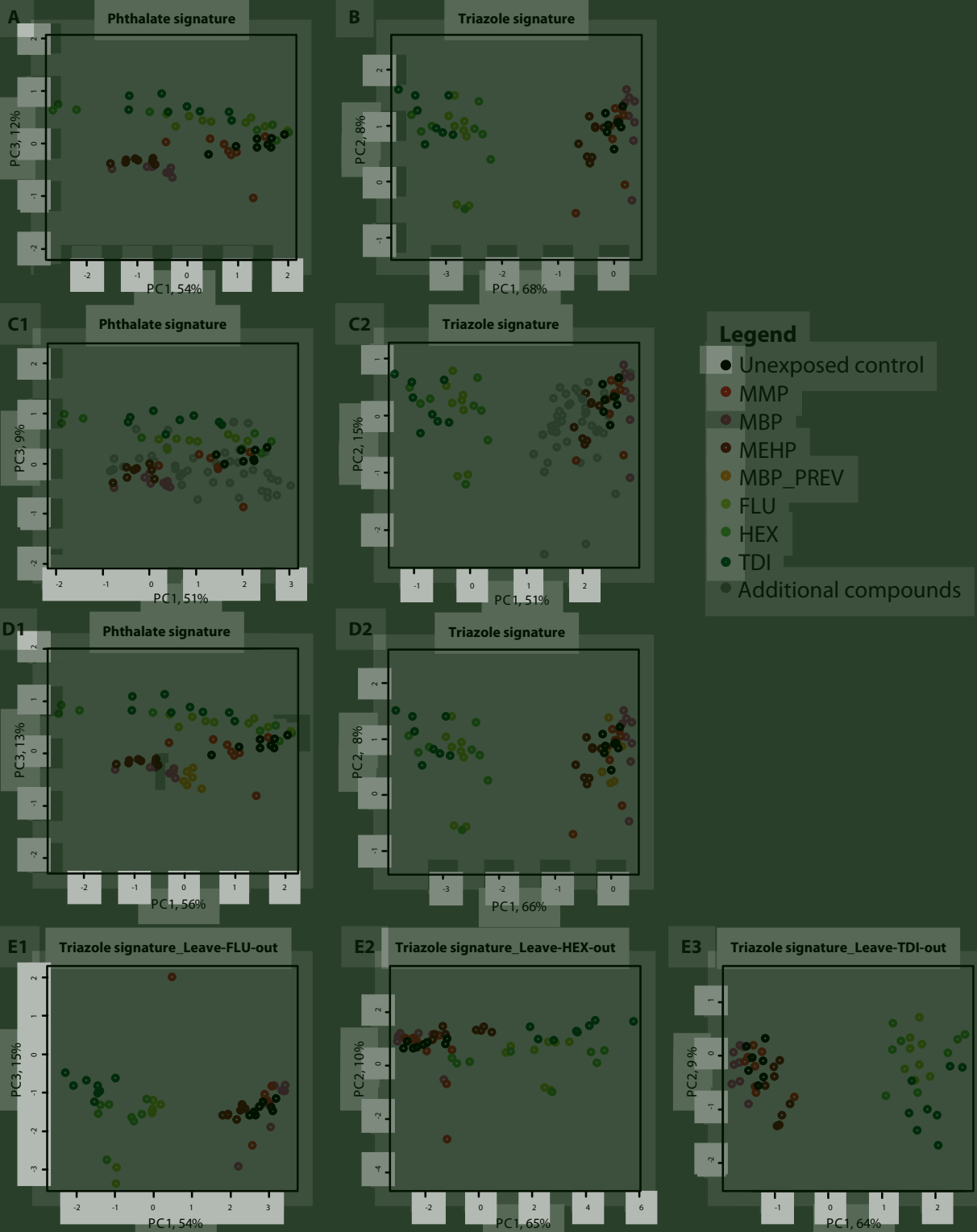

Chapter 8, Figure 4. Discrimination of phthalates from triazoles using principal component plots of monomethyl phthalate (MMP), monobutyl phthalate (MBP), monoethylhexyl phthalate (MEHP), flusilazole (FLU), hexaconazole (HEX) and triadimefon (TDI) on the basis of the identified phthalate signature (A) and the triazole signature (B). The principal component analysis-based discrimination was validated by studying the projection of additional compounds (C), by evaluating the position of MBP tested in our previous study (MBP_PREV, D), and by performing leave-one-out analyses (E). 
A. GOID enriched in phthalate signature

'Negative regulation cell differentiation' (222 genes)

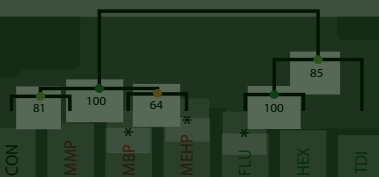

B. GOID enriched in triazole signature

'Anterior posterior pattern formation' (161 genes)

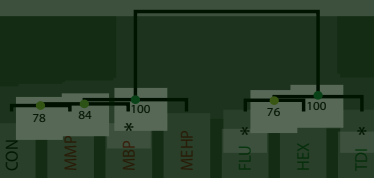

C. Predefined gene sets

'Van_Dartel_heartdiff_24h' (38 genes)

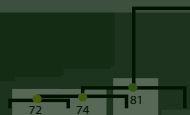

己 $\sum_{\Sigma}^{\infty} \sum^{\infty} \quad \frac{0}{\dot{1}}$

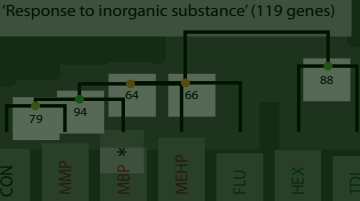

'Inner ear morphogenesis' (66 genes)

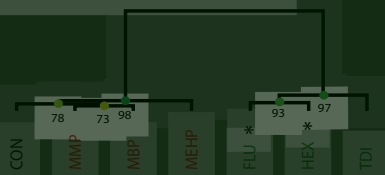

D. Negative control

'Genes not responsive to the test compounds' (200 genes)

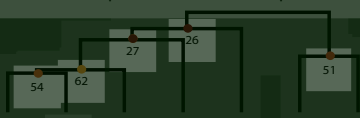

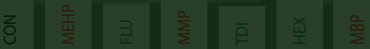

Color scale bootstrap value
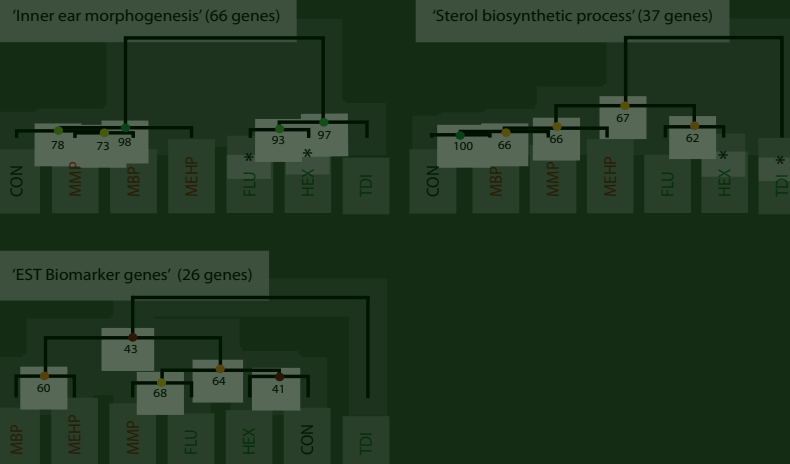

$\begin{array}{lllllll}00 & 90 & 80 & 70 & 60 & 50 & 40\end{array}$

Chapter 8, Figure 5. Hierarchical clustering of control (CON), monomethyl phthalate (MMP), monobutyl phthalate (MBP), monoethylhexyl phthalate (MEHP), flusilazole (FLU), hexaconazole (HEX) and triadimefon (TDI) using all genes annotated to gene ontology identifiers (GOID) enriched in the phthalate signature $(A)$ or triazole signature (B), using predefined gene sets (C), and using a negative control gene set (D). Asterisks indicate significant GOID enrichment ( $p$-value $<0.05$ ) within the significantly regulated genes ( $p$-value $<0.001$ ) of that compound. The color of the nodes indicates the confidence value (bootstrap value) for each node in the clustering tree. 
A

- Cell cycle

- Immune response

- Other

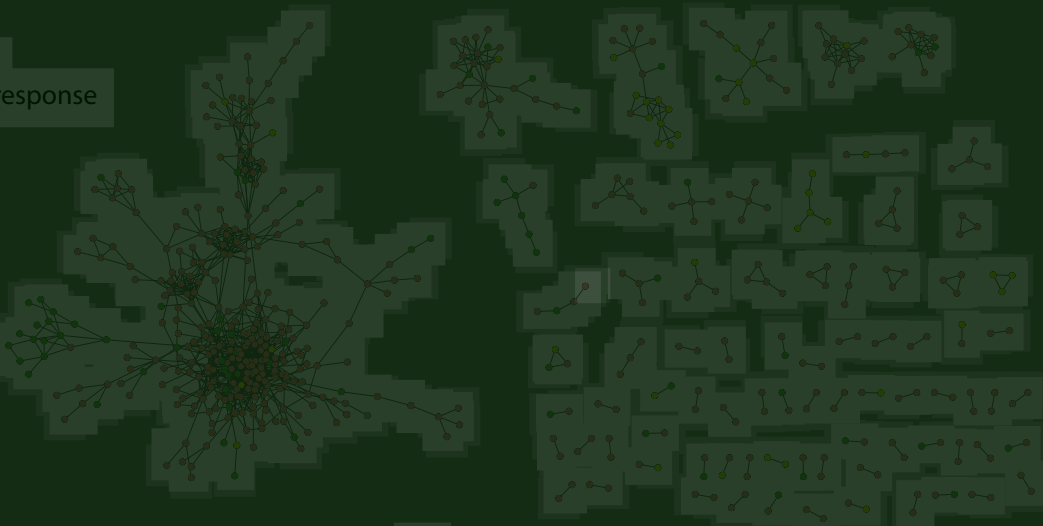

B

- Core inflammation response

Other immune response

- Other
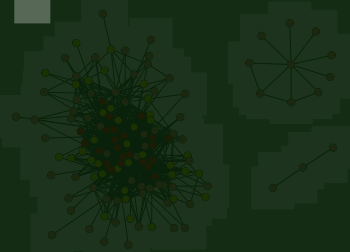

C

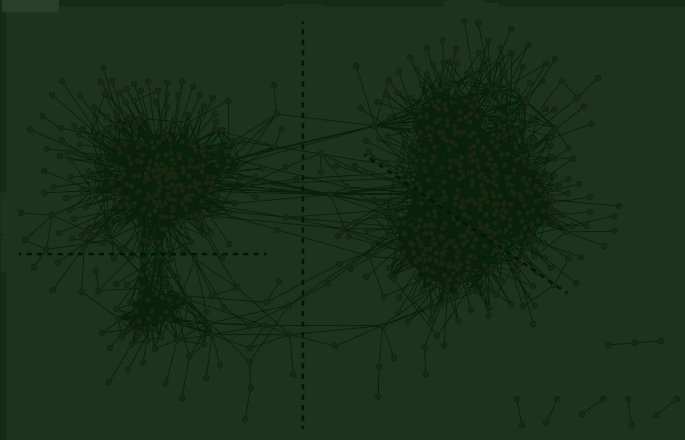

Chapter 10, Figure 1. Network visualizations for three gene set collections. A, network for c2.cgp collection (591 genes, 1243 edges); B, network for lung-inflammation collection (133 genes, 930 edges); C, network for ESC-diff (embryonic stem cell differentiation) collection (927 genes, 7402 edges) with transitions between sub-networks indicated as dashed lines. 
A

-/๑/O Novel

Stem cell development

/O Organ development

o - Genes in/outside other clusters

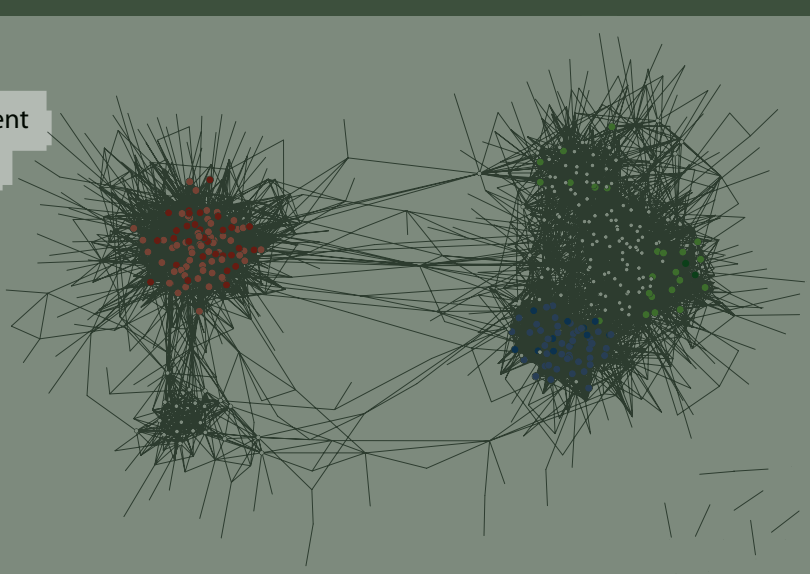

B

Early (day 3)

Middle (day 4-5)

Late (day 6-7)

Early and late

O No data

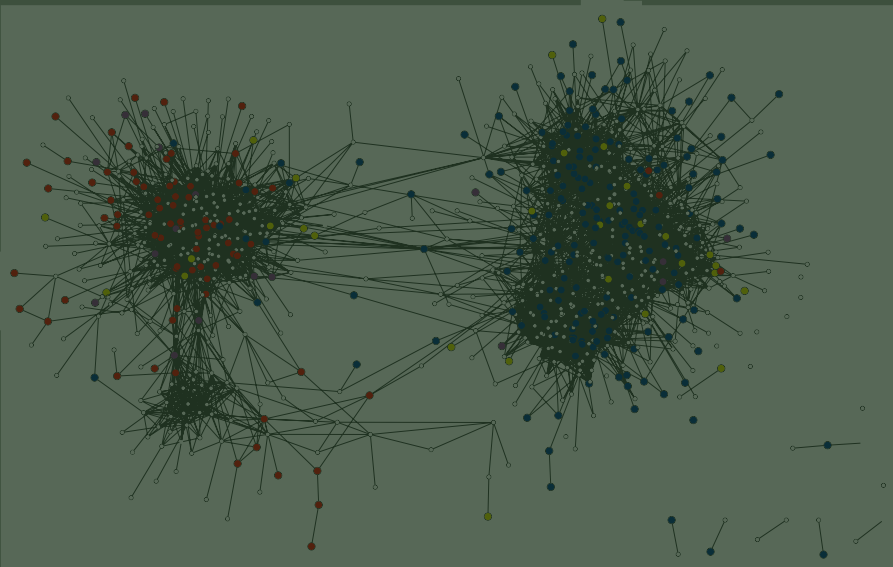

Chapter 10, Figure 2. Embryonic stem cell cardiomyocyte differentiation co-regulation network. A, biological process enrichment in network clusters containing novel functional candidates; $B$, peak gene expression for genes in a stem cell differentiation time series. 